\title{
Modulation of remyelination by adaptive inflammation and electrical stimulation
}

\author{
Dissertation \\ For the award of the degree \\ "Doctor rerum naturalium" (Dr. rer. nat.) \\ of the Georg-August-University Göttingen \\ within the doctoral program Molecular Medicine \\ of the Georg-August University School of Science (GAUSS)
}

submitted by

Patrik Kunz

born in

Dortmund, Germany

Göttingen, 2017 


\section{Members of the Thesis Committee}

\section{Supervisor}

Prof. Dr. Walter Paulus

Department of Clinical Neurophysiology

University Medical Center, Georg-August University Göttingen

Second member of the thesis committee

Prof. Dr. Wolfgang Brück

Department of Neuropathology

University Medical Center, Georg-August University Göttingen

\section{Third member of the thesis committee}

Prof. Dr. Jutta Gärtner

Department of Pediatrics

University Medical Center, Georg-August University Göttingen 


\section{Affidavit}

I hereby declare that my doctoral thesis entitled "Modulation of remyelination by adaptive inflammation and electrical stimulation" has been written independently with no other sources and aids than quoted.

Patrik Kunz

Göttingen, April 2017 
Meinen Eltern 


\section{List of publications}

\section{Original article}

Kunz P, Antal A, Hewitt M, Neef A, Opitz A, Paulus W (2017). 5 kHz Transcranial Alternating Current Stimulation: Lack of Cortical Excitability Changes When Grouped in a Theta Burst Pattern. Frontiers in Human Neuroscience 10, 1-8

\section{Abstract}

Kunz P, Stadelmann-Nessler C, Paulus W.

tACS in a rat model of focal demyelination.

$6^{\text {th }}$ International Conference of Transcranial Brain Stimulation, September $7^{\text {th }}-10^{\text {th }} 2016$, Göttingen, Germany, poster session

Kunz $\mathbf{P}^{\star}$, Escher $A^{\star}$, Brück W, Nessler S, Barrantes-Freer A, Stadelmann-Nessler, C Influence of autoimmune inflammation on remyelination in cuprizone-induced demyelination. $12^{\text {th }}$ European on Glia Cells on Health and Disease, July $15^{\text {th }}-18^{\text {th }} 2015$, Bilbao, Spain, poster session

*=equal contribution

Kunz P, Antal, A, Paulus, W

Modulation of cortical excitability by grouped electrical stimulation protocols.

$30^{\text {th }}$ International Congress of Clinical Neurophysiology, March $19^{\text {th }}-23^{\text {rd }} 2014$, Berlin, Germany, poster session 


\section{Table of Content}

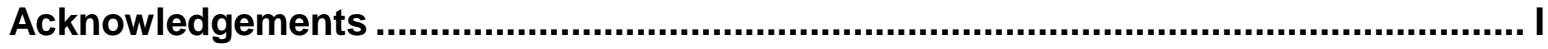

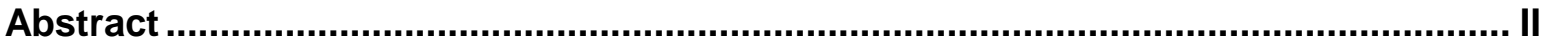

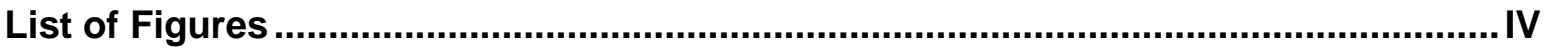

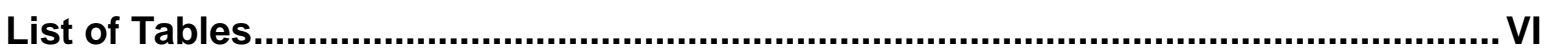

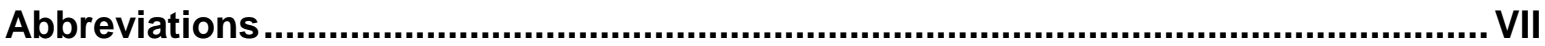

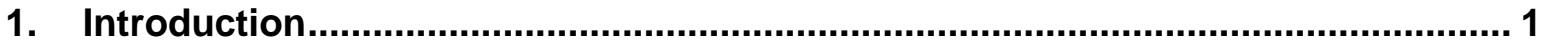

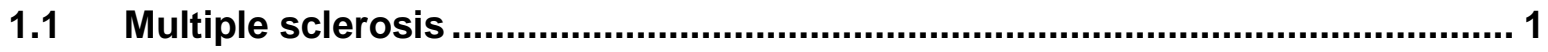

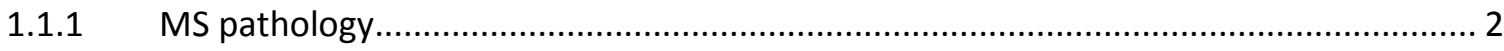

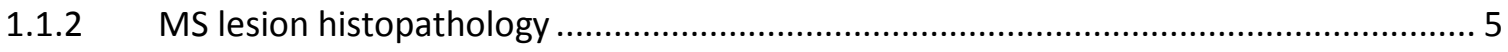

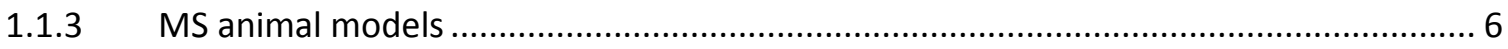

1.1.3.1 Experimental autoimmune encephalomyelitis ................................................... 6

1.1.3.2 Toxic demyelination by Cuprizone administration.................................................... 7

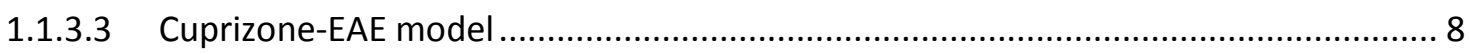

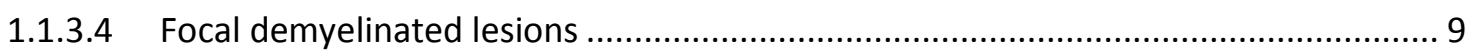

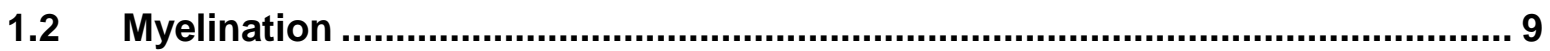

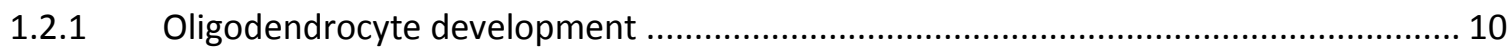

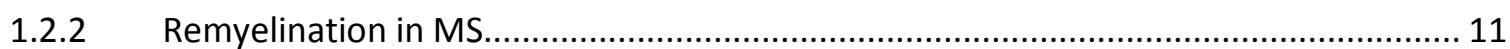

1.3 Electrical stimulation in neurodegenerative diseases.................................13

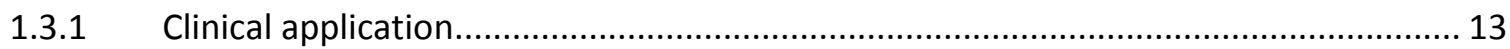

1.3.2 Influence of electrical stimulation on neuronal progenitor cells ................................... 14

1.3.3 Peripheral nerve regeneration/remyelination by electrical stimulation ....................... 14

1.3.4 Influence of electrical stimulation on CNS remyelination .............................................. 15

1.4 Transcranial electrical stimulation................................................................18

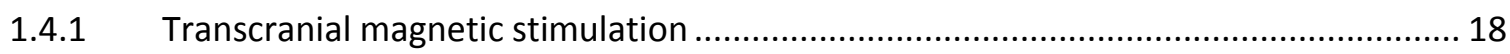

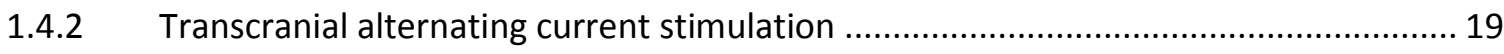

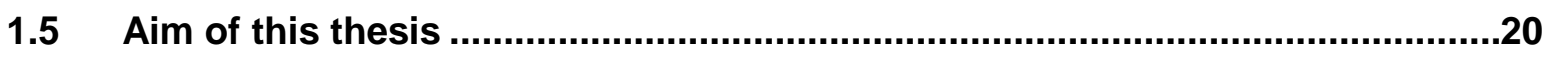

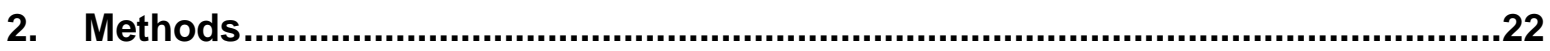

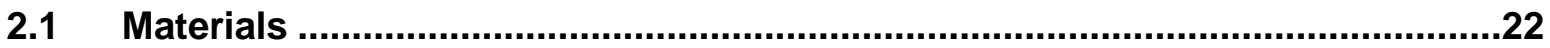

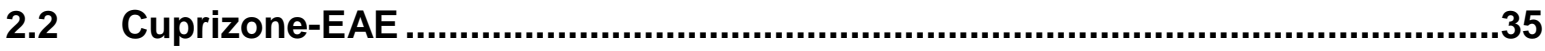

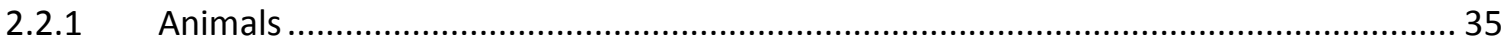




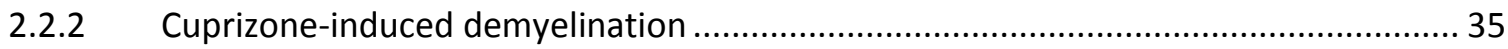

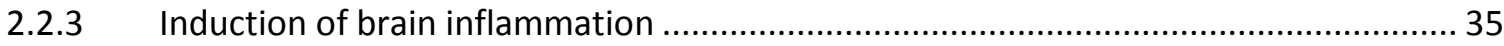

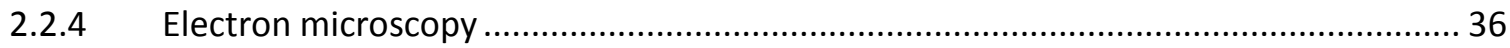

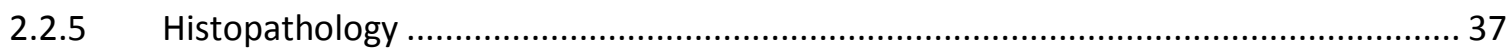

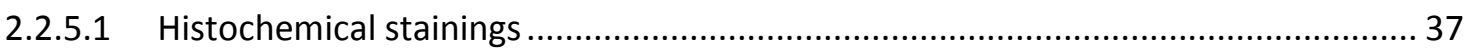

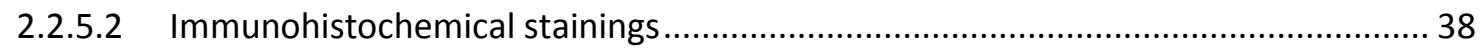

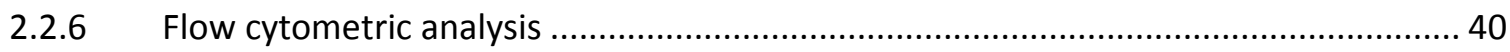

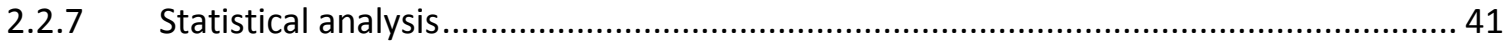

2.3 Electrical stimulation in mixed cortical cultures............................................43

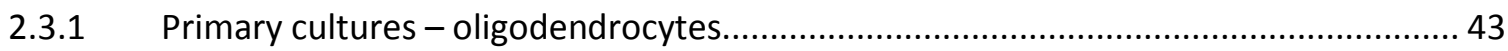

2.3.2 Primary cultures - oligodendrocytes and neurons ................................................... 44

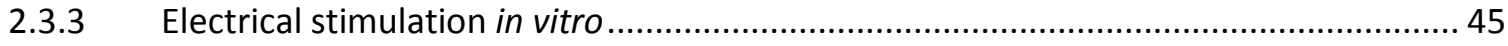

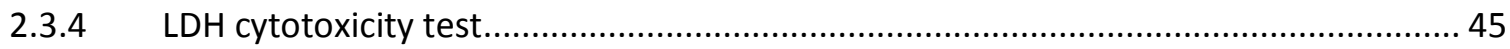

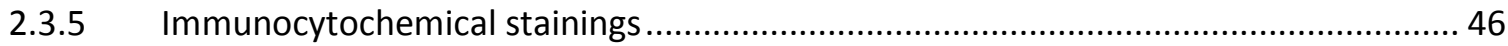

2.3.6 Quantification of myelin protein expression in oligodendrocyte cultures ................... 46

2.3.7 Quantification of myelinated fibers in oligodendrocyte/neuron mixed cultures ......... 46

\subsection{Electrical stimulation in rodent models of demyelination ................................47}

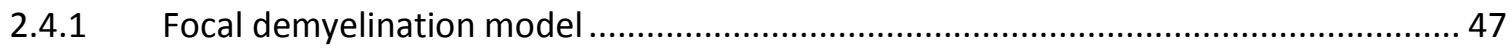

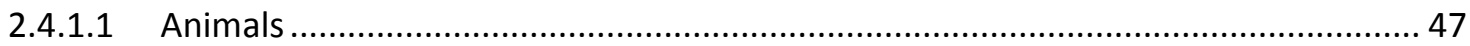

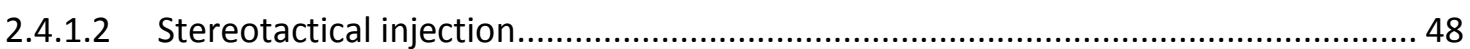

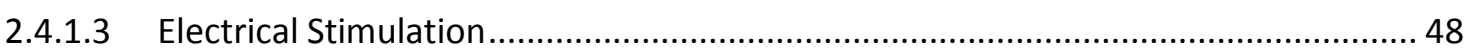

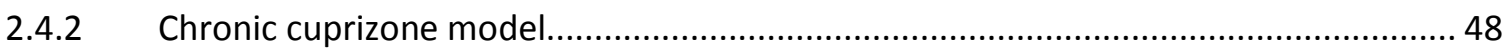

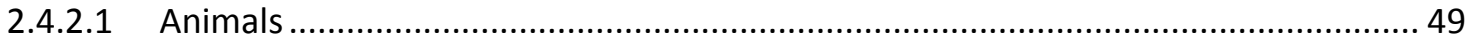

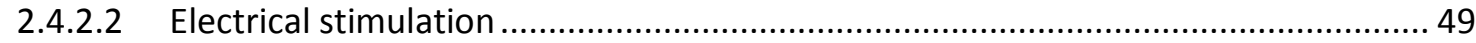

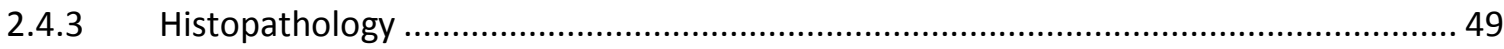

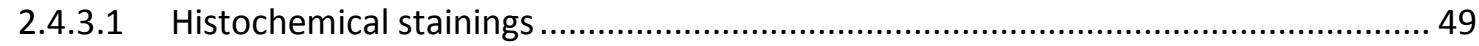

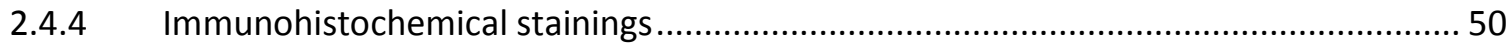

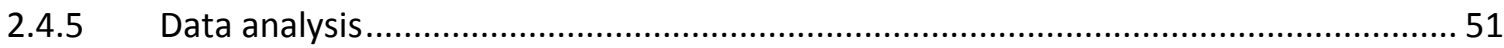

2.5 Transcranial electrical stimulation in healthy human subjects ......................54

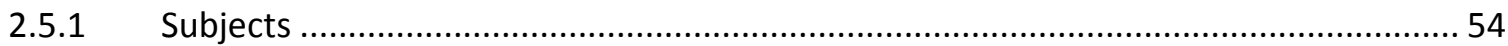

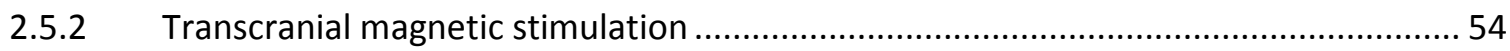

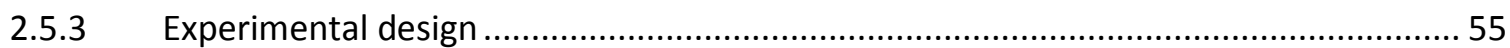

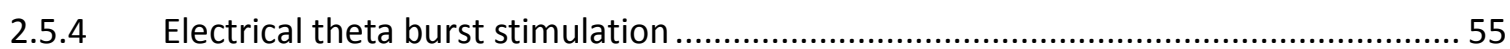

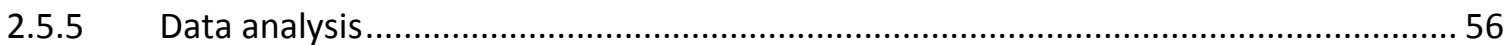


3.1 Influence of autoimmune inflammation on remyelination in Cuprizone-induced demyelination

3.1.1 Cuprizone administration for 4.5 weeks leads to demyelination in the corpus callosum in Cup and Cup-EAE animals....

3.1.2 Increased infiltration and activation of immune competent cells in Cuprizone/MOG treated animals.

3.1.3 The number of OPC and mature OLG is largely unaffected by EAE induction

3.1.4 The density of myelin producing cells and degree of remyelination remain unchanged after immunization with $\mathrm{MOG}_{35-55}$ peptide.

3.1.5 Increased axonal damage in Cuprizone/MOG treated animals

3.1.6 The density of myelinated axons is unaffected by EAE induction.

\subsection{Influence of transcranial alternating current stimulation on spontaneous} remyelination in in vitro and in vivo models

3.2.1 Influence of alternating current stimulation on cell viability and myelin production in primary oligodendroglial cell cultures and mixed cortical cell cultures.

3.2.1.1 The amount of myelin producing oligodendrocytes in primary oligodendrocyte cultures remains unchanged after electrical stimulation

3.2.1.2 Myelination and cell viability in mixed cortical cell cultures are unaffected by electrical stimulation

3.2.2 Influence of transcranial alternating current stimulation on spontaneous remyelination in rodent models of demyelination

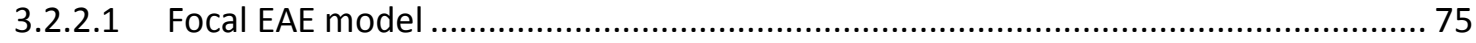

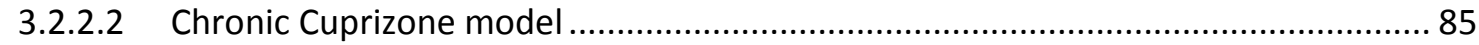

3.3 Transcranial electrical stimulation in healthy human subjects .......................92

3.3.1 MEP amplitudes after eTBS did not differ from MEP after sham treatment.

3.3.2 No significant effect on MEP amplitudes after stimulation with altered ecTBS protocols

4.1 Influence of autoimmune inflammation on remyelination in Cuprizone-induced demyelination

4.1.1 Increased infiltration of the CNS by immune competent cells in Cup-EAE mice 97

4.1.2 Damaging and neuroprotective effects of autoimmune inflammation.... 98

4.1.3 Influence of adaptive inflammation on oligodendrocyte populations and remyelination

4.2 Influence of transcranial alternating current stimulation on spontaneous remyelination in in vitro and in vivo models 
4.2.1.1 Safe application of $10 \mathrm{~mA}$ alternating currents................................................... 102

4.2.1.2 Electrical stimulation failed to increase myelination in vitro ................................. 102

4.2.2 Electrical stimulation in rodent models of demyelination ........................................ 103

4.2.2.1 Induction of focal demyelinated lesions after intracortical injection of anti-MOG antibody and complement ......................................................................... 103

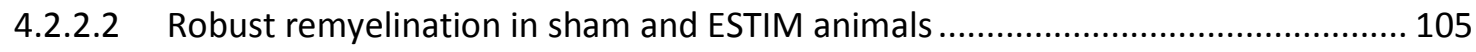

4.2.2.3 Heterogeneous remyelination of the corpus callosum after 13 weeks of Cuprizone administration ..... 107

4.2.2.4 ESTIM failed to exert effects on remyelination or oligodendrocyte populations... 108

4.3 Transcranial electrical stimulation in healthy human subjects .111

4.3.1 Differences in electric field strength and orientation may cause lack of effects on cortical excitability by eTBS protocols

4.3.2 Safe application of $10 \mathrm{~mA}$ eTBS but high interindividual variability 112

5. Summary and conclusion

5.1 Influence of autoimmune inflammation on remyelination in Cuprizone-induced demyelination

5.2 Influence of transcranial alternating current stimulation on spontaneous remyelination in in vitro and in vivo models

5.3 Transcranial electrical stimulation in healthy human subjects

6. Bibliography 


\section{Acknowledgements}

First and foremost, I would like to thank my supervisors Prof. Dr. Walter Paulus and Prof. Dr. Stadelmann-Nessler for their great and constant support, their guidance as well as for providing me with the opportunity to pursuit my own ideas. I am particularly thankful for all the interesting and multi-facet experiences I was able to gain by working in the Department of Clinical Neurophysiology as well as in the Department of Neuropathology. Furthermore, I am grateful for all the inspiring discussions, which frequently took place despite very tight schedules and I enjoyed the professional but also familiar atmosphere in both Departments.

I would also like to thank Prof. Dr. Wolfgang Brück, not only for giving me the opportunity to work in the Department of Neuropathology but also for being part of my thesis committee and for the inspiring discussions during the thesis committee meetings. In line with this, many thanks to Prof. Dr. Jutta Gärtner for being the third member of my thesis committee and for the helpful contributions during the meetings.

Moreover, I want to thank Dr. Stefan Nessler, Dr. Claudia Wrzos, Dr. Anne Winkler, Dr. Alonso Barrantes-Freer, Dr. Alexander Opitz and Prof. Dr. Andrea Antal for teaching me so many different methods and for helping me out with experimental designs.

Speaking of experiments, I would have never been able to perform so many without the excellent help of our laboratory assistants Olga, Heidi, Brigitte, Katja, Angela, Jasmin and Giselheid. Further thanks go to Uta and Mareike for teaching me several aspects of immunohistochemical stainings. Moreover, special thanks to Claudia and Anne for their helpful comments on this manuscript.

Furthermore, I would like to thank my colleagues from the Department of Clinical Neurophysiology Ivan, Zsolt, Gabriel, Annika, Alex, Albert, Yuichiro, Manuel and Giorgi for the frequent support as well as the good times inside and outside of the clinic. Also, many thanks to the ladies of the Neuropathology Martina, Franzi, Verena, Claudia, Anne, Silke, Lena, Nielsen, Nadine, Jana, Caro, Susi, Erika, Linda, Sarah, Kim and, of course, also to Basti, Darius as well as all other colleagues and friends. Thank you so much for all the great times, the scientific (and completely non-scientific) discussions and especially for accepting and welcoming the roomless guy from the Neurophysiology back in the days.

Finally, I would like to thank my parents Jutta and Martin for always supporting me and for all the trust and faith they have in me. Last but certainly not least, thank you Kathi for always being there for me and for your sympathy and love. 


\section{Abstract}

Multiple sclerosis (MS) is a chronic, inflammatory demyelinating disease of the central nervous system (CNS). Typical characteristics of MS are blood-brain barrier (BBB) breakdown, invasion of peripheral immune cells, demyelinated lesions and axonal damage with functional loss. Although the brain is able to remyelinate demyelinated lesions to a certain degree during the relapsing-remitting phase, for reasons yet unknown remyelination fails in the later, chronic stages of MS. The different disease stages of MS feature different pathological mechanisms, e.g. T cell infiltration and inflammatory activity decreases during the chronic phase, accompanied by a reduced remyelination capacity of the demyelinated lesions. Therefore, in this thesis, we evaluated the impact of CNS infiltrating and autoreactive immune cells on remyelination in a mouse model of demyelination.

We demonstrated that the induction of experimental autoimmune encephalomyelitis (EAE) by immunization with $\mathrm{MOG}_{35-55}$ peptide in a model of Cuprizone-induced demyelination led to increased infiltration of inflammatory mediators like T cells, B cells, NK cells, granulocytes and inflammatory monocytes and subsequent increased axonal damage. The infiltration of the CNS by auto-reactive immune cells however, did not substantially influence the remyelination process, as indicated by the analysis of oligodendrocyte populations, myelin formation and myelinated fiber density in the corpus callosum (CC).

To improve the efficacy of remyelination in demyelinating diseases like MS is of great therapeutic interest. Recent studies demonstrated neuroregenerative effects and increased (re-)myelination by electrical stimulation in mixed cortical cell cultures as well as rodent models of peripheral nerve injury and ischemic stroke. Based on these studies, we established a novel method for transcranial electrical stimulation in a rat model of focal, cortical demyelination as well as in a mouse model of chronic, Cuprizone-induced demyelination. Furthermore, we evaluated the impact of alternating current stimulation on oligodendrocyte survival, myelin production and the myelination of axonal fibers in oligodendrocyte and mixed cortical cultures.

The in vitro experiments did not reveal an impact of electrical stimulation on oligodendrocyte numbers and cell viability. Also, no effects on myelin production or the amount of myelinated fibers could be determined. However, we were able to apply transcranial alternating electrical currents safely and mostly non-invasively and therefore established the foundation for further experiments. However, the lack of effects on oligodendrocyte populations and remyelination, most likely caused by insufficient stimulation of neurons, indicate the need for improved stimulation protocols in terms of intensity and directionality. 
Theta burst stimulation (TBS), a form of repetitive transcranial magnetic stimulation (rTMS) established by Huang et al. in 2005, showed promising results in modulating neuronal activity of healthy human subjects, demonstrated by alterations of motor responses. Since the application of rTMS can be very uncomfortable for the recipient and bears an elevated risk of inducing epileptic seizures, we translated the established TBS protocols into electrical TBS (eTBS) protocols using high frequency, high intensity transcranial alternating currents.

A double-blinded, crossover clinical study on healthy human subjects was conducted, and eTBS proofed to be a safe and non-invasive way to apply high frequency, high intensity alternating currents to the human brain. However, no effects on cortical excitability were observed by analysis of muscle evoked potential (MEP) responses. 


\section{List of Figures}

Figure 1: MS pathogenesis and cytokine involvement.

Figure 2: Electrical currents in the CNS - influence on progenitor cell recruitment, OPC behavior and myelination.

Figure 3: Time scale of cuprizone treatment and EAE induction. 36

Figure 4: Time line of electrical stimulation in oligodendrocyte cultures and mixed cortical cultures.

Figure 5: Quantification of myelinated axons in mixed cortical cultures

Figure 6: Time line of the chronic cuprizone model.

Figure 7: Schematic overview of the four separate counting areas used to evaluate OLG populations in the focal EAE model.

Figure 8: Schematic illustration of the eTBS protocols......

Figure 9: 4.5 weeks of Cuprizone administration causes severe demyelination of the CC in Cup and Cup-EAE mice. 59

Figure 10: Immunization with $\mathrm{MOG}_{35-55}$ peptide leads to increased infiltration of immune competent cells.

Figure 11: Less mature OLG in the medial corpus callosum at the RM time point after EAE induction.

Figure 12: The extent of de- and remyelination is unaffected by EAE induction. 65

Figure 13: The density of PLP-mRNA ${ }^{+}$cells is not affected by immunization with MOG $_{35-55}$ peptide.

Figure 14: Increased number of $\mathrm{APP}^{+}$axons in the lateral $\mathrm{CC}$ of Cup-EAE mice.......

Figure 15: The density of myelinated axons is significantly reduced in Cup-EAE animals at the DM time point and not significantly different between the experimental groups at the RM time point.

Figure 16: $\mathrm{O}^{+}$oligodendrocytes and expression of MBP are unaffected by electrical stimulation. 72

Figure 17: ESTIM does not influence the amount of myelinated fibers. 73 
Figure 18: Cell survival is not influenced by electrical stimulation

Figure 19: Formation of a demyelinated lesion after stereotactical injection with Z2 antibody and mouse complement without substantial axonal damage and loss. 76

Figure 20: Lesion formation of sham and ESTIM animals over a time course of 21 days demonstrated by MBP immunohistochemistry.....

Figure 21: Demyelinated lesion size gradually decreases over a time course of three weeks.

Figure 22: Oligodendrocyte populations are largely unaffected by ESTIM. 81

Figure 23: Numbers of proliferating OLG are unaltered after ESTIM. 85

Figure 24: The extent of remyelination of ESTIM treated animals does not differ from sham treated animals. 86

Figure 25: ESTIM did not change the density of OPC after chronic demyelination 87

Figure 26: Numbers of NogoA $\mathrm{A}^{+}$cells are not altered by ESTIM. 88

Figure 27: Overall low number of proliferating OPC after 5 and 10 days..... 89

Figure 28: The amount of proliferating mature OLG is unaltered by ESTIM. 90

Figure 29: MEP amplitudes after eiTBS, eimTBS and ecTBS did not differ from sham. 93

Figure 30: MEP responses remain unchanged after stimulation with altered ecTBS protocols. 95

Figure 31: High interindividual variability of MEP amplitudes following eTBS and sham stimulation. 95 


\section{List of Tables}

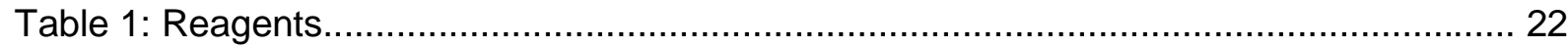

Table 2: Primary antibodies for immunohistochemical stainings.................................. 25

Table 3: Secondary antibodies for immunohistochemical stainings.................................. 26

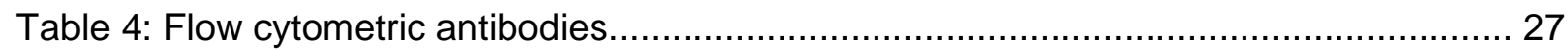

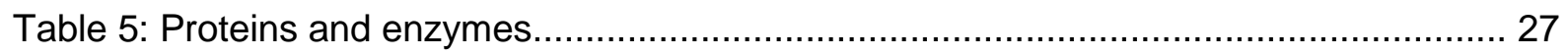

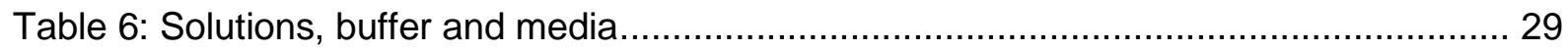

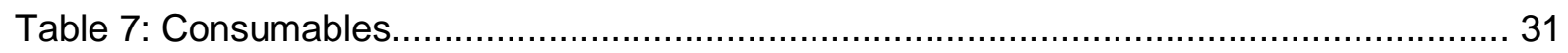

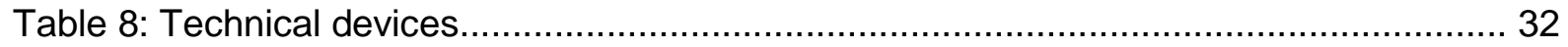

Table 9: Software

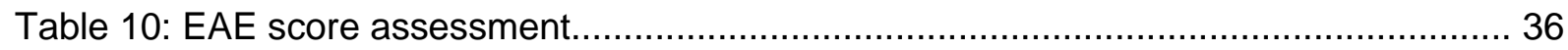

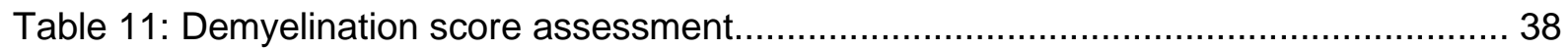

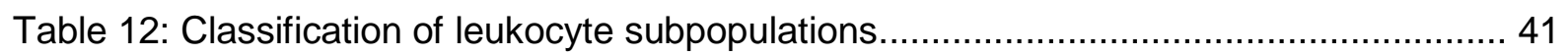




\section{Abbreviations}

AEC

Amino ethyl carbazol

ADCC Antibody dependent cellular cytotoxicity

AMPA a-amino-3-hydroxy-5-methyl-4-isoxazolepropionic acid

APAAP Alkaline phosphatase - anti alkaline phosphatase

APC Antigen presenting cell

APP Amyloid precursor protein

ATP Adenosine triphosphate

BBB Blood-brain barrier

BCIP 5-bromo-4-chloro-3-indolyl phosphate

BDNF Brain derived neurotrophic factor

BrdU Bromodeoxyuridin

BSA $\quad$ Bovine serum albumin

cAMP Cyclic adenosine monophosphate

CC Corpus callosum

CD Cluster of differentiation

CFA Complete Freund's Adjuvant

CNS Central nervous system

DAB 3,3'-Diaminobenzidine

DAPI 4',6-diamidino-2-phenylindole

DM Demyelination

DNA Deoxyribonucleic acid

DRG Dorsal root ganglion

EAE Experimental autoimmune encephalomyelitis

ecTBS Electrical continuous theta burst stimulation 


\begin{tabular}{|c|c|}
\hline EDTA & Ethylenediaminetetraacetic acid \\
\hline eimTBS & Electrical intermediate theta burst stimulation \\
\hline eiTBS & Electrical intermittent theta burst stimulation \\
\hline EM & Electron microscope \\
\hline ESTIM & Electrical stimulation \\
\hline eTBS & Electrical continuous theta burst stimulation \\
\hline FCS & Fetal calf serum \\
\hline FDI & First dorsal interosseous muscle \\
\hline GM & Gray matter \\
\hline HBSS & Hank's balanced salt solution \\
\hline $\mathrm{HCl}$ & Hydrochloric acid \\
\hline $\mathrm{HE}$ & Hematoxylin-Eosin \\
\hline i.p. & Intraperitoneal \\
\hline IFA & Incomplete Freund's adjuvant \\
\hline IFNy & Interferon- $y$ \\
\hline IL-17 & Interleukin-17 \\
\hline iNOS & Inducible nitric oxide synthase \\
\hline LDH & Lactate dehydrogenase \\
\hline LFB & Luxol fast blue \\
\hline LIF & Leukemia inhibitory factor \\
\hline MBP & Myelin basic protein \\
\hline MEP & Muscle evoked potential \\
\hline $\mathrm{MHC}$ & Major histocompatibility complex \\
\hline MOG & Myelin oligodendrocyte glycoprotein \\
\hline MS & Multiple sclerosis \\
\hline
\end{tabular}




\begin{tabular}{|c|c|}
\hline NAGM & Normal appearing gray matter \\
\hline NBT & 4-nitro blue tetrazolium chloride \\
\hline NF & Neurofilament \\
\hline NK cells & Natural killer cells \\
\hline NMDAr & $N$-methyl-D-aspartate receptor \\
\hline NogoA & Myelin-associated neurite growth inhibitor \\
\hline OLG & Oligodendrocyte \\
\hline OPC & Oligodendrocyte precursor cell \\
\hline PBS & Phosphate buffered saline \\
\hline PDGFR & Platelet derived growth factor \\
\hline PLP & Proteolipid protein \\
\hline PNS & Peripheral nervous system \\
\hline PPMS & Primary progressive multiple sclerosis \\
\hline PTX & Pertussis toxin \\
\hline RM & Remyelination \\
\hline RMT & Resting motor threshold \\
\hline RRMS & Relapsing-remitting multiple sclerosis \\
\hline rTMS & Repetitive transcranial magnetic stimulation \\
\hline SDS & Sodium dodecyl sulfate \\
\hline SPMS & Secondary progressive multiple sclerosis \\
\hline SVZ & Subventricular zone \\
\hline tACS & Transcranial alternating current stimulation \\
\hline TBS & Theta burst stimulation \\
\hline tDCS & Transcranial direct current stimulation \\
\hline tES & Transcranial electric stimulation \\
\hline
\end{tabular}


TGF $\beta \quad$ Tumor growth factor $\beta$

TMS Transcranial magnetic stimulation

TNFa Tumor necrosis factor $\alpha$

TTFields Tumor-treatment fields

TTX Tetrodotoxin

WM White matter 


\section{Introduction}

\subsection{Multiple sclerosis}

Multiple sclerosis (MS) is a chronic, inflammatory demyelinating disease of the central nervous system (CNS) which affects approximately 2.5 million people worldwide with a higher prevalence in women (HealthLine; Ahlgren et al., 2011). Although the exact cause of MS is still unknown, it is widely accepted to be a T cell driven auto-inflammatory disease (Noseworthy et al., 2000; Weiner, 2004). Speculations are made about genetic, cultural, environmental and behavioral impacts on the disease, since, e.g., people in the northern part of the northern hemisphere appear to be more susceptible to MS compared to people living in the equatorial area (WHO, Atlas: Multiple Sclerosis Resources in the World 2008). The heterogeneous disease course and prognosis of MS is another problem, which makes the care for people with MS very challenging. The typical disease course, with the onset placed between 25-30 years, starts with a pre-symptomatic disease with occasional neurological symptoms called clinically isolated syndrome (Eriksson et al., 2003). Often, patients with a clinically isolated syndrome develop relapsing-remitting MS (RRMS) in which inflammatory, demyelinating lesions occur in the white (WM) and gray matter (GM) of the CNS (Dendrou et al., 2015; Lassmann and Bradl, 2017). These lesions are frequently the cause for severe loss of motor and sensory function (Tallantyre et al., 2010). However, during RRMS the CNS is still able to remyelinate to a certain degree, whereby clinical symptoms might be reversed (Duncan et al., 2009). RRMS ultimately leads into a secondary progressive disease phase (SPMS). During SPMS, the severity of clinical disability increases without much evidence for ongoing inflammatory activity, whereas the remyelination capacity seems to decrease (Dendrou et al., 2015). Therefore, patients experience increased loss of body functions and a severe impairment in the quality of their life (Tallantyre et al., 2010).

Another form of MS is the primary progressive disease course (PPMS), which is often characterized by a more rapid disease progression. In PPMS, the axonal loss and thereby the functional impairment increases gradually without regenerative phases, as the like can be found in RRMS (Dendrou et al., 2015).

During each of the different MS phases, the molecular and cellular processes differ significantly and make a correct treatment challenging. For instance, the degree of $\mathrm{T}$ cell infiltration and therefore inflammation decreases in the later disease course (Kutzelnigg et al., 2005; Serafini et al., 2004) accompanied by a reduction of inflammatory active plaques, while at the same time, focal demyelinated white matter lesions are still present (Lassmann et al., 2007). Highly effective drugs like rituximab, alemtuzumab or dimethyl fumarate work almost exclusively by reducing lymphocyte numbers. Therefore, while effectively reducing the relapse rate and increasing the time to progression of disability during RRMS, they prove 
to be less effective during the progressive disease course (Coles et al., 2006; Hauser et al., 2013; Hawker et al., 2009). During primary and secondary progressive MS, it is of foremost importance to improve neuroprotection and neuroaxonal repair to prevent neurodegeneration caused by the underlying disease process.

\subsubsection{MS pathology}

The main hallmarks of MS are blood-brain barrier (BBB) breakdown, invasion of peripheral immune cells, primary demyelinated lesions with subsequent oligodendroglial demise as well as neuroaxonal damage and loss. The infiltration of autoreactive $T$ and $B$ cells through the BBB into the CNS is a phenomenon which occurs early in the disease development and is believed to be a major cause of disease pathology (Fig. 1; Noseworthy et al., 2000; Popescu and Lucchinetti, 2012; Weiner, 2004). It was discovered that circulating $\mathrm{CD}^{+} \mathrm{T}$ cells from MS patient were recognizing the basic myelin proteins proteolipid protein (PLP), myelin oligodendrocyte glycoprotein (MOG) and myelin basic protein (MBP), therefore indicating that the demyelinated lesions might be caused by an autoimmune response following autoantigen presentation in the periphery (Bielekova et al., 2004). However, $\mathrm{CD} 4^{+} \mathrm{T}$ cells obtained from healthy controls were found to occasionally display similar results (Hellings et al., 2001).

Autoreactive $\mathrm{CD}^{+} \mathrm{T}$ cells in MS patients consist mostly of the subtypes $\mathrm{T}_{\mathrm{H}} 1$ and $\mathrm{T}_{\mathrm{H}} 17$, producing the pro-inflammatory cytokines interferon- $\mathrm{Y}$ (IFNY) and interleukin-17 (IL17), respectively (Cao et al., 2015; Goverman, 2009). Once inside the CNS, it is believed that pro-inflammatory cytokines like IFNy, IL-17, IL-1 1 , IL-2 and tumor necrosis factor $\alpha$ (TNF $\alpha$ ) facilitate an immune reaction and further weaken the BBB (Minagar and Alexander, 2003). IFNY and IL-17 promote the activation of antigen presenting cells, represented in the CNS by astrocytes, microglia and macrophages, while IL-1 $\beta$ is mostly expressed by microglia and infiltrating macrophages and can induce oligodendrocyte death in vitro (Martins et al., 2011; Takahashi et al., 2003). IL-2 in turn, is released by $\mathrm{CD} 4^{+}$and $\mathrm{CD} 8^{+} \mathrm{T}$ cells after reactivation with antigen presenting cells and leads to further $\mathrm{T}$ and $\mathrm{B}$ cell proliferation as well as IgG2 antibody production (Martins et al., 2011). TNFa is another cytokine released by $C D 4^{+} T_{H} 1 T$ cells and is assumed to play a role in BBB breakdown, demyelination and oligodendrocyte damage (Duchini et al., 1996; Ledeen and Chakraborty, 1998; Takahashi et al., 2003). The inflammatory milieu caused by proinflammatory cytokines as well as a weakening of the BBB results in further autoreactive lymphocytes and serum factors entering the CNS. Among the infiltrates, autoreactive $\mathrm{CD}^{+} \mathrm{T}$ cells dominate but also B cells can be found (Frischer et al., 2009). The $\mathrm{CD}^{+} \mathrm{T}$ cell numbers were found to closely correlate with axonal damage (Booss et al., 1983; Frischer et al., 2009; Hayashi et al., 1988). While it is assumed that infiltrating $\mathrm{CD}^{+} \mathrm{T}$ cells are the major source of CNS inflammation during the initial phase of MS, CD8 ${ }^{+}$ 
T cells are predominantly found during the ongoing disease and presumably are key players for inflammation and tissue damage during the later phases of MS (Lassmann and Bradl, 2017). Similarly, the number of B cells also varies during MS development. For instance, an increased number of antibody-secreting plasma cells was discovered in patients with progressing PPMS or SPMS compared to RRMS (Frischer et al., 2009).

Activated microglia and macrophages express nitric oxide and reactive oxygen species like myeloperoxidase (e.g. during myelin phagocytosis)(Gray et al., 2008; Marik et al., 2007). Although the exact mechanisms behind neurodegeneration in MS remain mostly elusive, several studies demonstrated mitochondrial injury and impairment due to DNA mutation following exposure to raised levels of reactive oxygen and nitric oxide (Campbell et al., 2012; Fischer et al., 2012; Haider et al., 2011). Oligodendrocytes (OLG) are responsible for producing vast amounts of myelin and enwrapping nearby axons in the CNS and therefore require a high metabolic rate and, as a result, are very susceptible to mitochondrial damage. Thereby, it appears plausible that OLG are severely affected by an extended increase in inflammation and mitochondrial stress induced by reactive oxygen species.

Accompanying the loss of myelin sheaths, MS lesions often depict axonal degeneration and synaptic dysfunction (Jürgens et al., 2016; Popescu and Lucchinetti, 2012). Interestingly, a recent study demonstrated that an experimental delay of axonal degeneration in an inflammatory, demyelinating mouse model of MS did not ameliorate the degree of axonal damage (Singh et al., 2017). Thus, indicating a more intricate mechanism behind axonal degeneration, including impairment of axonal transport and intra-axonal $\mathrm{Ca}^{2+}$ concentrations (Singh et al., 2017). The impairment of axonal activity due to the lack of trophic support by extended demyelination or by axonal damage might, among other factors, result in synaptic loss (Albert et al., 2016). 


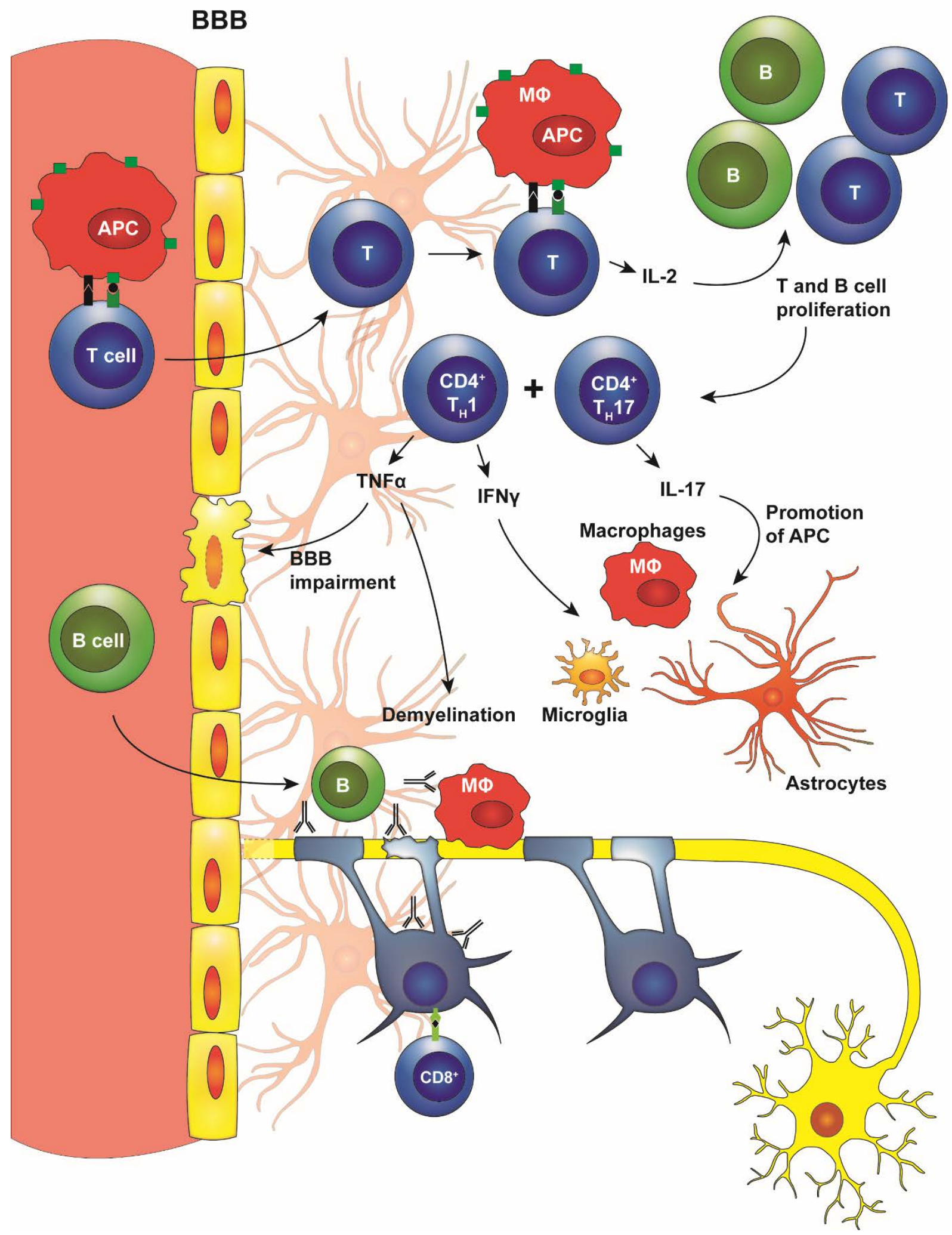


Figure 1: MS pathogenesis and cytokine involvement (continued from previous page)

It is assumed that autoreactive $T$ cells are activated by antigen presenting cells in the periphery and subsequently infiltrate the CNS through the BBB. Presumably, the initial phase of MS is mostly driven by infiltrating $C D 4^{+} T$ cells consisting of the subtypes $T_{H} 1$ and $T_{H} 17$, which are producing proinflammatory cytokines like IL-2, IL-17, IFNy or TNFa. The cytokine release can lead to increased T and B cell proliferation, activation of CNS antigen presenting cells, BBB impairment as well as demyelination. During the later stage of $\mathrm{MS}, \mathrm{CD}^{+} \mathrm{T}$ cells are predominantly involved in spreading inflammation and tissue damage. Activated B cells transform into plasma cells and produce myelin specific antibodies, which ultimately leads to phagocytosis of myelin sheaths by macrophages and microglia. Abbreviations: APC antigen presenting cells, BBB blood brain barrier, $\mathbf{M} \Phi$ macrophage.

\subsubsection{MS lesion histopathology}

The loss of myelin, as observed in MS, manifests in demyelinated lesions, which not only occur in the white matter but also in the cerebral and cerebellar cortex as well as in the deep gray matter. Interestingly, MS appears to be the only demyelinating disease demonstrating subpial cortical lesions (Fischer et al., 2013), which can be characterized into subpial, intracortical, leukocortical and pancortical lesions, depending on the respective location (Bø et al., 2003; Kuhlmann et al., 2017). The lesion formation in MS is a very dynamic process and is accompanied by infiltration of macrophages, microglia as well as $T$ and B cells. In the case of acute demyelination, microglia and macrophages clear the lesion of myelin debris by phagocytosis (Mosley and Cuzner, 1996). Therefore, newly formed MS lesions usually depict a high density of macrophages/microglia with incorporated fragments of myelin (Kuhlmann et al., 2017). Since it seems that the number of macrophages and microglia in MS lesions changes more dynamically compared to the amount of $\mathrm{T}$ cells and $\mathrm{B}$ cells, Kuhlmann et al. suggested a new MS lesion classification system in 2017 (Kuhlmann et al., 2017). The commonly used term "active lesion" refers to the most abundant lesion type found in RRMS patient, featuring loss of myelin, severe infiltration with microglia and/or blood-derived macrophages as well as T cells (Kuhlmann et al., 2017; Kutzelnigg et al., 2005). Active lesions can also be found in SPMS patients, although less frequently (Kutzelnigg et al., 2005). The new classification system by Kuhlmann et al. separates active lesions into active and demyelinating as well as active and post-demyelinating lesions (Kuhlmann et al., 2017). The active/demyelinating lesions are characterized by the presence of macrophages/microglia containing myelin degradation products such as MBP or PLP. Active/post-demyelinating lesions on the other hand, only show foamy macrophages/microglia, containing unspecific lipids instead of myelin debris. Moreover, Kuhlmann and colleagues distinguished active lesions from mixed active/inactive lesions, which are further separated into mixed active/inactive and demyelinating as well as postdemyelinating lesions. The main differences in mixed active/inactive compared to active 
lesions are a hypocellular lesion center and a lesion border marked by activated macrophages/microglia (Kuhlmann et al., 2017). Demyelinating mixed active/inactive lesions, as per Kuhlmann et al., can again be discriminated from post-demyelinating mixed lesions by the presence of macrophages/microglia actively digesting myelin fragments. Inactive lesions, finally, can be distinguished from active and mixed active/inactive lesions by their hypocellular nature and almost complete depletion of oligodendrocytes as well as the lack of a macrophage/microglia rim at the lesion border (Kuhlmann et al., 2017). According to Kuhlmann and colleagues, all of the before mentioned lesion types may show signs of remyelination. However, extensive remyelination can only be observed in approximately $20 \%$ of the lesions (Goldschmidt et al., 2009; Patrikios et al., 2006).

The impaired remyelination is a fundamental problem in MS and I will describe the mechanisms behind (re-)myelination as well as potential treatment approaches in 1.2. Beforehand, however, I will describe relevant MS animal models, which helped many discoveries regarding $\mathrm{MS}$ in the past decades, and discuss their advantages and shortcomings.

\subsubsection{MS animal models}

\subsubsection{Experimental autoimmune encephalomyelitis}

Although there are several different animal models for MS, none is truly able to mirror all the different facets of MS. The most frequently used model to study the inflammatory and immunological aspects of MS is experimental autoimmune encephalomyelitis (EAE).

The origin of EAE leads back to 1925, when Koritschoner and Schweinburg immunized rabbits with human spinal cord homogenate and induced spinal cord inflammation and subsequently paralysis (Koritschoner and Schweinburg, 1925). To improve reproducibility, Kabat et al. introduced the addition of complete Freund's adjuvant (CFA), consisting of inactivated mycobacteria, which significantly amplified the immune response by increasing the phagocytic uptake and antigen presentation (Billiau and Matthys, 2001; Kabat et al., 1951). Out of it evolved the EAE model as it is known today, with many different application forms in different vertebrates. EAE can be induced in mice, rats and primates (and other species) by immunization against myelin associated proteins and peptides like e.g. myelin oligodendrocyte glycoprotein MOG $_{35-55}$ peptide (Mendel et al., 1995). Although EAE was originally introduced in rabbits and later in rats, today it is mostly performed in mice, primarily due to a variety of genetically modified strains as well as less animal keeping costs and easier handling (Croxford et al., 2011). Similar to MS, this model displays infiltration of the CNS by activated lymphocytes, a breach of the BBB and axonal damage, which however, mainly takes place in the spinal cord. Contrary to MS, the inflammatory mediators are mostly autoreactive major histocompatibility complex (MHC) class II restricted 
$\mathrm{CD}^{+} \mathrm{T}$ cells which orchestrate mainly primary axonal damage with prevailing secondary demyelination (Kim et al., 2006; Nikić et al., 2011; Soulika et al., 2009), while in MS generally a primary demyelination with secondary axonal damage is displayed. The immunization of mice with $\mathrm{MOG}_{35-55}$ peptide and CFA is usually accompanied by injections with pertussis toxin (PTX) (Hofstetter et al., 2002).

This active EAE model generally displays an ascending paralysis, starting at the tail (disease onset 10-14 days after immunization), followed by impairment/paralysis of the hind limbs, the abdominal muscles and ultimately the forelimbs. Similar to progressive MS, EAE induction in C57BI/6 mice leads to a monophasic disease course with infrequent remission and chronic neurological impairment.

In recent years, many alternative EAE models were established. Among these are models like the adoptive transfer of $T$ cells as well as animals that develop a spontaneous EAE due to genetic manipulation. The former extracts myelin-antigen specific $T$ cells from a donor animal and transfers them into a naïve recipient animal to induce brain inflammation (Ben-Nun et al., 1981; Flügel et al., 2001). The spontaneous EAE model, on the other hand, uses transgenic animals which express $T$ cell receptors specific for myelin proteins like MOG (Bettelli et al., 2003). Up to $80 \%$ of double transgenic mice develop a spontaneous EAE several months after birth (Pöllinger et al., 2009). However, similar to the classic EAE, the inflammation is driven mainly by $\mathrm{CD} 4^{+} \mathrm{T}$ cells.

\subsubsection{Toxic demyelination by Cuprizone administration}

Different aspects of MS can be studied by models of toxic demyelination. Several models were established in recent years, utilizing substances like Cuprizone, lysolecithin or ethidium bromide. Cuprizone, one of the most frequently used toxic agents, is administered by mixing the cuprizone powder with animal chow. Most commonly, it is fed to C57BI/6 mice and leads to demyelination by oligodendrocyte death if administered for four to six weeks, depending on the Cuprizone dose and the age at the time of application (Hiremath et al., 1998; Matsushima and Morell, 2001). Once the mice are put on normal chow, they remyelinate over a time course of several days to weeks up to an almost full recovery.

The exact mechanisms behind the oligodendrocyte death remain largely unknown, although it is assumed that Cuprizone, being a copper chelator, exerts its effects by depriving oligodendrocytes (and all other cells) of copper. Copper is a trace element required by cytochrome oxidase, which plays a crucial role in oxidative phosphorylation. Copper deficiency results in enlarged, bloated and subsequently impaired mitochondria in the liver, thus demonstrating a severe impact on cell metabolism (Suzuki, 1969; Suzuki and Kikkawa, 1969). Since oligodendrocytes entertain a high metabolic rate to provide axons with vast amounts of myelin as well as trophic support, it is believed that they are especially 
susceptible to metabolic stress caused by the lack of copper (Matsushima and Morell, 2001). However, re-administering copper to Cuprizone treated mice failed to reduce oligodendrocyte death and demyelination (Carlton, 1967).

Although the demyelination followed by remyelination in the Cuprizone model provides a good opportunity to study the molecular backgrounds of de- and remyelination, the spontaneous remyelination phase following Cuprizone treatment occurs very rapidly and displays an extensive recovery. Therefore, short-termed Cuprizone feeding does not mirror the impaired remyelination observed in PPMS and SPMS. To deal with this problem, the chronic Cuprizone model was established (Matsushima and Morell, 2001). Here, mice were fed Cuprizone for an extended period of 12 weeks and showed an impaired remyelination for at least 6 weeks (Harsan et al., 2008; Hussain et al., 2013).

In contrast to MS, the immune response after Cuprizone administration is mainly driven by microglial and astrocytic response, limiting the comparability to MS (Carlton, 1967; Hiremath et al., 1998). To study demyelination under the influence of infiltrating autoreactive immune cells, a new animal model including inflammatory $T$ cells should be established.

\subsubsection{Cuprizone-EAE model}

One way to address the multi-faceted symptoms of MS would be to combine the Cuprizone model with the phenotypic CNS demyelination with the adaptively-driven EAE as it was done in the doctoral thesis of Angelika Escher in 2008 and by Boretius and colleagues in 2012 (Boretius et al., 2012). As previously mentioned, the degree of inflammation in MS decreases in the later, progressive disease course (Kutzelnigg et al., 2005; Serafini et al., 2004). This correlates with an impairment in remyelination and increased neurodegeneration (Noseworthy et al., 2000), thus provoking the thought that a certain inflammatory milieu might support remyelination (Foote and Blakemore, 2005). On the other hand, Lin et al. reported in 2006 that IFNy, a pro-inflammatory cytokine produced by activated T cells and natural killer cells in MS, effectively impaired remyelination in Cuprizone and EAE animals (Lin et al., 2006).

The immunization of Cuprizone treated mice with $\mathrm{MOG}_{35-55}$ peptide in combination with PTX and thereby activation of the adaptive immune system in a model of CNS demyelination might shed some light on the impact of inflammatory mediators on remyelination. So far, A. Escher was able to demonstrate that the induction of $E A E$ in Cuprizone animals led to an increase of inflammatory cells like $\mathrm{CD}^{+} \mathrm{T}$ cells, microglia and macrophages as well as increased expression of the inflammatory markers iNOS, MHC class II and IFNy. However, no difference was discovered between Cuprizone-EAE and Cuprizone animals by analysis of myelin- and oligodendrocyte specific stainings and oligodendrocyte related genes in the corpus callosum (CC). 


\subsubsection{Focal demyelinated lesions}

The focal stereotactical injection of myelin specific antibodies or pro-inflammatory cytokines represents another way to study primary demyelination in white or gray matter lesions. In 2006, Merkler et al. established a method to target EAE lesions to the cortex. Demyelinated lesions were created by cortical injection of TNFa and IFNy in rats subclinically immunized with recombinant $\mathrm{MOG}_{1-125}$ protein (Merkler et al., 2006). Thereby, demyelinated cortical lesions with $\mathrm{T}$ cell as well as microglia/macrophage involvement were induced, resembling cortical lesions observed in MS patients (Kutzelnigg et al., 2005). Furthermore, these cortical demyelinated lesions underwent rapid and extensive remyelination (Merkler et al., 2006).

A further advantage of stereotactical injections is the introduction of autoreactive antibodies into the CNS which otherwise would not be able to pass the BBB effectively (Saadoun et al., 2010, 2014; Wrzos et al., 2014). For instance, in 2014 Wrzos et al. demonstrated that the injection of recombinant anti-aquaporin 4 auto-antibodies of NMO patients in combination with human complement produced astrocyte-depleted lesions with secondary oligodendrocyte death and demyelination, therefore elucidating different mechanisms regarding demyelination in MS and NMO (Wrzos et al., 2014). Consequently, the stereotactical injection of demyelinating agents into the CNS offers promising opportunities to study de- and remyelination with and without activation of the immune system in a controlled and reproducible manner.

\subsection{Myelination}

Myelin is a complex structure, which is formed by mature oligodendrocytes in the CNS and by Schwann cells in the peripheral nervous system (PNS) to provide metabolic and mechanical support for axons and to improve nerve conduction by a process called "saltatory conduction". Oligodendrocytes form sheaths of myelin around nearby axons, which is a major component of the white matter in humans $(50-60 \%$ dry weight of the white mater)(Morell and Norton, 1980) and was first discovered by Virchow in 1854 (Virchow, 1854). Recent studies indicated that alongside improved nerve conduction, myelin or more precisely oligodendrocytes and Schwann cells also provide metabolic and trophic support for myelinated axons (Beirowski et al., 2014; Fünfschilling et al., 2012; Lee et al., 2012; Morrison et al., 2013). For instance, in 2012 Lee et al. demonstrated that the lack of lactate transport by the lactate transporter monocarboxylate transporter 1 , localized in myelin sheaths around CNS axons, led to axonal dysfunction and neurodegeneration (Lee et al., 2012).

Most of the myelination during human development takes place during the first year of life and continues throughout young adulthood (Fields, 2008). The ability of the CNS for myelination remains during the entire life and is directly linked to brain plasticity, as 
demonstrated by a process called "adaptive myelination". It explains how experiences like learning new motor or cognitive skills can have a direct effect on white matter volume, not only during development (Bengtsson et al., 2005) but also in healthy adults (Schlegel et al., 2012; Scholz et al., 2010). Although the formation of new myelin sheaths is very important, it is crucial to control myelination so as not to myelinate, for instance, inactive axons, axons, which are already myelinated or the full length of an unmyelinated axon. This is accomplished by complex processes, which are not fully understood yet. In vitro studies suggest that electrical activity from axons might lead to the release of neurotransmitters such as ATP and glutamate which in turn influence oligodendrocyte calcium levels and modulate MBP protein synthesis (Wake et al., 2011). These findings could explain the increase in WM volume observed during adaptive myelination. Furthermore, ligands expressed by axons like Jagged, polysialylated-neural adhesion cell molecule and Lingo1 were discovered to inhibit either oligodendrocyte precursor cell (OPC) differentiation or myelination (Charles et al., 2000; Mi et al., 2005; Wang et al., 1998). Moreover, during the enwrapping process the axonal and glial surfaces have to communicate with each other in order to sustain the contact. In this, the axoglial adhesion complex consisting of contactin-1 and the contactinassociated protein 1 seem to play a crucial role on the axon with the counterpart neurofascin on the glia surface (Pedraza et al., 2009; Zonta et al., 2008).

Different models exist to describe the method by which oligodendrocytes enwrap axons in the CNS, e.g. the "jelly roll" model (Bunge et al., 1961), the "serpent" model (Pedraza et al., 2009), the "liquid croissant" model (Sobottka et al., 2011) or the "ofiomosaic" model (Ioannidou et al., 2012). A recent study indicated that oligodendrocytes might myelinate axons with a single triangular-shaped membrane sheath which wraps around the axon by the foremost leading edge going underneath the previously produced layer and simultaneously expanding laterally (Snaidero et al., 2014). The myelin sheath extends laterally until it forms paranodal loops with the help of the before mentioned proteins contactin-1, contactin-associated protein 1 and neurofascin, thus creating the nodes of Ranvier. Since the frequency and length of internodes on each axon plays an important role in neuronal coupling and conduction velocity (Waxman, 1997; Wu et al., 2012), a controlled myelination is crucial. Oligodendrocytes, being the myelin producing cells in the CNS, are one of the key players in orchestrating those mechanisms, therefore oligodendrocyte development and maturation is regulated by a complex set of factors.

\subsubsection{Oligodendrocyte development}

Oligodendrocytes have to differentiate from oligodendrocyte precursor cells into immature pre-myelinating oligodendrocytes and subsequently into mature oligodendrocytes before they are able to enwrap axons with myelin sheaths. During early development, initial 
waves of OPC are generated in the ventral part of the neural tube, which changes to the dorsal area of the neural tube during the late developmental course (Penfield, 1924). But OPC are continuously produced throughout adulthood as well, originating from neural stem cells in the subventricular zone (SVZ) (Menn et al., 2006), as well as from NG2 glia cells, which can be found throughout the CNS and seem to remain in a slowly proliferative state (Dawson et al., 2003; Simon et al., 2011; Young et al., 2013). Regardless of the developmental stage, OPC typically proliferate and migrate through the CNS before reaching a target site and differentiating into mature myelinating oligodendrocytes. The process of differentiation is tightly regulated by numerous genes, transcription factors and neurotransmitters. Following, I will summarize a few of the regulators, which are vital for the differentiation from OPC into mature myelinating oligodendrocytes.

The transcription factor Olig2, for instance, is expressed in oligodendrocytes throughout their development, primarily in OPC and premature oligodendrocytes, thus serving as a prominent oligodendrocyte lineage marker. Olig2 is crucial for the development of oligodendrocytes as was shown by Takebayashi in 2002 (Takebayashi et al., 2002). Further transcription factors like Hes5 (Liu et al., 2006), Sox5 and Sox6 (Stolt et al., 2006) or the receptor tyrosine kinase PDGFRa (Raff et al., 1988) were identified to prevent OPC from undergoing differentiation, therefore an inhibition of those factors with e.g. microRNA 219 and 338 is necessary to allow further differentiation (Dugas et al., 2011). As previously mentioned the ligand Jagged1, which is expressed by axons, also inhibits OPC maturation (Wang et al., 1998), while axonal activity might serve as a pro-differentiation stimulus. In this regard, recent studies indicated a correlation between the release of adenosine and/or adenosine triphosphate (ATP) by electrically active axons and improved differentiation of OPC and subsequent myelination (Ishibashi et al., 2006; Stevens et al., 2002). The utilization of DNA microarrays led to the discovery of the so called myelin gene regulatory factor, a specific transcription factor which is solely expressed by post mitotic oligodendrocytes and whose absence leads to a halt in the maturation process at a premyelinating state (Emery et al., 2009). Therefore, it can be summarized that the inactivation of several transcription factors and ligands, which keep OPC from undergoing differentiation with the simultaneous activation/expression of maturation-inducing transcription factors and cytokines is necessary to provide the development of OPC into mature myelinating oligodendrocytes (Liu and Casaccia-Bonnefil, 2010).

\subsubsection{Remyelination in MS}

As previously mentioned, the remyelination of demyelinated lesions is a phenomenon mostly observed in RRMS, which, however, eventually fails in the later, progressive disease course in many patients for reasons largely unknown (Dendrou et al., 2015; Franklin, 2002). 
This is dire, since remyelination seems to provide axonal protection and prevents axonal atrophy (Irvine and Blakemore, 2008; Kuhlmann et al., 2002). Improving remyelination is therefore a prime goal in the development of treatment strategies for demyelinating diseases like MS. A deeper understanding of efficient human remyelination, e.g. in RRMS, is a prerequisite.

First of all, remyelination in demyelinating diseases is presumably driven by OPC (Gensert and Goldman, 1997). A study performed by Prayoonwiwat and Rodriguez in 1993 using an animal model of experimental demyelination discovered that the amount of oligodendrocytes in the white matter was higher after remyelination compared to the oligodendrocyte number before demyelination (Prayoonwiwat and Rodriguez, 1993), therefore indicating that migrating OPC and not resident mature oligodendrocytes are crucial for remyelination. This claim is supported by two further studies: Target et al. demonstrated that mature myelinating oligodendrocytes were unable to form new myelin sheaths after being transplanted into areas of demyelination (Targett et al., 1996), while Keirstead and Blakemore showed in 1997 that post-mitotic oligodendrocytes, which survived in demyelinated areas despite having lost their myelin sheaths, lack the ability to enwrap nearby axons in newly formed myelin (Keirstead and Blakemore, 1997).

Two major sources were identified to contribute to the generation of new oligodendrocytes in response to demyelination: the subventricular zone and slowly proliferating NG2 glia cells (Dawson et al., 2003; Menn et al., 2006; Nait-Oumesmar et al., 1999; Young et al., 2013). NG2 glia cells appear to be the first line of defense in case of demyelination. They can be found throughout the CNS and are able to quickly form remyelinating oligodendrocytes (Dawson et al., 2003). Additionally, progenitor cells resident in the SVZ were found to contribute to remyelination by sending migratory precursor cells into the demyelinated lesion, which ultimately form remyelinating oligodendrocytes (Menn et al., 2006; Nait-Oumesmar et al., 1999).

The repopulation of demyelinated areas with newly formed, remyelinating oligodendrocytes is crucial for the regenerative phase in RRMS and other demyelinating diseases. In the chronic phase of MS, however, remyelination seems to fail. In contrast to earlier beliefs that this failure is caused by the total absence of OPC, it was discovered that OPC are present to a certain degree but somehow unable to proliferate and differentiate into remyelinating oligodendrocytes (Wolswijk, 1998). Stimulating OPC to proliferate and differentiate could therefore be a promising method to improve remyelination in chronic demyelinated lesions. 


\subsection{Electrical stimulation in neurodegenerative diseases}

Electrical stimulation as means to study nerve excitation and to treat neurological conditions is not a recent development but was used already over two centuries ago. As an example, the Italian physician Luigi Galvani (1737-1798) was among the first who utilized electrical stimulation to study nerve and muscle responses (Whittaker, 1910). Many scientists and physicians were following in his steps, and the methods of applying electrical currents in experimental models and clinical situations became more sophisticated. Since the nervous system is intrinsically driven by electricity, researchers were able to modulate cognitive processes like motor cortex excitability (e.g. Nitsche and Paulus, 2000) or working memory performance (e.g. Alekseichuk et al., 2016). However, also molecular and cellular mechanisms like cell proliferation and cytokine production were found to be influenced by electrical fields (Ishibashi et al., 2006; Kirson et al., 2007).

\subsubsection{Clinical application}

A way to apply electrical currents to deep brain structures is called deep brain stimulation. It has become a useful method in the treatment of Parkinson's Disease and epilepsy and can help patients with intractable variants of these diseases, in which established treatment methods fail (Morace et al., 2016; Volkmann et al., 2013). Deep brain stimulation requires, however, an invasive surgical implantation of electrodes into specific brain areas (e.g. the subthalamic nucleus, globus pallidus or ventral intermediate thalamic nucleus in case of Parkinson's Disease) (Muthuraman et al., 2017; Volkmann et al., 2013).

Entirely noninvasive clinical applications of electric currents in the brain, on the other hand, are the so-called tumor treatment fields (TTFields). TTFields consist of high frequency (100-200 kHz) alternating currents applied to patients with recurrent glioblastoma, generating electric fields of 1-2 V/cm (Kirson et al., 2007). Kirson et al. demonstrated that TTFields were able to reduce tumor cell growth in glioblastoma patients and, if used in combination with established chemotherapy, also increased the efficiency of the chemotherapy (Kirson et al., 2007, 2009). In vitro and in vivo experiments revealed that the unidirectional forces of high frequency alternating electric fields interfere with the spindle tubulin orientation during cell proliferation, therefore causing an arrest of cell proliferation and the death of dividing cells (Kirson et al., 2004). Although tumor cells appear to be very susceptible to TTfields due to their highly proliferative nature, a drawback of this method might be the fact that the proliferation of cells engaged in regenerative processes like, e.g. neuronal progenitor cells from the SVZ, would also be affected.

However, electrical stimulation can be applied in various ways, and many of them showed promising neuroregenerative effects. 


\subsubsection{Influence of electrical stimulation on neuronal progenitor cells}

Recent studies conducted on animal stroke models discovered beneficial effects of electrical stimulation on neuroregeneration by recruitment of neuronal progenitor cells (Fig. 2). For instance, striatal stimulation of rats in the chronic phase of a middle cerebral artery occlusion model with $100 \mu \mathrm{A}$ at $2 \mathrm{~Hz}$ led to an increased migration of proliferating neuronal progenitor cells from the SVZ towards the area of the ischemic stroke as well as an increased number of proliferating cells inside the ischemic penumbra (Morimoto et al., 2011). Similarly, Jahanshahi et al. demonstrated in 2013 that the stimulation of stroke rats with monopolar biphasic electrical currents of $100 \mu \mathrm{A}$ at frequencies of $30-330 \mathrm{~Hz}$ resulted in increased cell proliferation in the SVZ as well as enhanced migration of neuronal progenitors towards the area and an increased neurogenesis inside the area of ischemic stroke (Jahanshahi et al., 2013). Such findings formed the term "electrotaxis", which describes the process of increased migration along an electrical field. Earlier studies also demonstrated that neuronal progenitor cells migrated in a direct current field toward the cathode in an intensity dependent manner ( $\mathrm{Li}$ et al., 2008). Therefore, it seems that electrical currents, applied in a correct way, are able to influence the recruitment of neuronal progenitor cells, thus affecting tissue repair and regeneration.

\subsubsection{Peripheral nerve regeneration/remyelination by electrical stimulation}

Recognizing the potential of electrical stimulation, many studies performed experiments on rodent models of peripheral nerve injury. In this regard, Huang et al. demonstrated in 2013 the regenerative effects of short electrical stimulation (20 min of $3 \mathrm{~V}$ biphasic stimulation at $20 \mathrm{~Hz}$ ) on nerve recovery in a rodent model of sciatic nerve injury (Huang et al., 2013). After crushing the sciatic nerve, the application of biphasic electrical stimulation led to an increase of motor- as well as sensory neurons, which regenerated into the distal nerve stump, accompanied by an increase in nerve conduction velocity and a decrease of muscle atrophy. It was discussed that the regenerative effects were mediated by upregulation of regeneration-associated genes like $T$ - $\alpha 1$-tubulin or neurotrophic factors such as brain derived neurotrophic factor (BDNF) and its receptor tyrosine kinase trkB in neurons (Al-Majed et al., 2000; Huang et al., 2013). BDNF is secreted by electrically active axons and plays a central role in the axon-glia interaction in the peripheral nervous system (Chan et al., 2006; $\mathrm{Ng}$ et al., 2007). A study conducted by Wan et al. in 2010 verified the effect of electrical stimulation on BDNF expression: after stimulating the crush-injured sciatic nerve of rats with $20 \mathrm{~Hz}$ biphasic currents for one hour, Wan and colleagues observed increased levels of BDNF in stimulated rats for up to eight weeks, accompanied by an increased number of myelinated axons (Wan et al., 2010). 
Moreover, BDNF might be one of the key players, besides Neuregulin, in switching the oligodendrocyte mediated CNS myelination from a neuronal activity independent mode to a NMDA-receptor dependent and therefore neuronal activity dependent mode (Lundgaard et al., 2013). Hence, the CNS might also be a susceptible target for electrical stimulation in terms of remyelination.

\subsubsection{Influence of electrical stimulation on CNS remyelination}

The electrical stimulation of the CNS with the aim to provoke improved regeneration/remyelination in experimental models of focal demyelination represents one of the frontiers of brain stimulation. Not many studies were conducted on the complex central nervous system. In this line, Sherafat and colleagues demonstrated in 2013 that the stimulation of the rat CNS with electromagnetic fields in a model of focal toxic demyelination of the corpus callosum by lysolecithin led to an increase of cell proliferation, increased numbers of neuronal progenitor cells and improved remyelination in the demyelinated area (Sherafat et al., 2012). To improve remyelination in the CNS, electrical currents have to be able to influence oligodendrocyte differentiation and myelination in either a direct or indirect manner. As was demonstrated by in vitro studies, biphasic electrical fields of $10 \mathrm{~mA}$ intensity fluctuating at $10 \mathrm{~Hz}$ were able to increase the number of myelin producing oligodendrocytes, while reducing the number of apoptotic oligodendrocytes in oligodendrocyte/neuron cocultures as well as mixed cultures consisting of oligodendrocytes, astrocytes and neurons (Gary et al., 2012; Ishibashi et al., 2006; Stevens et al., 2002). The addition of the sodium channel inhibitor tetrodotoxin to the cultures prevented the effect, thus indicating that the improved oligodendrocyte survival and myelin production might be mediated by neuronal activity (Demerens et al., 1996; Gary et al., 2012).

Several theories exist concerning the mechanisms underlying electrically stimulated (re-)myelination. Stevens and colleagues proposed in 2002 that the signaling molecule adenosine acts as a neuron-glia transmitter to inhibit OPC proliferation, facilitate oligodendrocyte differentiation and subsequently improve myelin production (Stevens et al., 2002). Adenosine can be retrieved by the breakdown of extracellular ATP, which is released by electrically active axons (Fields and $\mathrm{Ni}, 2010$ ). Since OPC express adenosine receptors, they are able to recognize adenosine, thus responding directly to neuronal activity (Fig. 2, Stevens et al., 2002). Moreover, adenosine is not the only signaling molecule discussed as a neuron-glia transmitter. Similar results regarding improved myelination in mixed neuronal cultures were obtained by adding glutamate or leukemia inhibitory factor to the culture medium (Ishibashi et al., 2006; Wake et al., 2011). Glutamate, similar to ATP, is an important neurotransmitter and can diffuse into the extracellular space after vesicular release in synaptic clefts during neuronal activity (Bergles et al., 1999). It binds to a-amino-3-hydroxy-5- 
methyl-4-isoxazolepropionic acid (AMPA) receptors on OPC, which are able to depolarize in response due to $\mathrm{Ca}^{2+}$ influx, therefore demonstrating a rapid and direct signaling pathway between axons and OPC (Fig. 2, Bergles et al., 2000). Ishibashi and colleagues demonstrated in 2006 that astrocytes release the signaling molecule leukemia inhibitory factor (LIF) in response to extracellular ATP, released by electrically active axons (Fig. 2, Ishibashi et al., 2006). Leukemia inhibitory factor, in turn promotes myelination of mature oligodendrocytes.

In summary, neuronal activity and thereby electrical currents seem to play important roles in myelination and the promotion of remyelination, thus representing promising targets for the treatment of demyelinating conditions, experimentally and clinically. 


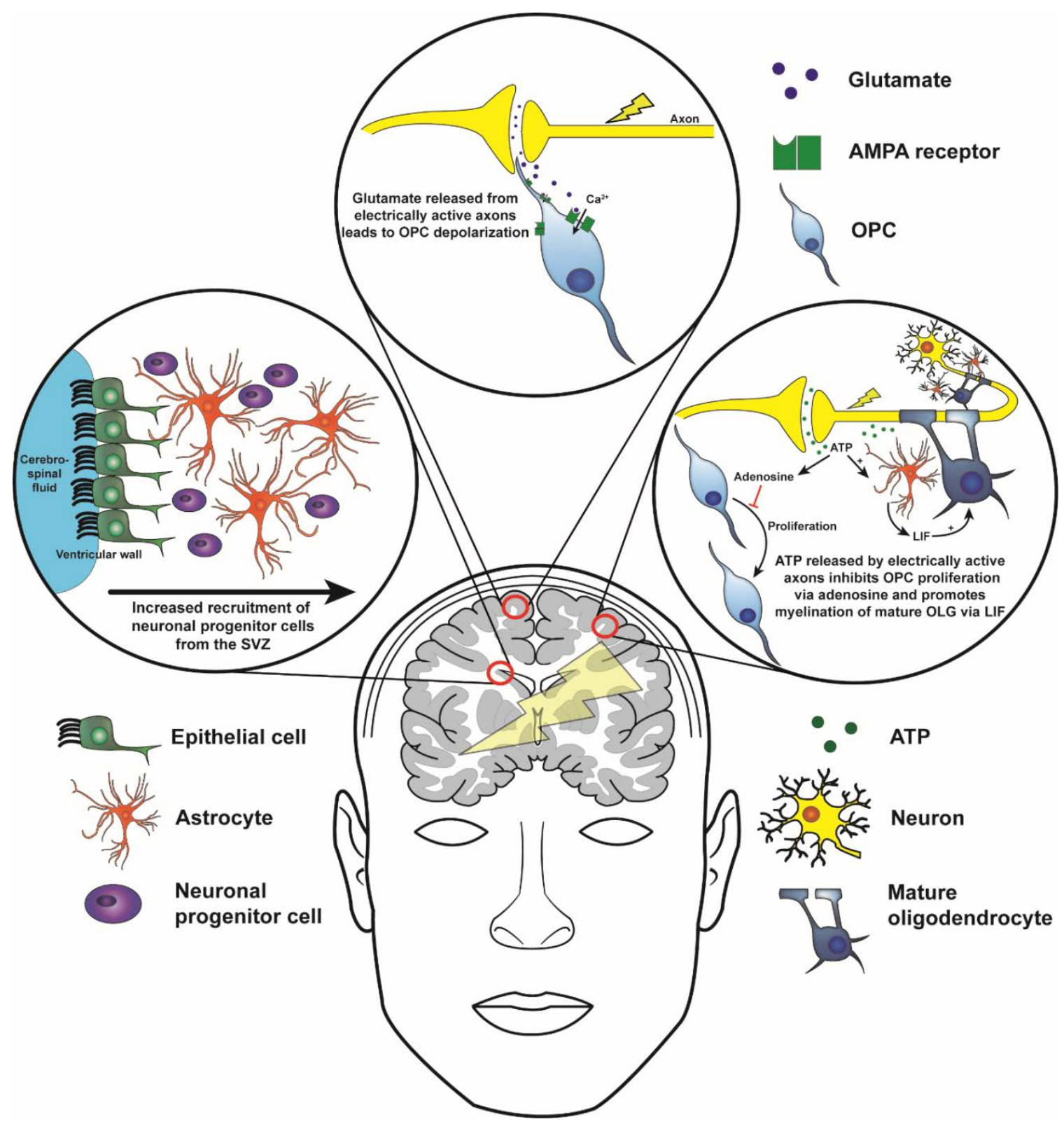

Figure 2: Electrical currents in the CNS - influence on progenitor cell recruitment, OPC behavior and myelination

Schematic illustration of several electrical cues that could lead to increased (re-)myelination in the CNS. It was proposed that electrical fields are able to increase the number of migratory, neuronal progenitor cells from the SVZ, which subsequently can differentiate into OPC (Jahanshahi et al., 2013; Morimoto et al., 2011). Furthermore, it was demonstrated that glutamate and ATP, released from electrically active axons, influence OPC behavior and myelination of mature oligodendrocytes. The binding of glutamate by AMPA receptors on OPC and subsequent depolarization of the cell by $\mathrm{Ca}^{2+}$ influx represents a rapid, direct axon-glia pathway (Bergles et al., 2000). The release of ATP can either inhibit OPC proliferation via an adenosinergic pathway (Stevens et al., 2002) or promote astrocytes to release leukemia inhibitory factor, which in turn promotes the myelin production of mature oligodendrocytes (Ishibashi et al., 2006). Abbreviations: AMPA $\alpha$-amino-3-hydroxy-5-methyl-4isoxazolepropionic, ATP adenosine tri-phosphate, LIF leukemia inhibitory factor, OLG oligodendrocyte, OPC oligodendrocyte precursor cell, SVZ subventricular zone. 


\subsection{Transcranial electrical stimulation}

A non-invasive way to introduce electrical currents into the brain, as opposed to the invasive deep brain stimulation, is the transcranial stimulation. Transcranial electrical stimulation (tES) comes in many different forms and application methods. The most frequently used methods are transcranial magnetic stimulation (TMS), transcranial direct current stimulation (tDCS) and transcranial alternating current stimulation (tACS). TMS, tDCS and tACS as well as many variations of those protocols are widely used in experimental and clinical studies to investigate structural and functional aspects of the human brain. Some tES protocols like TMS or tDCS are also applied for diagnostic purposes of neurological diseases or as means of treatment, e.g. in post-stroke recovery (Boggio et al., 2007; Khedr et al., 2005; Takeuchi et al., 2005).

\subsubsection{Transcranial magnetic stimulation}

TMS is a non-invasive brain stimulation technique utilizing a magnetic field of $1-2$ Tesla induced by a coil connected to a capacitor to establish an electrical field of up to 150 V/m for about $100 \mu$ s (Pascual-Leone et al., 2002). The electric field can be used to depolarize or hyperpolarize neurons in the underlying tissue in a painless manner. The use of TMS dates back to 1896, where D'Arsonval induced phosphenes (flashes of light caused by stimulation of the optic nerve) by stimulating the retina of volunteers (d'Arsonval, 1896). The first TMS device for clinical experimentation however, was introduced in 1985 by Barker et al. (Barker et al., 1985). The easy and safe application of TMS offered numerous opportunities to study structural and functional aspects of the human CNS and PNS. Depending on the clinical/experimental demands, different coil forms were created like the round coil for establishing a broad, diffuse electric field or the bent coil for occipital stimulation. The most frequently used coil shape in experimental studies is the figure-of-eight coil, due to the high focality of the induced electric field. This combination of two round coils creates the possibility to stimulate certain areas in the human cortex like the M1 region of the motor cortex.

With the help of monophasic TMS (one TMS pulse every four seconds), scientists and physicians can induce action potentials in, e.g. the M1 region of the motor cortex to provoke involuntary muscle contraction in the responding muscles. The amplitude of the muscle contraction can be measured by an electromyogram and is called "muscle evoked potential" (MEP). Measuring MEP represents an easy way to determine the current state of cortical excitability and can be used as a baseline in experimental setups to test the effects of tES interventions. Furthermore, MEP can be used in diagnostic situations to evaluate the nerve conduction in neurological diseases. 
A further application form of TMS is repetitive TMS (rTMS), featuring monophasic TMS pulses in a higher frequency. It is generally believed that high-frequency $r T M S(\geq 5 \mathrm{~Hz})$ results in an increase and low-frequency rTMS $(\leq 1 \mathrm{~Hz})$ results in a decrease of cortical excitability (Rothkegel et al., 2010). In clinically applications, it is used to treat neuropsychiatric diseases like chronic pain or depression (Lefaucheur et al., 2014; O'Reardon et al., 2007). The effects induced by rTMS are expected to be mediated by changes in synaptic plasticity following alterations of cortical excitability (Lenz et al., 2014; Noh et al., 2015; Trebbastoni et al., 2016; Vlachos et al., 2012). An effective way to modulate cortical excitability was demonstrated by Huang et al. in 2005 with the use of "theta burst stimulation" (TBS). TBS consists of 3 pulses repeated at $50 \mathrm{~Hz}$ and was applied in three different protocols: intermittent TBS (iTBS), intermediate TBS (imTBS) and continuous TBS (cTBS). Intermittent and continuous TBS induced facilitation and inhibition of cortical excitability, respectively, which outlasted the stimulation by 30-60 minutes (Huang et al., 2005). Although many studies were able to replicate this data (Gamboa et al., 2010; Huang et al., 2007; Di Lazzaro et al., 2005), a high intra- and interindividual variability is very common among the physiological responses of tES protocols (Hamada et al., 2013; Maeda et al., 2000).

\subsubsection{Transcranial alternating current stimulation}

tACS consists of oscillating electrical currents. In contrast to TMS, tACS usually does not directly induce action potentials in neurons but can entrain clusters of neurons, depending on the frequency used (Chaieb et al., 2011; Terney et al., 2008). Besides the frequency, the main parameters of tACS are stimulation intensity and waveform shape. Those can be altered in order to try to achieve different goals like, e.g., modulating ongoing rhythms in the cortex $(80-250 \mathrm{~Hz}, 1 \mathrm{~mA}$; Moliadze et al., 2010), provoking changes in corticospinal excitability (2-70 Hz, $1.5 \mathrm{~mA}$; Feurra et al., 2011), modulating voluntary movements in visuo-motor tasks $(20 \mathrm{~Hz}$, up to $0.7 \mathrm{~mA}$; Pogosyan et al., 2009) or even ameliorate carcinogenesis (100-200 kHz, 1-2 V/cm; Kirson et al., 2007). Furthermore, it was shown that transcranial alternating currents in the range of $2-5 \mathrm{kHz}$ could be applied safely with 1-2 mA intensity at the motor cortex and led to an increase in MEP amplitude for up to one hour (Chaieb et al., 2011), while the application of rTMS at such high frequencies exhibits a high risk of inducing epileptic seizures (Wassermann, 1998). It is generally believed that the modulatory effects of tACS below $1 \mathrm{kHz}$ are induced by entrainment of neuronal oscillations with an impact on cortical excitability as well as on learning (Alekseichuk et al., 2016), whereas tACS in the kilohertz range preferentially modulates the excitability of neuronal membranes (Antal and Paulus, 2013). Moreover, Cuellar and colleagues demonstrated in 2013 that the application of alternating currents with frequencies 
of $2-100 \mathrm{kHz}$ on the dorsal root ganglions of the exposed spinal cord inhibits sensory neurons, thus mediating a pain-relieving effect (Cuellar et al., 2013).

In summary, countless variations of tACS protocols were applied in experimental studies and many different outcomes were observed. Although many studies lack reproducibility, the easy non-invasive application and the high variability to which tACS protocols can be shaped allows for intensive study of cortical excitability and the influence of electric currents on molecular mechanisms.

\subsection{Aim of this thesis}

This thesis is divided into three parts. The first two parts address the question of how to improve remyelination in different rodent models of multiple sclerosis. The third part represents a clinical study, in which the safe application of high frequency/high intensity tACS protocols was evaluated in healthy human participants, with further regard to the modulation of cortical excitability.

\section{Part l. Influence of autoimmune inflammation on remyelination in Cuprizone-induced demyelination}

The inflammatory demyelinating disease multiple sclerosis features demyelinated lesions with substantial axonal damage and mostly, but not exclusively, during the early disease phase, a strong infiltration of autoreactive adaptive immune cells (Lassmann et al., 2007). The most commonly used MS animal models, the EAE and Cuprizone model, are only able to mirror certain aspects of MS. However, by combining these two models into a Cuprizone-EAE model, we can evaluate the impact of infiltrating, inflammatory cells and mediators on the remyelination of CNS demyelination. We thus immunized Cuprizone treated C57BI/6 mice with $\mathrm{MOG}_{35-55}$ peptide and determined the impact of infiltrating, autoreactive immune cells on axonal damage, oligodendrocyte populations and remyelination in the corpus callosum.

\section{Part II. Influence of transcranial alternating current stimulation on spontaneous remyelination in in vitro and in vivo models}

The potential of electrical currents to improve oligodendrocyte survival and increase (re-)myelination was demonstrated in cell culture experiments as well as animals models of peripheral nerve injury (Gary et al., 2012; Huang et al., 2013; Ishibashi et al., 2006; Wan et al., 2010). However, only a very limited number of electrical stimulation studies were conducted on models of CNS demyelination. Since the promotion of oligodendrocyte differentiation and the improvement of remyelination is a crucial factor in MS, especially 
during the progressive disease phase, the feasibility of transcranial electrical stimulation in this context should be thoroughly tested.

Therefore, in the second part of this thesis, we initially evaluated the impact of $10 \mathrm{~Hz}$, $10 \mathrm{~mA}$ alternating current stimulation on myelin production, oligodendrocyte survival and myelination of axonal fibers in oligodendrocyte as well as mixed cortical cultures. Subsequently, we applied alternating currents of $100 \mu \mathrm{A}$ at $10 \mathrm{~Hz}$ to different rodent models of demyelination in a transcranial fashion to determine the effect of electrical stimulation on lesion formation, degree of remyelination as well as OPC recruitment, proliferation and differentiation. The parameters of the stimulation protocols were chosen based on promising results of recent studies.

\section{Part III. Transcranial electrical stimulation in healthy human subjects}

The application of electrical currents to the human brain in a transcranial fashion provides a non-invasive method to modulate cortical plasticity. As demonstrated by Huang et al. in 2005, TBS protocols are able to either facilitate or inhibit neuronal excitation in the motor cortex, depending on the stimulation pattern (Huang et al., 2005). To induce high frequency rTMS pulses in the human brain, however, can be very uncomfortable for the participant and is connected to a greater risk of epileptic seizures (Wassermann, 1998). tACS on the other hand, can be safely applied in the low kilohertz range and does not lead to uncomfortable sensations on the recipients' skin when used with an intensity of 1-2 mA (Chaieb et al., 2011). Therefore, we introduced the electrical theta burst protocol (eTBS), which consists of high frequency, high intensity electric currents, applied in a TBS fashion. We choose a $5 \mathrm{kHz}$ "carrier frequency" in order to reduce pain perception and stimulation intensities of up to $10 \mathrm{~mA}$ to induce short-term changes in cortical excitability. The experiments were performed on healthy human volunteers in the setup of a clinical study published in Frontiers in Human Neuroscience 2017 (Kunz et al., 2017). 


\section{Methods}

\section{$2.1 \quad$ Materials}

Table 1 Reagents

\begin{tabular}{|c|c|}
\hline Reagent & Source of supply \\
\hline 4-nitro blue tetrazolium chlorid (NBT) & Sigma-Aldrich GmbH, Steinheim, Germany \\
\hline $\begin{array}{l}\text { 5-bromo-4-chloro-3-indolyl phosphate } \\
\text { (BCIP) }\end{array}$ & Sigma-Aldrich GmbH, Steinheim, Germany \\
\hline Acetic acid & Merck Millipore, Darmstadt, Germany \\
\hline Acetic anhydride & Merck Millipore, Darmstadt, Germany \\
\hline Amino ethyl carbazol (AEC) & Sigma-Aldrich GmbH, Steinheim, Germany \\
\hline Ammonia & Merck Millipore, Darmstadt, Germany \\
\hline B27 supplement & $\begin{array}{l}\text { Gibco, Life Technologies GmbH, Darmstadt, } \\
\text { Germany }\end{array}$ \\
\hline BME Medium & $\begin{array}{l}\text { Gibco, Life Technologies GmbH, Darmstadt, } \\
\text { Germany }\end{array}$ \\
\hline Calcium chloride $\left(\mathrm{CaCl}_{2}\right)$ & Merck Millipore, Darmstadt, Germany \\
\hline Chloral hydrate & Merck Millipore, Darmstadt, Germany \\
\hline Citric acid & Merck Millipore, Darmstadt, Germany \\
\hline Cuprizone & Sigma-Aldrich GmbH, Steinheim, Germany \\
\hline $\begin{array}{l}\text { Cytotox-One Homogenous Membrane } \\
\text { Integrity Assay }\end{array}$ & Promega, Madison, USA \\
\hline DAB (3,3'-Diaminobenzidine) & Sigma-Aldrich GmbH, Steinheim, Germany \\
\hline Dextran & Sigma-Aldrich GmbH, Steinheim, Germany \\
\hline $\begin{array}{l}\text { Disodium hydrogen phosphate dihydrate } \\
\left(\mathrm{Na}_{2} \mathrm{HPO}_{4} 2 \mathrm{H}_{2} \mathrm{O}\right)\end{array}$ & Merck Millipore, Darmstadt, Germany \\
\hline $\begin{array}{l}\text { DMEM (+4.5 g/l D-glucose, +L- } \\
\text { Glutamine, -Pyruvate) }\end{array}$ & $\begin{array}{l}\text { Gibco, Life Technologies GmbH, Darmstadt, } \\
\text { Germany }\end{array}$ \\
\hline Eosin G & Merck Millipore, Darmstadt, Germany \\
\hline
\end{tabular}




\begin{tabular}{|c|c|}
\hline Reagent & Source of supply \\
\hline Ethanol, $100 \%$ & Merck Millipore, Darmstadt, Germany \\
\hline Formaldehyde & Sigma-Aldrich GmbH, Steinheim, Germany \\
\hline FoxP3 fixation concentrate & eBioscience Ltd., Altrincham, UK \\
\hline FoxP3 fixation diluent & eBioscience Ltd., Altrincham, UK \\
\hline FoxP3 permeabilization buffer, 10x & eBioscience Ltd., Altrincham, UK \\
\hline Fluorescence mounting medium & $\begin{array}{l}\text { Dako Deutschland GmbH, Hamburg, } \\
\text { Germany }\end{array}$ \\
\hline Glutamax & Sigma-Aldrich GmbH, Steinheim, Germany \\
\hline Glutaraldehyde & Merck Millipore, Darmstadt, Germany \\
\hline Hank's balanced salt solution (HBSS) & $\begin{array}{l}\text { Gibco, Life Technologies GmbH, Darmstadt, } \\
\text { Germany }\end{array}$ \\
\hline Hydrochloric acid $(\mathrm{HCl})$ & Merck Millipore, Darmstadt, Germany \\
\hline Hydrogen peroxide $\left(\mathrm{H}_{2} \mathrm{O}_{2}\right), 30 \%$ solution & Merck Millipore, Darmstadt, Germany \\
\hline Isoflurane & Sigma-Aldrich GmbH, Steinheim, Germany \\
\hline Isoxylol & Merck Millipore, Darmstadt, Germany \\
\hline Ketamin, $10 \%$ & Medistar $^{\circledR}$, Ascheberg, Germany \\
\hline Lead citrate & Merck Millipore, Darmstadt, Germany \\
\hline Levamisole & Merck Millipore, Darmstadt, Germany \\
\hline Lithium carbonate & Merck Millipore, Darmstadt, Germany \\
\hline Luxol Fast Blue (LFB) & $\begin{array}{l}\text { BDH Laboratories Supplies, WWR Int. Ltd., } \\
\text { Poole, UK }\end{array}$ \\
\hline L-thyroxin solution & Merck Millipore, Darmstadt, Germany \\
\hline Mayer's hemalum & Merck Millipore, Darmstadt, Germany \\
\hline $\begin{array}{l}\text { Monastral Blue (Copper (II) } \\
\text { phthalocyaninetetrasulfonic acid } \\
\text { tetrasodium salt) }\end{array}$ & Sigma-Aldrich GmbH, Steinheim, Germany \\
\hline
\end{tabular}




\begin{tabular}{|c|c|}
\hline Reagent & Source of supply \\
\hline Naphthol As-Bi phosphate & Sigma-Aldrich GmbH, Steinheim, Germany \\
\hline Neufuchsin & Sigma-Aldrich GmbH, Steinheim, Germany \\
\hline Nitric acid, 65\% & Merck Millipore, Darmstadt, Germany \\
\hline NN-dimethylformamide & Sigma-Aldrich GmbH, Steinheim, Germany \\
\hline Osmium tetroxide $\left(\mathrm{OSO}_{4}\right)$ & $\begin{array}{l}\text { Carl Roth GmbH + Co. KG, Karlsruhe, } \\
\text { Germany }\end{array}$ \\
\hline Paraffin (Paraplast Plus ${ }^{\circledR}$ ) & Tyco Healthcare, Neustadt, Germany \\
\hline Paraformaldehyde & Merck Millipore, Darmstadt, Germany \\
\hline $\begin{array}{l}\text { Penicillin-streptomycin (10000 U/ml } \\
\text { penicillin; } 10 \text { mg/ml streptomycin) }\end{array}$ & Sigma-Aldrich GmbH, Steinheim, Germany \\
\hline Percoll ${ }^{\mathrm{TM}}$ & GE Healthcare, Uppsala, Sweden \\
\hline Periodic acid & Merck Millipore, Darmstadt, Germany \\
\hline Peroxidase-conjugated streptavidin & Sigma-Aldrich GmbH, Steinheim, Germany \\
\hline Phosphate buffered saline (PBS), 10x & Biochrom AG, Berlin, Germany \\
\hline Phosphate buffered saline (PBS), sterile & Sigma-Aldrich GmbH, Steinheim, Germany \\
\hline Poly-L-lysin & Sigma-Aldrich GmbH, Steinheim, Germany \\
\hline Propylenoxide & Merck Millipore, Darmstadt, Germany \\
\hline Renlam M-1 & $\begin{array}{l}\text { SERVA Electrophoresis } \mathrm{GmbH} \text {, Heidelberg, } \\
\text { Germany }\end{array}$ \\
\hline Silver nitrate $\left(\mathrm{AgNO}_{3}\right)$ & $\begin{array}{l}\text { Carl Roth GmbH + Co. KG, Karlsruhe, } \\
\text { Germany }\end{array}$ \\
\hline Sodium chloride $(\mathrm{NaCl})$, solution & Braun, Melsungen, Germany \\
\hline $\begin{array}{l}\text { Sodium dihydrogen phosphate } \\
\text { monohydrate }\left(\mathrm{NaH}_{2} \mathrm{PO}_{4} \mathrm{H}_{2} \mathrm{O}\right)\end{array}$ & Merck Millipore, Darmstadt, Germany \\
\hline Sodium dodecyl sulfate (SDS) & Merck Millipore, Darmstadt, Germany \\
\hline Sodium hydroxide solution $(\mathrm{NaOH})$ & Merck Millipore, Darmstadt, Germany \\
\hline Sodium nitrate & Merck Millipore, Darmstadt, Germany \\
\hline
\end{tabular}




\begin{tabular}{l|l}
\hline Reagent & Source of supply \\
\hline Sodium pyruvate solution (1.1\%) & Sigma-Aldrich GmbH, Steinheim, Germany \\
\hline Sodium thiosulfate pentahydrate & Sigma-Aldrich GmbH, Steinheim, Germany \\
\hline Temgesik (Buprenorphinhydrochloride) & Reckitt Benckiser, Slough, UK \\
\hline Toluidine Blue & Sigma-Aldrich GmbH, Steinheim, Germany \\
\hline Triiodthyronine solution & Merck Millipore, Darmstadt, Germany \\
\hline TritonX100 & MP Biomedicals, LLC, Santa Ana, CA, USA \\
\hline Trypan blue & Stemcell Technologies, Vancouver, Canada \\
\hline Uranylacetate & Merck Millipore, Darmstadt, Germany \\
\hline Xylazine solution, $20 \mathrm{mg} / \mathrm{ml}$ & Ecuphar, Oostcamp, Belgium \\
\hline Xylol & Merck Millipore, Darmstadt, Germany \\
\hline
\end{tabular}

Table 2 Primary antibodies for immunohistochemical stainings

\begin{tabular}{l|l|l|l|l|l}
\hline $\begin{array}{l}\text { Primary } \\
\text { antibody }\end{array}$ & Specificity & $\begin{array}{l}\text { Species } \\
\text { clone }\end{array}$ & Pretreatment & $\begin{array}{l}\text { Working } \\
\text { dilution }\end{array}$ & $\begin{array}{l}\text { Source of } \\
\text { supply }\end{array}$ \\
\hline APP & $\begin{array}{l}\text { Amyloid } \\
\text { precursor protein }\end{array}$ & $\begin{array}{l}\text { Mouse / } \\
22 \text { C11 }\end{array}$ & MW / C & $1: 2000$ & $\begin{array}{l}\text { Merck } \\
\text { Millipore, } \\
\text { Darmstadt, } \\
\text { Germany }\end{array}$ \\
\hline BrdU & $\begin{array}{l}\text { Bromodeoxyuridi } \\
\text { thymidine, used } \\
\text { as a proliferation } \\
\text { marker }\end{array}$ & Mouse & MW / C & $1: 400$ & $\begin{array}{l}\text { Merck } \\
\text { Darmstadt, } \\
\text { Germany }\end{array}$ \\
\hline MBP & $\begin{array}{l}\text { Myelin basic } \\
\text { protein }\end{array}$ & Rat & - & $1: 200$ & $\begin{array}{l}\text { Abcam, } \\
\text { Cambridge, } \\
\text { UK, Germany }\end{array}$ \\
\hline NF200 & Neurofilament & Rabbit & MW / C & $1: 100$ & $\begin{array}{l}\text { Merck } \\
\text { Millipore, } \\
\text { Darmstadt, } \\
\text { Germany }\end{array}$ \\
\hline
\end{tabular}




\begin{tabular}{|c|c|c|c|c|c|}
\hline $\begin{array}{l}\text { Primary } \\
\text { antibody }\end{array}$ & Specificity & $\begin{array}{l}\text { Species/ } \\
\text { clone }\end{array}$ & Pretreatment & $\begin{array}{l}\text { Working } \\
\text { dilution }\end{array}$ & $\begin{array}{l}\text { Source of } \\
\text { supply }\end{array}$ \\
\hline NG2 & $\begin{array}{l}\text { membrane } \\
\text { chondroitin } \\
\text { sulfate } \\
\text { proteoglycan; } \\
\text { oligodendrocyte } \\
\text { precursor cells }\end{array}$ & Rabbit & MW / C & $1: 200$ & $\begin{array}{l}\text { Merck } \\
\text { Millipore, } \\
\text { Darmstadt, } \\
\text { Germany }\end{array}$ \\
\hline NogoA & $\begin{array}{l}\text { Myelin- } \\
\text { associated } \\
\text { neurite growth } \\
\text { inhibitor; mature } \\
\text { oligodendrocytes }\end{array}$ & $\begin{array}{l}\text { Mouse / } \\
11 \mathrm{c} 7\end{array}$ & $\mathrm{MW} / \mathrm{C}$ & 1:15000 & $\begin{array}{l}\text { Kind gift from } \\
\text { M. } \\
\text { Schwab, } \\
\text { Zürich } \\
\text { (Oertle et al., } \\
\text { 2003) }\end{array}$ \\
\hline O4 & $\begin{array}{l}\text { Oligodendrocyte } \\
\text { lineage marker }\end{array}$ & Mouse & - & $1: 100$ & $\begin{array}{l}\text { R\&D Systems, } \\
\text { Abingdon, UK }\end{array}$ \\
\hline Olig2 & $\begin{array}{l}\text { Transcription } \\
\text { factor of } \\
\text { oligodendrocyte } \\
\text { precursor cells } \\
\text { and mature } \\
\text { oligodendrocytes }\end{array}$ & Rabbit & MW & $1: 300$ & $\begin{array}{l}\text { IBL } \\
\text { International } \\
\text { GmbH, } \\
\text { Hamburg, } \\
\text { Germany }\end{array}$ \\
\hline PLP & $\begin{array}{l}\text { Proteolipid } \\
\text { protein }\end{array}$ & Mouse & MW / C & $1: 250$ & $\begin{array}{l}\text { Biozol } \\
\text { Diagsnostica } \\
\text { GmbH, } \\
\text { Eching, } \\
\text { Germany }\end{array}$ \\
\hline TPPP/p25 & $\begin{array}{l}\text { Myelin protein; } \\
\text { mature } \\
\text { oligodendrocytes }\end{array}$ & Rabbit & MW / C & $1: 500$ & $\begin{array}{l}\text { Abcam, } \\
\text { Cambridge, } \\
\text { UK }\end{array}$ \\
\hline
\end{tabular}

Abbreviations: $\mathbf{C}$ citrate; $\mathbf{M W}$ micro wave

Table 3 Secondary antibodies for immunohistochemical stainings

\begin{tabular}{l|c|ll}
\hline Secondary antibody & Working dilution & Source of supply \\
\hline Alexa488 (Cy2) anti-mouse & $1: 200$ & $\begin{array}{l}\text { Thermo Fisher Scientific, Waltham, } \\
\text { MA, USA }\end{array}$ \\
\hline Alexa647 (Cy5) anti-rat & $1: 100$ & $\begin{array}{l}\text { Jackson ImmunoResearch Europe } \\
\text { Ltd., Suffolk, UK }\end{array}$ \\
\hline anti-mouse biotinylated & $1: 200$ & $\begin{array}{l}\text { GE Healthcare Europe GmbH, } \\
\text { Freiburg, Germany }\end{array}$ \\
\hline Anti-rabbit biotinylated & $1: 200$ & $\begin{array}{l}\text { Jackson ImmunoResearch Europe } \\
\text { Ltd., Suffolk, UK }\end{array}$ \\
\hline Cy3 anti-rabbit & $1: 500$ & $\begin{array}{l}\text { Jackson ImmunoResearch Europe } \\
\text { Ltd., Suffolk, UK }\end{array}$ \\
\hline
\end{tabular}


Table 4 Flow cytometric antibodies

\begin{tabular}{|c|c|c|c|c|}
\hline Specificity & Fluorochrome & Clone & Dilution & Source of supply \\
\hline CCR2 & APC & FAB5538A & $1: 100$ & $\begin{array}{l}\text { R\&D Systems Inc., Minneapolis, } \\
\text { Canada }\end{array}$ \\
\hline CD11b & PerCP & $\mathrm{M} 1 / 70$ & $1: 100$ & $\begin{array}{l}\text { BioLegend, San Diego, CA, } \\
\text { USA }\end{array}$ \\
\hline CD19 & FITC & eBio1D3 & $1: 100$ & $\begin{array}{l}\text { eBioscience Ltd., Altrincham, } \\
\text { UK }\end{array}$ \\
\hline CD25 & APC & PC61.5 & $1: 100$ & $\begin{array}{l}\text { eBioscience Ltd., Altrincham, } \\
\text { UK }\end{array}$ \\
\hline CD3 & PE Cy7 & $145-2 C 11$ & $1: 100$ & $\begin{array}{l}\text { eBioscience Ltd., Altrincham, } \\
\text { UK }\end{array}$ \\
\hline CD4 & PerCP & RM4-5 & $1: 100$ & $\begin{array}{l}\text { BD Biosciences, Franklin Lakes, } \\
\text { NJ, USA }\end{array}$ \\
\hline CD45 & APC Cy7 & $30-F 11$ & $1: 100$ & $\begin{array}{l}\text { BioLegend, San Diego, CA, } \\
\text { USA }\end{array}$ \\
\hline CD8 & FITC & $53-6.7$ & $1: 100$ & $\begin{array}{l}\text { BD Biosciences, Franklin Lakes, } \\
\text { NJ, USA }\end{array}$ \\
\hline FoxP3 & PE & FJK-16S & $1: 200$ & $\begin{array}{l}\text { eBioscience Ltd., Altrincham, } \\
\text { UK }\end{array}$ \\
\hline NK1.1 & APC & PK136 & $1: 100$ & $\begin{array}{l}\text { eBioscience Ltd., Altrincham, } \\
\text { UK }\end{array}$ \\
\hline NKp46 & PE & 29A1.4 & $1: 100$ & $\begin{array}{l}\text { BioLegend, San Diego, CA, } \\
\text { USA }\end{array}$ \\
\hline
\end{tabular}

Abbreviations: APC allophycocyanine; CD cluster of differentiation; FITC fluorescein isothiocyanate; PE phycoerythrin; PerCP peridinin chlorophyll protein

Table 5 Proteins and enzymes

\begin{tabular}{l|l}
\hline Proteins and enzymes & Source of supply \\
\hline $\begin{array}{l}\text { alkaline phosphatase - anti alkaline } \\
\text { phosphatase (APAAP) }\end{array}$ & Sigma-Aldrich GmbH, Steinheim, Germany \\
\hline $\begin{array}{l}\text { anti-aquaporin4 antibody (for } \\
\text { stereotactical injection) }\end{array}$ & J. Bennett et al., 2009 (doi:10.1002/ana.21802) \\
\hline Bovine serum albumin (BSA) & $\begin{array}{l}\text { SERVA Electrophoresis GmbH, Heidelberg, } \\
\text { Germany }\end{array}$ \\
\hline
\end{tabular}




\begin{tabular}{|c|c|}
\hline Proteins and enzymes & Source of supply \\
\hline Collagenase D & $\begin{array}{l}\text { Roche Diagnostics Deutschland GmbH, } \\
\text { Mannheim, Germany }\end{array}$ \\
\hline $\begin{array}{l}\text { complement sera from mouse, } \\
\text { lyophilized powder, } 1 \mathrm{U} / \mathrm{ml}\end{array}$ & Sigma-Aldrich GmbH, Steinheim, Germany \\
\hline DNAse I & $\begin{array}{l}\text { Roche Diagnostics Deutschland } \mathrm{GmbH} \text {, } \\
\text { Mannheim, Germany }\end{array}$ \\
\hline Fc-blocking anti-CD16/CD32 antibody & Sigma-Aldrich GmbH, Steinheim, Germany \\
\hline Fetal calf serum (FCS) & Sigma-Aldrich GmbH, Steinheim, Germany \\
\hline Horse serum & Sigma-Aldrich GmbH, Steinheim, Germany \\
\hline Incomplete Freund's Adjuvant (IFA) & Sigma-Aldrich GmbH, Steinheim, Germany \\
\hline Inactive mycobacteria H37RA & BD Biosciences, Franklin Lakes, NJ, USA \\
\hline MOG $_{35-55}$ peptide & $\begin{array}{l}\text { Institute of Medical Immunology, University } \\
\text { Medical Center Charité, Berlin, Germany }\end{array}$ \\
\hline Ovalbumin & Sigma-Aldrich GmbH, Steinheim, Germany \\
\hline Pertussis Toxin (PTX) & $\begin{array}{l}\text { List biological laboratories, Campbell, CA, } \\
\text { USA }\end{array}$ \\
\hline Proteinase $\mathrm{K}$ & Sigma-Aldrich $\mathrm{GmbH}$, Steinheim, Germany \\
\hline Trypsin-EDTA (0.25\%) & $\begin{array}{l}\text { Gibco, Life Technologies GmbH, Darmstadt, } \\
\text { Germany }\end{array}$ \\
\hline $\begin{array}{l}\text { Z2 antibody, } 4.8 \mathrm{mg} / \mathrm{ml} \text { mouse } \lg G 2 \mathrm{~A} \\
\text { (for stereotactical injection) }\end{array}$ & $\begin{array}{l}\text { Hybridoma obtained from C. Linington (C. } \\
\text { Elliot et al., 2012; doi:10.1093/brain/aws105); } \\
\text { purified by S. Nessler }\end{array}$ \\
\hline
\end{tabular}


Table 6 Solutions, buffer and media

\begin{tabular}{|c|c|}
\hline Solution/medium & Composition \\
\hline Anti-MOG antibody solution & $\begin{array}{l}3.6 \mathrm{mg} / \mathrm{ml} \text { Z2 antibody } \\
0.25 \mathrm{U} / \mathrm{ml} \text { mouse complement } \\
\text { add Monastral Blue (approx. 1\% of end } \\
\text { volume) }\end{array}$ \\
\hline Bielschowsky development solution & $\begin{array}{l}20 \mathrm{ml} 37 \% \text { formaldehyde } \\
100 \mathrm{ml} \text { Aqua bidest. } \\
0.5 \mathrm{~g} \text { citric acid } \\
2 \text { drops } 65 \% \text { nitric acid }\end{array}$ \\
\hline $\begin{array}{l}\text { Block mix } \\
\text { (in situ hybridization) }\end{array}$ & $\begin{array}{l}870 \mu \mathrm{l} \mathrm{BBR} \\
100 \mu \mathrm{l} \mathrm{FCS} \\
30 \mu \mathrm{l} \text { human serum }\end{array}$ \\
\hline BME medium & $\begin{array}{l}50 \mathrm{ml} \text { horse serum } \\
5 \mathrm{ml} \text { Penicillin/Streptomycin } \\
5 \mathrm{ml} \text { Glutamax } \\
\text { Dilute in BME medium and filtrate sterile }\end{array}$ \\
\hline CFA (Complete Freunds' Adjuvant) & $\begin{array}{l}\text { Paraffin oil } \\
15 \% \text { mannide monooleate } \\
6.7 \mathrm{mg} / \mathrm{ml} \text { Mycobacterium tuberculosis H37RA }\end{array}$ \\
\hline EM fixation solution (400 ml) & 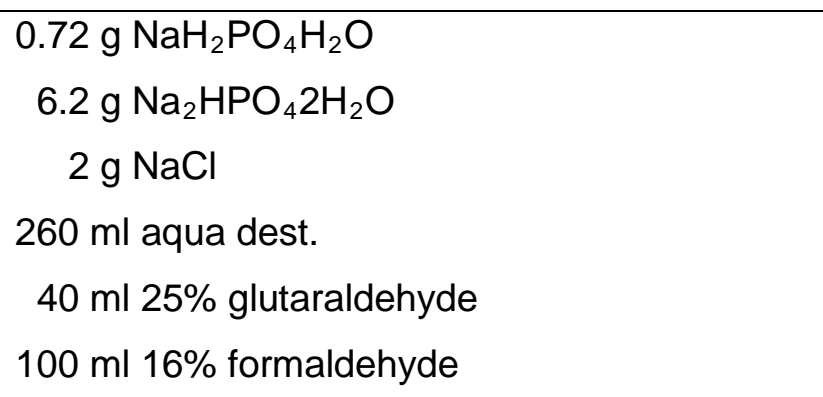 \\
\hline $\begin{array}{l}\text { Hybridization mix Washing buffer I } \\
\text { (in situ hybridization) }\end{array}$ & $\begin{array}{l}10 \mu \mathrm{l} \text { Dextran } \\
5 \mu \mathrm{l} \text { 20x SSC } \\
25 \mu \text { l deionized Formamid } \\
2 \mu \mathrm{l} \text { Salmon-sp.-DNA } \\
0.5 \mu \mathrm{l} \text { 2\% SDS } \\
3 \mu \mathrm{l} \text { PLP probe } \\
5.5 \mu \text { l DPEC- } \mathrm{H}_{2} \mathrm{O}\end{array}$ \\
\hline
\end{tabular}




\begin{tabular}{|c|c|c|}
\hline Solution/medium & \multicolumn{2}{|c|}{ Composition } \\
\hline Ketamin/Xylacin anesthesia & \multicolumn{2}{|c|}{$\begin{array}{l}\mathrm{NaCl}, 0.9 \%, \text { sterile } \\
12 \% \text { ketamine } \\
10 \% \text { xylazine }\end{array}$} \\
\hline LFB solution & \multicolumn{2}{|c|}{$\begin{array}{l}1 \mathrm{~g} \mathrm{LFB} \\
1 \text { I } 96 \% \text { ethanol } \\
5 \mathrm{ml} 10 \% \text { acetic acid }\end{array}$} \\
\hline $\begin{array}{l}\text { NBT/BCIP substrate buffer } \\
\text { (in situ hybridization) }\end{array}$ & \multicolumn{2}{|c|}{$\begin{array}{l}175 \mu \mathrm{l} \text { NBT } \\
225 \mu \mathrm{l} \mathrm{BCIP} \\
\text { fill up with substrate buffer to } 50 \mathrm{ml}\end{array}$} \\
\hline Paraformaldehyde (PFA, 4\%) & \multicolumn{2}{|c|}{$\begin{array}{l}\text { PBS } \\
\text { 4\% PFA } \\
\text { adjust } \mathrm{pH} \text { to } 7.4\end{array}$} \\
\hline PLP development solution & I. & $\begin{array}{l}\text { Dilute } 10 \mathrm{mg} \text { sodium nitrate in } 250 \\
\mu \mathrm{l} \text { aqua bidest. } \\
\text { Dilute } 14 \mathrm{mg} \text { Naphthol As-Bi } \\
\text { phosphate in } 300 \mu \mathrm{NN}- \\
\text { dimethylformamide. } \\
\text { Dilute } 20 \mathrm{mg} \text { Levamisol in } 50 \mathrm{ml} \\
\text { TBS, add I. and II. and adjust to pH } \\
8.8\end{array}$ \\
\hline Proteinase $\mathrm{K}$ solution & $\begin{array}{l}34.2 \mathrm{n} \\
800 \mu \mathrm{l} \\
375 \mu \mathrm{l}\end{array}$ & $\begin{array}{l}\mathrm{BS} \\
\mathrm{Cl}_{2} \\
\text { teinase } \mathrm{K}\end{array}$ \\
\hline Super SATO medium & $\begin{array}{l}2 \mathrm{ml} \mathrm{B} \\
1 \mathrm{ml} \mathrm{G} \\
1 \mathrm{ml} \mathrm{P} \\
1 \mathrm{ml} \mathrm{h} \\
1 \mathrm{ml} \mathrm{s} \\
10 \mu \mathrm{t} \\
96 \% \mathrm{e} \\
13 \mu \mathrm{l} \\
\mathrm{NaOH} \\
\text { Fill up } \\
\text { Glutar }\end{array}$ & $\begin{array}{l}\text { supplement } \\
\text { amax } \\
\text { cillin/Streptomycin } \\
\text { serum } \\
\text { Im pyruvate solution } 1.1 \% \\
\text { dothyronine solution ( } 0.034 \mathrm{~g} \text { in } 10 \mathrm{ml} \\
\text { nol) } \\
\text { yyroxin solution ( } 0.062 \mathrm{~g} \text { in } 0.104 \mathrm{~g} \\
\mathrm{nl} \mathrm{H}_{2} 0,14 \mathrm{ml} 70 \% \text { ethanol) } \\
\text { DMEM (+4.5g/l D-glucose, }+\mathrm{L}- \\
\text { - }- \text { Pyruvate) and filtrate sterile }\end{array}$ \\
\hline
\end{tabular}




\begin{tabular}{l|l}
\hline Solution/medium & Composition \\
\hline & $7.5 \mathrm{ml} 20 \times \mathrm{SSC}$ \\
Washing buffer I (in situ hybridization) & $1.5 \mathrm{ml} 10 \%$ SDS \\
& fill up with DEPC- $\mathrm{H}_{2} \mathrm{O}$ to $150 \mathrm{ml}$ \\
\hline $\begin{array}{l}\text { Washing buffer II Washing buffer I } \\
\text { (in situ hybridization) }\end{array}$ & $1 \mathrm{ml} 20 \times \mathrm{SSC}$ \\
& $1 \mathrm{ml} 10 \% \mathrm{SDS}$ \\
\hline
\end{tabular}

Table 7 Consumables

\begin{tabular}{|c|c|}
\hline Consumable & Source of supply \\
\hline Bottle top filter, $0.2 \mu \mathrm{m}$ & Sarstedt, Nümbrecht, Germany \\
\hline Blaubrand $^{\circledR}$ intraMARK micropipettes, $5 \mu \mathrm{l}$ & Brand GmbH, Wertheim, Germany \\
\hline Cell culture flask, $75 \mathrm{~cm}^{2}$, Falcon & TH Geyer, Hamburg, Germany \\
\hline $\begin{array}{l}\text { Cell culture plate, flat bottom ( } 24 \text { well, } \\
96 \text { well) }\end{array}$ & Greiner bio-one, Kremsmünster, Austria \\
\hline Cell strainer (40 $\mu \mathrm{m})$ & BD Biosciences, Franklin Lakes, NJ, USA \\
\hline FACS tube, $5 \mathrm{ml}$ & BD Biosciences, Franklin Lakes, NJ, USA \\
\hline $\begin{array}{l}\text { Nunc }^{\mathrm{TM}} \text { Microwell } \\
\text { bottom }\end{array}$ & $\begin{array}{l}\text { Thermo Fisher Scientific, Waltham, MA, } \\
\text { USA }\end{array}$ \\
\hline Petri dishes $(3 \mathrm{~cm}, 7 \mathrm{~cm} \varnothing)$ & Greiner bio-one, Kremsmünster, Austria \\
\hline Serological pipettes (5 ml, $10 \mathrm{ml}, 25 \mathrm{ml}$ ) & Sarstedt, Nümbrecht, Germany \\
\hline Signa Gel ${ }^{\circledR}$ electrode conductive gel & Parker Laboratories Inc., Fairfield, NJ, USA \\
\hline Syringes & BD Biosciences, Franklin Lakes, NJ, USA \\
\hline Ten20 & Weaver and Company, Aurora, CO, USA \\
\hline $\begin{array}{l}\text { Tubes }(50 \mathrm{ml}, 15 \mathrm{ml}, 10 \mathrm{ml}, 2 \mathrm{ml}, 1.5 \mathrm{ml} \\
0.5 \mathrm{ml})\end{array}$ & Sarstedt, Nümbrecht, Germany \\
\hline
\end{tabular}


Table 8 Technical devices

\begin{tabular}{|c|c|}
\hline Technical device & Source of supply \\
\hline 5810R centrifuge & Eppendorf, Hamburg, Germany \\
\hline CCD Camera & MegaView III, Soft Imaging System $®)$ \\
\hline C-Dish ${ }^{\circledR}$ & IonOptix, Rathmines, Ireland \\
\hline $\begin{array}{l}\text { DS5 Isolated Bipolar Constant Current } \\
\text { Stimulator }\end{array}$ & Digitimer, Welwyn Garden City, UK \\
\hline EM10B electron microscope & $\begin{array}{l}\text { Carl Zeiss Microscopy GmbH, Oberkochen, } \\
\text { Germany }\end{array}$ \\
\hline FACS Canto ${ }^{\mathrm{TM}}$ II & BD Bioscience, Franklin Lakes, NJ, USA \\
\hline Heracell VIOS 160i incubator & $\begin{array}{l}\text { Thermo Fisher Scientific, Waltham, MA, } \\
\text { USA }\end{array}$ \\
\hline Sigma Delta Vaporizer ${ }^{\circledR}$ (Isoflurane pump) & Penton Limited, Abingdon, UK \\
\hline Isolated Pulse Stimulater 2100 & A-M Systems, Carlsborg, USA \\
\hline Leica Ultracut & Leica, Wetzlar, German \\
\hline Magstim 200 magnetic stimulator & Magstim, Whiteland, Dyfed, UK \\
\hline Microtome & Leica, Wetzlar, German \\
\hline Microwave & Bosch, Gerling-Schillerhohe, Germany \\
\hline Neubauer counting chamber & $\begin{array}{l}\text { Superior Marienfeld, Lauda-Königshofen, } \\
\text { Germany }\end{array}$ \\
\hline Olympus BX51 fluorescence microscope & Olympus Corporation, Tokyo, Japan \\
\hline $\begin{array}{lll}\text { Olympus } & \text { VS120 fluorescence } & \text { slide } \\
\text { scanner } & & \\
\end{array}$ & Olympus Corporation, Tokyo, Japan \\
\hline Power 1401 AD converter & $\begin{array}{l}\text { Cambridge Electronic Design, Cambridge, } \\
\text { UK }\end{array}$ \\
\hline Stereotactic device & Stoelting Co, Wood Dale, IL, USA \\
\hline Tecan Safire plate reader & Tecan Group Ltd., Männedorf, Switzerland \\
\hline
\end{tabular}


Table 9 Software

\begin{tabular}{l|l|l}
\hline Software & Application & Source of supply \\
\hline $\begin{array}{l}\text { BD FACSDiva } \\
\text { Software v6.1.2 }\end{array}$ & Data acquisition flow cytometry & $\begin{array}{l}\text { BD Biosciences, Franklin } \\
\text { Lakes, NJ, USA }\end{array}$ \\
\hline FlowJo v7.6.1 & Data analysis flow cytometry & $\begin{array}{l}\text { FlowJo LLC, Oregon, } \\
\text { U.S. }\end{array}$ \\
\hline GraphPad Prism v5.0 & Statistical analysis & $\begin{array}{l}\text { GraphPad software Inc., La } \\
\text { Jolla, CA, USA }\end{array}$ \\
\hline ImageJ v1.50b & Image processing and analysis & $\begin{array}{l}\text { ImageJ, National Institute of } \\
\text { Health, USA }\end{array}$ \\
\hline $\begin{array}{l}\text { Olympus cellsense } \\
\text { dimension v1.7.1 }\end{array}$ & Image acquisition ESTIM in vitro & $\begin{array}{l}\text { Olympus } \\
\text { Tokyo, Japan }\end{array}$ \\
\hline Olympus Olyvia v2.9 & Whole slide image evaluation & $\begin{array}{l}\text { Olympus Corporation, } \\
\text { Tokyo, Japan }\end{array}$ \\
\hline Olympus VS-ASW v2.9 & Image acquisition ESTIM in vivo & $\begin{array}{l}\text { Olympus } \\
\text { Tokyo, Japan }\end{array}$ \\
\hline R v3.1.2 & Data analysis & $\begin{array}{l}\text { The R Foundation for } \\
\text { Statistical Computing }\end{array}$ \\
\hline Signal v2.16 & $\begin{array}{l}\text { Software for eTBS script and } \\
\text { MEP recording }\end{array}$ & $\begin{array}{l}\text { Cambridge Electronic } \\
\text { Design Ltd. }\end{array}$ \\
\hline
\end{tabular}




\section{Part I - Methods}

Influence of autoimmune inflammation on remyelination in Cuprizone-induced demyelination 


\subsection{Cuprizone-EAE}

\subsubsection{Animals}

A total number of 77 adult wildtype C57BL/6J mice (Charles River) aged between six and eight weeks were held in groups of maximal eight animals per cage with food and water ad libitum and on a 12:12 h light/dark cycle. The animals were separated into four groups: cuprizone treated animals (Cup, $n=29$ ), cuprizone treated animals immunized with $\mathrm{MOG}_{35-55}$ peptide (Cup-EAE, $n=32$ ), cuprizone treated animals which were injected with ovalbumin instead of $\mathrm{MOG}_{35-55}$ peptide (Cup-Ova, $\mathrm{n}=12$ ) and untreated control animals (control, $\mathrm{n}=4$ ). All animal experiments were carried out in accordance to the Bundesamt für Verbraucherschutz Oldenburg, Germany.

\subsubsection{Cuprizone-induced demyelination}

To induce demyelination (DM) of the corpus callosum (CC) mice were fed with a $0.25 \%$ Cuprizone diet the first two weeks. Afterwards, the dose was decreased to $0.15 \%$ Cuprizone for the remaining three weeks. Cuprizone is a copper chelator which leads to a toxic demyelination, most prominent in the corpus callosum, and was administered by mixing cuprizone powder with normal mill chow. Non-treated control animals were fed with normal chow for the same time period. A detailed overview of the experimental time schedule is shown in Figure 3.

\subsubsection{Induction of brain inflammation}

To induce an infiltration of immune competent cells into the CNS, animals in the CupEAE group received a $200 \mu \mathrm{l}$ emulsion, separated into four $50 \mu \mathrm{l}$ reservoirs and injected periaxillary and peri-inguinally. The emulsion consisted of $1 \mathrm{ml}$ complete Freund's adjuvants (CFA; incomplete Freund's adjuvants with inactive mycobacteria H37RA) mixed evenly with $1 \mathrm{mg} / \mathrm{ml} \mathrm{MOG}_{35-55}$ peptide. The emulsion used for the ovalbumin control group was prepared by the same protocol, however MOG $_{35-55}$ peptide was replaced by the CNS-unspecific antigen ovalbumin. The mice were immunized at day 17 and once again at day 24 after starting cuprizone treatment (Fig. 3). To enhance the reaction of the immune system, $300 \mathrm{ng}$ Pertussis Toxin (PTX) was injected into Cup-EAE and Cup-Ova animals at the day of the first immunization, as well as 2 and 8 days later. Neurological signs were assessed as described in table 10. If animals reached a score of 3.5 or lost more than $25 \%$ of their weight, they were sacrificed prematurely to prevent unnecessary animal suffering. 


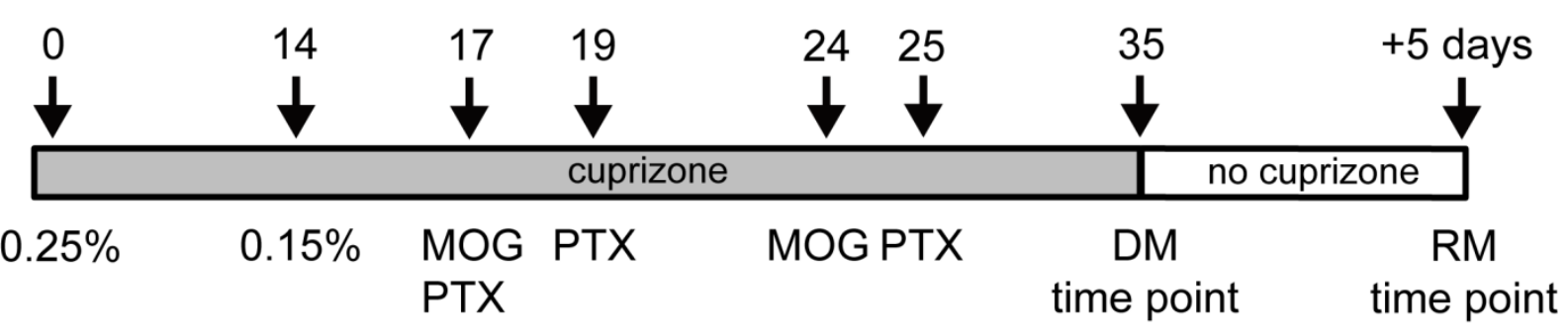

Figure 3: Time scale of cuprizone treatment and EAE induction

Mice were fed cuprizone for 35 days. The initial dose of $0.25 \%$ cuprizone was reduced to $0.15 \%$ after 14 days, to allow $T$ cells to infiltrate the CNS without severe impairment. MOG $_{35-55}$ peptide was injected twice in mice from the Cup-EAE group, first after 17 days and the second time after 24 days. Subsequently, PTX was injected in the same mice, to amplify the immune reaction. In Cup-Ova animals, ovalbumin was injected instead of $\mathrm{MOG}_{35-55}$ peptide. All mice from the Cup-Ova group as well as half of the mice from the Cup and Cup-EAE groups were sacrificed after 35 days to establish the maximal extent of DM, while the remaining animals were sacrificed after five additional days on a cuprizone-free diet to investigate the extent of RM. The time points of immunization with MOG $_{35-55}$ peptide were adopted from the doctoral thesis of Angelika Escher, 2008.

Table 10: EAE score assessment

\begin{tabular}{|c|c|}
\hline score & clinical symptoms \\
\hline 0 & no symptoms \\
\hline 0.5 & partially limb tail \\
\hline 1 & limb tail \\
\hline 1.5 & $\begin{array}{l}\text { limb tail + slight } \\
\text { paresis of hind legs }\end{array}$ \\
\hline 2 & $\begin{array}{l}\text { limb tail + severe } \\
\text { paresis of hind legs }\end{array}$ \\
\hline 2.5 & $\begin{array}{c}\text { severe paresis of hind } \\
\text { legs + one leg } \\
\text { dragged behind }\end{array}$ \\
\hline 3 & paralyzed hind legs \\
\hline 3.5 & $\begin{array}{l}\text { paralyzed hind legs + } \\
\text { slight paresis of } \\
\text { forelegs }\end{array}$ \\
\hline
\end{tabular}

\subsubsection{Electron microscopy}

To examine the density of myelinated axons in the corpus callosum (CC), electron microscopic pictures were taken using an EM 10 from ZEISS and analyzed. For this purpose, the animals were anaesthetized by injecting an overdose of $14 \%$ chloral hydrate and perfused transcardially with an EM fixation solution. In contrast to (immuno-) histochemical stainings, sagittal sections of the brains were prepared. After dissection of the brains, pieces of the CC were washed with $0.1 \mathrm{M}$ phosphate buffered saline (PBS), fixed with $3 \%$ glutaraldehyde, post fixed and contrasted with 1\% osmium tetroxide followed by dehydration through an ascending ethanol series (50\% ethanol for $10 \mathrm{~min}, 0.5 \%$ uranyl acetate in 70\% 
ethanol for $1 \mathrm{~h}$ at $4^{\circ} \mathrm{C}, 80 \%$ ethanol for $10 \mathrm{~min}, 96 \%$ ethanol for $10 \mathrm{~min}, 100 \%$ ethanol twice for $15 \mathrm{~min}$, propylene oxide twice for $20 \mathrm{~min}$ ). Subsequently, the samples were embedded in Renlam M1 by incubation in a 1:1 mixture of Renlam M1 and propylene oxide for 35 min followed by a 2:1 mixture of Renlam M1 and propylene oxide for $35 \mathrm{~min}$ and pure Renlam M1 for $40 \mathrm{~min}$. Semi-thin sections (360 nm) were cut and stained with toluidine blue to define the region of interest in the $\mathrm{CC}$. Accordingly, ultra-thin sections $(72 \mathrm{~nm})$ of the region of interest were cut and contrasted with lead citrate for $10 \mathrm{~min}$. For analysis of axonal diameter and myelin thickness, a magnification of x12000 was used. Quantitative analysis of myelinated axons was carried out using ImageJ and R.

\subsubsection{Histopathology}

\subsubsection{Histochemical stainings}

Animals were anaesthetized by injecting an overdose of $14 \%$ chloral hydrate and transcardially perfused with $0.1 \mathrm{M}$ PBS followed by $4 \%$ paraformaldehyde. Brains were removed and post fixed in $4 \%$ paraformaldehyde for two days at $4{ }^{\circ} \mathrm{C}$. Afterwards, brains were dissected and cut into 3-5 mm thick coronal slices, which were dehydrated in an ascending ethanol series before being embedded in paraffin. Brain slices were cut with 2-4 $\mu \mathrm{m}$ width and stained with Luxol fast blue / periodic acid Schiff (LFB/PAS) for detection of myelin, as described in the following section.

\section{Luxol fast blue I periodic acid Schiff (LFB/PAS) staining}

If not indicated otherwise, the following steps were performed at room temperature. In preparation for the LFB/PAS staining, the brain slices were placed on microscope slides, deparaffinized (three times $3 \mathrm{~min}$ in xylol and $3 \mathrm{~min}$ in isoxylol) and rehydrated ( $3 \mathrm{~min}$ in ethanol $100 \%$, followed by ethanol $90 \%$ ). Afterwards, the slides were placed in a LFB solution overnight at $60^{\circ} \mathrm{C}$.

On the next day, the slides were put in $90 \%$ ethanol for $5 \mathrm{~min}$, followed by differentiation steps in $0.05 \%$ lithium carbonate and $70 \%$ ethanol and washed with aqua dest. For the second part of the LFB/PAS staining, the periodic acid Schiff, the brain slices were placed in 1\% periodic acid, washed thoroughly in tap water and processed in Schiff's solution for $20 \mathrm{~min}$, followed by another washing step in tap water to stain the parenchyma. To stain the cell nuclei, the slides were placed for 2 min in Mayer's hemalum solution and, after another washing step, differentiated in $1 \% \mathrm{HCl}$, followed by treatment with tap water and an ascending ethanol series which finished xylol. The determination of the demyelinated area was done using the semi-quantitative score shown in table 11 (DM score originally established by Hiremath et al., 1998). 
Table 11: Demyelination score assessment

\begin{tabular}{cc} 
score & $\begin{array}{c}\text { Degree of demyelination in the } \\
\text { corpus callosum }\end{array}$ \\
\hline 0 & no loss of myelin \\
1 & less than $1 / 3$ demyelinated \\
2 & $1 / 3-2 / 3$ demyelinated \\
3 & more than $2 / 3$ demyelinated
\end{tabular}

\subsubsection{Immunohistochemical stainings}

All steps were conducted in a dark and moist chamber to prevent bleaching and dehydration of the brain slices. By tissue fixation and embedding in paraffin, a masking of the antigens can occur. Since an efficient binding of the primary antibody and the target antigen is required for a successful immunostaining, a pretreatment might be necessary to unmask the antigens, before conducting any further steps. A complete list of all primary antibodies as well as the necessary pretreatments and the respective secondary antibodies can be found in table 2 and table 3 . If not indicated otherwise $50 \mu$ of the respective solution was used on each object slide, and all incubations were performed at room temperature, except if incubated overnight, which were performed at $4{ }^{\circ} \mathrm{C}$.

\section{Olig2 and NogoA}

Immunohistochemical stainings with primary antibodies against the transcription factor Olig2 and the neurite growth inhibitor NogoA were used for detection of oligodendrocyte lineage cells and mature oligodendrocytes, respectively. Both antibodies required a pretreatment with citrate buffer, $\mathrm{pH} 6.0,10 \mathrm{mM}$, to unmask the antigens. For this purpose, the brain slices were placed in citrate buffer in a microwave oven and heated for 3 min. Afterwards, the citrate buffer was exchanged by PBS and the slices were placed in the microwave oven for another $3 \mathrm{~min}$. This process was repeated two more times and the slices were then washed thoroughly with PBS. To block the endogenous peroxidase of the tissue and the unspecific binding sites, all slides were processed in $50 \mathrm{ml} 3.33 \% \mathrm{H}_{2} \mathrm{O}_{2}$ in PBS, followed by a PBS solution with $10 \%$ fetal calf serum (FCS; 10 min at $4^{\circ} \mathrm{C}$ ). Each primary antibody was diluted in a 10\% FCS/PBS solution (Olig2: 1:300; NogoA: 1:15000), $100 \mu \mathrm{l}$ of which were equally applied onto each slide (for a complete list of primary antibodies see table 2). The incubation was carried out at $4 \mathrm{C}^{\circ}$ overnight. On the next day, all slides were washed with PBS and incubated with the secondary antibody (biotinylated anti-rabbit 1:200, in 10\% FCS/PBS; table 3) for 1 hour. Finally, all brain slices were incubated with peroxidaseconjugated streptavidin (1:1000 in PBS), processed with the chromogen amino ethyl carbazol (AEC) to visualize the antibodies and embedded with Immu-Mount mounting medium. 


\section{Amyloid precursor protein (APP)}

To identify axonal damage, an APP primary antibody was used. The slides received the same treatment as described for the Olig2 and NogoA stainings as well as the same pretreatment with citrate buffer. To visualize the antibody, the chromogen 3,3'-Diaminobenzidine (DAB) was used instead of AEC. To mark the cell nuclei, the slides were incubated for 10 sec in Mayer's hemalum solution, differentiated in $1 \% \mathrm{HCl}$ solution and treated with tap water, followed by an ascending ethanol series.

\section{Proteolipid protein (PLP)}

A PLP immunohistochemical staining combined with an in situ hybridization was performed to label the PLP protein (table 2) and the PLP mRNA, respectively. The in situ hybridization was performed as described by Breitschopf et al. in 1992. Therefore, all slides were treated twice with xylol for 20 min each to remove the paraffin. A descending ethanol series (96\%, 96\%, 75\%, 50\%, 30\%, DEPC- $\mathrm{H}_{2} \mathrm{O}, 3 \mathrm{~min}$ each) was performed to rehydrate the tissue, followed by a post-fixation with $4 \%$ PFA for 20 min at $4{ }^{\circ} \mathrm{C}$. After a washing step with TBS (tris-buffered saline) the slides were treated with $0.2 \mathrm{M} \mathrm{HCl}$ for 10 min to denature the proteins of the tissue, followed by another washing step with TBS. To expose the mRNA the proteins had to be digested. This was done by processing the slides with $50 \mu \mathrm{g} / \mathrm{ml}$ proteinase $\mathrm{K}\left(34.2 \mathrm{ml} \mathrm{TBS}+800 \mu \mathrm{CaCl}_{2}+375 \mu \mathrm{l}\right.$ proteinase $\left.\mathrm{K}\right)$ for $20 \mathrm{~min}$ at $37^{\circ} \mathrm{C}$. Afterwards, the slices were treated with cold TBS $\left(-20^{\circ} \mathrm{C}\right)$ for $5 \mathrm{~min}$ to stop the digestion. To inactivate the endogenous alkaline phosphatase, all brain slices were treated with $0.5 \%$ acetic anhydride (45 ml $0.1 \mathrm{M} \mathrm{TBS}+225 \mu \mathrm{l}$ acetic anhydride) for $10 \mathrm{~min}$. After another washing step and an ascending ethanol series $(30 \%, 50 \%, 75 \%, 96 \%, 96 \%$, chloroform, 3 min each) for dehydration, the slides had to dry at room temperature. For the hybridization with the PLP probe $50 \mu \mathrm{l}$ of the hybridization mix was administered to each slice which were then covered with a cover slip and incubated for $4 \mathrm{~min}$ at $95{ }^{\circ} \mathrm{C}$ to disintegrate the secondary structure of the RNA, thus allowing an easier binding of the probe. Afterwards, the samples were placed overnight in a moist chamber at $62{ }^{\circ} \mathrm{C}$. The next day, all slides were treated with washing buffer I two times for 10 min each, followed by two times 10 min with washing buffer II at 62 ${ }^{\circ} \mathrm{C}$ and two times 10 min with TBS at room temperature. In preparation for the antibody incubation with anti-DigoxigeninAP, the slides were incubated with $100 \mu \mathrm{l}$ of block solution for 15 min in a moist chamber. The anti-DigoxigeninAP antibody was diluted 1:250 in block solution and incubated on the slices for 2 hours in a moist chamber. Afterwards, the slides were washed three times with TBS and once with 4-nitro blue tetrazolium chloride / 5-bromo4-chloro-3-indolyl phosphate (NBT/BCIP) substrate buffer, followed by a final incubation period overnight at $4{ }^{\circ} \mathrm{C}$ in NBT/BCIP substrate buffer. 
In preparation for the immunohistochemical staining, all slices were incubated in $10 \%$ FCS/TBS for 10 min to block unspecific binding sites. Subsequently, the primary antibody (PLP, mouse, 1:500) was incubated for one hour, followed by a washing step with TBS and the secondary antibody (rabbit anti-mouse, 1:50, incubation time one hour). The secondary antibody connects the primary antibody with the alkaline phosphatase - anti alkaline phosphatase (APAAP) complex (1:50, incubation time one hour), which can be visualized by a specific development solution, followed by treatment in Mayer's hemalum solution for 30 seconds.

\subsubsection{Flow cytometric analysis}

Animals used for flow cytometric (FACS) analysis were anaesthetized by injecting an overdose of $14 \%$ chloral hydrate and transcardially perfused with $0.1 \mathrm{M}$ PBS. Brains were dissected, disintegrated, centrifuged for $12 \mathrm{~min}$ at $1200 \mathrm{rpm}$, resuspended in $800 \mu \mathrm{l}$ DMEM (without FCS) and digested with $1 \mathrm{mg} / \mathrm{ml}$ DNAse and $2.5 \mathrm{mg} / \mathrm{ml}$ collagenase for $45 \mathrm{~min}$. The digestion was stopped with DMEM (+2\% FCS), the samples were centrifuged for 7 min at $1200 \mathrm{rpm}$ and the pellets were resuspended in $5 \mathrm{ml}$ DMEM+FCS. Subsequently, the cells were separated from myelin by a Percoll gradient. For this purpose, a $37 \%$ and a $70 \%$ Percoll phase was created and the cell solution was added carefully. The cells were separated from myelin by centrifugation at $1600 \mathrm{rpm}$ for $25 \mathrm{~min}$ after which they were extracted and transferred to PBS ( $+2 \%$ FCS). In order to label the cells with FACS antibodies, they were transferred to round bottom 96 well plates, which were centrifuged for $2 \mathrm{~min}$ at $1200 \mathrm{rpm}$, followed by a treatment with Fc-blocking anti-CD16/CD32 antibody (1:100) for $10 \mathrm{~min}$ at $4^{\circ} \mathrm{C}$. Subsequently, a mixture of FACS antibodies (table 4 , table 11 ) was added for $10 \mathrm{~min}$ at $4^{\circ} \mathrm{C}$. For intracellular stainings like FoxP3, cells were fixed and permeabilized before the antibody incubation to ensure an optimal labeling. Therefore, the cells were washed twice in PBS $+2 \%$ FCS before they were fixed for 45 min in FoxP3 fixation concentrate (1:4 in FoxP3 fixation diluent). Afterwards, the cells were washed twice in FoxP3 permeabilization buffer and labeled with FoxP3 (1:200 in permeabilization buffer) for $30 \mathrm{~min}$. All labeled cells were washed twice in PBS+ $2 \%$ FCS before being measured using a BD FACSCanto II flow cytometer. Quantitative analysis was performed using FlowJo version 7.6.1. 
Table 12: Classification of leukocyte subpopulations

\begin{tabular}{|c|c|}
\hline Leukocyte & Specific surface and intracellular marker \\
\hline T cells & $\mathrm{CD}_{4} 5^{+} \mathrm{CD}^{+} \mathrm{CD}^{-} 9^{-}$ \\
\hline NK cells & $\mathrm{CD} 45^{+} \mathrm{CD}^{-} \mathrm{CD}^{-} 9^{-} \mathrm{NK} 1^{+} 1^{+} \mathrm{NKp} 6^{+}$ \\
\hline B cells & $\mathrm{CD}^{4} 5^{+} \mathrm{CD}^{-} \mathrm{CD} 19^{+}$ \\
\hline Granulocytes & $\mathrm{CD}_{4} 5^{+}$CD11b ${ }^{+}$Ly6G $^{+}$ \\
\hline Inflammatory monocytes & $\mathrm{CD} 45^{+} \mathrm{CD}_{11 b^{+} \text {Ly6C }^{+} \text {Ly6G }^{-} \text {CCR2 }}{ }^{+}$ \\
\hline Microglia & $\mathrm{CD}^{\mathrm{int}} \mathrm{CD}^{\text {in }} 1 \mathrm{~b}^{\text {int }}$ Ly6C' Ly6C- \\
\hline
\end{tabular}

\subsubsection{Statistical analysis}

The statistical analysis was performed using GraphPad Prism for Windows version 5.0.0. t-Tests were used to compare two experimental groups with each other. In case of non-Gaussian distribution, a Mann-Whitney test was performed. For the analysis of flow cytometric data, one-way ANOVA followed by Bonferroni's Multiple Comparison Test was performed. If the data did not follow Gaussian distribution, a Kruskal-Wallis test was performed, followed by Dunn's Multiple Comparison Test. To compare more than two groups, Two-way ANOVA followed by Sidak's multiple comparisons test was performed. Repeated measures Two-Way ANOVA followed by Bonferroni's Multiple Comparison Test was performed to compare the density of myelinated axons with the parameters COND (condition) for Cup, Cup-EAE and naïve animals and TIME for the DM and RM time points. A $p$ value of $\leq 0.05$ was considered significant and a $p$ value between $0.1-0.051$ was considered a trend. Data are presented as mean \pm SEM. 


\section{Part II - Methods}

Influence of transcranial alternating current stimulation on spontaneous remyelination in in vitro and in vivo models 


\subsection{Electrical stimulation in mixed cortical cultures}

To evaluate the effect of electrical stimulation on the viability and myelin expression of oligodendrocytes, primary cell cultures of rat oligodendrocytes with and without cortical neurons were established.

\subsubsection{Primary cultures - oligodendrocytes}

Two days prior to the isolation of oligodendrocytes, five cell culture flasks $\left(75 \mathrm{~cm}^{2}\right)$ were coated with $0.01 \%$ poly-L-lysin (PLL) overnight at $37^{\circ} \mathrm{C}$ to enhance adherence of oligodendrocytes. On the next day, the cell culture flasks were washed twice with PBS, equipped with $\mathrm{BME}$ medium and placed in an incubator at $37^{\circ} \mathrm{C}$ and $5 \% \mathrm{CO}_{2}$ overnight.

6 newborn Wistar rats from the in-house breeding facility were used on postnatal day 0 (P0) for each experiment (a total of 60 Wistar rats was used) to create primary oligodendrocyte cultures. The following steps were performed using sterile scissors and forceps which were frequently cleaned in aqua dest. and sterilized in $70 \%$ ethanol. The animals were decapitated and the head was placed in a petri dish filled with Hank's balanced salt solution (HBSS) at $37^{\circ} \mathrm{C}$. A cut was made along the medial line of the skull and two more under each ear to provide access to the brain which was removed by using a blunt spatula. After separating the hemispheres and removing the cerebellum, the meninges and the choroid plexus, the brains were digested with $5 \mathrm{ml}$ of $0.25 \%$ Trypsin-EDTA at $37^{\circ} \mathrm{C}$ for 20 min in a $50 \mathrm{ml}$ Falcon tube. Afterwards, the brains were washed once with HBSS and $10 \mathrm{ml}$ BME medium $\left(37^{\circ} \mathrm{C}\right)$ was added. To homogenize the brains, they were pipetted up and down 20 times and centrifuged for $8 \mathrm{~min}$ at $800 \mathrm{rpm}$. The pellet was resuspended in $10 \mathrm{ml} \mathrm{BME}$ medium and the cell suspension was equally distributed between the five cell culture flasks, coated with PLL. The cells were allowed to grow until confluent (approx. 10 days) with medium changes every 3 days.

Once the cells were grown fully confluent, the oligodendrocytes were transferred onto $1 \mathrm{~cm}^{2}$ coverslips placed in $3 \mathrm{~cm}$ petri dishes (each petri dish holds two coverslips). The coverslips were previously coated with PLL (overnight at $37^{\circ} \mathrm{C}$ ) and incubated with SuperSATO medium (overnight at $37^{\circ} \mathrm{C}$ ). To prevent the coverslips from slipping out of place inside the petri dishes, a "frame" was carved around each coverslip using a scalpel.

In order to isolate oligodendrocytes from the mixed cultures containing astrocytes, oligodendrocytes and microglia, first the microglia were shaken off by knocking the cell culture flasks firmly. Afterwards, the flasks were washed once with BME medium, supplied with new BME medium and vigorously shaken 20 times in a horizontal fashion to shake off the oligodendrocytes from the astrocyte layer. The cell suspension was transferred through a $40 \mu \mathrm{m}$ mesh into a $50 \mathrm{ml}$ tube. After $10 \mathrm{~min}$ of centrifugation at $800 \mathrm{rpm}$, the pellet was resuspended in $2 \mathrm{ml}$ Super-SATO medium and transferred in a $3.5 \mathrm{~cm}$ petri dish (uncoated). 
Since microglia tend to attach to the plastic surface of the petri dish, this method was used to further separate the oligodendrocytes from microglia. After 30 seconds, the cell suspension was transferred to a $5 \mathrm{ml}$ tube and the amount of cells in the suspension was determined using a Neubauer counting chamber. For this purpose, $1 \mu \mathrm{l}$ of the cell suspension was mixed with $9 \mu \mathrm{l}$ of tryptophan blue (1:10) to mark apoptotic cells. Cells were transferred into petri dishes equipped with coverslips at a density of 250000 cells per petri dish and separated into an electrical stimulation group (ESTIM) and a non-stimulated control group (no ESTIM). The electrical stimulation was performed one day after the transfer (Fig. $4 \mathrm{~A}$ ). Half the medium of each petri dish was exchanged by fresh Super-SATO medium each day.

\subsubsection{Primary cultures - oligodendrocytes and neurons}

To create oligodendrocyte/neuron cocultures, initially neurons were grown separately on $1 \mathrm{~cm}$ coverslips, previously coated with PLL and placed in $3 \mathrm{~cm}$ petri dishes, for two weeks. For this purpose, prenatal rats with an embryonic age of day 18 (E18) were obtained from a pregnant Wistar rat from the in-house breeding facility (a total of 6 pregnant Wistar rats were used). The following steps were performed using sterile scissors and forceps which were frequently cleaned in aqua dest. and sterilized in $70 \%$ ethanol.

The rat was killed with $\mathrm{CO}_{2}$ and the amniotic sacs were removed. After detaching the amniotic sac, the E18 rats were decapitated and the brains were removed and freed of meninges using forceps only. The brains were digested with 0.25\% Trypsin-EDTA and washed once with BME medium. The brains were homogenized by pipetting them up and down 20 times and subsequently centrifuged for $8 \mathrm{~min}$ at $800 \mathrm{rpm}$. The pellet was resuspended in $10 \mathrm{ml}$ BME medium and the cell count was determined by a Neubauer counting chamber. Each previously prepared $3 \mathrm{~cm}$ petri dish received 500000 cells. The cells were placed in an incubator at $37^{\circ} \mathrm{C}$ and $5 \% \mathrm{CO}_{2}$ for two weeks. The Super-SATO medium was changed every 2-3 days.

In parallel, oligodendrocyte cultures were created as described in 2.3.1 and cultured for six days, before transferred into the petri dishes with the neuronal cultures at day 14 , respectively (a schematic display of the timeline can be found at Fig. 4 B). The oligodendrocyte/neuron cocultures were separated into two experimental groups: an electrical stimulation group (ESTIM) and a non-stimulated control group (no ESTIM). Both groups were cultivated for another day, before the start of the electrical stimulation. Half the medium of each petri dish was exchanged by fresh Super-SATO medium each day. 
A

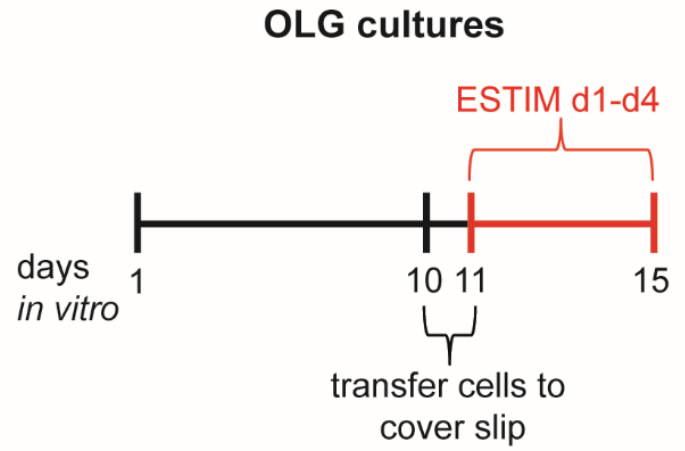

B

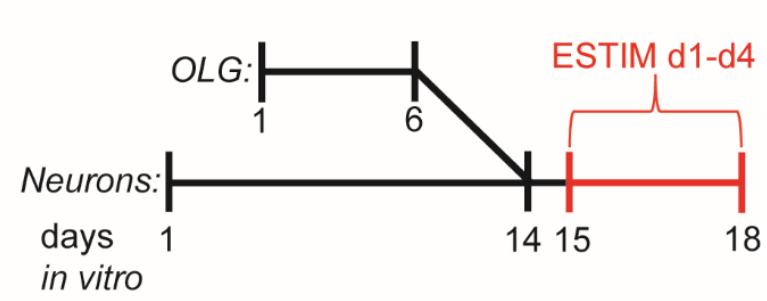

Figure 4: Time line of electrical stimulation in oligodendrocyte cultures and mixed cortical cultures

A Oligodendrocyte cultures were cultivated in culture flasks for ten days and subsequently transferred onto coverslips coated with PLL. After an additional day of growth, the cells were either treated with electrical stimulation (ESTIM) or not treated (NOSTIM) for four consecutive days. B Neuronal cultures were cultivated for 14 days on PLL coated coverslips. In parallel, oligodendrocytes were cultivated for six days in culture flasks, before being transferred onto the coverslips containing neurons. After one day of acclimatization, the mixed cortical cultures were either stimulated (ESTIM) or not stimulated (NOSTIM) for four consecutive days.

\subsubsection{Electrical stimulation in vitro}

The electrical stimulation was performed using a specialized 6-well lid (C-Dish ${ }^{\odot}$ ) from IonOptix ${ }^{\mathrm{TM}}$, which provided two graphite electrodes reaching into each well to establish an electric field within the medium. The 6-well lid was connected to an Isolated Pulse Stimulator $2100^{\odot}$ from A-M Systems ${ }^{\mathrm{TM}}$ placed on top of the $3 \mathrm{~cm}$ petri dishes containing the cells. Both, the oligodendrocyte cultures and the oligodendrocyte/neuron cocultures were stimulated in the same way with the same protocol: $10 \mathrm{~mA}, 10 \mathrm{~Hz}$ alternating current for one hour per day for four consecutive days at $37^{\circ} \mathrm{C}$ and $5 \% \mathrm{CO}_{2}$ (Fig. 4). After each stimulation, one petri dish of each experimental group was removed to perform LDH cytotoxicity tests and immunocytochemical stainings.

\subsubsection{LDH cytotoxicity test}

The CytoTox-One ${ }^{\mathrm{TM}}$ Homogenous Membrane Integrity Assay from Promega ${ }^{\mathrm{TM}}$ was used to evaluate the cell viability of the oligodendrocyte/neuron cocultures. The degree of cytotoxicity in each well could be determined by measuring the amount of lactate dehydrogenase (LDH) released into the medium by cells with damaged membranes.

In preparation for the cytotoxicity assay, the medium of each coculture petri dish was removed completely and, together with all CytotoxOne ${ }^{\mathrm{TM}}$ reagents, was brought to room temperature. As positive control (maximum LDH release) served a sample in which all cells were killed by adding a lysis buffer $(2 \mu \mathrm{l}$ per $100 \mu \mathrm{l}$ medium) for 1 hour. To start the assay, $100 \mu \mathrm{l}$ from each sample were mixed with $100 \mu \mathrm{l}$ CytoTox-One ${ }^{\mathrm{TM}}$ substrate mix in a 96-well 
plate (flat bottom) and incubated for $10 \mathrm{~min}$ at room temperature. The supernatant of each sample was vortexed before extracting $100 \mu$ to ensure an equal distribution of the LDH. The reaction was stopped by adding $50 \mu \mathrm{l}$ CytoTox-One ${ }^{\mathrm{TM}}$ stop solution to each well and the fluorescence was measured using the Safire ${ }^{\mathrm{TM}}$ plate reader from Tecan ${ }^{\mathrm{TM}}$ at $560 \mathrm{~nm}$ excitation and $590 \mathrm{~nm}$ emission. The cytotoxicity was calculated using the following formula:

$\%$ cytotoxicity $=\frac{(\text { experimental-culture medium background })}{(\text { maximum LDH release-culture medium background })}$

\subsubsection{Immunocytochemical stainings}

To prepare the cells for the immunocytochemical stainings, a fixation with $4 \%$ paraformaldehyde had to be performed. For this purpose, the cells were washed two times with PBS, fixed for 20 min in 4\% PFA and washed again two times with PBS.

Fixed cells were treated with TritonX100 (0.5\% in PBS) for 10 min to disintegrate the cell membrane and ease the access for the antibodies. After washing three times with PBS, unspecific binding sites were blocked with blocking buffer. In oligodendrocyte cultures, primary antibodies against $\mathrm{O} 4$ and MBP were used, which label oligodendrocyte lineage cells and myelin, respectively. For oligodendrocyte/neuron mixed cultures, NF200 was used in addition to $\mathrm{O} 4$ and MBP to label axonal fibers. The primary antibodies were diluted in blocking buffer and each incubated overnight at $4^{\circ} \mathrm{C}$ (table 2).

After washing three times with PBS, the fluorescence labeled secondary antibodies (table 3) were prepared in blocking solution and incubated 1 hour each. The cells were washed five times and subsequently incubated with DAPI (1:10000 in PBS) for 15 min at room temperature to stain the cell nuclei. Finally, the cells were washed three times with PBS and four times with aqua bidest before mounted on glass slides using fluorescent mounting medium.

\subsubsection{Quantification of myelin protein expression in oligodendrocyte cultures}

To quantify the number of oligodendrocytes as well as the amount of myelin expressing oligodendrocytes, the $\mathrm{O} 4$ positive and O4/MBP double positive cells were counted using ImageJ (version 1.50b, National Institute of Health, USA). The cell counts are depicted as the percentage of the total amount of DAPI positive cells.

\subsubsection{Quantification of myelinated fibers in oligodendrocyte/neuron mixed cultures}

The quantification of MBP/NF200 double positive fibers was performed using a counting grid (Fig. 5) and ImageJ (version 1.50b, National Institute of Health, USA). Only fibers crossing the counting grid were included. The amount of MBP/NF200 double positive 
fibers was compared to the amount of NF200 positive fibers to establish a myelinated/nonmyelinated fiber ratio.
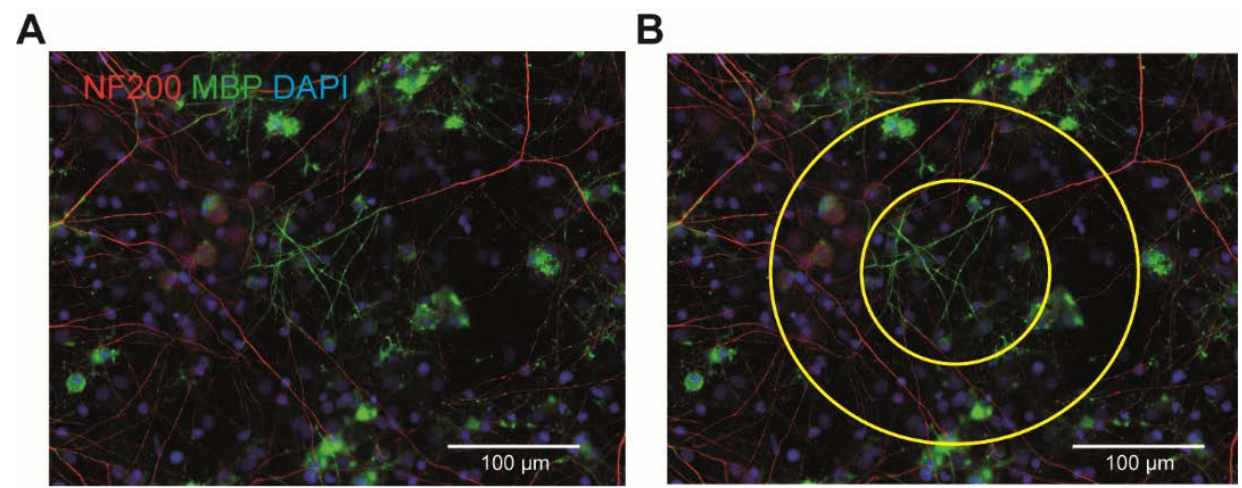

Figure 5: Quantification of myelinated axons in mixed cortical cultures

A Immunocytochemical staining for NF200 (red), MBP (green) and DAPI (blue). B Counting grid used to quantify axon number. Only axonal fibers crossing the grid at any point were counted. Scale bar: $100 \mu \mathrm{m}$

\subsection{Electrical stimulation in rodent models of demyelination}

\subsubsection{Focal demyelination model}

Focal cortical demyelinated lesions were induced by stereotactical injection of the myelin specific anti-MOG antibody Z2 in combination with mouse complement. The lesion formation and degree of remyelination was evaluated after 3, 5, 7, 10, 14 and 21 d.p.i. (days post injection). The animals were either stimulated by transcranial alternating currents or sham stimulated during the initial three days after stereotactical injection, starting at the day of injection.

\subsubsection{Animals}

A total number of 90 wildtype Lewis rats (Charles River; 195-240g) were held in groups of maximal five animals per cage with food and water ad libitum and on a 12:12 $\mathrm{h}$ light/dark cycle. In every experiment both hemispheres of each rat were treated in the same way and analyzed separately $(n=2) .24$ rats were used for an initial experiment to evaluate the lesion formation after injection of the anti-myelin antibody over the time course of three weeks without electrical stimulation (NOSTIM). Two animals were sacrificed at each time point: $3,5,7,10,14$, and 21 d.p.i. $(n=4)$. The remaining 66 animals were injected with the anti-myelin antibody and either stimulated or sham stimulated during the initial three days after stereotactical injection. Four animals of each treatment group (ESTIM, n=8 / sham, n=8) were sacrificed after 3, 5, 7, 10, 14 and 21 d.p.i. 


\subsubsection{Stereotactical injection}

Animals were anesthetized i.p. by injecting a ketamine /xylazine solution (ketamine: $60 \mathrm{mg} / \mathrm{kg}$ body weight; xylazine: $8 \mathrm{mg} / \mathrm{kg}$ body weight) and mounted in a stereotactical frame. The shaved head was firmly fixed and the skin above the skull was cut open with a scalpel. The skin was held in place by clamps and a small hole for the injection was drilled into the skull $1 \mathrm{~mm}$ caudal and $2 \mathrm{~mm}$ lateral of the bregma on each side of the head. After exposing the brain, a glass capillary with a diameter of $0.05-0.1 \mathrm{~mm}$ was introduced $1.5 \mathrm{~mm}$ deep into the brain, filled with $1 \mu$ of the anti-MOG antibody solution. The solution was injected very slowly and carefully at a steady pace. The glass capillary was left in the brain for one minute before withdrawing to prevent any backflow of the liquid. Monastral Blue was used to mark the injection site. Afterwards, the skin was sutured and the rat was placed in a cage and injected with an analgetic (Temgesic ${ }^{\odot}, 1 \mu$ per $1 \mathrm{~g}$ body weight).

\subsubsection{Electrical Stimulation}

The rats were stimulated for 10 min each day with $100 \mu \mathrm{A}$ and $10 \mathrm{~Hz}$ alternating current in a transcranial fashion for three consecutive days, starting with the day of the stereotactical injection. 30 min prior to the stimulation the rats were injected with an analgetic (Temgesik). In preparation to the stimulation the rat was placed on the stomach on a heating plate to prevent hypothermia and was anesthetized with Isoflurane by using an Isofluranepump (Sigma Delta Vaporizer ${ }^{\circledR}$ ). To reduce the impedance for the stimulation, the fissure of the previous stereotactical injection was reopened and the stimulating electrode $\left(1 \mathrm{~cm}^{2}\right)$, to which electrode conducting gel was added, was placed on top of the intact skull. The reference electrode $\left(35 \mathrm{~cm}^{2}\right)$ was placed under the thorax. After the stimulation, the electrodes were removed, the wound cleaned of any remaining electrode gel and sutured. This procedure was repeated on the next two days to achieve a total of three days of stimulation. The procedure of the sham stimulation is identical, with the exception that the stimulator was not turned on during the stimulation.

\subsubsection{Chronic cuprizone model}

A chronic cuprizone model was used to evaluate the effect of electrical stimulation on delayed remyelination. The animals were fed with cuprizone for an extended period of 13 weeks to ensure a delayed remyelination. Subsequently, they were put on normal chow to induce remyelination and either stimulated or sham stimulated for five days or ten days (Fig. $6)$. 


\subsubsection{Animals}

A total of 35 adult wildtype C57BL/6J mice (Charles River) aged between six and eight weeks (17 $\pm 1.5 \mathrm{~g}$ ) were held in groups of maximal eight animals per cage with food and water ad libitum and on a 12:12 h light/dark cycle. All mice were fed cuprizone for 13 weeks. Five mice were removed from the experiment due to severe weight loss $(>20 \%$ of starting weight). The remaining 30 animals were put on normal chow after 13 weeks of cuprizone diet for either five or ten days and separated into 4 groups: $5 d$ ESTIM, $n=8 ; 5 d$ sham, n=8; 10d ESTIM, n=7; 10d sham, n=7 (Fig. 6).

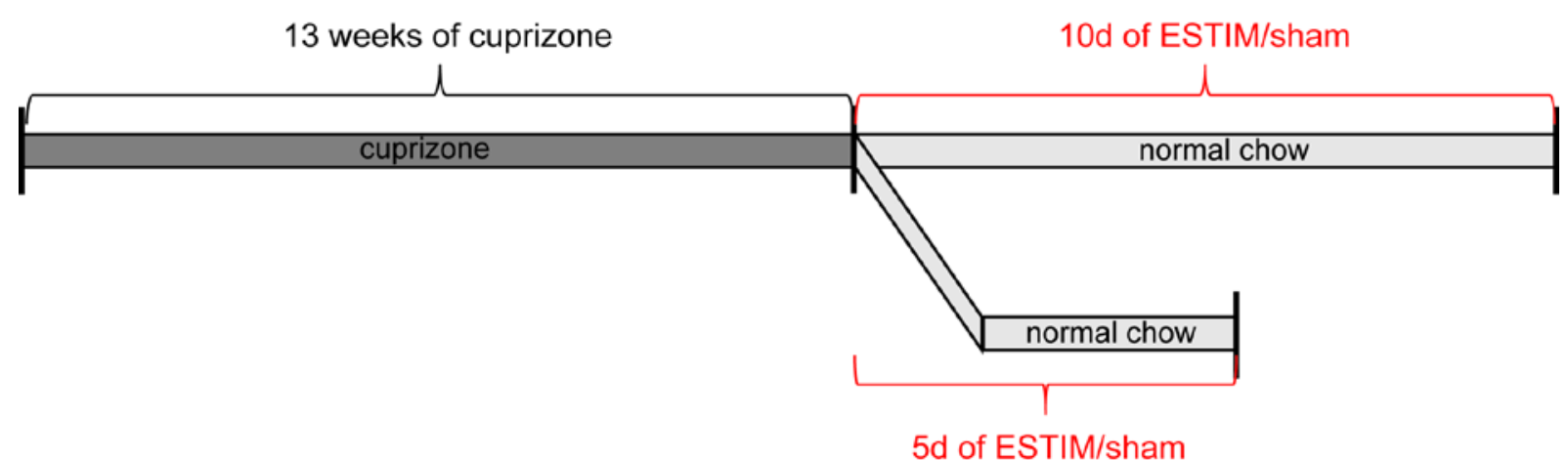

Figure 6: Time line of the chronic cuprizone model

Mice were fed $0.25 \%$ cuprizone for 13 weeks, followed by either five or ten days on a cuprizone-free diet with ESTIM or sham treatment.

\subsubsection{Electrical stimulation}

The mice were stimulated for 10 min each day with $100 \mu \mathrm{A}$ and $10 \mathrm{~Hz}$ alternating current in a transcranial fashion for five or ten days, starting one day after cuprizone removal. In preparation to the stimulation the mouse was placed on the stomach on a heating plate to prevent hypothermia and was anesthetized with isoflurane by using an isoflurane-pump. The stimulating electrode $\left(1 \mathrm{~cm}^{2}\right)$, to which electrode conducting gel was added, was placed on top of the shaved head above the bregma. The reference electrode $\left(35 \mathrm{~cm}^{2}\right)$ was placed onto the skin under the thorax. After the stimulation, the electrodes were removed and the head cleaned of any remaining electrode gel. The procedure of the sham stimulation was identical, only the stimulator was not turned on during the stimulation.

\subsubsection{Histopathology}

\subsubsection{Histochemical stainings}

If not indicated otherwise, the following steps were performed at room temperature. Animals were anaesthetized by injecting an overdose of $14 \%$ chloral hydrate and transcardially perfused with $0.1 \mathrm{M}$ PBS followed by $4 \%$ paraformaldehyde. Brains were removed and post fixed in $4 \%$ paraformaldehyde for two days at $4{ }^{\circ} \mathrm{C}$. Afterwards, brains 
were dissected and cut into 3-5 mm thick slices, which were dehydrated in an ascending ethanol series before being embedded in paraffin. Brain slices were cut with $0.5 \mu \mathrm{m}$ width and stained with Hematoxylin / eosin (HE) for a general overview of the tissue, Luxol fast blue / periodic acid Schiff (LFB/PAS) for detection of myelin and Bielschowsky for detection of nerve fibers, as described in the following section. In preparation for histochemical stainings, the brain slices were placed on microscope slides, deparaffinized (three times 3 min in Xylol and Isoxylol each) and rehydrated in a descending ethanol series (100\% ethanol, 90\%, 70\%, 50\%, aqua bidest., 3 min each).

\section{Hematoxylin / eosin staining (HE)}

The first reagent of the HE staining, hematoxylin, is a basic dye, which stains basophilic structures like the DNA or the rough endoplasmic reticulum. The second component, eosin, is an alcohol based acid and colors eosinophilic structures like proteins. Following the descending ethanol series the slices were processed in Mayer's hemalum for 5 min, washed in aqua dest., differentiated in $1 \% \mathrm{HCl}$ and blued with tap water for $10 \mathrm{~min}$. Subsequently, the slices were treated with $1 \%$ eosin for 5 min, washed with aqua dest., dehydrated through an ascending ethanol series and mounted.

\section{Luxol fast blue / periodic acid Schiff (LFB/PAS) staining}

A detailed description of the LFB/PAS staining can be found under 2.2.5.1.

\section{Bielschowsky's silver staining}

The Bielschowsky staining colors nerve fibers and was used to detect possible axonal loss due to necrosis after stereotactical injections. Following the deparaffination, the slices were treated with a $20 \%$ silver nitrate solution for 20 min followed by $15 \mathrm{~min}$ in a $20 \%$ silver nitrate solution to which $32 \%$ ammonia was added drop wise until the resulting precipitations were cleared. Subsequently, the slices were transferred to into aqua bidest., to which initially three drops of $32 \%$ ammonia were added, followed by ten drops of the development solution, which started the development of the staining. The reaction was stopped with aqua dest., the slices were incubated in $2 \%$ sodium thiosulfate for $2 \mathrm{~min}$, washed with tap water and dehydrated in an ascending ethanol series before mounted with glass coverslips.

\subsubsection{Immunohistochemical stainings}

The immunohistochemical stainings were performed as explained under 2.2.5.2. The primary antibodies were used in the combinations MBP, Olig2/BrdU/MBP and Olig2/p25/MBP for the focal demyelination model and Olig2/NogoA/BrdU for the chronic 
cuprizone model. A complete list of all primary and secondary antibodies as well as the pretreatments can be found in table 2 and table 3 , respectively.

\subsubsection{Data analysis}

To evaluate the different cell populations of the histochemical and immunohistochemical stainings, whole slide pictures were made by using the Olympus VS120 fluorescence slide scanner with a 200 -fold magnification. To reduce the file size, the pictures were cropped and converted into tiff files. The quantification of the oligodendrocyte cell populations of the chronic cuprizone model as well as oligodendrocyte populations and the demyelinated lesion formation of the focal demyelination model was performed using ImageJ (version 1.50b). Four separate counting areas were defined to evaluate the oligodendrocyte populations, depending on their position in relation to the demyelinated lesion (Fig. 7): demyelinated lesion area (lesion), perilesional area (peri-lesion, only at 3 and 5 d.p.i, defined as the area surrounding the lesion by up to $200 \mu \mathrm{m}$ ), remyelinated area (RM area, only at 7 - 21 d.p.i) and normal appearing gray matter (NAGM). The statistical analysis was performed using GraphPad Prism for Windows version 5.0.0. t-Tests were used to compare two experimental groups with each other. In case of non-Gaussian distribution, a Mann-Whitney test was performed. For comparing more than two experimental groups, a Two-way ANOVA followed by Sidak's multiple comparisons test was performed. To analyze the lesion formation over time for each experimental group separately, a Kruskal-Wallis test followed by Dunn's multiple comparisons test was performed. A $p$ value of $\leq 0.05$ was considered significant and a $p$ value between $0.1-0.051$ was considered a trend. Data are presented as mean \pm SEM. 
d3

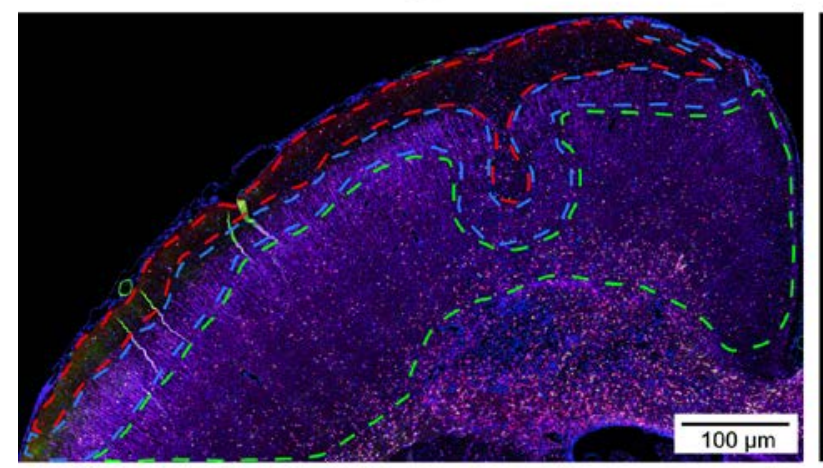

---- DM lesion

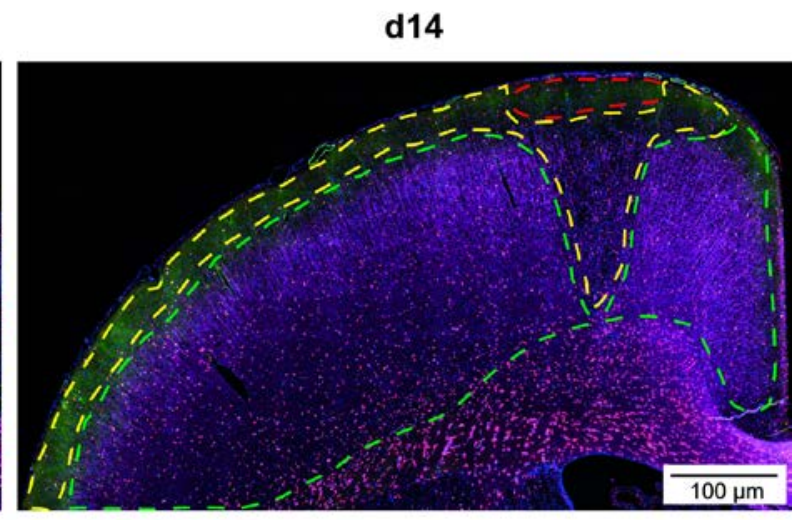

RM area

NAGM

Figure 7: Schematic overview of the four separate counting areas used to evaluate OLG populations in the focal EAE model

To quantify the OLG populations in relation to their distance towards the demyelinated lesion, four separate areas were defined: demyelinated lesion area (lesion), perilesional area (peri-lesion, 3 and 5 d.p.i.), remyelinated area (RM area, 7 - 21 d.p.i.) and normal appearing gray matter (NAGM). 


\section{Part III - Methods}

Transcranial electrical stimulation in healthy human subjects 


\subsection{Transcranial electrical stimulation in healthy human subjects}

Transcranial alternating current stimulation (tACS) was applied in an electrical theta burst (eTBS) fashion to the motor cortex of healthy human subjects to evaluate the impact of electrical stimulation on corticospinal excitability.

\subsubsection{Subjects}

For the first part of the study which used $5 \mathrm{~mA}$ tACS, 17 healthy subjects aged $24.4 \pm$ 3.4 years (9 females / 8 males) were recruited, of which three were left-handed. Due to painful skin sensations during the electrical stimulation, two subjects from the first group (1 female / 1 male) withdraw from the clinical study. 14 healthy subjects aged $24.9 \pm 4.5$ years (8 females / 6 males; all right handed) were recruited for the second part of the study. This second study part utilized higher current intensity of $10 \mathrm{~mA}$, therefore a sensation questionnaire was introduced, which each participant had to complete after every session. Of the second group of participants, four ( 3 female / 1 male) withdraw from the experiment due to painful skin sensations. None of the volunteers matched any exclusion criteria like a history of neurological disease, pregnancy, metallic implants or took any medications at the time of the study. They all gave written informed consent and were compensated for participating. The investigation was approved by the Ethics Committee of the Medical Faculty of University of Göttingen, and conformed to the principals laid down in the Declaration of Helsinki.

\subsubsection{Transcranial magnetic stimulation}

To measure potential changes in corticospinal excitability before and after eTBS, monophasic single-pulse transcranial magnetic stimulation (TMS) was used as described by Batsikadze et al., 2013 and Sommer et al., 2013. A figure-of-eight magnet coil (diameter of one winding: $70 \mathrm{~mm}$; peak magnetic field: $2.2 \mathrm{~T}$ ) was held tangentially to the scalp over the hand region of the motor cortex, with the handle pointing backwards and laterally at $45^{\circ}$ from midline to induce muscle evoked potentials (MEP). Two $\mathrm{Ag}-\mathrm{AgCl}$ surface electrodes were applied to the contralateral first dorsal interosseous (FDI) muscle in a belly-tendon montage and used to record MEP induced by single pulse TMS. To determine the optimal position for the coil (hotspot), single pulse TMS was induced until $1 \mathrm{mV}$ MEP were recorded at the FDI. A dermatological tested tattoo marker was used to mark the hotspot on the participants' scalp, therefore providing a fix point by which the coil can be repositioned at each measurement. Resting motor threshold (RMT) was defined as the minimum stimulator output needed to elicit a MEP response of $\pm 0.5 \mathrm{mV}$ in the relaxed FDI muscle in at least 5 out of 10 consecutive trials. The signals were amplified and band-passed filtered $(2 \mathrm{~Hz}$ to $2 \mathrm{kHz}$, 
sampling rate $5 \mathrm{kHz}$, amplifier gain: 1000), digitized by a power 1401 AD converter, controlled by Signal Software and stored for offline analysis.

\subsubsection{Experimental design}

The study was performed in a double-blinded, cross-over design with each eTBS protocol applied in a randomized order. The participants were seated in a comfortable chair with head and arm rests and were instructed to relax whenever necessary. Prior to the eTBS treatment, two separate TMS measurements were performed with a ten-minute break in between. The mean of the resulting MEP responses were calculated to establish a reliable baseline. Further TMS measurements were performed immediately after the stimulation and every $10 \mathrm{~min}$ for up to $60 \mathrm{~min}$ to evaluate eTBS after-effects. The experiments were performed either between 09:00-12:00 a.m. or 03:00-07:00 p.m. and a minimum of one week time was set between each session with the same subject to prevent any cross-session effects.

\subsubsection{Electrical theta burst stimulation}

To apply eTBS, one electrode ( $3 \times 4 \mathrm{~cm}$; cable facing posterior direction) was placed over the left primary motor cortex (M1 region), while the other electrode (6 $88 \mathrm{~cm}$; cable facing lateral direction) was placed over the contralateral orbit. Both electrodes were prepared with $T e n 20^{\circledR}$ conductive electrode paste to reduce the impedance during the stimulation. The eTBS protocols were encoded by Signal Software version 2.16 and applied by a DS5 isolated constant current stimulator. In the first part of the study, three different eTBS protocols were used with an intensity of $5 \mathrm{~mA}$. The eTBS protocols were adapted from the original study performed by Huang et al. in 2005: intermittent eTBS (eiTBS), intermediate eTBS (eimTBS) and continuous eTBS (ecTBS; Fig. 8). Each eTBS pattern consisted of three stimulation pulses at $50 \mathrm{~Hz}$, repeated every $200 \mathrm{~ms}$ for a total of 600 pulses each. Each of these three pulses consisted of five sinusoidal bursts at $5 \mathrm{kHz}$, repeated every millisecond. eiTBS uses a two-second train of eTBS repeated every $10 \mathrm{~s}$ for a total of $190 \mathrm{~s}$. eimTBS uses a five-second train of eTBS, repeated every $15 \mathrm{~s}$ for a total of $110 \mathrm{~s}$. The ecTBS pattern consists of a 40-second train of uninterrupted eTBS. As a forth protocol, sham stimulation was introduced, in which the stimulator was programmed not to induce any currents. All stimulation protocols, including sham, were set to last 240 seconds to ensure an optimal blinding.

The second part of the study consisted of three altered ecTBS protocols: ecTBS with sinusoidal bursts of $1 \mathrm{~ms}$ duration and $10 \mathrm{~mA}$ intensity, ecTBS with sinusoidal bursts of $5 \mathrm{~ms}$ duration and $5 \mathrm{~mA}$ intensity and ecTBS with sinusoidal bursts of $5 \mathrm{~ms}$ duration and $10 \mathrm{~mA}$ intensity. 


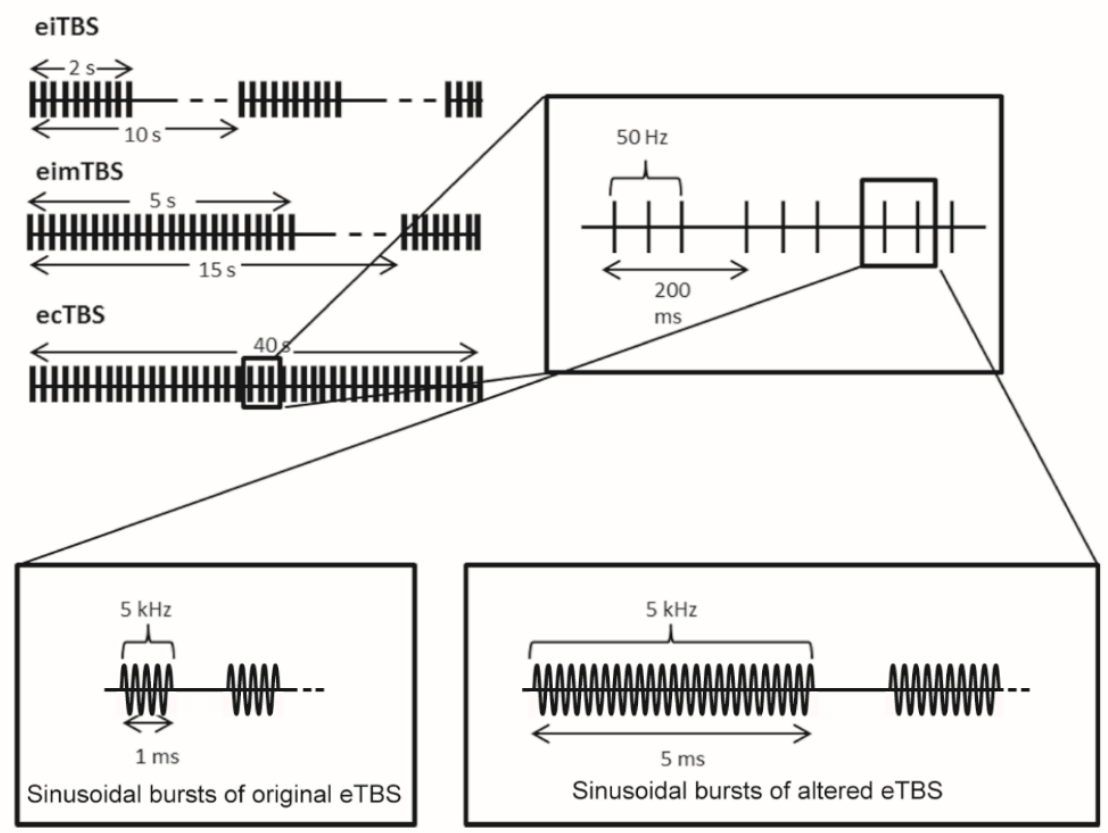

Figure 8: Schematic illustration of the eTBS protocols

Each eTBS pattern consists of three pulses given at $50 \mathrm{~Hz}$, repeated every $200 \mathrm{~ms}$. Intermittent eTBS (eiTBS) uses a $2 \mathrm{~s}$ train of eTBS, repeated every $10 \mathrm{~s}$. Intermediate eTBS (eimTBS) uses a $5 \mathrm{~s}$ train of eTBS, repeated every $15 \mathrm{~s}$. Continuous eTBS (ecTBS) uses a $40 \mathrm{~s}$ train of uninterrupted eTBS. The first part of the study uses sinusoidal bursts with a length of $1 \mathrm{~ms}$ at $5 \mathrm{kHz}$, whereas in the second part of the study a burst length of $5 \mathrm{~ms}$ at $5 \mathrm{kHz}$ was used in addition to the original sinusoidal bursts. The basic TBS pattern were obtained and modified from Huang et al. (2005). This figure is part of a clinical study, published in Frontiers in Human Neuroscience (Kunz et al. 2017).

\subsubsection{Data analysis}

Data were analyzed using GraphPad Prism for Windows version 5.0.0. Repeated measures ANOVA with the parameters TYPE (type of stimulation) and TIME (time course of $60 \mathrm{~min}$ ) was used on MEP data normalized to the respective baseline to compare each eTBS condition vs. sham. Kruskal-Wallis test followed by Dunn's multiple comparison test was performed to compare specific time points to baseline. A $p$ value of $\leq 0.05$ was considered significant and a $p$ value of $0.1-0.051$ was considered a trend. Data are presented as mean \pm SEM. 


\section{Part I-Results}

Influence of autoimmune inflammation on remyelination in Cuprizone-induced demyelination 


\subsection{Influence of autoimmune inflammation on remyelination in Cuprizone- induced demyelination}

To evaluate the remyelination processes after Cuprizone-induced demyelination (two weeks of $0.25 \%$ Cuprizone, followed by three weeks of $0.15 \%$ ) with and without the activation of the adaptive immune system, 77 mice were separated into four groups: Cuprizone treated animals (Cup, $n=29$ ), Cuprizone treated animals immunized with MOG $_{35-55}$ peptide (Cup-EAE, $n=32$ ), Cuprizone treated animals immunized with ovalbumin instead of MOG $_{35-55}$ peptide (Cup-Ova, $n=12$ ) and naïve control animals $(n=4)$. The Cup-Ova mice served as controls to Cuprizone/MOG treated animals during the initial set of experiments in which the extent of the activation of the immune system was evaluated. Naïve controls were used for electron microscopic analysis of the corpus callosum (CC) to compare the density of myelinated fibers to Cup and Cup-EAE mice.

\subsubsection{Cuprizone administration for 4.5 weeks leads to demyelination in the corpus callosum in Cup and Cup-EAE animals}

The copper chelator Cuprizone was administered to all animals except the naïve control mice for 4.5 weeks. To evaluate the extent of demyelination (DM) in the CC, LFBPAS stainings of the mouse brains of Cup and Cup-EAE animals were performed (Fig. 9 AC). For a more precise evaluation, the CC was separated into a medial and two lateral parts. Semi-quantitative analysis using a well-established demyelination score (DM score, table 11; Hiremath et al., 1998) revealed a severe demyelination in the medial as well as the lateral CC of both experimental groups (Cup medial: $2.71 \pm 0.29$, Cup lateral: $2.86 \pm 0.14$; Cup-EAE medial: $2.75 \pm 0.16$, Cup-EAE lateral: $3 \pm 0$; Fig. $9 \mathrm{D}-\mathrm{F}$ ) to the same extent. 

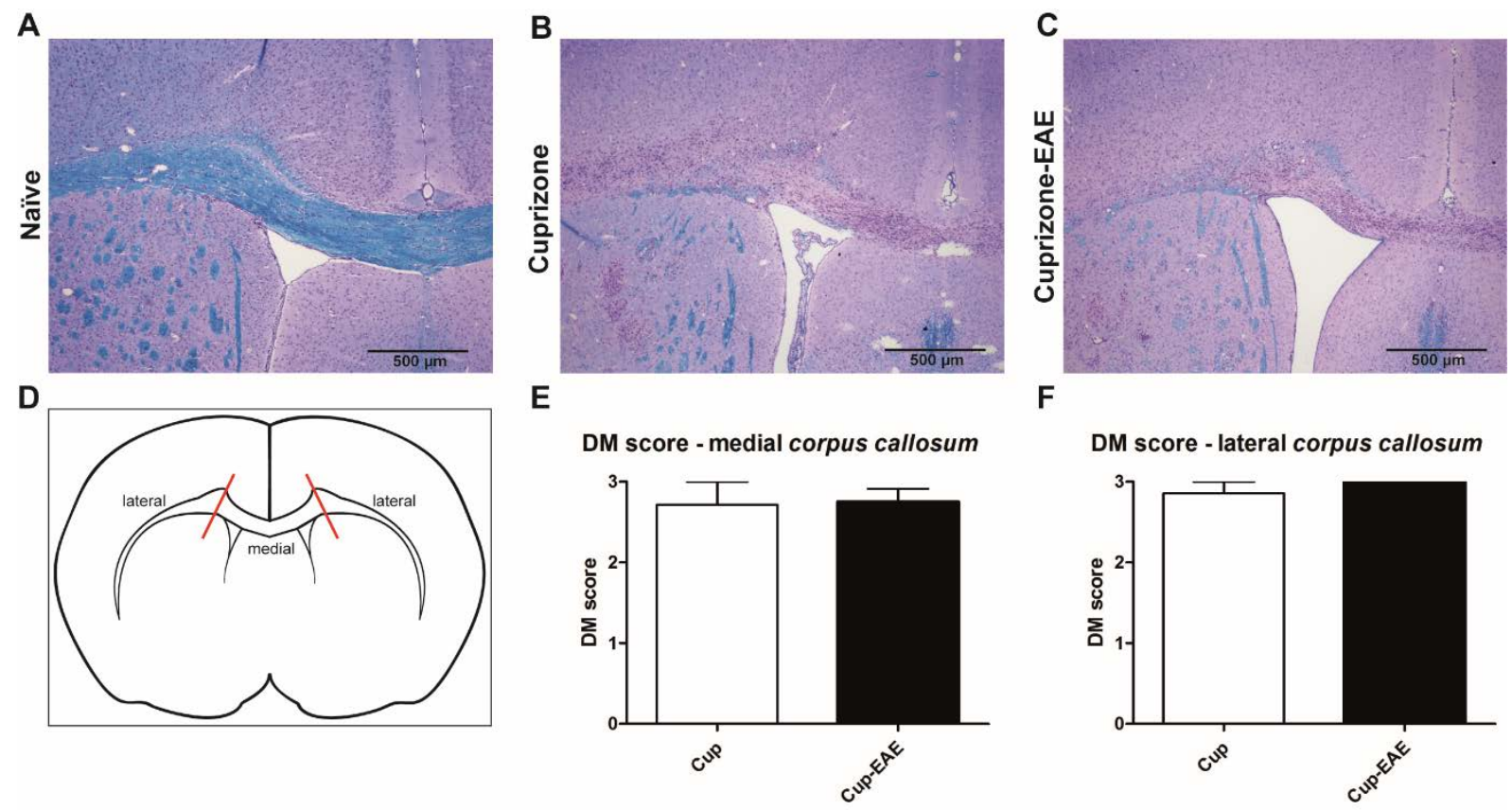

F

DM score - lateral corpus callosum

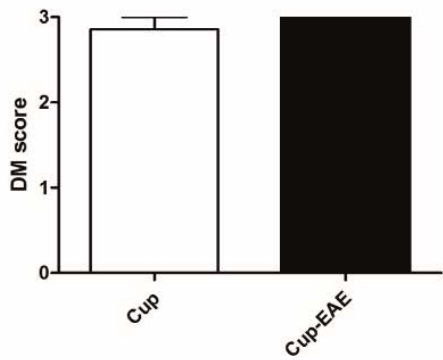

Figure 9: 4.5 weeks of Cuprizone administration causes severe demyelination of the $\mathrm{CC}$ in Cup and Cup-EAE mice

LFB-PAS stainings of the CC of A naïve ( $n=4)$, B Cup ( $n=7)$ and C Cup-EAE mice $(n=7)$. Cup and Cup-EAE animals were fed Cuprizone for 4.5 weeks, while naïve animals were fed normal chow. $\mathbf{D}$ Schematic illustration of the mouse brain with the medial and lateral parts of the CC separated by red lines. Semi-quantitative analysis demonstrated an extensive demyelination in $\mathbf{E}$ the medial and $\mathbf{F}$ the lateral CC of Cup as well as Cup-EAE animals. No significant difference was found by comparing the two treatment groups using the Mann-Whitney test. Data are shown as means \pm SEM.

\subsubsection{Increased infiltration and activation of immune competent cells in Cuprizone/MOG treated animals}

In the first set of experiments, the immune response after 4.5 weeks of Cuprizone administration with and without $\mathrm{MOG}_{35-55}$ immunization was quantified by flow cytometric analysis (Fig. 10). The total amount of T cells in the CNS of Cup-EAE mice was significantly increased (34316 \pm 7917 T cells, $\left.{ }^{* *} p<0.001\right)$ compared to the low number of $T$ cells found in Cuprizone treated mice $(697.6 \pm 132.4 \mathrm{~T}$ cells, Fig. $10 \mathrm{~A})$. Cup-Ova mice demonstrated a slightly, but not significantly, elevated level of T cells in the CNS compared to Cup animals (7494 \pm 2570 T cells, $p=0.209$ ), while compared to Cup-EAE animals the $T$ cell population was still slightly, but not significantly, smaller ( $p=0.125$, Fig. 10 A). Following $T$ cell activation and infiltration after immunization with $\mathrm{MOG}_{35-55}$ peptide, an increase of $\mathrm{NK}$ cells was expected as an immediate immune response. Indeed, the total cell count of NK cells was significantly increased in Cup-EAE mice (2947 \pm 538.5 NK cells) compared to Cup (164.3 \pm 29.05 NK cells, $\left.{ }^{* \star \star} p<0.001\right)$ and Cup-Ova $\left(710.8 \pm 200.3\right.$ NK cells, ${ }^{* \star} p<0.01$, Fig. 10 B) animals. No increase in NK cells was registered following immunization with Ovalbumin 
alone. Further important indicators for an activated immune system are B cells. Similarly to $T$ - and NK cells, the total number of $B$ cells was found significantly increased in Cup-EAE mice (6150 \pm 1017 B cells) compared to Cup $\left(126.4 \pm 22.29\right.$ B cells, $\left.{ }^{* \star *} p<0.001\right)$ and CupOva animals ( $2135 \pm 1048$ B cells, ${ }^{*} p<0.05$, Fig. $10 \mathrm{C}$ ). The B cell numbers found in Cup-Ova mice did not differ significantly to animals in the Cup group. The total amount of granulocytes, an important compartment of the innate immune system, registered as well a significant increase in the Cup-EAE group (36599 \pm 8223 granulocytes) compared to Cup mice (519.8 \pm 177 granulocytes, ${ }^{\star \star \star} p<0.001$, Fig. $\left.10 \mathrm{D}\right)$. The granulocyte number in Cup-EAE animals was slightly increased compared to Cup-Ova animals (9877 \pm 2696 granulocytes, $p=0.224$, Fig. $10 \mathrm{D}$ ). Further FACS stainings indicated an increase in the total number of inflammatory monocytes after immunization with MOG $_{35-55}$ peptide $(21703 \pm 4860$ infl. monocytes) compared to Cup (203.3 \pm 42.64 infl. monocytes, $\left.{ }^{* *} p<0.01\right)$ and Cup-Ova (5994 \pm 2050 infl. monocytes, ${ }^{*} p<0.05$, Fig. 10 E) mice. The amount of inflammatory monocytes in Cup-Ova animals did not differ significantly from the cell count of Cuprizone only treated animals. The total number of microglia, however, did not differ significantly in any of the three treatment groups (Cup: $1206 \pm 204.3$ microglia, Cup-Ova: $1343 \pm 323.2$ microglia, Cup-EAE: $854.7 \pm 140$ microglia, $p>0.6$, Fig. 10 F). 

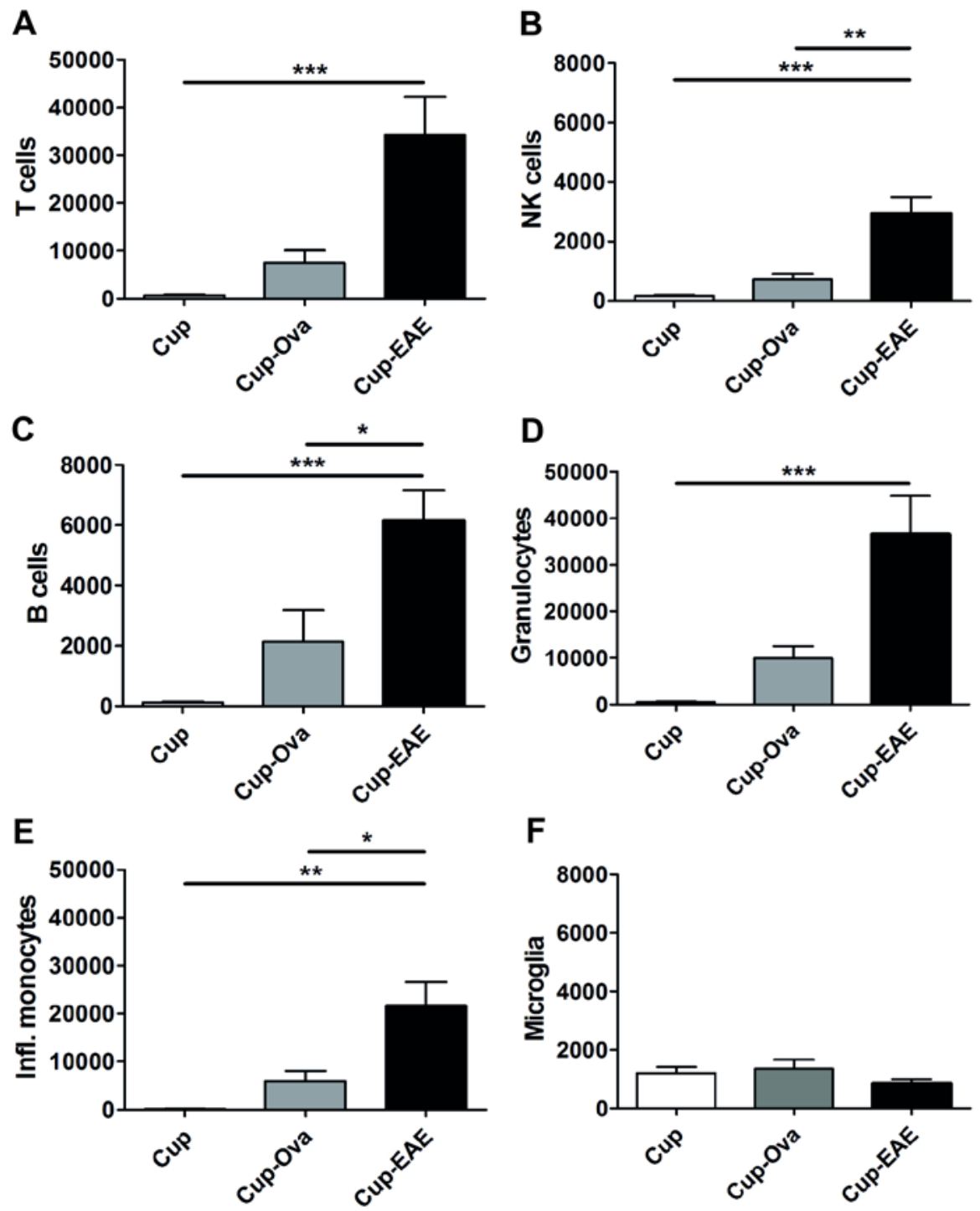

Figure 10: Immunization with $\mathrm{MOG}_{35-55}$ peptide leads to increased infiltration of immune
competent cells Flow cytometric analysis of inflammatory cells in Cup $(n=6)$, Cup-Ova $(n=6)$ and Cup-EAE $(n=9)$ mice. Following immunization with $\mathrm{MOG}_{35-55}$ peptide, a significant increase in the total number of $\mathbf{A} T$ cells (CD45 $\left.{ }^{+} \mathrm{CD}^{+} ;{ }^{* \star *} p<0.001\right)$, B NK cells $\left(\mathrm{CD} 45^{+} \mathrm{Nk} 1.1^{+} \mathrm{Nkp} 46^{+} ;{ }^{* \star *} p<0.001\right)$, C B cells $\left(\mathrm{CD} 45^{+} \mathrm{CD} 19^{+}\right.$ CD3; $\left.{ }^{* *} p<0.001\right)$, D granulocytes $\left(C D 45^{+} \mathrm{CD} 11 \mathrm{~b}^{+} \mathrm{Ly} 6 \mathrm{G}^{+} ;{ }^{* \star *} p<0.001\right)$ and $\mathbf{E}$ inflammatory monocytes

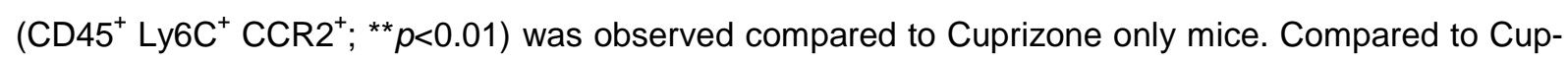
Ova mice, Cup-EAE animals demonstrated a significant increase in $T$ cells $\left({ }^{*} p<0.05\right)$, NK cells $\left({ }^{*} p<0.01\right)$, B cells $\left({ }^{*} p<0.05\right)$ and inflammatory monocytes $\left({ }^{*} p<0.05\right)$. F The total number of microglia

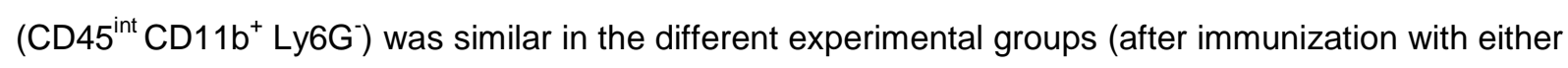
ovalbumin or $\mathrm{MOG}_{35-55}$ peptide). Data were analyzed by One-way ANOVA followed by Bonferroni's multiple comparison test. For non-parametric data, the Kruskal-Wallis test followed by Dunn's multiple comparison test was performed. Data are shown as mean \pm SEM. 


\subsubsection{The number of OPC and mature OLG is largely unaffected by EAE induction}

As demonstrated earlier, the number of T cells, which are known to contribute to an inflammatory milieu by releasing pro-inflammatory cytokines like IFN- $\gamma$, was increased in Cup-EAE animals. To assess whether the inflammatory environment influences the OLG survival in the demyelinated corpus callosum, immunohistochemistry for the myelinassociated neurite growth inhibitor NogoA and the transcription factor Olig2 were performed (Fig. 11 A). OLG lacking the NogoA marker were considered OPC, while OLG expressing NogoA were considered mature OLG. Animals were sacrificed after either 4.5 weeks of Cuprizone treatment at the demyelination time point (DM) or after five additional days on normal chow at the remyelination time point (RM) to evaluate OLG populations.

The average number of OPC found in the medial CC of Cup mice at the DM time point remained similar compared to Cup-EAE mice (Cup DM: $705.1 \pm 88.97$ Olig2 $^{+}$NogoA $^{-}$ cells $/ \mathrm{mm}^{2}$, Cup-EAE DM: $618.0 \pm 74.86$ Olig2 $^{+} \mathrm{NogoA}^{-}$cells $/ \mathrm{mm}^{2}$, Fig. 11 B) and decreased after 5 days of RM for both groups (Cup RM: $315.0 \pm 30.23$ Olig2 ${ }^{+} \mathrm{NogoA}^{-}$cells $/ \mathrm{mm}^{2}$, ${ }^{* \star *} p<0.001$; Cup-EAE RM: $405.2 \pm 36.06$ Olig2 ${ }^{+} \operatorname{NogoA}^{-}$cells $/ \mathrm{mm}^{2},{ }^{*} p<0.05$; Fig. 11 B). Similarly, OPC counts in the lateral area of the $\mathrm{CC}$ of Cup-EAE animals remained comparable to Cup only mice at both time points (Cup DM: $441.5 \pm 58.91$ Olig2 $^{+} \mathrm{NogoA}^{-}$ cells $/ \mathrm{mm}^{2}$, Cup-EAE DM: $356.6 \pm 34.33$ Olig2 $^{+} \mathrm{NogoA}^{-}$cells $/ \mathrm{mm}^{2}$, Cup RM: $203 \pm 17.37$ Olig2 ${ }^{+} \mathrm{NogoA}^{-}$cells $/ \mathrm{mm}^{2}$, Cup-EAE RM: $318.3 \pm 47.48$ Olig2 ${ }^{+} \mathrm{NogoA}^{-}$cells $/ \mathrm{mm}^{2}$, Fig. $11 \mathrm{C}$ ). However, only the Cup group demonstrated a significant drop in the OPC number after five days of RM ( $\left.{ }^{* *} p=0.01\right)$, while the OPC number of Cup-EAE animals remained stable.

Quantifying the density of mature OLG in the medial CC, a different situation was observed. The overall number of mature OLG was in general lower than the OPC number, and a Two-way ANOVA revealed a difference in the density of $\operatorname{NogoA}^{+}$OLG between Cup and Cup-EAE animals (Cup DM: $265.3 \pm 83.7$ NogoA $^{+}$cells $/ \mathrm{mm}^{2}$, Cup-EAE DM: $169.5 \pm$ $41.97 \mathrm{NogoA}^{+}$cells $/ \mathrm{mm}^{2}$, Cup RM: $241.7 \pm 24.98 \mathrm{NogoA}^{+}$cells $/ \mathrm{mm}^{2}$, Cup-EAE RM: $88.98 \pm$ $23.49 \mathrm{NogoA}^{+}$cells $/ \mathrm{mm}^{2},{ }^{*} p<0.05, F(1,23)=7.158$, Fig. $\left.11 \mathrm{D}\right)$. However, a post-test with Sidak's multiple comparisons test demonstrated no significant effects between Cup and CupEAE animals but a trend toward a decrease in the number of $\operatorname{Nogo}^{+}$OLG after five additional days of RM compared to the Cup group ( $p=0.0521$; Fig. $11 \mathrm{D}$ ). No difference between Cup and Cup-EAE animals was observed in the lateral CC at any time point, and the number of $\mathrm{NogoA}^{+}$OLG did not change significantly over time (Cup DM: $118.5 \pm 33.4$ Nogo $^{+}$cells $/ \mathrm{mm}^{2}$, Cup-EAE DM: $54.65 \pm 12.74 \mathrm{NogoA}^{+}$cells $/ \mathrm{mm}^{2}$, Cup RM: $121.2 \pm 14.93$ NogoA ${ }^{+}$cells $/ \mathrm{mm}^{2}$, Cup-EAE RM: $99.03 \pm 25.51 \mathrm{NogoA}^{+}$cells $/ \mathrm{mm}^{2}$, Fig. $11 \mathrm{E}$ ). 
A

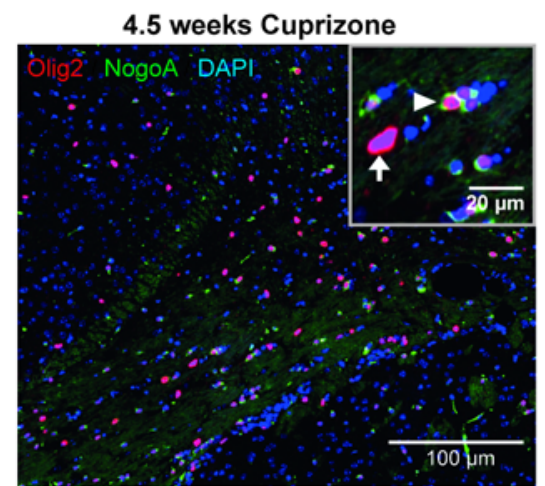

B

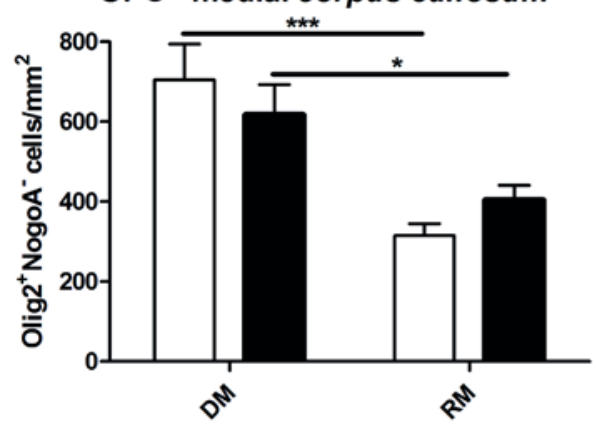

4.5 weeks Cuprizone-EAE

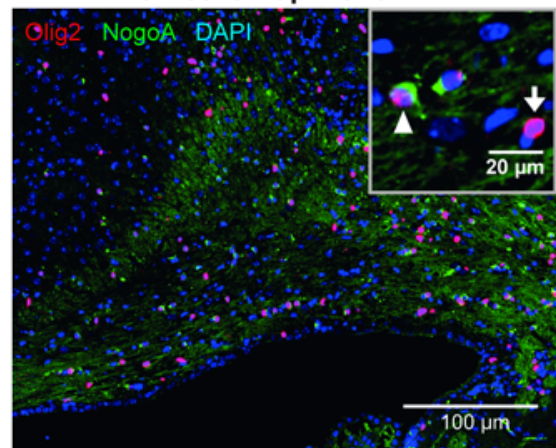

C

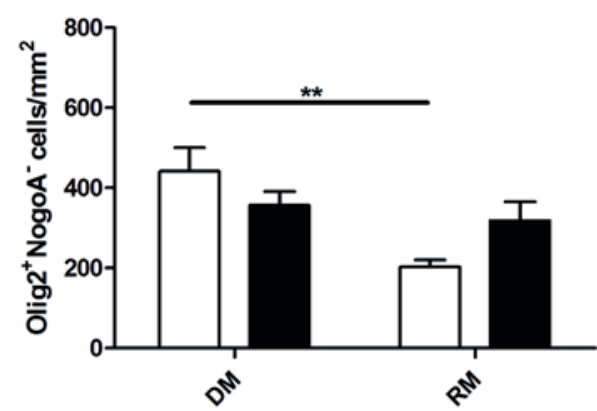

D Mature OLG - medial corpus callosum E Mature OLG - lateral corpus callosum
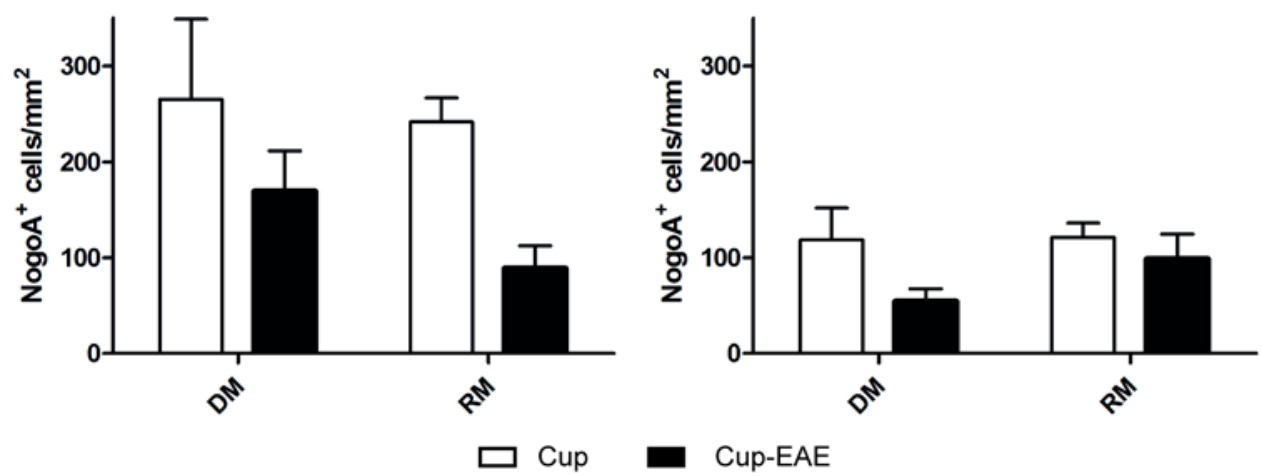

Figure 11: Less mature OLG in the medial corpus callosum at the RM time point after EAE induction

Evaluation of OPC and mature OLG numbers by immunohistochemical stainings against Olig2 and NogoA with (Cup-EAE DM $n=6$, Cup-EAE RM $n=7$ ) and without $\mathrm{MOG}_{35-55}$ immunization (Cup DM $n=7$, Cup RM $n=7$ ). A Immunohistochemical staining of the CC of Cup and Cup-EAE mice after 4.5 weeks of Cuprizone administration with antibodies against Olig2 (arrow) and NogoA (arrow head; scale bar: $100 \mu \mathrm{m}$, inset scale bar: $20 \mu \mathrm{m}$ ). B No significant effect on OPC numbers in the medial CC after EAE induction compared to Cup. Both treatment groups depicted a significantly lower number of OPC at the RM time point (Cup: ${ }^{* \star *} p<0.001$, Cup-EAE: ${ }^{*} p<0.05$; Two-way ANOVA followed by Sidak's multiple comparisons test). C No significant effect on OPC numbers in the lateral area of the CC after immunization with $\mathrm{MOG}_{35-55}$ peptide compared to Cup. OPC of the Cup group are significantly reduced at the RM time point ${ }^{* \star} p<0.01$; Two-way ANOVA followed by Sidak's multiple comparisons test). D The Two-way ANOVA revealed a significant effect in the number of mature OLG in the medial CC between Cup and Cup-EAE mice ( ${ }^{*} p<0.05, F(1,23)=7.158$; Two-way ANOVA). After five days of RM, Cup-EAE animals showed a slight decrease of mature OLG in the medial CC compared to Cup 
animals, which was not significant ( $p=0.0521$; Two-way ANOVA followed by Sidak's multiple comparisons test). $\mathbf{E}$ The density of mature OLG in the lateral CC was similar in Cuprizone-fed animals with and without EAE induction. Data are shown as mean \pm SEM.

\subsubsection{The density of myelin producing cells and degree of remyelination remain unchanged after immunization with MOG $_{35-55}$ peptide}

Oligodendrocytes are the myelin producing cells, thus any circumstances influencing them could also affect the remyelination capacity. Therefore, LFB-PAS histochemical stainings (Fig. $12 \mathrm{~A}$ ) as well as PLP immunohistochemical stainings and PLP in situ hybridization (Fig. $13 \mathrm{~A}$ ) were performed to evaluate changes in myelin production after EAE induction. A semi-quantitative analysis was performed using the already introduced DM score (table 11) to evaluate the extent of demyelination in the CC.

As shown in 3.1.1, the DM score in the medial and the lateral area of the CC of Cup mice as well as Cup-EAE mice did not differ after 4.5 weeks of Cuprizone feeding (Cup medial: $2.71 \pm 0.29$, Cup-EAE medial: $2.75 \pm 0.16$, Cup lateral: $2.86 \pm 0.14$, Cup-EAE lateral: $3 \pm 0$, Fig. 12 B, C). Five days after Cuprizone withdrawal, the Cup-EAE animals demonstrated a significant decrease in the DM score in the medial (Cup-EAE medial: $1.43 \pm$ 0.37, ${ }^{* *} p<0.01$, Fig. $12 \mathrm{~B}$ ) as well as the lateral CC (Cup-EAE lateral: $2.07 \pm 0.37,{ }^{*} p<0.05$, Fig. $12 \mathrm{C}$ ) compared to the DM time point. The DM score of Cup animals showed a significant decrease in the medial CC (Cup medial: $1.57 \pm 0.3,{ }^{*} p<0.05$, Fig. $12 \mathrm{~B}$ ) but not in the lateral area (Cup lateral: $2.43 \pm 0.23$, Fig. $12 \mathrm{C}$ ). No significant effects were observed between both experimental groups at the RM time point. 


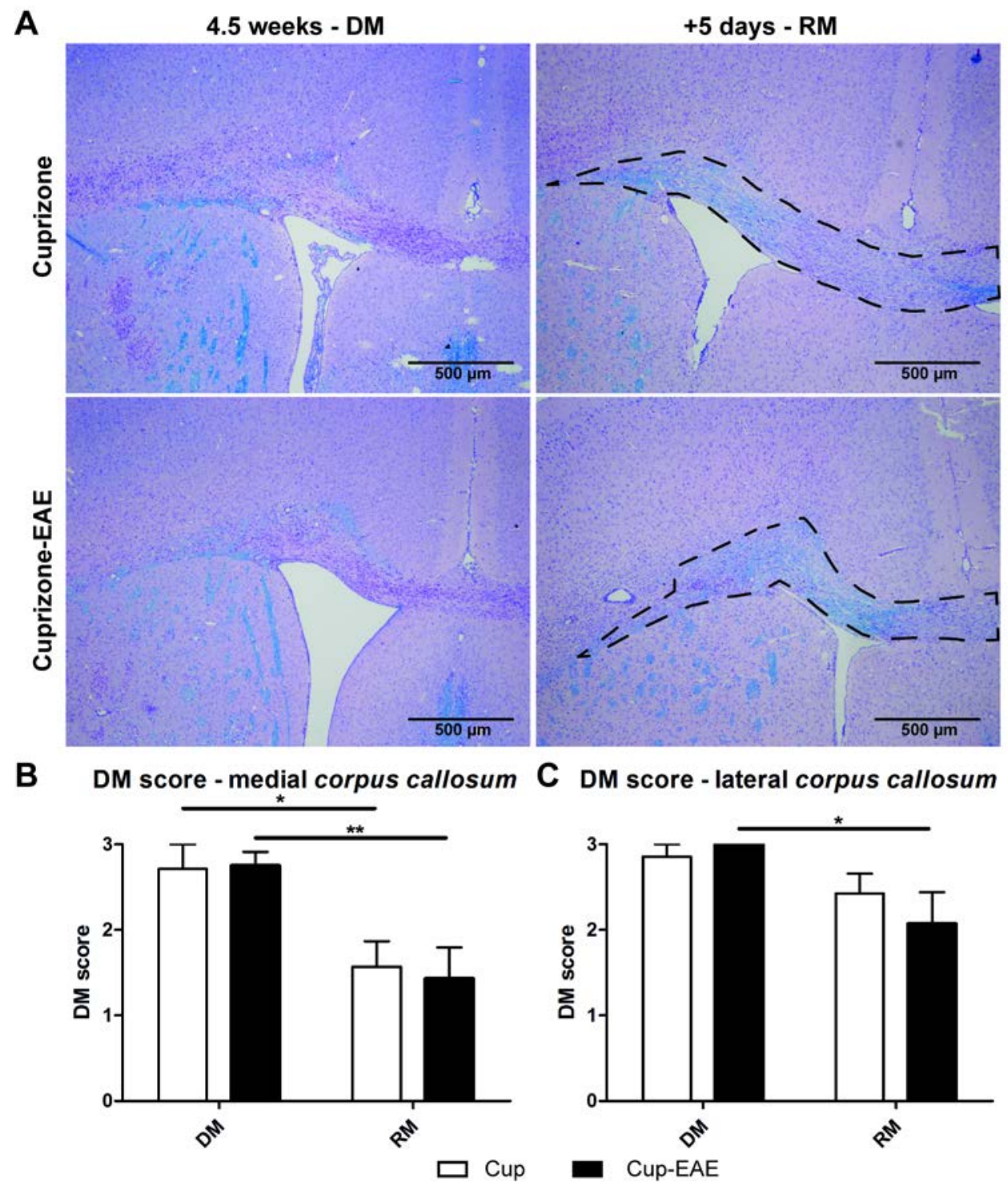

Figure 12: The extent of de- and remyelination is unaffected by EAE induction

A LFB-PAS histochemical stainings of Cup and Cup-EAE mice fed mice at the DM (Cup: $n=7$, CupEAE: $n=7$ ) and RM time points (Cup: $n=7$, Cup-EAE: $n=8$; scale bar: $500 \mu \mathrm{m}$ ). B The remyelination (indicated by dotted lines) slowly takes place in the medial area of the CC (Cup ${ }^{*} p<0.05$, Cup-EAE ${ }^{*} p<0.01$, ( $p=0.0521$; Two-way ANOVA followed by Sidak's multiple comparisons test) and $\mathbf{C}$ the lateral CC (Cup-EAE * $p<0.05$, Two-way ANOVA followed by Sidak's multiple comparisons test) after 5 days on normal chow compared to the DM time point, as demonstrated by semi-quantitative analysis of the CC using a DM score. No significant differences were observed between the experimental groups. Data are shown as mean \pm SEM.

In situ hybridization for PLP-mRNA, one of the major myelin proteins, displays the amount of proteolipid protein mRNA present in the cell, thus serving as an indicator for myelinating OLG. The number of PLP-mRNA ${ }^{+}$cells was evaluated at the RM time point.

The density of PLP-mRNA ${ }^{+}$cells did not change significantly after immunization with MOG $_{35-55}$ peptide neither in the medial (Cup: $668.9 \pm 52.25$ PLP-mRNA ${ }^{+}$cells $/ \mathrm{mm}^{2}$, CupEAE: $517.3 \pm 57.1$ PLP-mRNA ${ }^{+}$cells $/ \mathrm{mm}^{2}$, Fig. $13 \mathrm{~B}$ ) nor in the lateral CC (Cup: $386.8 \pm 74.9$ 
PLP-mRNA ${ }^{+}$cells $/ \mathrm{mm}^{2}$, Cup-EAE: $313.4 \pm 47.91$ PLP-mRNA ${ }^{+}$cells $/ \mathrm{mm}^{2}$, Fig. 13 C). Therefore, the induction of EAE does not seem to influence the PLP-mRNA expression 5 days into remyelination.

A

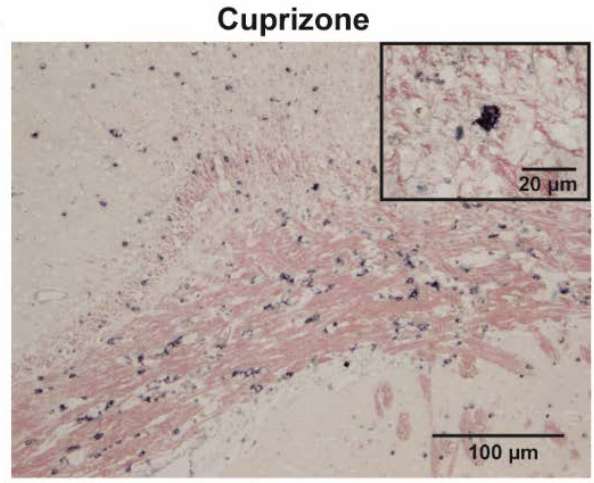

B Myelinating OLG - medial corpus callosum

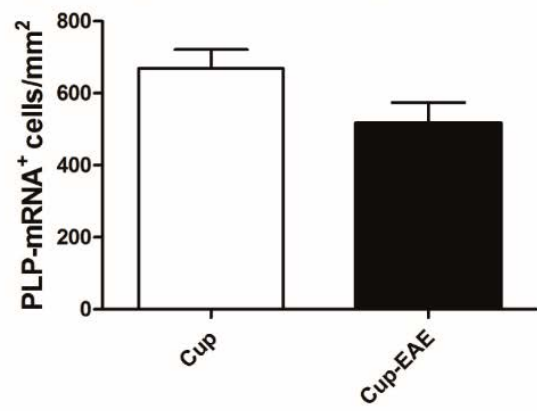

Cuprizone-EAE

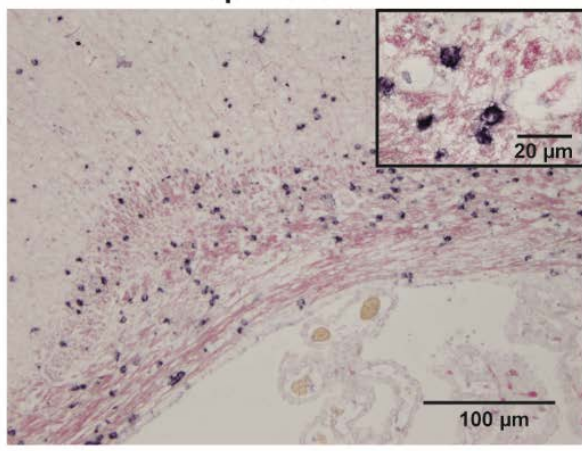

C Myelinating OLG - lateral corpus callosum

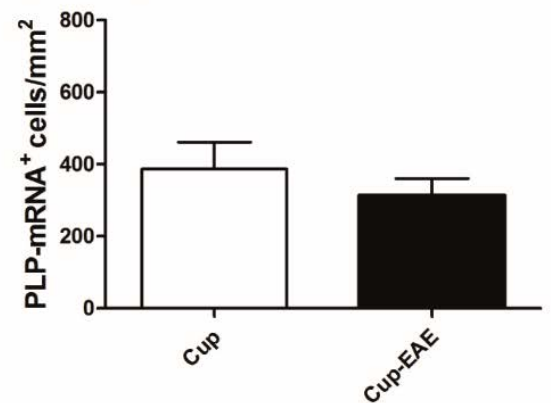

Figure 13: The density of PLP-mRNA ${ }^{+}$cells is not affected by immunization with MOG $_{35-55}$ peptide

A In situ hybridization of PLP-mRNA in Cup $(n=7)$ and Cup-EAE $(n=7)$ mice after five days of RM (scale bar: $100 \mu \mathrm{m}$, inset scale bar: $20 \mu \mathrm{m}$ ). Quantification of PLP-mRNA ${ }^{+}$cells revealed no significant difference between Cuprizone and Cuprizone-EAE animals in B the medial CC and $\mathbf{C}$ the lateral CC (Mann-Whitney test). Data are shown as mean \pm SEM.

\subsubsection{Increased axonal damage in Cuprizone/MOG treated animals}

To verify whether the increased inflammatory milieu would cause an increase of acute axonal damage after 4.5 weeks of Cuprizone administration, brain slices of all three experimental groups were immunohistochemically stained for amyloid precursor protein (APP, Fig. 14 A) which accumulates when axonal transport is disturbed indicating axonal damage. The corpus callosum was again separated into a medial and a lateral area and the amount of $\mathrm{APP}^{+}$axons was quantified for each experimental group. The average density of $\mathrm{APP}^{+}$axons in the medial part of the CC of Cup mice was $133.5 \pm 33.15 \mathrm{APP}^{+}$axons $/ \mathrm{mm}^{2}$, which was similar in Cup-Ova (126.1 $\pm 15.18 \mathrm{APP}^{+}$axons $\left./ \mathrm{mm}^{2}\right)$ and Cup-EAE mice (180.0 \pm $30.99 \mathrm{APP}^{+}$axons $/ \mathrm{mm}^{2}$, Fig. $14 \mathrm{~B}$ ). In contrast, the number of $\mathrm{APP}^{+}$axons in the lateral $\mathrm{CC}$ increased significantly after EAE induction (Cup: $71.66 \pm 16.53 \mathrm{APP}^{+}$axons $/ \mathrm{mm}^{2}$, Cup-EAE: $208.8 \pm 25.242 \mathrm{APP}^{+}$axons $/ \mathrm{mm}^{2},{ }^{\star \star *} p<0.001$, Fig. $14 \mathrm{C}$ ). Furthermore, Cup-EAE mice

$$
\sim 66 \sim
$$


displayed a significant increase of damaged axons in the lateral CC compared to Cup-Ova animals (95.11 $\pm 15.66 \mathrm{APP}^{+}$axons $/ \mathrm{mm}^{2},{ }^{* \star} p<0.01$, Fig. $\left.14 \mathrm{C}\right)$.

A

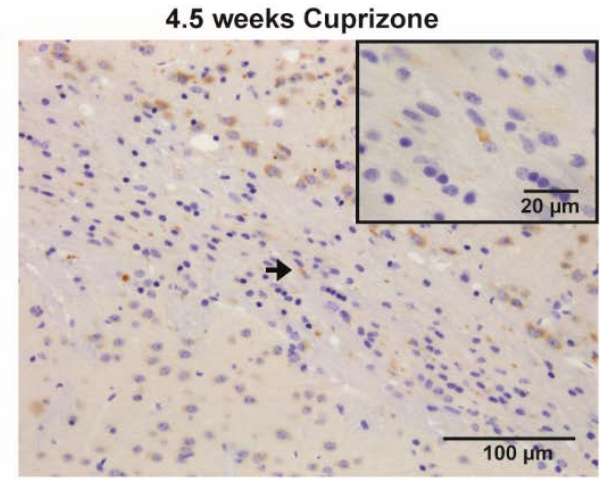

B

APP - medial corpus callosum

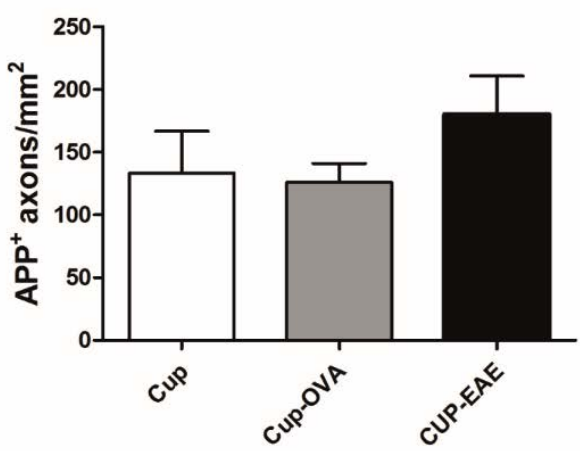

4.5 weeks Cuprizone-EAE

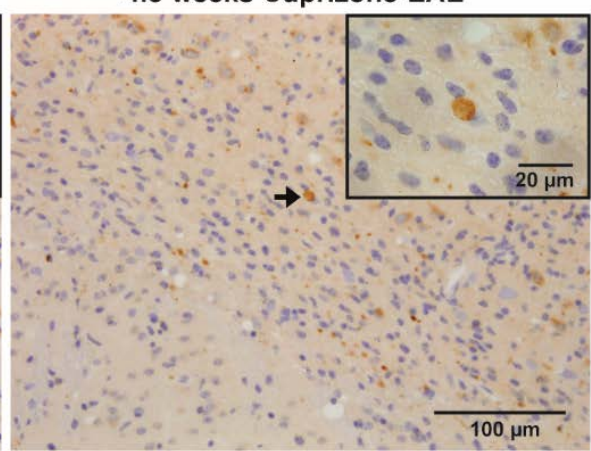

C APP - lateral corpus callosum

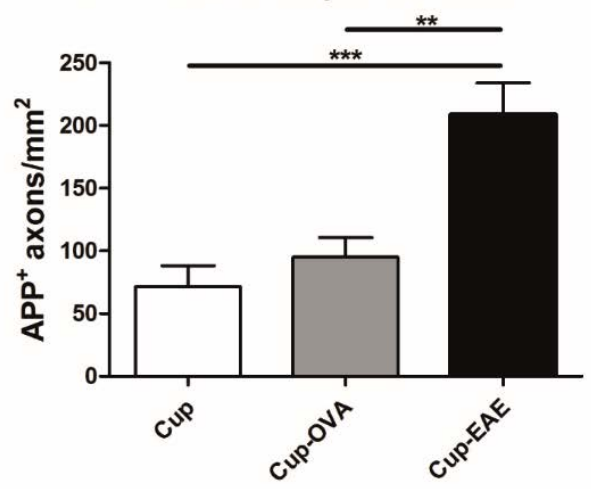

Figure 14: Increased number of $\mathrm{APP}^{+}$axons in the lateral CC of Cup-EAE mice

Quantification of axonal damage by immunohistochemical stainings for APP in the CC of Cup ( $n=6)$, Cup-Ova $(n=6)$ and Cup-EAE $(n=5)$ mice. A Immunohistochemical staining of the CC of Cup animals and Cup-EAE animals for APP (scale bar: $100 \mu \mathrm{m}$, inset scale bar: $20 \mu \mathrm{m}$ ). Areas of axonal damage are marked in brown (arrow). B Quantification of $\mathrm{APP}^{+}$axons in the medial CC revealed no significant difference between the three experimental groups. $\mathrm{C}$ Quantification of $\mathrm{APP}^{+}$axons in the lateral $\mathrm{CC}$ revealed a significant increase in the amount of damaged axons in Cup-EAE mice compared to CupOva $\left({ }^{\star \star} p<0.01\right)$ and Cup mice $\left({ }^{\star * *} p<0.001\right.$, One-way ANOVA). Data are shown as mean \pm SEM.

\subsubsection{The density of myelinated axons is unaffected by EAE induction}

To support the light microscopical data on efficient remyelination in both Cup and Cup-EAE animals on the electron microscopic level, pictures were taken at a 12000 fold magnification and analyzed for the density of myelinated axons in Cuprizone-fed, CuprizoneEAE (Fig. $15 \mathrm{~A}$ ) and naïve control animals. Between four and nine pictures were taken from different areas of the $\mathrm{CC}$ of each mouse, and the amount of myelinated axons was quantified.

Repeated measurements Two-way ANOVA was used to compare all three treatment groups, using the parameters TIME for the DM and RM time point and COND for the different group conditions. A significant interaction was found only for COND $(F(2,19)=8.783$, 
$\left.{ }^{* \star} p<0.01\right)$ and not for TIME $(F(1,19)=3.382, p=0.0816)$, demonstrating a significant difference between the treatment groups and a trend towards an increase in the density of myelinating axons after five days on normal chow compared to the respective DM time point (Fig. 15 B). The former manifested in decreased numbers of myelinated axons in Cup-EAE mice (CupEAE: $0.42 \pm 0.17$ myelinated axons $\left./ \mu m^{2},{ }^{\star *} p<0.01\right)$ compared to naïve animals $(1.27 \pm 0.16$ myelinated axons $/ \mu \mathrm{m}^{2}$ ) after 4.5 weeks of Cuprizone administration, while Cup animals registered a trend towards a decrease in the number of myelinated axons (Cup: $0.67 \pm 0.09$ myelinated axons $/ \mu \mathrm{m}^{2}, p=0.0823$, Fig. $15 \mathrm{~B}$ ). At the RM time point, the density of myelinated fibers in both Cup $\left(0.97 \pm 0.16\right.$ myelinated axons $\left./ \mu m^{2}\right)$ and Cup-EAE animals $(0.82 \pm 0.16$ myelinated axons $/ \mu \mathrm{m}^{2}$, Fig. $15 \mathrm{~B}$ ) recovered to the level of naïve controls, so no significant difference was found between the three experimental groups. No significant difference with regard to the density of myelinated axons was observed at any time point between Cup and Cup-EAE mice. 
A
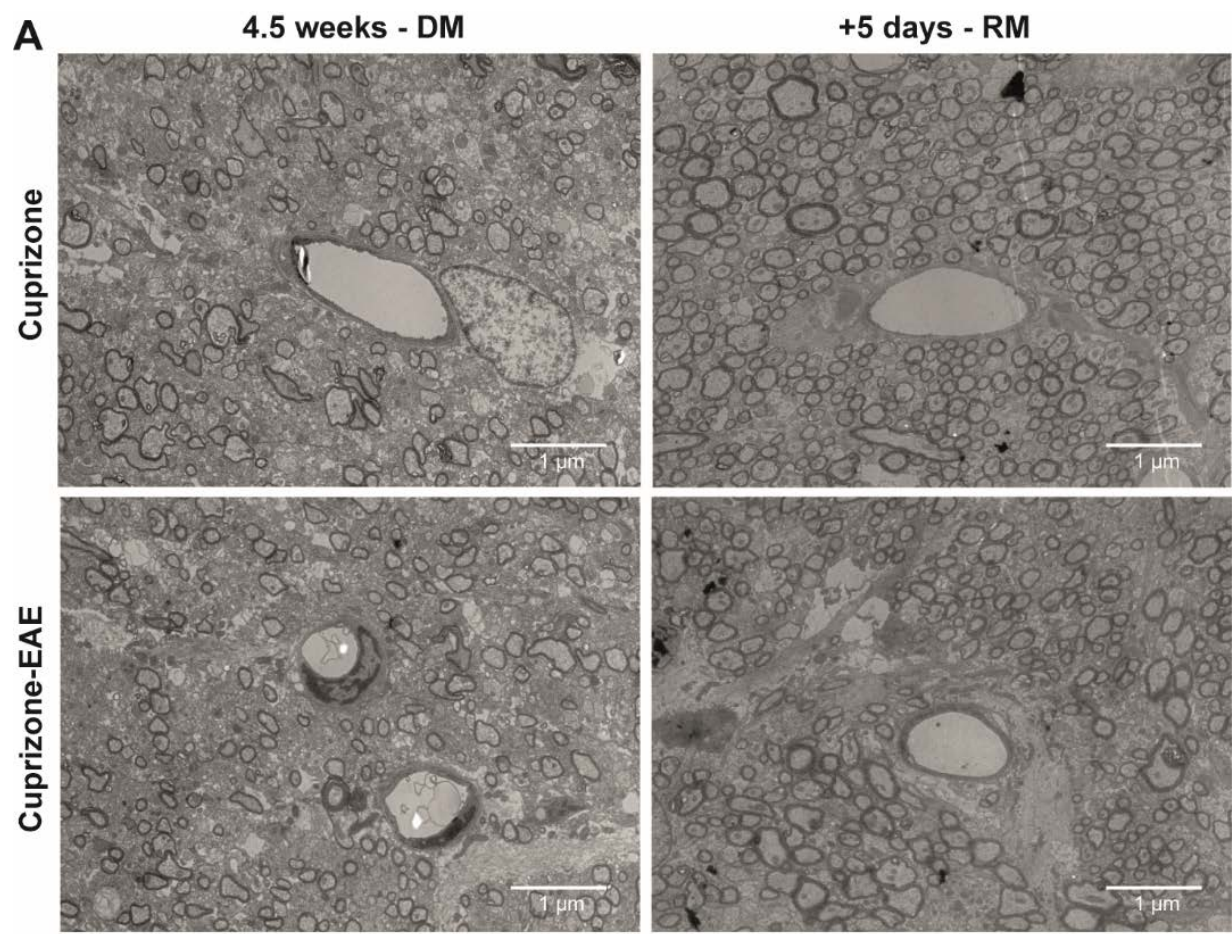

B

Density of myelinated axons

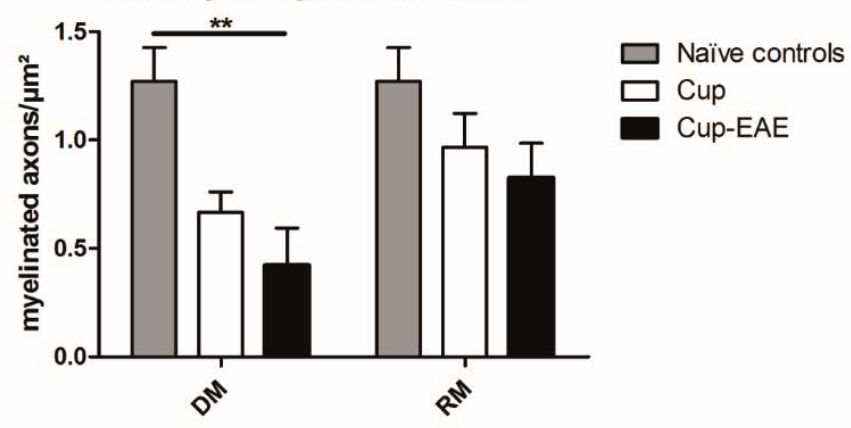

Figure 15: The density of myelinated axons is significantly reduced in Cup-EAE animals at the DM time point and not significantly different between the experimental groups at the RM time point

Quantification of the density of myelinated fibers in the CC of Cuprizone treated animals (DM $n=4$, RM $n=5$ ), Cuprizone-EAE animals (DM $n=3, \operatorname{RM} n=5$ ) and naïve controls ( $n=4)$, evaluated after 4.5 weeks of Cuprizone administration and after five additional days of Cuprizone-free diet. A Electron microscopic pictures of the CC of Cup and Cup-EAE mice at the DM and RM time points (12000 fold magnification, scale bar: $1 \mu \mathrm{m}$ ). B Repeated measures Two-way ANOVA revealed a trend toward an increase in myelinated fibers between the DM and RM time points $(F(1,19)=3.382, p=0.082)$ and a significant interaction between the experimental groups $\left(F(2,19)=8.783,{ }^{* \star} p<0.01\right)$. The density of myelinated axons was significantly decreased in Cup-EAE animals $\left({ }^{*} p<0.01\right)$ compared to naïve controls at the DM time point, while Cup animals showed a tendency toward a decrease $(p=0.082)$. No significant effects were registered after 5 days on normal chow for neither experimental group compared to naïve controls. No changes were observed between Cuprizone and Cuprizone-EAE animals. Data are shown as mean \pm SEM. 


\section{Part II - Results}

Influence of transcranial alternating current stimulation on spontaneous remyelination in in vitro and in vivo models 


\subsection{Influence of transcranial alternating current stimulation on spontaneous remyelination in in vitro and in vivo models}

In the following part, the effect of electrical stimulation (ESTIM) on spontaneous (re-) myelination was evaluated in different in vitro and in vivo models. Primary rat oligodendroglial cell cultures as well as primary mixed cortical cell cultures consisting of oligodendrocytes, astrocytes and neurons were created to investigate the influence of ESTIM on oligodendroglial survival, myelin production and myelination of unmyelinated axons. To evaluate possible effects of ESTIM on remyelination in rodent models of demyelination, transcranial alternating currents were applied to rats with focally demyelinated lesions in the gray matter as well as to mice which showed a delayed remyelination of the corpus callosum after prolonged administration of Cuprizone.

\subsubsection{Influence of alternating current stimulation on cell viability and myelin production in primary oligodendroglial cell cultures and mixed cortical cell cultures}

Primary oligodendrocyte cultures were used to evaluate the effect of a $10 \mathrm{~mA}$ electrical field fluctuating in a $10 \mathrm{~Hz}$ frequency on cell viability and myelin production in the absence of axonal fibers. Myelinating mixed cortical cultures consisting of oligodendrocytes and cortical neurons were used to evaluate the effect of electrical stimulation on cytotoxicity and myelination of axonal fibers.

\subsubsection{The amount of myelin producing oligodendrocytes in primary oligodendrocyte cultures remains unchanged after electrical stimulation}

To investigate the effect of electrical stimulation on oligodendrocyte growth, differentiation and myelin production, primary oligodendrocyte cultures were cultivated and stimulated for four consecutive days (ESTIM). The amount of oligodendroglial $\mathrm{O}^{+}$cells expression MBP was determined by immunocytochemistry (Fig. $16 \mathrm{~A}, \mathrm{~B}$ ) and compared to unstimulated control cultures (NOSTIM).

The number of $\mathrm{O} 4$ positive cells in ESTIM cultures was normalized to the number of O4 positive cells in NOSTIM cultures. No change in the number of O4 positive cells of ESTIM cultures was observed neither over the time course of four days nor in relation to NOSTIM cultures (ESTIM d1: $166.8 \pm 45.38 \%$ of NOSTIM, ESTIM d2: $109.9 \pm 13.81 \%$ of NOSTIM, ESTIM d3: $128.5 \pm 13.18 \%$ of NOSTIM, ESTIM d4: $106.6 \pm 15.0 \%$ of NOSTIM, Fig. 16 A).

A Two-way ANOVA was conducted to examine the number of $\mathrm{O}^{+} \mathrm{MBP}^{+}$cells in NOSTIM and ESTIM cultures over the time course of 4 days and a significant increase over time was observed $\left({ }^{* \star *} p<0.0001, F(3,60)=16.54\right) .4 .81 \pm 3.28 \%$ of all DAPI positive cells in the unstimulated group showed a co-labeling for $\mathrm{O} 4$ and MBP at day 1 , which increased over 
time (NOSTIM d2: $21.22 \pm 4.14 \%$, NOSTIM d3: $21.16 \pm 3.43 \%$, NOSTIM d4: $20.0 \pm 3.49 \%$, Fig. 16 B). Similarly, the amount of O4/MBP double positive cells of the stimulated cultures increased over time as well (ESTIM d1: $2.02 \pm 1.06 \%$, ESTIM d2: $14.19 \pm 3.43 \%$, ESTIM d3: $23.8 \pm 5.28 \%$, ESTIM d4: $22.45 \pm 3.75 \%$, Fig. 16 B). Nevertheless, no significant effect was observed at any time point by comparing both treatment groups.

\section{A $\quad \mathrm{O4}^{+}$cells in ESTIM cultures}

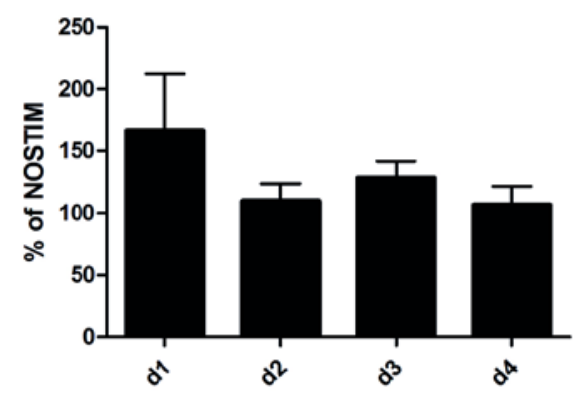

B $\quad \mathrm{O4}^{+}$cells expressing MBP

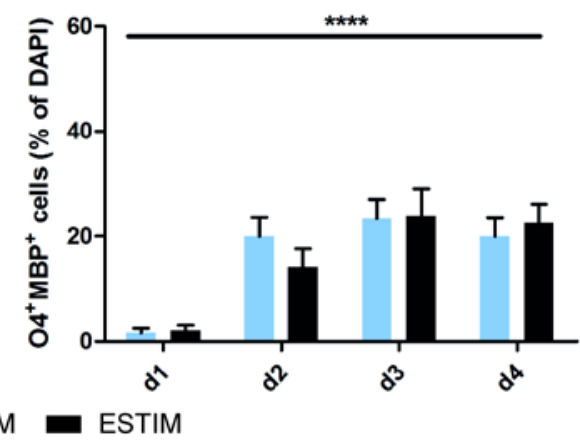

Figure 16: $\mathrm{O4}^{+}$oligodendrocytes and expression of MBP are unaffected by electrical stimulation

Quantification of immunocytochemical stainings for O4 and MBP of OLG cultures after 1, 2, 3 and 4 days of either ESTIM ( $n=6-10)$ or NOSTIM $(n=6-10)$. A The amount of $4^{+}$cells in the ESTIM cultures, normalized to NOSTIM cultures, remained stable over the time course of four days. B The number of myelin forming OLG increased significantly over time ${ }^{\star \star \star *} p<0.0001, F(3,60)=16.54$, Two-way ANOVA). No significant differences were observed between NOSTIM and ESTIM cultures at any time point. Data are shown as mean \pm SEM.

\subsubsection{Myelination and cell viability in mixed cortical cell cultures are unaffected by electrical stimulation}

The effect of electrical stimulation on the myelination capacity of oligodendrocytes in the presence of axonal fibers was evaluated using mixed cortical cultures consisting of oligodendrocytes, astrocytes and cortical neurons. Immunocytochemistry for neurofilament NF200 and MBP were performed to visualize myelinated and non-myelinated axons (Fig. 17 A). To quantify the number of myelinated fibers, five visual fields were randomly determined on each cover slip, and axonal fibers were counted using a counting grid (Fig. 17 B). 
A
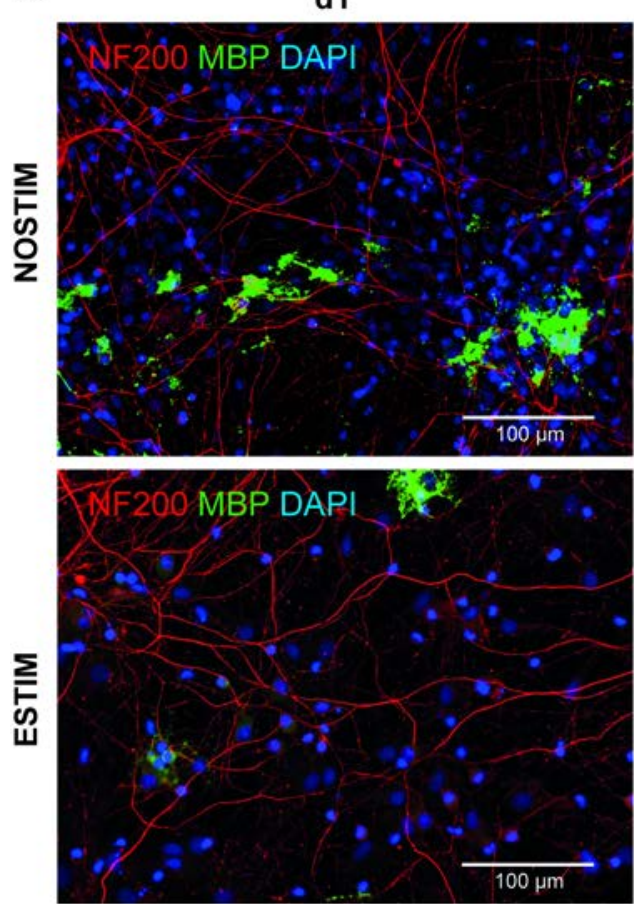

B

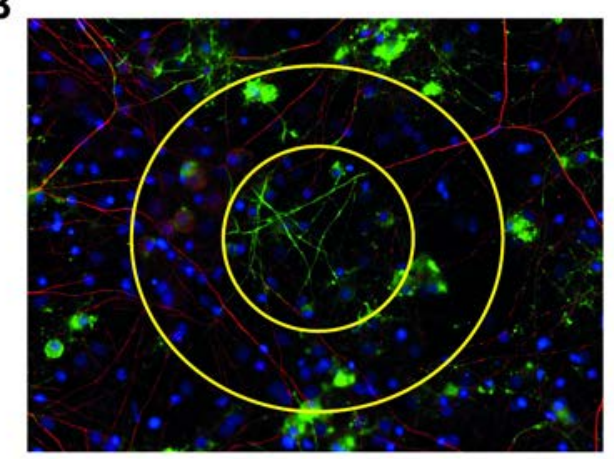

d4
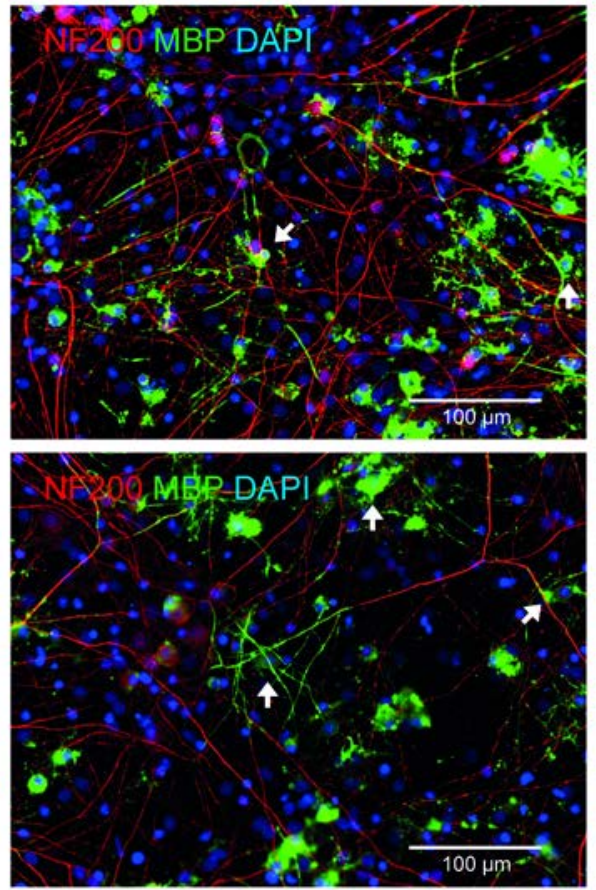

C

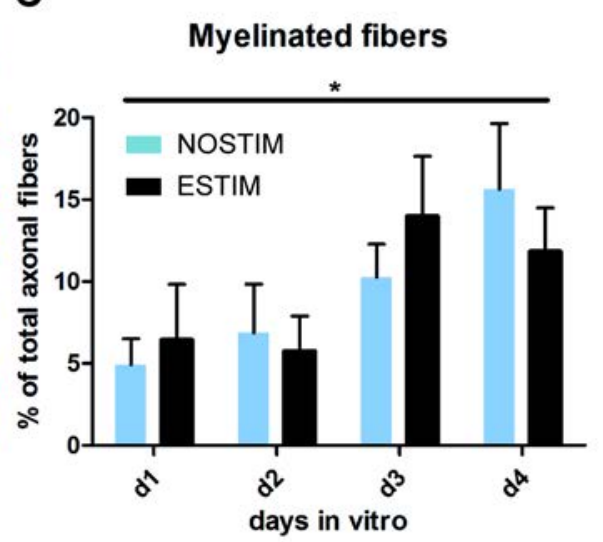

Figure 17: ESTIM does not influence the amount of myelinated fibers

Immunocytochemical stainings for NF200 (red) and MBP (green) were performed for mixed cortical cultures after 1, 2, 3 and 4 days of either NOSTIM ( $n=8-10)$ or ESTIM (n=8-10). A One day after the OLG cultures had been transferred onto neuronal cultures, mostly non-myelinated axons (NF200 ${ }^{+}$MBP) were found. At d4, however, several MBP ${ }^{+}$OLG were observed in both experimental groups, enwrapping nearby axons with myelin sheaths (arrows). B The number of myelinated and non-myelinated fibers was determined using a counting grid consisting of two circles overlying the image. Only axonal fibers crossing a yellow line were counted. C Percentage of myelinated axonal fibers in relation to the total amount of axonal fibers of both treatment groups after up to 4 days in vitro. A Two-way ANOVA analyzing the percentage of myelinated axons over the time course of four days for both experimental groups revealed a significant increase over time $\left({ }^{*} p<0.05, F(3,66)=3.762\right.$, Twoway ANOVA). Sidak's multiple comparisons test demonstrated no significant differences between the two groups. Data are shown as mean \pm SEM. 
The analysis of the mean amount of myelinated fibers expressed as the percentage of the total amount of fibers by Two-way ANOVA revealed a significant increase in myelinated fibers over time (NOSTIM d1: $4.92 \pm 1.59 \%$, ESTIM d1: $6.43 \pm 3.39 \%$, NOSTIM d2: $6.88 \pm$ 2.96\%, ESTIM d2: $5.74 \pm 2.15 \%$, NOSTIM d3: $10.27 \pm 2.0 \%$, ESTIM d3: $13.97 \pm 3.67 \%$, NOSTIM d4: $15.63 \pm 4.0 \%$, ESTIM d4: $11.83 \pm 2.67 \%$, ${ }^{*} p<0.05, F(3,66)=3.762$, Fig. $\left.17 \mathrm{C}\right)$. However, no effect was observed at any time point by comparing both experimental groups.

Experiments in OLG cultures demonstrated that the total amount of Olig2 ${ }^{+}$OLG was not affected by ESTIM. Therefore, we wanted to know if the cell viability after electrical stimulation remained unaffected in mixed cortical cultures as well. By performing a LDH cytotoxicity test, the level of cytotoxicity was determined after either NOSTIM or ESTIM treatments over the time course of four days (Fig. 18). Both groups depicted a cytotoxicity of around 50-70\% (NOSTIM d1: $61.01 \pm 23.89 \%$, ESTIM d1: $49.44 \pm 20.77 \%$; NOSTIM d2: $75.84 \pm 19.33 \%$, ESTIM d2: $64.19 \pm 17.54 \%$; NOSTIM d3: $41.50 \pm 18.50 \%$, ESTIM d3: 42.26 \pm 17.08\%; NOSTIM d4: $50.62 \pm 21.86 \%$, ESTIM d4: $50.77 \pm 22.03 \%$; Fig. 18), which did not change after four days. Furthermore, ESTIM appeared to have no impact on cell viability compared to NOSTIM at any time point.

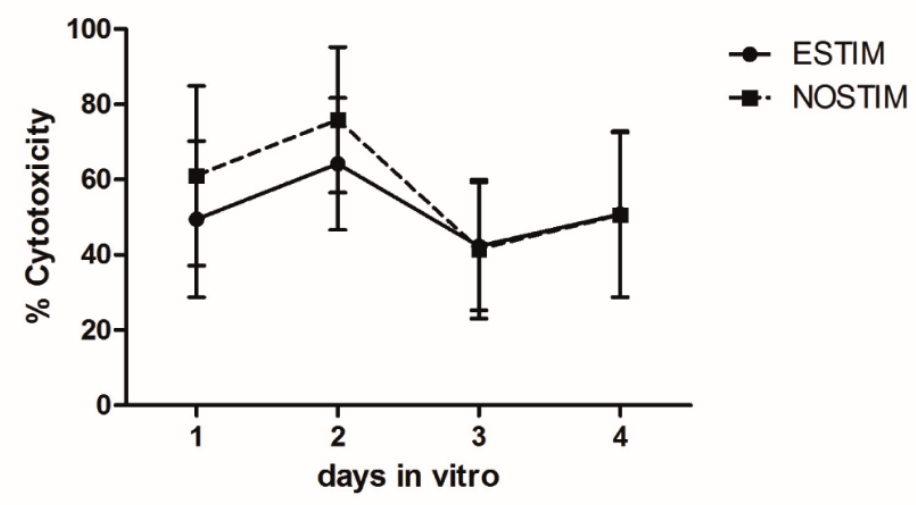

Figure 18: Cell survival is not influenced by electrical stimulation

Evaluation of cell viability after 1 (NOSTIM $n=5, \operatorname{ESTIM} n=5), 2$ (NOSTIM $n=5$, ESTIM $n=5$ ), 3 (NOSTIM $n=6$, ESTIM $n=6$ ) and 4 (NOSTIM $n=6$, ESTIM $n=6$ ) days of ESTIM or NOSTIM treatment by a LDH cytotoxicity assay. The level of cytotoxicity remains unchanged after four days of ESTIM compared to NOSTIM. No effect was observed over the time course of four days for either NOSTIM or ESTIM (Two-way ANOVA followed by Sidak's multiple comparisons test). Data are shown as mean \pm SEM. 


\subsubsection{Influence of transcranial alternating current stimulation on spontaneous remyelination in rodent models of demyelination}

\subsubsection{Focal EAE model}

The stereotactical injection of the myelin specific antibody Z2 in combination with mouse complement leads to focal demyelinated lesions in the cortex of Lewis rats. tACS (ESTIM, $100 \mu \mathrm{A}, 10 \mathrm{~Hz}$ ) and sham stimulation (sham) were applied on three consecutive days for 10 minutes each day during the initial phase of lesion formation. Animals were sacrificed at $3,5,7,10,14$ and 21 days post injection (d.p.i.), and the extent of the demyelinated area as well as the presence of different oligodendrocyte populations was quantified on immunohistochemically stained brain sections. A third experimental group, consisting of rats which were neither electrically nor sham stimulated but injected with the same Z2/mouse complement solution (NOSTIM), was also assessed to compare the extent of the MBP depleted area.

\subsection{Intracortical injection of anti-MOG antibodies (Z2) and mouse complement leads to demyelinated lesions devoid of MBP with minimal axonal loss injection}

Following the stereotactical injection of $\mathrm{Z} 2$ antibodies and mouse complement, a cone-shaped area devoid of MBP was observed surrounding the injection site in the primary motor cortex and extending subpially along the somatosensory cortex towards the visceral area (Fig. 19). No axonal loss was detected in the demyelinated area, while only minimal axonal loss was observed at the site of the stereotactical injection, confirmed by Bielschowsky silver impregnation (Fig. 19). Whereas after five days a pronounced demyelinated lesion was found following ESTIM as well as sham treatment, MBP immunohistochemistry indicated a fast spontaneous remyelination, starting after seven days (detected by thin MBP positive signals in the previously demyelinated area) and leading to an almost completely remyelinated lesion at 21 d.p.i. for both treatment groups (Fig. 20). 

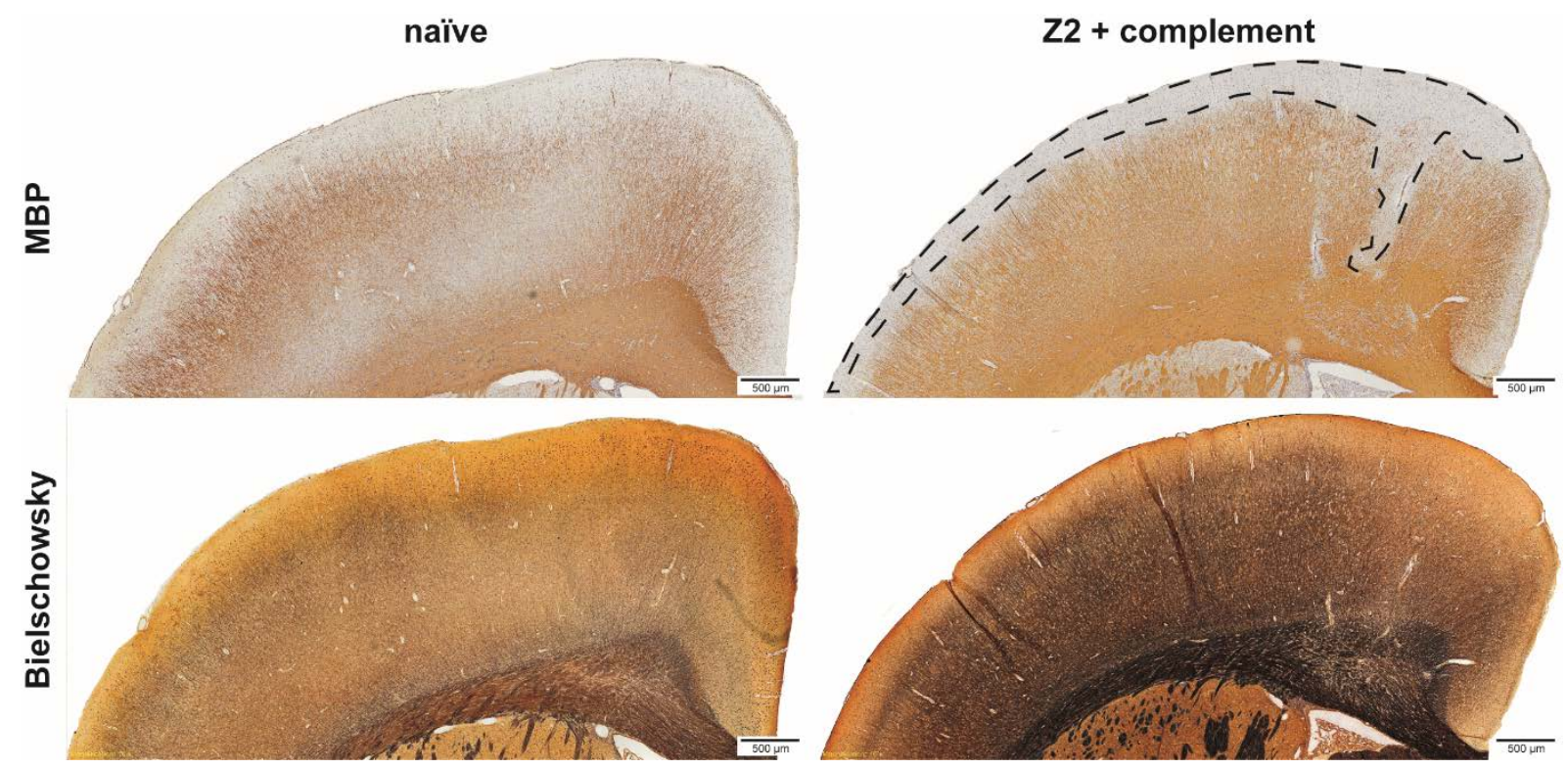

Figure 19: Formation of a demyelinated lesion after stereotactical injection with $Z 2$ antibody and mouse complement without substantial axonal damage and loss

Depicted are brain hemispheres from a naïve rat and a rat injected with the MOG-specific antibody Z2 in combination with mouse complement. The hemispheres were stained for MBP to detect loss of myelin and silver impregnated with Bielschowsky's technique to exclude important axonal damage and loss (scale bar: $500 \mu \mathrm{m}$ ). After injection of $Z 2$ and mouse complement, a demyelinated lesion was detected in the motor cortex around the injection site, which extended subpially along the somatosensory cortex towards the visceral area (dotted line). Despite the loss of MBP signal, little or no axonal damage was observed by Bielschowsky silver impregnation, indicating primary demyelination. 


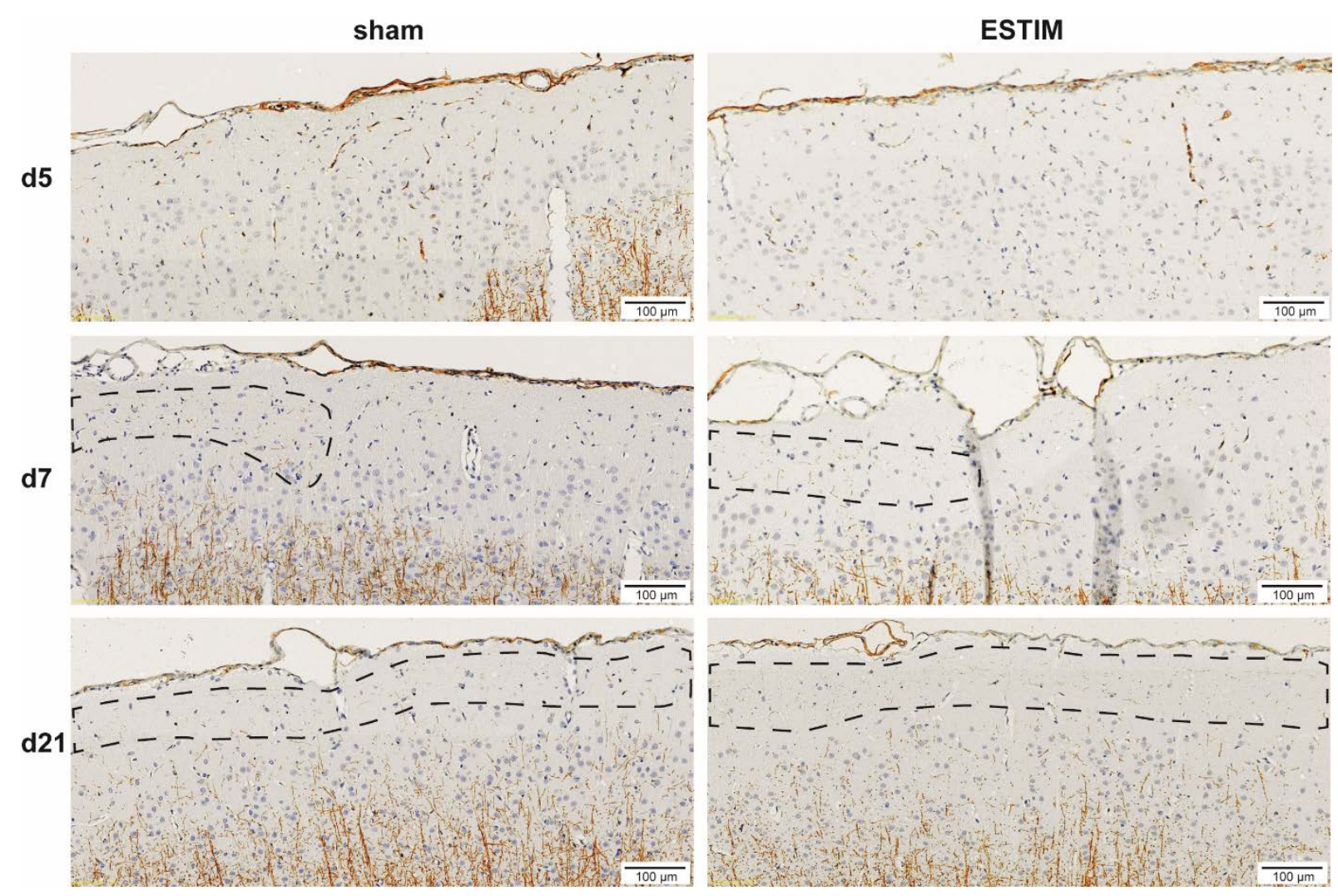

Figure 20: Lesion formation of sham and ESTIM animals over a time course of 21 days demonstrated by MBP immunohistochemistry

Close view of the subpial area of sham and ESTIM treated animals after 5, 7 and 21 days, immunostained for MBP (scale bar: $100 \mu \mathrm{m}$ ). At d5, the subpial area of both sham and ESTIM animals depicted a loss of MBP signal. The first signs of remyelination were detected at $d 7$ in both treatment groups, indicated by thin MBP positive signals (dotted lines), which extended across the entire subpial area after 21 days.

\subsection{Remyelination of the MBP depleted area is slightly impaired at three weeks following tACS and sham stimulation}

The extent of the demyelinated area (DM area) was analyzed by measuring the MBP depleted areas in the gray matter and subpial area on MBP immuno-stained brain sections. The size of the MBP depleted area decreased significantly over the time course of 21 days for all three experimental groups (NOSTIM: ${ }^{* \star \star \star} p<0.0001$, sham: ${ }^{*} p<0.05$, ESTIM: ${ }^{\star \star} p<0.01$, Fig. 21). The demyelinated area was largest at three days after lesion induction (NOSTIM: $1.62 \pm 0.42 \mathrm{~mm}^{2}$, sham: $1.64 \pm 0.22 \mathrm{~mm}^{2}$, ESTIM: $1.34 \pm 0.24 \mathrm{~mm}^{2}$ ), gradually decreased after five (NOSTIM: $0.87 \pm 0.2 \mathrm{~mm}^{2}$, sham: $1.03 \pm 0.2 \mathrm{~mm}^{2}$, ESTIM: $1.86 \pm 0.23 \mathrm{~mm}^{2}$ ), seven (NOSTIM: $0.79 \pm 0.26 \mathrm{~mm}^{2}$, sham: $1.25 \pm 0.27 \mathrm{~mm}^{2}$, ESTIM: $1.62 \pm 0.29 \mathrm{~mm}^{2}$ ) and ten days (NOSTIM: $0.32 \pm 0.07 \mathrm{~mm}^{2}$, sham: $0.51 \pm 0.24 \mathrm{~mm}^{2}$, ESTIM: $0.61 \pm 0.2 \mathrm{~mm}^{2}$ ) and almost disappeared after 14 (NOSTIM: $0.08 \pm 0.02 \mathrm{~mm}^{2}$, sham: $0.67 \pm 0.26 \mathrm{~mm}^{2}$, ESTIM: $0.46 \pm$ $0.13 \mathrm{~mm}^{2}$ ) and 21 days (NOSTIM: $0.03 \pm 0.02 \mathrm{~mm}^{2}$, sham: $0.58 \pm 0.16 \mathrm{~mm}^{2}$, ESTIM: $0.59 \pm$ $0.13 \mathrm{~mm}^{2}$, Fig. 21) for all three experimental groups. However, the ESTIM group showed a 
trend toward a larger DM area size at 5 d.p.i. compared to sham and a significant increase compared to NOSTIM at 5 and 7 d.p.i. (ESTIM vs. sham d5: $p=0.056$, ESTIM vs. NOSTIM d5: ${ }^{* \star} p<0.01$, ESTIM vs. sham d7: $p=0.454$, ESTIM vs. NOSTIM d7: ${ }^{\star} p<0.05$ ), which leveled out to the DM area size of the sham group after 10 days. Furthermore, sham, in contrast to ESTIM, demonstrated a trend towards a larger DM area after 14 days compared to NOSTIM (sham vs. NOSTIM d14: $p=0.082$, Fig. 21). No significant differences in the size of the DM area were observed between sham and ESTIM animals at any time point.

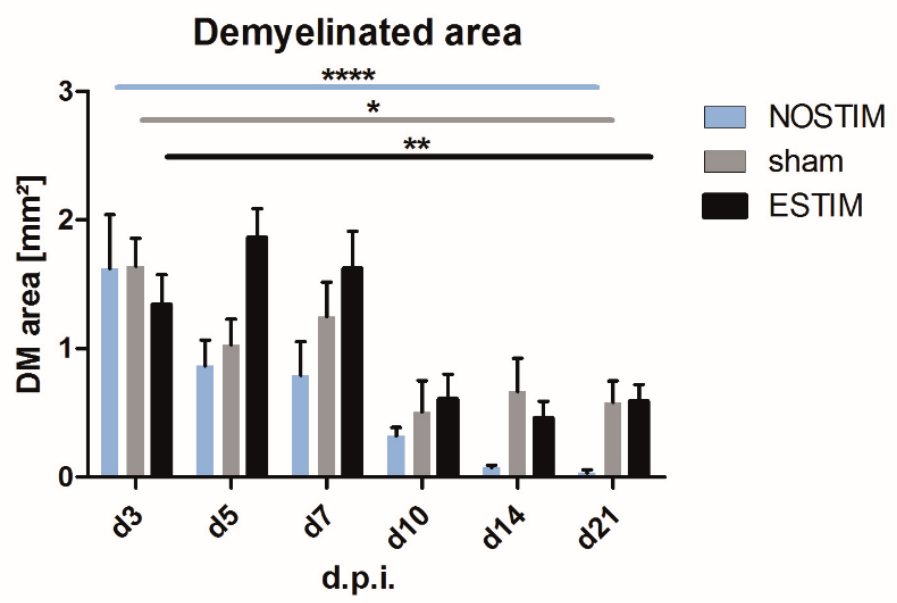

Figure 21: Demyelinated lesion size gradually decreases over a time course of three weeks

Quantification of the demyelinated area after lesion induction reveals a gradual decrease in lesion size within 21 days (NOSTIM: ${ }^{* * * *} p<0.0001, n=7-11$; sham: ${ }^{*} p<0.05, n=4-6$; ESTIM: ${ }^{* *} p<0.01, n=4-8$ ). No significant effect was observed by comparing sham and ESTIM animals. The size of the DM area of ESTIM animals was significantly larger after $5\left({ }^{\star \star} p<0.01\right)$ and $7\left({ }^{\star} p<0.05\right)$ days compared to NOSTIM animals. DM area was determined by analyzing MBP immunohistochemical stainings. Data were analyzed by Two-way ANOVA followed by Tukey's multiple comparisons test to compare all three experimental groups and by Kruskal-Wallis test followed by Dunn's Multiple Comparison Test to analyze the lesion formation over time for each group separately. Data are shown as mean \pm SEM.

\subsection{The density of oligodendrocyte precursor cells and mature oligodendrocytes is unchanged after ESTIM}

Oligodendrocyte precursor cells are mandatory for efficient remyelination. For this reason, the amount of OPC and mature OLG in the gray matter of either sham treated or ESTIM treated Lewis rats after induction of cortical demyelinated lesions was quantified. The extracellular milieu inside and around the demyelinated lesion can be different from the healthy tissue, thus affecting OLG populations in a different way. Therefore, four separate counting areas were defined to evaluate the different OLG populations (Fig. 22 A): demyelinated lesion area (DM lesion, 3-14 d.p.i.), perilesional area (perilesion, 3 and 5 d.p.i.), remyelinated area (RM area, 7-21 d.p.i.) and normal appearing gray matter (NAGM). Since only very few animals displayed a demyelinated lesion after 21 days, no statistical 
analysis could be performed for the demyelinated lesion area at 21 d.p.i., and the time point was removed for this specific counting area. To determine the density of OPC and mature OLG, respectively, immunohistochemistry for the transcription factor Olig2 and the myelin protein TPPP/p25 were used (Fig. 22 A). Cells lacking the marker for mature OLG $\left(\mathrm{Olig}^{+} \mathrm{p} 25^{-}\right)$were considered OPC. Antibodies against MBP were used to label myelin, thus visualizing de- and remyelination. DAPI was used to label the cell nuclei.

Three days after lesion induction, $78.78 \pm 31.89$ Olig2 ${ }^{+} \mathrm{p} 25^{-}$cells $/ \mathrm{mm}^{2}$ were found inside the demyelinated lesion of sham treated animals and $100.7 \pm 4.82$ Olig2 $^{+} \mathrm{p} 25^{-}$ cells $/ \mathrm{mm}^{2}$ were found in ESTIM treated animals (Fig. 22 B). At 5 d.p.i., the amount of OPC in the ESTIM group decreased to $55.43 \pm 17.14$ Olig2 ${ }^{+} \mathrm{p} 25^{-}$cells $/ \mathrm{mm}^{2}$, although not significantly, whereas the OPC number in the sham group remained unchanged $\left(116.2 \pm 7.92\right.$ Olig2 $^{+}$p25 cell/mm², Fig. 22A). Similarly, no significant effects on OPC numbers were observed for the remaining time points (ESTIM d7: $87.4 \pm 24.51$ Olig2 $^{+}$p25 cell/mm², sham d7: $106.7 \pm 37.86$ Olig2 ${ }^{+}$p25 cells $/ \mathrm{mm}^{2}$, ESTIM d10: $48.07 \pm 11.67$ Olig2 $^{+}$p25 cells $/ \mathrm{mm}^{2}$, sham d10: $49.11 \pm$ 4.17 Olig2 ${ }^{+}{ }^{+} 25^{-}$cells $/ \mathrm{mm}^{2}$, ESTIM d14: $47.97 \pm 16.34$ Olig2 $^{+}{ }^{\circ} 25^{-}$cells $/ \mathrm{mm}^{2}$, sham d14: 51.72 \pm 3.84 Olig $^{+} \mathrm{p} 25^{-}$cells $/ \mathrm{mm}^{2}$, Fig. 22 B). However, Two-way ANOVA revealed a significant change of OPC over time $\left({ }^{*} p<0.05, F(4,29)=3.500\right)$. The density of $p 25^{+}$, mature OLG inside the demyelinated area did not differ between sham and ESTIM but, similar to OPC, demonstrated a decrease over the time course of 14 days $\left({ }^{*} p<0.05, F(4,28)=3.144\right.$, Fig. 22

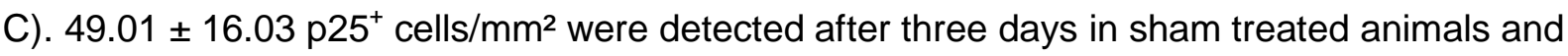
$56.78 \pm 6.07 \mathrm{p} 25^{+}$cells $/ \mathrm{mm}^{2}$ in tACS treated animals. Almost the same number of mature OLG was found after five (sham: $56.7 \pm 10.9$ p25 cells $/ \mathrm{mm}^{2}$, ESTIM: $51.25 \pm 7.79$ p25 cells $/ \mathrm{mm}^{2}$ ), seven (sham: $32.37 \pm 4.58 \mathrm{p} 25^{+}$cells $/ \mathrm{mm}^{2}$, ESTIM: $48.89 \pm 13.14 \mathrm{p} 25^{+}$ cells $/ \mathrm{mm}^{2}$ ), ten days (sham: $27.07 \pm 11.07 \mathrm{p} 25^{+}$cells $/ \mathrm{mm}^{2}$, ESTIM: $32.62 \pm 4.7 \mathrm{p} 25^{+}$ cells $/ \mathrm{mm}^{2}$ ) and 14 days (sham: $28.25 \pm 6.99 \mathrm{p} 25^{+}$cells $/ \mathrm{mm}^{2}$, ESTIM: $22.85 \pm 6.39$ p25 cells $/ \mathrm{mm}^{2}$, Fig. $22 \mathrm{C}$ ).

The time points 3 and 5 d.p.i. represented an early stage in which no remyelination of the demyelinated area was detected yet. Therefore, a perilesional area was defined to evaluate OLG populations which were resident in the lesion periphery. The total number of p25 $5^{+}$OLG in the perilesional area of both experimental groups was higher than the number of p25 $5^{+}$OLG in the DM lesion (sham: ${ }^{*} p<0.05$, ESTIM: ${ }^{*} p<0.01$ ) but smaller than the $p 25^{+}$OLG population in the NAGM (sham: ${ }^{* \star} p<0.01$, ESTIM: ${ }^{*} p<0.05$ ). In contrast to the ESTIM group, only the total Olig2 $^{+} \mathrm{p} 25^{-}$OPC population in the perilesional area of sham animals demonstrated a significantly reduced cell count compared to NAGM (sham: ${ }^{\star \star} p<0.01$ ). Besides that, no further effect was observed between the experimental groups in regard to OPC (sham d3: $62.66 \pm 17.45$ Olig2 $^{+}$p25 cells $/ \mathrm{mm}^{2}$, ESTIM d3: $93.43 \pm 4.825$ Olig2 $^{+} \mathrm{p} 25^{-}$ cells $/ \mathrm{mm}^{2}, p=0.35$; sham d5: $102.7 \pm 11.26$ Olig2 $^{+} \mathrm{p} 25^{-}$cells $/ \mathrm{mm}^{2}$, ESTIM d5: $79.86 \pm 20.44$ 
Olig2 ${ }^{+} 2^{-}$cells $/ \mathrm{mm}^{2}$, Fig. $\left.22 \mathrm{D}\right)$ in the perilesional area. While mature OLG in the perilesional area showed similar numbers with and without ESTIM (sham d3: $102 \pm 22.07$ p25 cells $/ \mathrm{mm}^{2}$, ESTIM d3: $122.2 \pm 15.3$ p25 cells $/ \mathrm{mm}^{2}$, sham d5: $145.3 \pm 10.24$ p25 cells $/ \mathrm{mm}^{2}$, ESTIM d5: $152.6 \pm 16.4 \mathrm{p} 25^{+}$cells $/ \mathrm{mm}^{2}$, Fig. $22 \mathrm{E}$ ), a significant increase in p25 cells was registered from d3 to d5 ( $\left.{ }^{*} p<0.05, F(1,9)=5.434\right)$. 
A

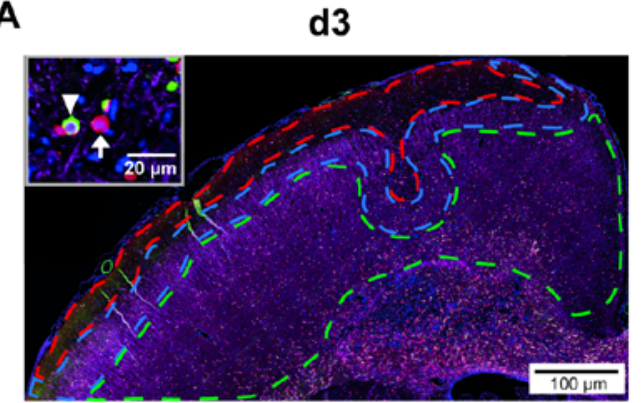

- - - DM lesion $\quad-\quad-\quad$ peri lesion

B

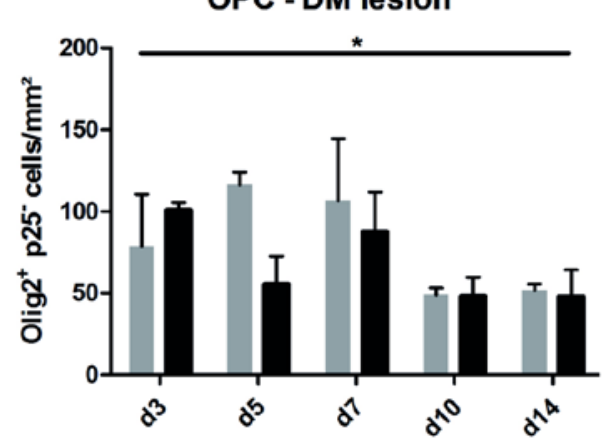

D

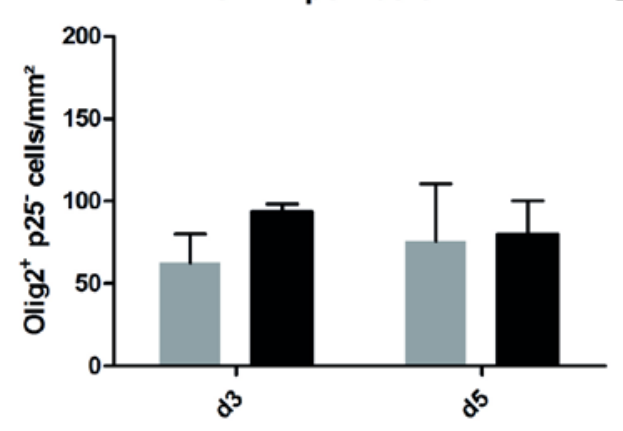

$\mathbf{F}$

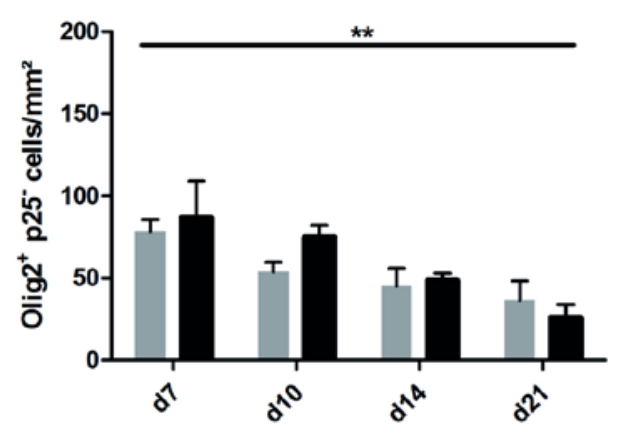

H

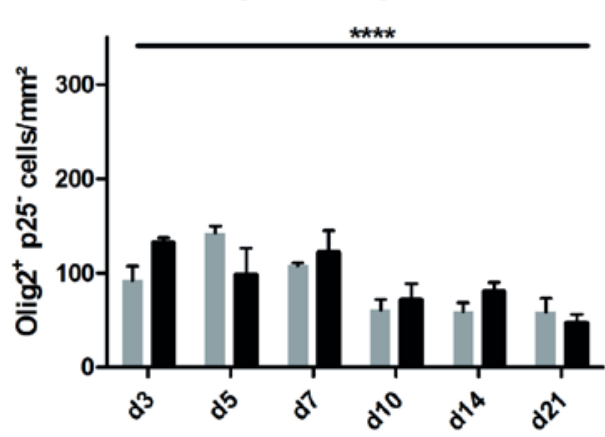

d14

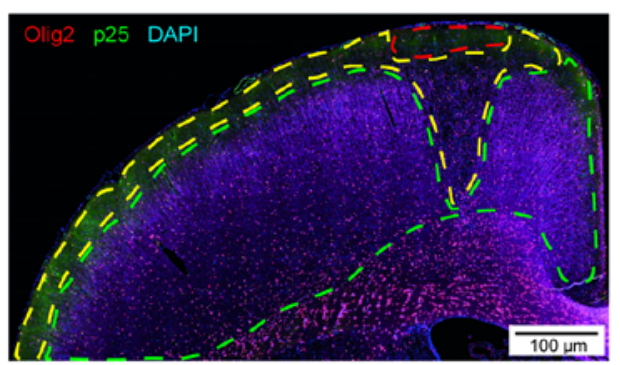

C

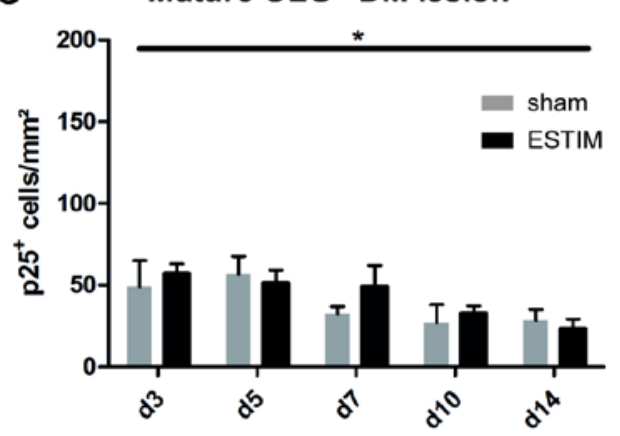

E mature OLG - perilesion

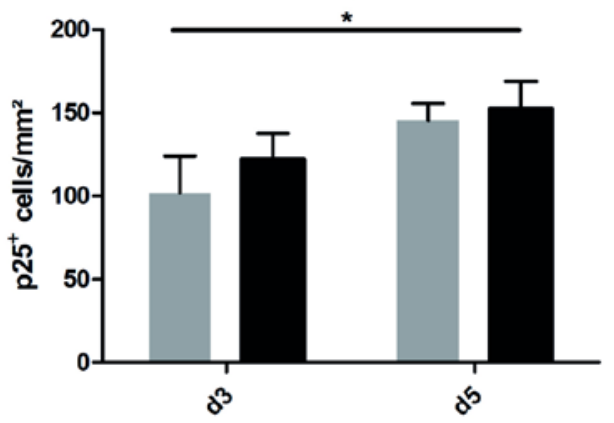

G mature OLG - RM area

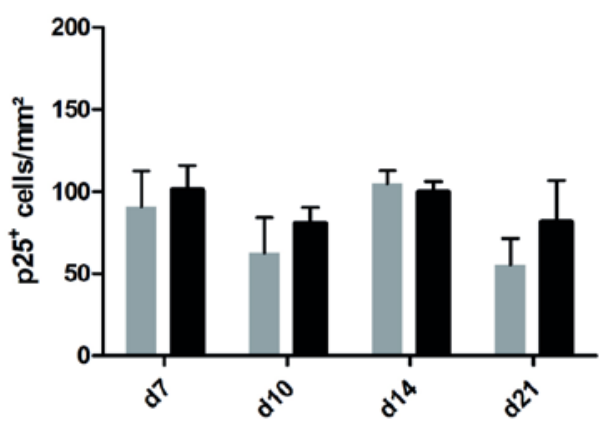

Mature OLG - NAGM

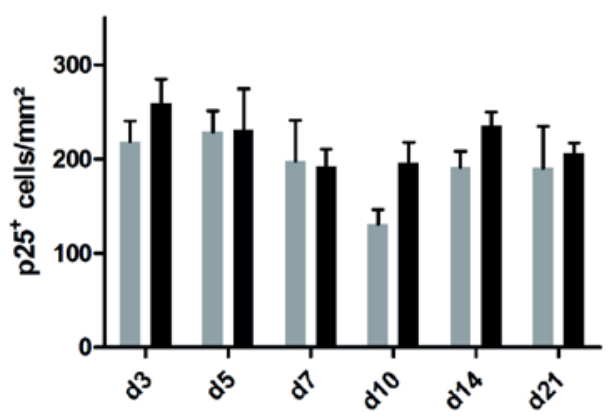

$\sim 81 \sim$ 
Figure 22: Oligodendrocyte populations are largely unaffected by ESTIM (continued from previous page)

A Immunohistochemical stainings with antibodies against Olig2 (arrow) and p25 (arrow head) were performed to quantify OPC and mature OLG populations after either sham or ESTIM treatment in four different areas: inside the lesion, in the perilesional area, in the remyelinated area and in the NAGM. The density of B OPC inside the lesion was not effected by ESTIM, while Two-way ANOVA revealed a significant decrease over the time course $\left({ }^{*} p<0.05, F(4,29)=3.500\right.$; sham $n=3-5$, ESTIM $\left.n=3-5\right)$. C The density of mature OLG (sham $n=3-5$, ESTIM $n=3-5$ ) inside the lesion remained unchanged after tACS. Two-way ANOVA analysis demonstrated a significant drop in the number of mature OLG over time $\left({ }^{*} p<0.05, F(4,28)=3.144\right)$. D The number of OPC (sham $n=3-4$, ESTIM $n=3$ ) in the perilesional area demonstrated no effect between both experimental groups nor over the time course of two days, $\mathbf{E}$ while the mature OLG (sham $n=3-4$, ESTIM $n=3$ ) in the perilesional area showed no effect between ESTIM and sham animals as well, but demonstrated a significant increase in $\mathrm{p}^{2} 5^{+}$cell numbers from $\mathrm{d} 3$ to $\mathrm{d} 5$ ( $\left.{ }^{*} p<0.05, F(1,9)=5.434\right)$. F A significant decrease in the OPC population was observed in the $\mathrm{RM}$ area $\left({ }^{\star \star} p<0.01, F(3,27)=6.046\right)$, while again no change was observed between sham $(n=3-5)$ and ESTIM ( $n=3-5)$ animals. G The number of mature OLG in the RM area remained unchanged for sham $(n=3-5)$ as well as ESTIM animals $(n=3-5)$. H The OPC population in the NAGM decreased significantly over time $\left.{ }^{* * * \star} p<0.0001, F(5,37)=7.224\right)$, whereas no differences between ESTIM $(n=3-5)$ and sham ( $n=2-5)$ animals was observed at any time point. I Neither sham $(n=2-5)$ nor ESTIM $(n=3-5)$ animals demonstrated a significant effect on mature OLG in the NAGM. Data were analyzed by Twoway ANOVA, followed by Sidak's multiple comparisons test and are presented as mean \pm SEM.

Since after seven days the first signs of remyelination were observed, a RM area was defined for the time points 7, 10, 14 and 21 d.p.i., covering previously demyelinated areas which showed signs of remyelination, e.g. thin and discontinuous $\mathrm{MBP}^{+}$myelin fibers. $\mathrm{A}$ significant decrease in the amount of OPC was observed by analysis of the RM area of sham and ESTIM animals over the time course of two weeks with Two-way ANOVA (ESTIM d7: $87.05 \pm 22.11$ Olig2 $^{+}$p25 cells $/ \mathrm{mm}^{2}$, ESTIM d10: $75.07 \pm 7.07$ Olig2 $^{+}$p25 cells $/ \mathrm{mm}^{2}$, ESTIM d14: $48.75 \pm 4.2$ Olig2 $^{+} \mathrm{p} 25^{-}$cells $/ \mathrm{mm}^{2}$, ESTIM d21: $25.86 \pm 8.04$ Olig2 $^{+} \mathrm{p} 25^{-}$cells $/ \mathrm{mm}^{2}$; sham d7: $78.32 \pm 7.27$ Olig2 ${ }^{+} \mathrm{p} 25^{-}$cells $/ \mathrm{mm}^{2}$, sham d10: $53.72 \pm 5.79$ Olig2 ${ }^{+} \mathrm{p} 25^{-}$cells $/ \mathrm{mm}^{2}$, sham d14: $45.15 \pm 10.76$ Olig2 $^{+}{ }^{+} 25^{-}$cells $/ \mathrm{mm}^{2}$, sham d21: $36.25 \pm 11.99$ Olig2 $^{+} \mathrm{p} 25^{-}$cells $/ \mathrm{mm}^{2}$; ${ }^{* *} p<0.01, F(3,27)=6.046$; Fig. $\left.22 \mathrm{~F}\right)$. The number of mature $\mathrm{p} 25^{+} \mathrm{OLG}$, however, remained stable (ESTIM d7: $101.1 \pm 14.86 \mathrm{p} 25^{+}$cells $/ \mathrm{mm}^{2}$, ESTIM d10: $80.76 \pm 9.45 \mathrm{p} 25^{+}$cells $/ \mathrm{mm}^{2}$, ESTIM d14: $99.81 \pm 6.2$ p25 cells $/ \mathrm{mm}^{2}$, ESTIM d21: $81.56 \pm 25.19$ p25 cells $/ \mathrm{mm}^{2}$; sham d7: $90.86 \pm 21.66$ p25 cells $/ \mathrm{mm}^{2}$, sham d10: $62.65 \pm 21.51$ p25 cells $/ \mathrm{mm}^{2}$, sham d14: $105.1 \pm$ 7.64 p $25^{+}$cells $/ \mathrm{mm}^{2}$, sham d21: $55.42 \pm 15.93$ p25 cells $/ \mathrm{mm}^{2}$; Fig. $22 \mathrm{G}$ ).

Regarding the NAGM, a Two-way ANOVA revealed a significant decrease in the OPC population over the time course of 21 days (ESTIM d3: $132.5 \pm 4.65$ Olig2 ${ }^{+}$p25 cells $/ \mathrm{mm}^{2}$, ESTIM d5: $98.0 \pm 28.1$ Olig2 $^{+}{ }^{+} 2^{-}$cells $/ \mathrm{mm}^{2}$, ESTIM d7: $122.0 \pm 22.89$ Olig2 $^{+}{ }^{\mathrm{p}} 25^{-}$cells $/ \mathrm{mm}^{2}$, ESTIM d10: $71.7 \pm 17.06$ Olig2 $^{+} \mathrm{p} 25^{-}$cells $/ \mathrm{mm}^{2}$, ESTIM d14: $80.64 \pm 9.62$ Olig2 $^{+} \mathrm{p} 25^{-}$ 
cells $/ \mathrm{mm}^{2}$, ESTIM d21: $46.91 \pm 9.31$ Olig2 $^{+}{ }^{\mathrm{p}} 25^{-}$cells $/ \mathrm{mm}^{2}$; sham d3: $92.33 \pm 14.75$ Olig2 ${ }^{+}$p25 cells $/ \mathrm{mm}^{2}$, sham d5: $142.5 \pm 7.55$ Olig2 $^{+}$p25 cells $/ \mathrm{mm}^{2}$, sham d7: $108.1 \pm 2.54$ Olig2 ${ }^{+}$p25 cells $/ \mathrm{mm}^{2}$, sham d10: $83.19 \pm 28.21$ Olig2 $^{+}{ }^{+} 25^{-}$cells $/ \mathrm{mm}^{2}$, sham d14: $59.31 \pm$ 9.11 Olig2 $^{+} \mathrm{p} 25^{-}$cells $/ \mathrm{mm}^{2}$, sham d21: $58.66 \pm 14.39$ Olig2 $^{+} \mathrm{p} 25^{-}$cells $/ \mathrm{mm}^{2} ;{ }^{\star \star \star \star} p<0.0001$, $F(5,37)=7.224$; Fig. $22 \mathrm{H}$ ), while Sidak's multiple comparisons test revealed no significant effect between sham and ESTIM animals. The amount of $\mathrm{p}^{+} 5^{+}$OLG in the NAGM demonstrated no effect neither for the sham group (sham d3: $218.4 \pm 22.01 \mathrm{p} 25^{+}$cells $/ \mathrm{mm}^{2}$, sham d5: $229.1 \pm 22.26$ p25 cells $/ \mathrm{mm}^{2}$, sham d7: $197.8 \pm 43.09$ p25 cells $/ \mathrm{mm}^{2}$, sham d10: $146.9 \pm 55.93$ p25 cells $/ \mathrm{mm}^{2}$, sham d14: $191.2 \pm 16.91$ p25 ${ }^{+}$cells $/ \mathrm{mm}^{2}$, sham d21: $190.6 \pm$

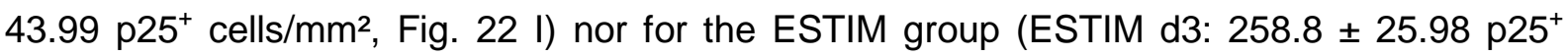
cells $/ \mathrm{mm}^{2}$, ESTIM d5: $230.5 \pm 44.24 \mathrm{p} 25^{+}$cells $/ \mathrm{mm}^{2}$, ESTIM d7: $192.0 \pm 18.35 \mathrm{p} 25^{+}$ cells $/ \mathrm{mm}^{2}$, ESTIM d10: $196.0 \pm 21.68$ p25 cells $/ \mathrm{mm}^{2}$, ESTIM d14: $235.6 \pm 14.62$ p25 cells/mm², ESTIM d21: $206.0 \pm 11.06$ p25 cells $/ \mathrm{mm}^{2}$, Fig. 22 I).

\subsection{Proliferation of oligodendrocyte precursor cells and mature oligodendrocytes is unaffected by tACS}

Bromodeoxyuridin (BrdU) was i.p.-injected into the rats immediately after induction of focal cortical demyelination and then continuously every 12 hours for 5 days to label proliferating cells in the tissue. By combining BrdU with the OLG marker Oilg2, we were able to evaluate the effect of tACS on OLG proliferation in defined brain areas (DM lesion, perilesional area, RM area and NAGM).

$6.89 \pm 1.01 \mathrm{Olig}^{+} \mathrm{BrdU}^{+}$cells $/ \mathrm{mm}^{2}$ were found inside the demyelinated area of sham animals after three days, and $11.11 \pm 1.13 \mathrm{Olig}^{+} \mathrm{BrdU}^{+}$cells $/ \mathrm{mm}^{2}$ in ESTIM animals (Fig. 23 A). The number of proliferating OLG remained unaffected after ESTIM treatment and did not change significantly over the time course of 14 days (sham d5: $14.17 \pm 5.87$ Olig2 $^{+} \mathrm{BrdU}^{+}$ cells $/ \mathrm{mm}^{2}$, ESTIM d5: $18.81 \pm 5.23$ Olig2 ${ }^{+} \mathrm{BrdU}^{+}$cells $/ \mathrm{mm}^{2}$, sham d7: $9.56 \pm 1.97 \mathrm{Olig}^{+} \mathrm{BrdU}^{+}$ cells $/ \mathrm{mm}^{2}$, ESTIM d7: $10.85 \pm 1.58$ Olig2 $^{+} \mathrm{BrdU}^{+}$cells $/ \mathrm{mm}^{2}$, sham d10: $11.83 \pm 2.72$ Olig2 ${ }^{+} \mathrm{BrdU}^{+}$cells $/ \mathrm{mm}^{2}$, ESTIM d10: $9.92 \pm 3.93 \mathrm{Olig}^{+} \mathrm{BrdU}^{+}$cells $/ \mathrm{mm}^{2}$, sham d14: $20.77 \pm$ 9.75 Olig2 ${ }^{+} \mathrm{BrdU}^{+}$cells $/ \mathrm{mm}^{2}$, ESTIM d14: $11.39 \pm 5.02 \mathrm{Olig}^{+} \mathrm{BrdU}^{+}$cells $/ \mathrm{mm}^{2}$, Fig. $23 \mathrm{~A}$ ).

With $3.46 \pm 0.57 \mathrm{Olig}^{+} \mathrm{BrdU}^{+}$cells $/ \mathrm{mm}^{2}$ in the sham group and $2.9 \pm 2.9 \mathrm{Olig}^{+} \mathrm{BrdU}^{+}$ cells $/ \mathrm{mm}^{2}$ in the ESTIM group at 3 d.p.i. as well as $4.53 \pm 1.78 \mathrm{Olig}^{+} \mathrm{BrdU}^{+}$cells $/ \mathrm{mm}^{2}$ in the sham group and $6.53 \pm 3.11 \mathrm{Olig}^{+} \mathrm{BrdU}^{+}$cells $/ \mathrm{mm}^{2}$ in the ESTIM group at 5 d.p.i., the density of OLG that had proliferated during the respective time frame in the perilesional area did not differ significantly from the demyelinated lesion. No significant difference in BrdU positive OLG between the two experimental groups was found.

Compared to the NAGM, the density of $\mathrm{BrdU}^{+}$OLG in the RM area of sham animals was significantly increased (sham ${ }^{*} p<0.05$, statistic not shown in Fig. 23). $11.86 \pm 4.49$ 
Olig2 ${ }^{+} \mathrm{BrdU}^{+}$cells $/ \mathrm{mm}^{2}$ were found in the RM area of ESTIM animals after seven days, which remained stable over time (ESTIM d10: $9.08 \pm 2.64 \mathrm{Olig}^{+} \mathrm{BrdU}^{+}$cells $/ \mathrm{mm}^{2}$, ESTIM d14: 7.09 \pm 3.22 Olig2 $^{+} \mathrm{BrdU}^{+}$cells $/ \mathrm{mm}^{2}$, ESTIM d21: $5.05 \pm 1.71 \mathrm{Olig}^{+} \mathrm{BrdU}^{+}$cells $/ \mathrm{mm}^{2}$, Fig. $23 \mathrm{C}$ ) . Similarly, the number of proliferating OLG in the RM area of sham treated animals did not change over the time course either (sham d7: $12.65 \pm 6.65 \mathrm{Olig}^{+} \mathrm{BrdU}^{+}$cells $/ \mathrm{mm}^{2}$, sham d10: $5.42 \pm 1.7$ Olig2 $^{+} \mathrm{BrdU}^{+}$cells $/ \mathrm{mm}^{2}$, sham d14: $14.65 \pm 2.62 \mathrm{Olig}^{+} \mathrm{BrdU}^{+}$cells $/ \mathrm{mm}^{2}$, sham d21: $15.84 \pm 3.74$ Olig2 $^{+} \mathrm{BrdU}^{+}$cells $/ \mathrm{mm}^{2}$, Fig. $23 \mathrm{C}$ ).

Three days after lesion induction, $2.13 \pm 0.83 \mathrm{Olig}^{+} \mathrm{BrdU}^{+}$cells $/ \mathrm{mm}^{2}$ were found in the NAGM of sham treated animals and $3.08 \pm 1.56 \mathrm{Olig}^{+} \mathrm{BrdU}^{+}$cells $/ \mathrm{mm}^{2}$ of ESTIM treated animals (Fig. $23 \mathrm{D}$ ). The number of proliferating OLG in both experimental groups remained stable over time (sham d5: $4.25 \pm 1.3 \mathrm{Olig}^{+} \mathrm{BrdU}^{+}$cells $/ \mathrm{mm}^{2}$, sham d7: $3.68 \pm 1.4$ Olig2 ${ }^{+} \mathrm{BrdU}^{+}$cells $/ \mathrm{mm}^{2}$, sham d10: $2.16 \pm 0.48 \mathrm{Olig}^{+} \mathrm{BrdU}^{+}$cells $/ \mathrm{mm}^{2}$, sham d14: $9.04 \pm 1.02$ Olig2 ${ }^{+} \mathrm{BrdU}^{+}$cells $/ \mathrm{mm}^{2}$, sham d21: $7.49 \pm 2.05$ Olig2 $^{+} \mathrm{BrdU}^{+}$cells $/ \mathrm{mm}^{2}$; ESTIM d5: $6.24 \pm 3.14$ Olig2 ${ }^{+} \mathrm{BrdU}^{+}$cells $/ \mathrm{mm}^{2}$, ESTIM d7: $4.33 \pm 0.81 \mathrm{Olig}^{+} \mathrm{BrdU}^{+}$cells $/ \mathrm{mm}^{2}$, ESTIM d10: $8.33 \pm 1.1$ Olig2 ${ }^{+} \mathrm{BrdU}^{+}$cells $/ \mathrm{mm}^{2}$, ESTIM d14: $4.6 \pm 1.98 \mathrm{Olig}^{+} \mathrm{BrdU}^{+}$cells $/ \mathrm{mm}^{2}$, ESTIM d21: $4.54 \pm$ $2.61 \mathrm{Olig}^{+} \mathrm{BrdU}^{+}$cells $/ \mathrm{mm}^{2}$, Fig. $23 \mathrm{D}$ ). However, a trend was found if comparing both experimental groups at 10 d.p.i. $(p=0.056)$, demonstrating a minor increase in the Olig2 ${ }^{+} \mathrm{BrdU}^{+}$cells in the ESTIM group. 

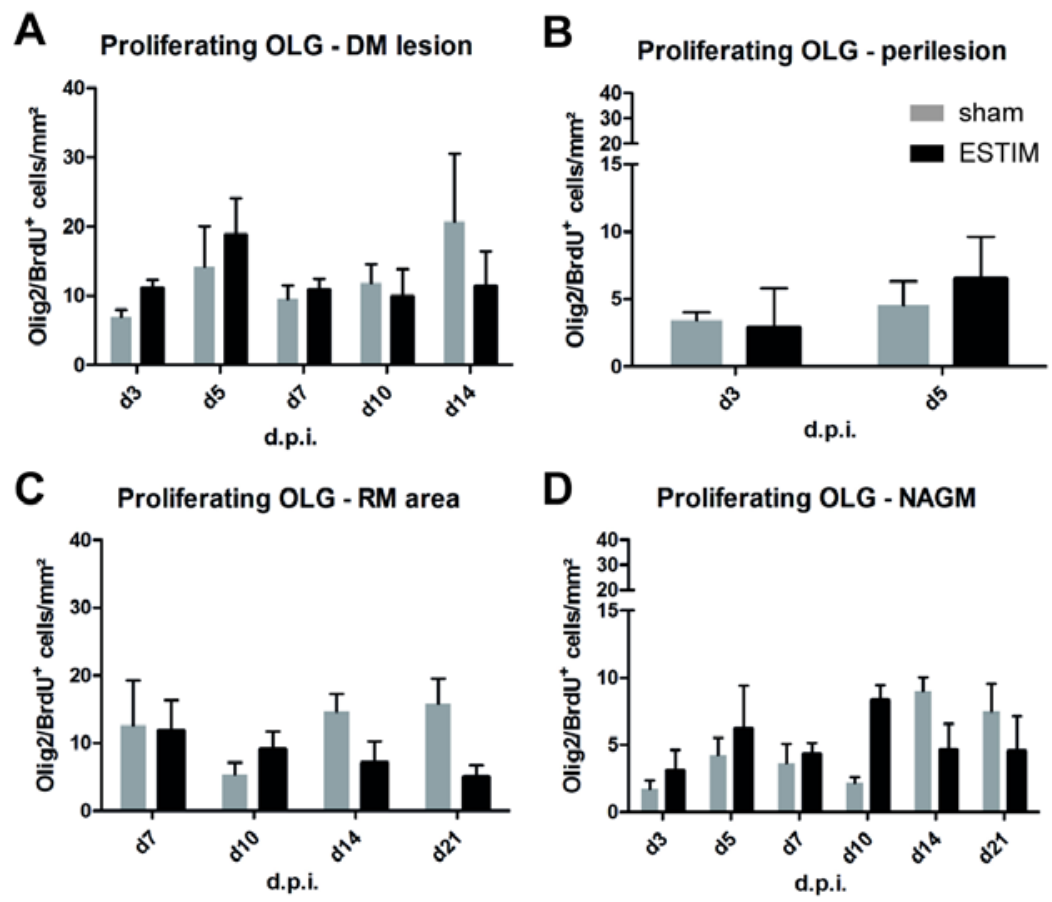

Figure 23: Numbers of proliferating OLG are unaltered after ESTIM

Quantification of proliferating OLG in the DM lesion, the perilesion, the RM area and the NAGM of sham and ESTIM treated rats by immunohistochemical stainings against BrdU and Olig2. Cell counts of proliferating OLG in A the DM lesion (sham $n=2-3$, ESTIM $n=3-5$ ), B the perilesional area (sham $n=2-3$, ESTIM $n=3$ ), C the RM area (sham $n=4$, ESTIM $n=4$ ) and D the NAGM (sham $n=3-4$, ESTIM $n=3-5$ ) yielded no significant effect after electrical stimulation, as indicated by Two-way ANOVA followed by Sidak's multiple comparisons test. Data are shown as mean \pm SEM.

\subsubsection{Chronic Cuprizone model}

The chronic Cuprizone model (continuous Cuprizone administration for 13 weeks) was used to induce a toxic demyelination with impaired remyelination in the $\mathrm{CC}$ of $\mathrm{BI} / \mathrm{J} / \mathrm{J}$ mice. Transcranial alternating currents were applied to modulate the remyelination of the demyelinated area by targeting OPC and mature OLG. The animals were separated into four groups and stimulated for either five days (5d ESTIM) or ten days (10d ESTIM). Sham stimulated animals served as controls ( $5 \mathrm{~d}$ sham, $10 \mathrm{~d}$ sham). The medial and lateral parts of the CC were analyzed separately with regard to the different OLG populations and the extent of remyelination.

\subsection{DM score unaffected by either $5 \mathrm{~d}$ or $10 \mathrm{~d}$ ESTIM}

In contrast to the commonly used Cuprizone model (5 weeks of Cuprizone feeding) which demonstrated a significant remyelination of the $\mathrm{CC}$ within 5 days after Cuprizone withdrawal, the chronic Cuprizone model showed an impaired remyelination for at least 6 weeks (Harsan et al., 2008; Hussain et al., 2013). In the following experiment, LFB-PAS 
histochemical stainings were used to quantify the extent of de- and remyelination (dotted line) in the CC using the aforementioned a DM score (table 11, Fig. 24 A).

A

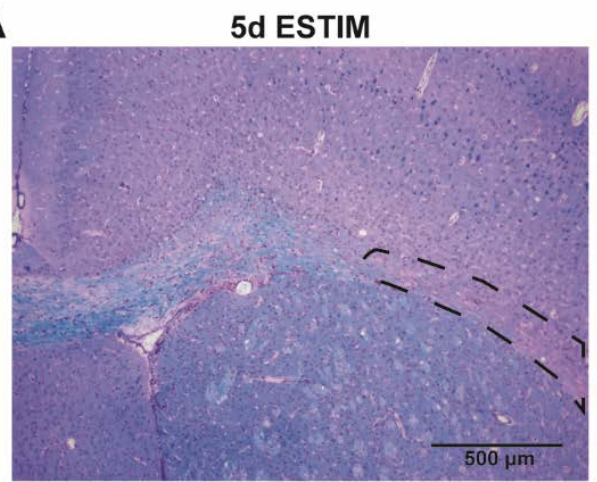

B DM score - medial corpus callosum

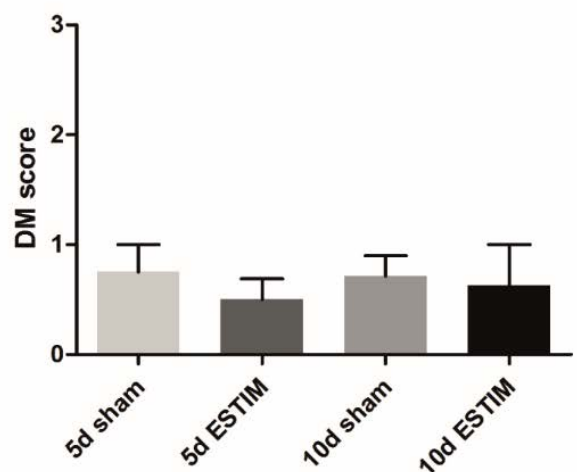

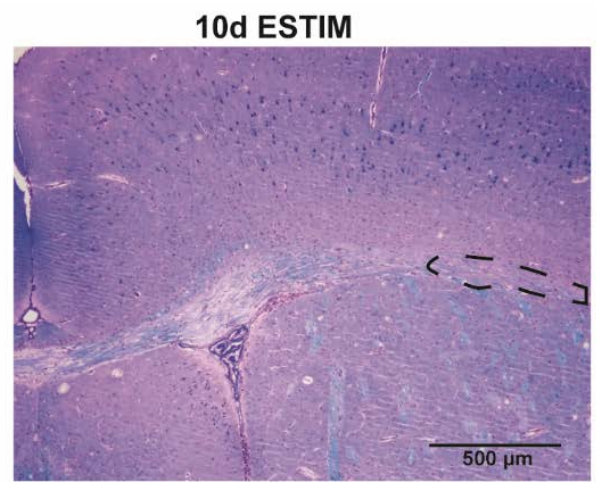

C DM score - lateral corpus callosum

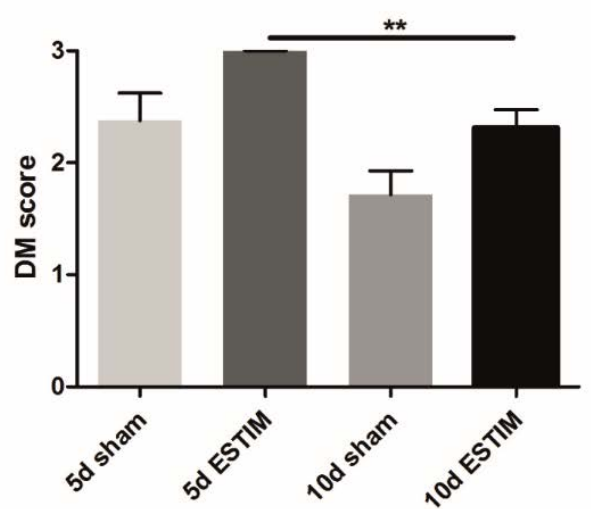

Figure 24: The extent of remyelination of ESTIM treated animals does not differ from sham treated animals

Evaluation of the extent of de- and remyelination in the CC of sham and ESTIM treated mice after $5 \mathrm{~d}$ (sham $n=8$, ESTIM $n=8$ ) and $10 \mathrm{~d}$ (sham $n=7$, ESTIM $n=7$ ) of stimulation. A Detailed view of LFB-PAS stainings of the CC after $5 d$ and $10 d$ of ESTIM. The dotted line indicates the previously demyelinated and now in part remyelinated area (scale bar: $500 \mu \mathrm{m}$ ). B Semi-quantitative analysis of the medial and C lateral area of the CC of $5 \mathrm{~d}$ sham/ESTIM $(n=8 / n=8)$ and $10 \mathrm{~d}$ sham/ESTIM $(n=7 / n=7)$ mice. Remyelination is more extensive in the medial area. ESTIM animals demonstrated significantly more remyelination of the lateral CC after 10 days of ESTIM compared to 5 days of ESTIM $\left({ }^{*} p<0.01\right.$, Mann-Whitney test). Data are shown as mean \pm SEM.

The semi-quantitative analysis revealed a more severe demyelination in the lateral part of the CC ( $5 d$ sham lateral: $2.38 \pm 0.25,5 d$ ESTIM lateral: $3.0 \pm 0,10 d$ sham lateral: $1.74 \pm 0.21$, 10d ESTIM lateral: $2.31 \pm 0.16$, Fig. $24 \mathrm{C}$ ) compared to the medial area for all experimental groups and time points ( $5 \mathrm{~d}$ sham medial: $0.75 \pm 0.25,5 \mathrm{~d}$ ESTIM medial: $0.5 \pm$ $0.19,10 \mathrm{~d}$ sham medial: $0.71 \pm 0.18,10 \mathrm{~d}$ ESTIM medial: $0.63 \pm 0.38$, Fig. $24 \mathrm{~B})$. Due to the overall low DM score in the medial $C C$, no effects between the treatment groups and time points were observed. If comparing the lateral area of sham and ESTIM treated mice, only 
the 10d ESTIM group demonstrated a significantly lower DM score compared to $5 \mathrm{~d}$ ESTIM animals $\left({ }^{*} p<0.01\right.$, Fig. $\left.24 \mathrm{C}\right)$.

\subsection{OPC and mature OLG populations in the corpus callosum remain unchanged after ESTIM}

To further evaluate the possible impact of ESTIM on remyelination, the OPC and mature OLG populations in the corpus callosum were determined. For this purpose, immunohistochemical stainings for the transcription factor Olig2 and the myelin-associated neurite growth inhibitor NogoA were quantified. Cells positive for Olig2 but negative for NogoA were considered OPC, while cells positive for NogoA were considered mature OLG (Fig. 25 A, B). Furthermore, all animals were i.p.-injected with the proliferation marker BrdU directly after Cuprizone withdrawal and every 12 hours for two consecutive days, to evaluate effects of ESTIM on cell proliferation.

A

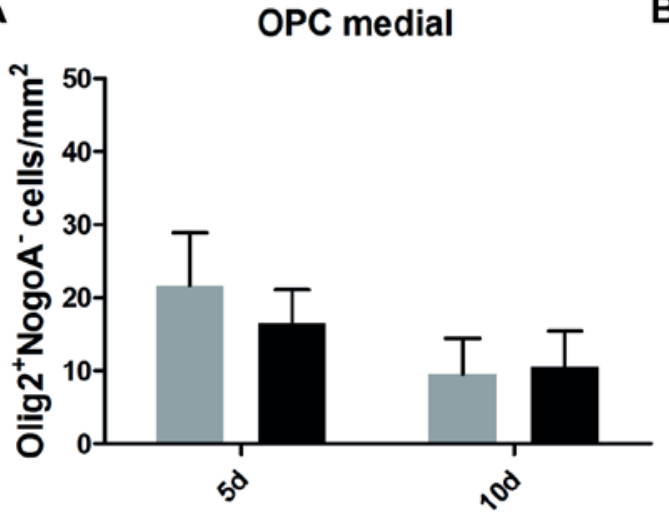

B

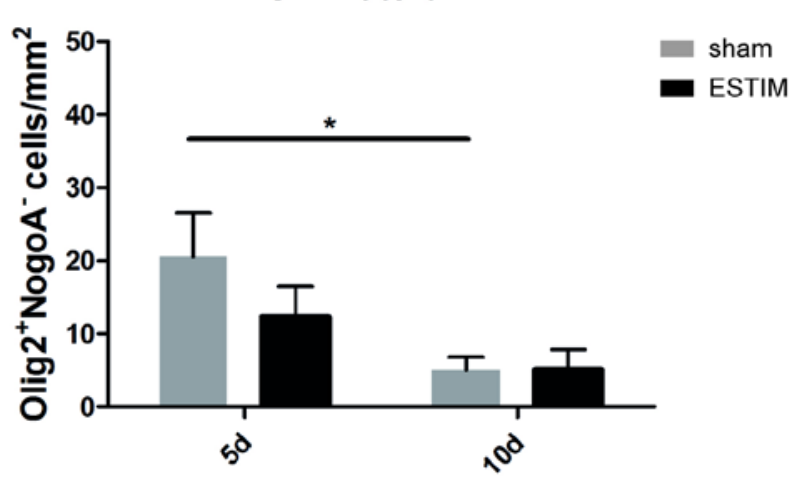

Figure 25: ESTIM did not change the density of OPC after chronic demyelination

Evaluation of the OPC population in the medial and lateral parts of the CC after either $5 \mathrm{~d}$ or $10 \mathrm{~d}$ sham or ESTIM treatment. A Olig2 ${ }^{+}$NogoA $^{-}$OPC in the medial and B lateral part of the CC of $5 \mathrm{~d}$ sham $(n=8)$ and 5d ESTIM $(n=8)$ treated mice or 10d sham $(n=7)$ and 10d ESTIM $(n=7)$ treated mice. While no significant difference in the OPC population was found between sham and ESTIM animals in neither the medial nor the lateral $C C$, sham animals demonstrated a significant decrease of OPC in the lateral CC after 10d ( ${ }^{*} p<0.05$, Two-way ANOVA followed by Sidak's multiple comparisons test). Data are shown as mean \pm SEM.

$21.69 \pm 7.2$ Olig2 $^{+} \mathrm{NogoA}^{-}$cells $/ \mathrm{mm}^{2}$ were detected in the medial area of the CC of $5 d$ sham treated animals, which did not differ from $5 \mathrm{~d}$ ESTIM animals $\left(16.5 \pm 4.55\right.$ Olig $^{+} \mathrm{NogoA}^{-}$ cells $/ \mathrm{mm}^{2}$, Fig. $25 \mathrm{~A}$ ). Similarly, no effect was found after ten days of either sham or electrical stimulation (10d sham medial: $9.55 \pm 4.88 \mathrm{Olig}^{+} \mathrm{NogoA}^{-}$cells $/ \mathrm{mm}^{2}$, $10 \mathrm{~d}$ ESTIM medial: 10.5 \pm 4.96 Olig $^{+} \mathrm{NogoA}^{-}$cells $/ \mathrm{mm}^{2}$, Fig. $25 \mathrm{~A}$ ). A different picture was observed in the lateral CC. The Two-way ANOVA analysis followed by Sidak's multiple comparisons test revealed a significant decrease of Olig2 ${ }^{+} \mathrm{NogoA}^{-}$cells in sham animals after $10 \mathrm{~d}$ compared to $5 \mathrm{~d}(5 \mathrm{~d}$ 
sham lateral: $20.58 \pm 5.99$ Olig2 $^{+} \mathrm{NogoA}^{-}$cells $/ \mathrm{mm}^{2}$, $10 \mathrm{~d}$ sham lateral: $5.06 \pm 1.77$ Olig2 ${ }^{+}$Nogo $A^{-}$cells $/ \mathrm{mm}^{2},{ }^{*} p<0.05$, Fig. 25 B). The OPC number in ESTIM mice did not differ either from sham animals or between time points (5d ESTIM lateral: $12.3 \pm 4.18$ Olig2 ${ }^{+}$Nogo $^{-}$cells $/ \mathrm{mm}^{2}, 10 \mathrm{~d}$ ESTIM lateral: $5.11 \pm 2.76$ Olig2 $^{+} \mathrm{NogoA}^{-}$cells $/ \mathrm{mm}^{2}$, Fig. 25 B).

Higher number of $\operatorname{NogoA}^{+}$mature OLG than OPC was found in the CC of sham and ESTIM treated mice. However, no effect was discovered after five and ten days of ESTIM compared to sham treated mice in the medial area and lateral area of the CC $(5 \mathrm{~d}$ sham medial: $857.5 \pm 75.19$ NogoA $^{+}$cells $/ \mathrm{mm}^{2}$, 5d ESTIM medial: $777.9 \pm 89.85$ NogoA $^{+}$ cells/mm²; 10d sham medial: $782.7 \pm 78.81 \mathrm{NogoA}^{+}$cells $/ \mathrm{mm}^{2}$, 10d ESTIM medial: $664.2 \pm$ $78.66 \mathrm{NogoA}^{+}$cells $/ \mathrm{mm}^{2}$; $5 \mathrm{~d}$ sham lateral: $567.4 \pm 93.17 \mathrm{NogoA}^{+}$cells $/ \mathrm{mm}^{2}, 5 \mathrm{~d}$ ESTIM lateral: $447.0 \pm 49.22 \mathrm{Nogo}^{+}$cells $/ \mathrm{mm}^{2}, p=0.391 ; 10 \mathrm{~d}$ sham lateral: $572.4 \pm 45.09 \mathrm{NogoA}^{+}$ cells/mm², 10d ESTIM lateral: $433.2 \pm 59.07 \mathrm{NogoA}^{+}$cells $/ \mathrm{mm}^{2}$, Fig. $26 \mathrm{~A}, \mathrm{~B}$ ).
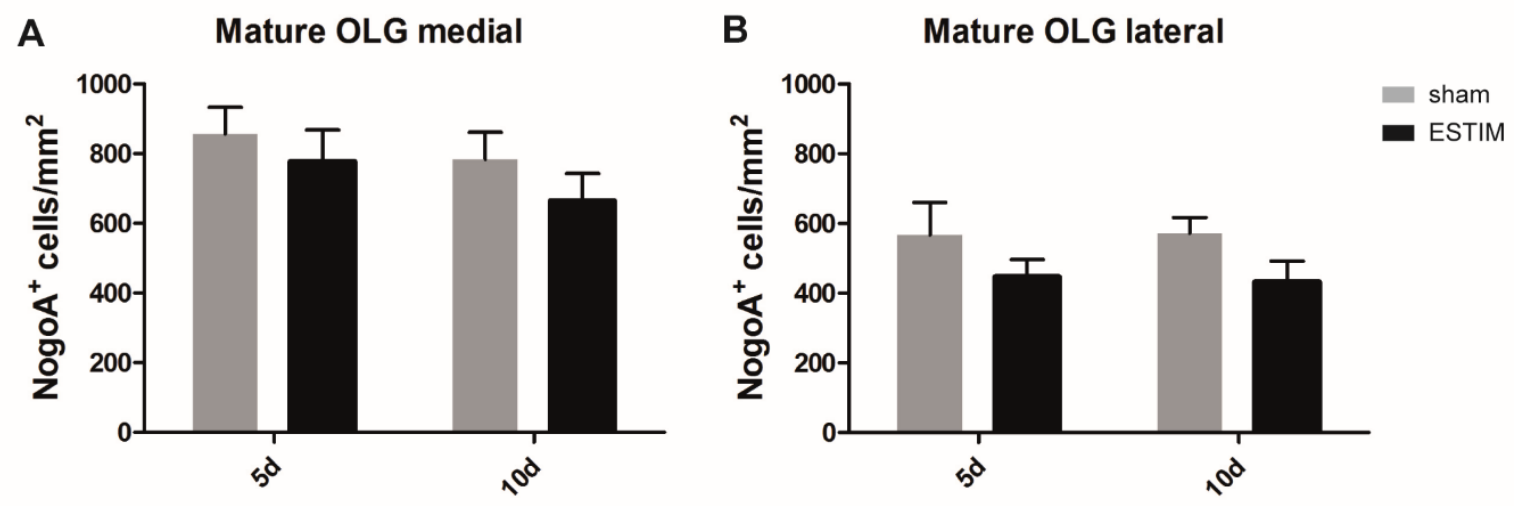

Figure 26: Numbers of $\operatorname{NogoA}^{+}$cells are not altered by ESTIM

Quantification of immunohistochemical stainings of the CC of sham ( $5 \mathrm{~d} n=8,10 \mathrm{~d} n=8)$ and ESTIM (5d $n=7,10 \mathrm{~d} n=7$ ) treated mice with antibodies against NogoA. NogoA ${ }^{+}$cells were considered mature OLG. No significant difference between the sham and ESTIM treated mice was observed after five or ten days in A the medial or B lateral area of the CC (Two-way ANOVA followed by Sidak's multiple comparisons test). Data are shown as mean \pm SEM.

Only very few Olig2 ${ }^{+}$Nogo $A^{-}$OPC were co-labeled with BrdU, indicating recent proliferation. After five days, one animal of the sham group showed $\mathrm{BrdU}^{+} \mathrm{OPC}$ in the medial as well as lateral area of the CC, while no $\mathrm{BrdU}^{+}$OPC were found in any $5 \mathrm{~d}$ ESTIM animals (5d sham medial: $0.27 \mathrm{Olig}^{+} \mathrm{BrdU}^{+} \mathrm{NogoA}^{-}$cells $/ \mathrm{mm}^{2}$, $5 \mathrm{~d}$ ESTIM medial: 0.0 Olig2 ${ }^{+} \mathrm{BrdU}^{+} \mathrm{NogoA}^{-}$cells $/ \mathrm{mm}^{2}$; $5 \mathrm{~d}$ sham lateral: 0.37 Olig2 ${ }^{+} \mathrm{BrdU}^{+} \mathrm{Nogo}^{-}$cells $/ \mathrm{mm}^{2}, 5 \mathrm{~d}$ ESTIM lateral: 0.0 Olig2 ${ }^{+} \mathrm{BrdU}^{+} \mathrm{NogoA}^{-}$cells $/ \mathrm{mm}^{2}$; Fig. $27 \mathrm{~A}, \mathrm{~B}$ ). However, after ten days only two animals of the ESTIM group and none of the sham group demonstrated $\mathrm{BrdU}^{+}$OPC in the medial area (10d sham medial: $0.0 \mathrm{Olig}^{+} \mathrm{BrdU}^{+} \mathrm{NogoA}^{-}$cells $/ \mathrm{mm}^{2}, 10 \mathrm{~d}$ ESTIM medial: $2.18 \pm 1.71 \mathrm{Olig}^{+} \mathrm{BrdU}^{+} \mathrm{NogoA}^{-}$cells $/ \mathrm{mm}^{2}$, Fig. $27 \mathrm{~A}$ ) and one animal of each group showed 
$\mathrm{BrdU}^{+} \mathrm{OPC}$ in the lateral area (10d sham lateral: 0.23 Olig2 ${ }^{+} \mathrm{BrdU}^{+} \mathrm{NogoA}^{-}$cells $/ \mathrm{mm}^{2}, 10 \mathrm{~d}$ ESTIM lateral: 0.50 Olig2 $^{+} \mathrm{BrdU}^{+} \mathrm{NogoA}^{-}$cells $/ \mathrm{mm}^{2}$, Fig. 27 B).
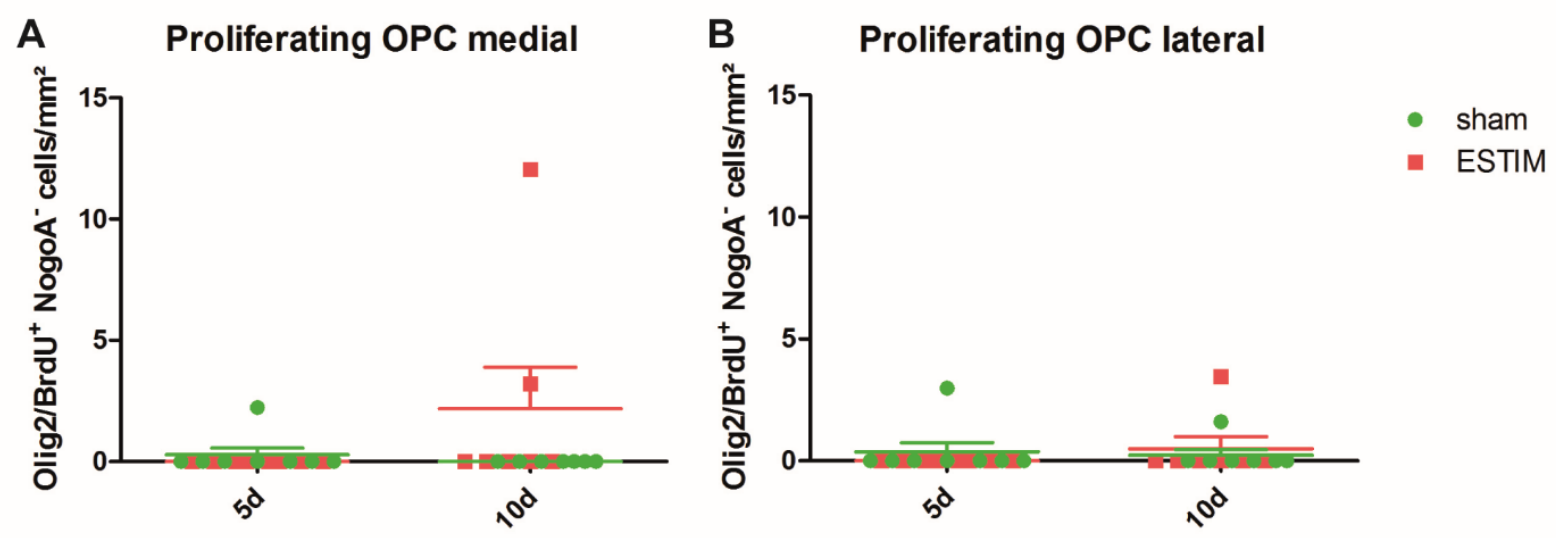

Figure 27: Overall low number of proliferating OPC after 5 and 10 days

Immunohistochemical stainings against Olig2, NogoA and BrdU were used to quantify the amount of proliferating OPC in the CC of sham ( $5 \mathrm{~d} n=8,10 \mathrm{~d} n=8)$ or ESTIM $(5 \mathrm{~d} n=7,10 \mathrm{~d} n=7)$ treated mice. Very few Olig2 ${ }^{+}$Nogo $A^{-}$cells co-labeled with $\mathrm{BrdU}$ were found in $\mathbf{A}$ the medial and $\mathbf{B}$ the lateral $\mathrm{CC}$ at both time points. Data are shown as mean \pm SEM.

In contrast to the $\mathrm{BrdU}^{+}$OPC population, a relatively high number of $\mathrm{BrdU}^{+} \mathrm{NogoA}^{+}$ cells were observed (Fig. 28 A, B). Nevertheless, no significant difference in the amount of $\mathrm{BrdU}^{+}$mature OLG was discovered in sham compared to ESTIM mice after $5 \mathrm{~d}$ and $10 \mathrm{~d}$ in the medial area (5d sham medial: $28.02 \pm 3.65 \mathrm{BrdU}^{+} \mathrm{NogoA}^{+}$cells $/ \mathrm{mm}^{2}$, 5d ESTIM medial: 18.43 $\pm 5.33 \mathrm{BrdU}^{+} \mathrm{NogoA}^{+}$cells $/ \mathrm{mm}^{2} ; 10 \mathrm{~d}$ sham medial: $32.26 \pm 9.33 \mathrm{BrdU}^{+} \mathrm{NogoA}^{+}$cells $/ \mathrm{mm}^{2}, 10 \mathrm{~d}$ ESTIM medial: $20.66 \pm 8.63 \mathrm{BrdU}^{+} \mathrm{NogoA}^{+}$cells $\left./ \mathrm{mm}^{2}\right)$ and in the lateral area of the CC (5d sham lateral: $23.31 \pm 2.21 \mathrm{BrdU}^{+} \mathrm{NogoA}^{+}$cells $/ \mathrm{mm}^{2}, 5 \mathrm{~d}$ ESTIM lateral: $12.55 \pm 4.09$ $\mathrm{BrdU}^{+} \mathrm{NogoA}^{+}$cells $/ \mathrm{mm}^{2}, 10 \mathrm{~d}$ sham lateral: $23.14 \pm 3.31 \mathrm{BrdU}^{+} \mathrm{NogoA}^{+}$cells $/ \mathrm{mm}^{2}, 10 \mathrm{~d}$ ESTIM lateral: $13.66 \pm 3.73 \mathrm{BrdU}^{+} \mathrm{NogoA}^{+}$cells $/ \mathrm{mm}^{2}$; Fig. $\left.28 \mathrm{~A}, \mathrm{~B}\right)$. 
A Proliferating mature OLG medial

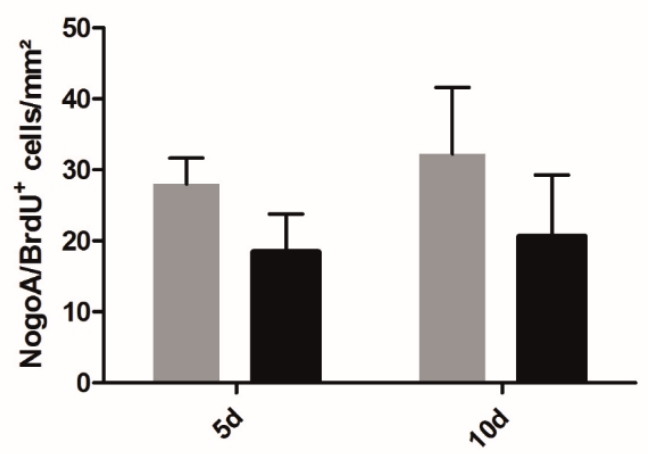

B Proliferating mature OLG lateral

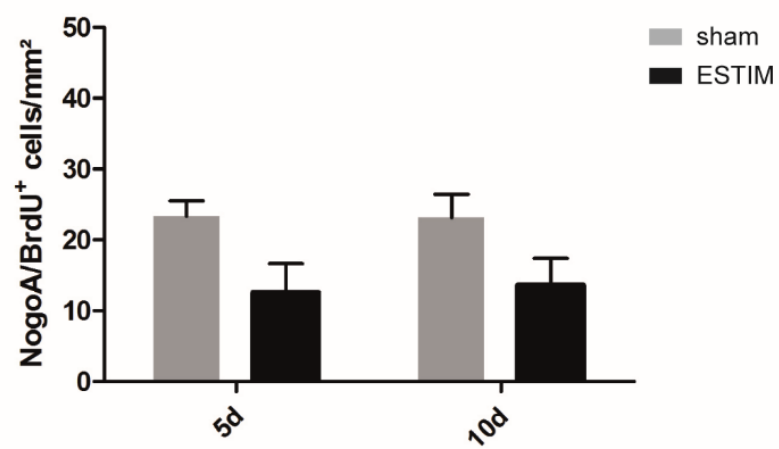

Figure 28: The amount of proliferating mature OLG is unaltered by ESTIM

Immunohistochemical stainings against NogoA and BrdU were used to quantify the amount of proliferating mature OLG in the CC of sham $(5 \mathrm{~d} n=8,10 \mathrm{~d} n=8)$ or ESTIM ( $5 \mathrm{~d} n=7,10 \mathrm{~d} n=7)$ treated mice. The density of NogoA+ mature OLG co-labeled with BrdU in $\mathbf{A}$ the medial CC and $\mathbf{B}$ the lateral CC was not influenced by ESTIM treatment. Data were analyzed by Two-way ANOVA followed by Sidak's multiple comparisons test. Data are shown as mean \pm SEM. 


\section{Part III - Results}

Transcranial electrical stimulation in healthy human subjects 


\subsection{Transcranial electrical stimulation in healthy human subjects}

The following experiments were conducted to evaluate the effect of tACS on motor behavior of healthy human subjects and are part of a clinical study (Kunz et al., 2016). tACS was applied with various eTBS patterns to healthy human subjects in a double-blinded crossover study. The amplitudes of muscle evoked potentials (MEP) were measured twice before and at 0, 10, 20, 30, 40, 50 and 60 min after the stimulation. Sham stimulation served as a control. The experiments were separated into two parts. In the first part, three different eTBS protocols (eiTBS, eimTBS, ecTBS) and one sham stimulation were delivered to fifteen healthy subjects (Fig. 8). In the second part, three altered ecTBS protocols were delivered to ten healthy subjects: $5 \mathrm{~mA}$ ecTBS with sinusoidal bursts of $5 \mathrm{~ms}$ duration, $10 \mathrm{~mA}$ ecTBS with sinusoidal bursts of $1 \mathrm{~ms}$ and $10 \mathrm{~mA}$ ecTBS with sinusoidal bursts of $5 \mathrm{~ms}$ (Fig. 8). Each stimulation was performed on a different day with at least one week in between.

\subsubsection{MEP amplitudes after eTBS did not differ from MEP after sham treatment}

Four different stimulation protocols were performed during the first part of the study: eiTBS, eimTBS, ecTBS and sham. MEP amplitudes were recorded to evaluate changes in motor behavior. Two MEP measurements were conducted before each stimulation and the mean was used to establish a baseline for each session, respectively. The MEP baseline of every subject was pooled (average baseline MEP: $1.01 \pm 0.2 \mathrm{mV}$ ) and each dataset was normalized to the respective baseline.

The normalized MEP amplitudes of eiTBS decreased significantly over time (eiTBS 0 min: $78.89 \pm 12.03 \%$, eiTBS $10 \mathrm{~min}: 73.49 \pm 11.45 \%$, eiTBS $20 \mathrm{~min}: 88.54 \pm 14.75 \%$, eiTBS 30 min: $72.17 \pm 11.04 \%$, eiTBS $40 \mathrm{~min}: 73.50 \pm 7.09 \%$, eiTBS $50 \mathrm{~min}: 82.25 \pm 10.11 \%$, eiTBS 60 min: $64.45 \pm 8.41 \%$, ${ }^{*} p<0.01$, Fig. 29 A). While eimTBS depicted only a slight decrease in MEP amplitudes (eimTBS 0 min: $104.6 \pm 9.5 \%$, eimTBS 10 min: $76.89 \pm$ 10.92\%, eimTBS $20 \mathrm{~min}: 91.86 \pm 18.16 \%$, eimTBS $30 \mathrm{~min}: 95.84 \pm 21.67 \%$, eimTBS $40 \mathrm{~min}$ : $80.56 \pm 14.22 \%$, eimTBS $50 \mathrm{~min}: 93.88 \pm 14.96 \%$, eimTBS $60 \mathrm{~min}: 95.87 \pm 15.78 \%$, $p=0.082$, Fig. $29 \mathrm{~B}$ ), an even stronger decrease of MEP size was observed for ecTBS treated subjects (ecTBS $0 \mathrm{~min}: 100.3 \pm 9.93 \%$, ecTBS $10 \mathrm{~min}: 71.63 \pm 10.42 \%$, ecTBS $20 \mathrm{~min}$ : $76.93 \pm 15.33 \%$, ecTBS $30 \mathrm{~min}: 87.62 \pm 10.25 \%$, ecTBS $40 \mathrm{~min}: 71.37 \pm 6.82 \%$, ecTBS 50 min: $68.29 \pm 8.98 \%$, ecTBS 60 min: $62.36 \pm 8.04 \%$, ${ }^{* \star *} p<0.001$, Fig. 29 C). However, a significant drop of MEP amplitudes was also registered after sham stimulation (sham 0 min: $108.8 \pm 11.52 \%$, sham 10 min: $108.4 \pm 20.22 \%$, sham 20 min: $87.19 \pm 13.27 \%$, sham 30 min: $87.05 \pm 14.9 \%$, sham 40 min: $100.7 \pm 15.01 \%$, sham 50 min: $74.16 \pm 13.01 \%$, sham 60 min: $90.34 \pm 22.22 \%,{ }^{*} p<0.05$, Fig. 29 A-C). 


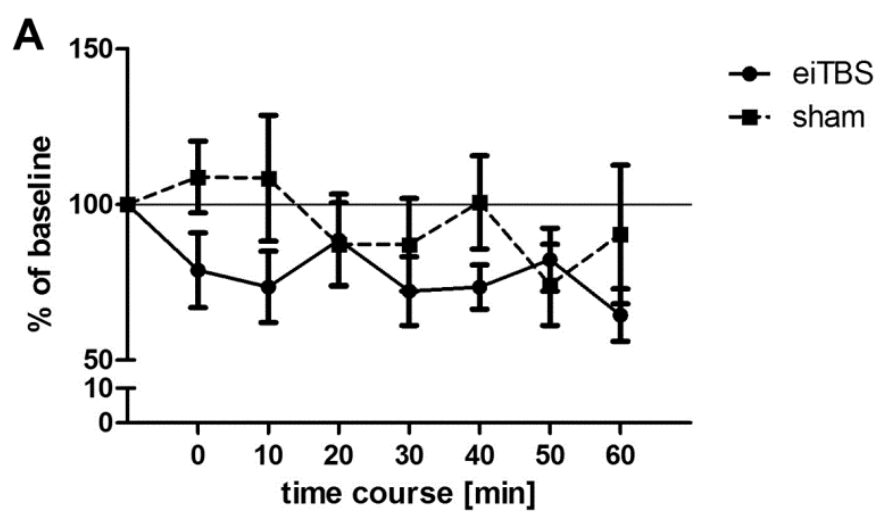

B
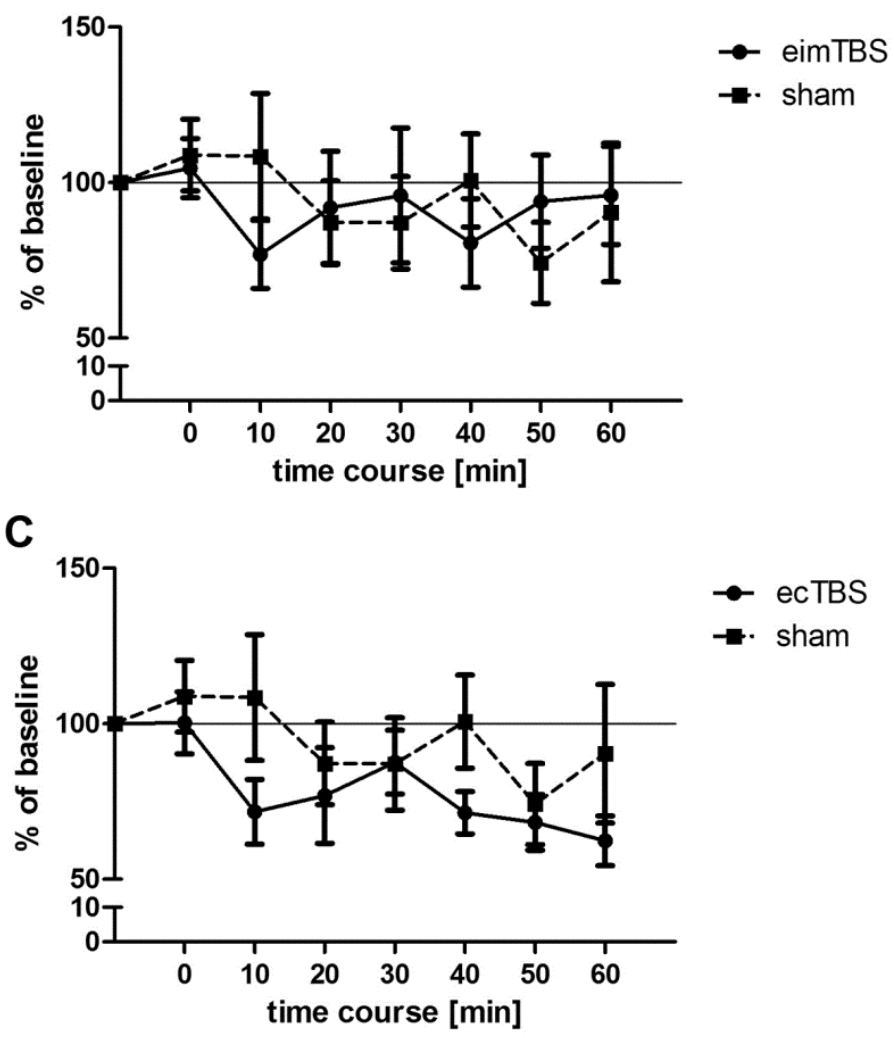

Figure 29: MEP amplitudes after eiTBS, eimTBS and ecTBS did not differ from sham

MEP responses $0,10,20,30,40,50$ and 60 min after stimulation with A eiTBS, B eimTBS and C ecTBS. Data ( $n=15)$ was normalized to the respective baseline. MEP amplitudes of eiTBS $\left({ }^{*} p<0.01\right)$, ecTBS $\left({ }^{\star \star} p<0.001\right)$ and sham $\left({ }^{\star} p<0.05\right)$ stimulated participants decreased significantly over the time course compared to baseline. Repeated measurements Two-Way ANOVA was performed to compare all eTBS protocols with each other and to compare each eTBS protocol to sham. No effect of TYPE of stimulation was observed by comparing eiTBS, eimTBS and ecTBS $(F(3,392)=0.76, p>0.05)$, nor by comparing each eTBS protocol to sham (eiTBS $F(1,196)=1.34 ; p>0.05$, eimTBS $F(1,196)=0.02$; $p>0.05$, ecTBS $F(1,196)=1.3 ; p>0.05)$. Only ecTBS demonstrated a significant effect on TIME compared to sham $\left(F(1,196)=2.853 ;{ }^{*} p<0.01\right)$. Kruskal-Wallis test followed by Dunn's multiple comparison test was performed to compare each time point to baseline. Data are shown as means \pm SEM. This Figure is part of a clinical study, published in Frontiers in Human Neuroscience (Kunz et al. 2017). 
Moreover, no significant effect on MEP amplitudes was observed by comparing all four stimulation protocols (repeated measurements Two-Way ANOVA (TYPE): $F(3,392)=0.76, p>0.05)$. Similarly, no effect of TYPE was discovered by comparing each eTBS protocol with sham (repeated measurements Two-Way ANOVA (TYPE): eiTBS $F(1,196)=1.34 ; p>0.05$, eimTBS $F(1,196)=0.02 ; p>0.05$, ecTBS $F(1,196)=1.3 ; p>0.05)$. If comparing ecTBS with sham, a significant effect of TIME was found, indicating a strong decrease of MEP amplitudes over time $\left(F(1,196)=2.853 ;{ }^{*} p<0.01\right)$. Common to eiTBS and ecTBS, but not eimTBS or sham, is a significant decrease of MEP size within the first ten minutes after stimulation compared to baseline (eiTBS/ecTBS $10 \mathrm{~min}:{ }^{*} p<0.05$ ).

\subsubsection{No significant effect on MEP amplitudes after stimulation with altered ecTBS protocols}

The second part of the study consisted of three altered ecTBS protocols. Due to the higher intensities used in this part, a sensation questionnaire was introduced, which all ten volunteers had to fill out after each session. A mild tingling sensation was reported by five participants and a medium intensity tingling sensation was reported by one participant. One further subject experienced a mildly uncomfortable sensation during the $5 \mathrm{~mA} 5 \mathrm{~ms}$ burst duration ecTBS protocol, but not during the $10 \mathrm{~mA}$ ecTBS protocols. The sensation of phosphenes was reported only by one participant during the $10 \mathrm{~mA}$ ecTBS protocol with 1 ms sinusoidal burst duration.

The average baseline amplitude was $1.01 \mathrm{mV} \pm 0.21 \mathrm{mV}$, demonstrating relatively stable baseline MEP, similarly to the first part of the study. The normalized MEP values of the $5 \mathrm{~mA}$ ecTBS protocol with $5 \mathrm{~ms}$ sinusoidal burst duration did not differ significantly from the baseline at any time point ( $5 \mathrm{~mA} 5 \mathrm{~ms} 0 \mathrm{~min}: 96.9 \pm 7.88 \%, 5 \mathrm{~mA} 5 \mathrm{~ms} 10 \mathrm{~min}: 87.58 \pm$ 12.83\%, 5 mA 5 ms 20 min: $92.98 \pm 18.41 \%, 5$ mA 5 ms 30 min: $114.7 \pm 26.29 \%, 5$ mA 5 ms 40 min: $84.62 \pm 12.84 \%$, $5 \mathrm{~mA} 5 \mathrm{~ms} 50 \mathrm{~min}: 125.7 \pm 25.89 \%$, $5 \mathrm{~mA} 5 \mathrm{~ms} 60 \mathrm{~min}: 97.97 \pm$ 18.15\%, Fig. 30). The same situation was observed for the $10 \mathrm{~mA} 1 \mathrm{~ms}$ ecTBS protocol (10 mA 1 ms 0 min: $98.02 \pm 12.86 \%, 10 \mathrm{~mA} 1 \mathrm{~ms} 10 \mathrm{~min}: 77.94 \pm 9.24 \%, 10 \mathrm{~mA} 1 \mathrm{~ms} 20 \mathrm{~min}$ : $97.83 \pm 9.8 \%, 10 \mathrm{~mA} 1 \mathrm{~ms} 30 \mathrm{~min}: 110.5 \pm 20.91 \%, 10 \mathrm{~mA} 1 \mathrm{~ms} 40 \mathrm{~min}: 99.71 \pm 13.88 \%, 10$ $\mathrm{mA} 1 \mathrm{~ms} 50 \mathrm{~min}: 110.8 \pm 13.0 \%, 10 \mathrm{~mA} 1 \mathrm{~ms} 60 \mathrm{~min}: 108.1 \pm 19.66 \%)$ and the $10 \mathrm{~mA} 5 \mathrm{~ms}$ ecTBS protocol (10 mA 5 ms 0 min: $99.87 \pm 17.42 \%$, $10 \mathrm{~mA} 5 \mathrm{~ms} 10 \mathrm{~min}: 85.93 \pm 16.02 \%$, $10 \mathrm{~mA} 5 \mathrm{~ms} 20 \mathrm{~min}: 105.7 \pm 24.64 \%, 10 \mathrm{~mA} 5 \mathrm{~ms} 30 \mathrm{~min}: 84.88 \pm 15.49 \%, 10 \mathrm{~mA} 5 \mathrm{~ms} 40$ min: $82.11 \pm 16.14 \%, 10 \mathrm{~mA} 5 \mathrm{~ms} 50 \mathrm{~min}: 77.7 \pm 17.5 \%, 10 \mathrm{~mA} 5 \mathrm{~ms} 60 \mathrm{~min}: 102.2 \pm$ 25.02\%, Fig. 30). Furthermore, no significant effect could be demonstrated by comparing all three ecTBS protocols for neither TYPE of stimulation $(F(2,189)=0.17, p>0.05)$ nor TIME $(F(7,189)=0.95, p>0.05)$. 
As depicted in Figure 31, the individual MEP responses were highly variable for every used stimulation protocol, including sham. Thus, no clear after-effect was found for any stimulation protocol.

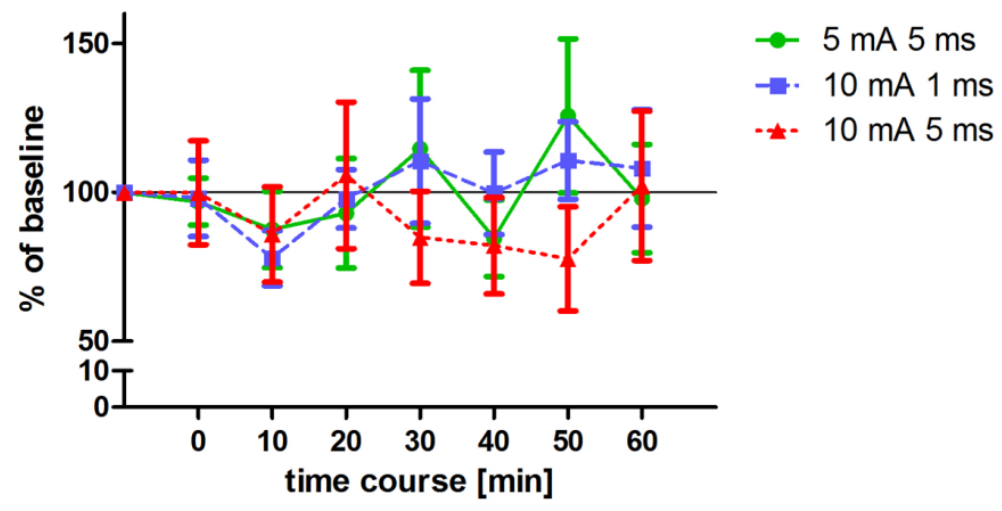

Figure 30: MEP responses remain unchanged after stimulation with altered ecTBS protocols

MEP responses $0,10,20,30,40,50$ and 60 min after stimulation with $5 \mathrm{~mA}$ ecTBS with $5 \mathrm{~ms}$ sinusoidal pulse duration, $10 \mathrm{~mA}$ ecTBS with $1 \mathrm{~ms}$ pulse duration and $10 \mathrm{~mA}$ ecTBS with $5 \mathrm{~ms}$ pulse duration. Data $(n=10)$ were normalized to the respective baseline. No significant effect on MEP size was observed for any altered ecTBS protocol compared to baseline. Furthermore, no significant effect on TYPE $(F(2,189)=0.17, p>0.05)$ of stimulation or on $\operatorname{TIME}(F(7,189)=0.95, p>0.05)$ could be observed by comparing all three protocols with repeated measures Two-Way ANOVA. Friedman's test followed by Dunn's multiple comparison test was performed to compare each time point to baseline. Data are shown as means \pm SEM. This Figure is part of a clinical study, published in Frontiers in Human Neuroscience (Kunz et al. 2017).
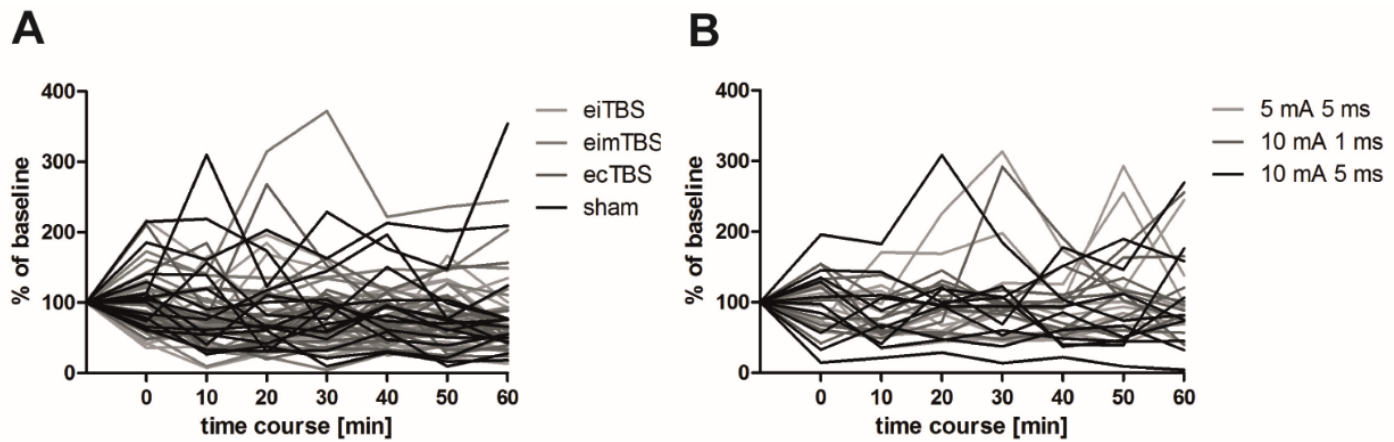

Figure 31: High interindividual variability of MEP amplitudes following eTBS and sham stimulation

Individual MEP responses of each subject participating in $\mathbf{A}$ the first study part and $\mathbf{B}$ the second study part, normalized to baseline. A high interindividual variability was registered for all stimulation protocols, including sham. This Figure is part of a clinical study, published in Frontiers in Human Neuroscience (Kunz et al. 2017). 


\section{Part I - Discussion}

Influence of autoimmune inflammation on remyelination in Cuprizone-induced demyelination 


\subsection{Influence of autoimmune inflammation on remyelination in Cuprizone- induced demyelination}

\subsubsection{Increased infiltration of the CNS by immune competent cells in Cup-EAE mice}

Multiple Sclerosis is a chronic, inflammatory disease featuring demyelinated lesions in the gray and white matter (Noseworthy et al., 2000; Stadelmann et al., 2011). Infiltrating $\mathrm{CD}^{+}$and $\mathrm{CD}^{+} \mathrm{T}$ cells as well as $\mathrm{B}$ cells are key players in mediating the inflammatory cascade during MS, leading to primary demyelination and neuroaxonal degeneration (Noseworthy et al., 2000; Popescu and Lucchinetti, 2012; Weiner, 2004). However, it was recently discussed whether neurodegenerative events such as oligodendrocyte death, microglia activation as well as axonal damage, and not the adaptive immune system, are the initial factors in driving MS lesion formation (Barnett and Prineas, 2004). Nevertheless, the most common MS animal models were only able to mirror certain aspects of this disease, like infiltration of immune competent cells followed by demyelinated lesions, which can mainly be found in the spinal cord (EAE; Mendel et al., 1995) or toxin induced CNS demyelination with sparse involvement of inflammatory infiltrates (Cuprizone; injection of ethidiumbromide/lysolecithin; Jeffery and Blakemore, 1995; Matsushima and Morell, 2001). Interestingly, the immunization of Cuprizone fed animals with $\mathrm{MOG}_{35-55}$ peptide led to inflammatory, demyelinated lesions in the brain, as was demonstrated by Boretius and colleagues in 2012 and subsequently by Scheld et al. in 2016 (Boretius et al., 2012; Scheld et al., 2016).

In line with this, we showed that the combination of Cuprizone administration with EAE induction led to a strong infiltration of the CNS with T cells, NK cells, B cells, granulocytes and inflammatory monocytes accompanied by demyelination of the corpus callosum $(3.1 .1,3.1 .2)$. It is widely accepted that the adaptive immune system plays a major role during MS, and the upregulation of $T$ and $B$ cells is central to most EAE models (Mendel et al., 1995; Weiner, 2004). B cells, for instance, play a multifactorial role in auto-immune diseases: they can secrete antibodies in the form of plasma cells and serve as antigen presenting cells, thus exerting immune modulatory purposes (Mizoguchi and Bhan, 2006). According to the cytokine expression pattern, B cells can be separated into two subsets: "effector" B cells, which express pro-inflammatory cytokines like TNFa, IFNy, IL-6 or IL-12 and "regulatory" B cells, which express anti-inflammatory cytokines like IL-10 or transforming growth factor- $\beta$ (TGF $\beta-1)($ Lund, 2008; Mizoguchi and Bhan, 2006; Wolf et al., 1996). Therefore, raised levels of $B$ cells in autoimmune diseases can exert different effects on the immune response, depending on the $B$ cell subset.

Besides the adaptive immune system, growing evidence for an involvement of the innate immune system in MS can be found (e.g. Weiner, 2008). It was shown that agents of 
the innate immune system with immunomodulatory properties like NK cells, granulocytes and inflammatory monocytes could potentially influence the disease course of MS as well as EAE (Gandhi et al., 2010). NK cells, for instance, can be divided into two subsets: CD56 ${ }^{\text {high }}$ and CD56 ${ }^{\text {low }}$. The CD56 ${ }^{\text {low }}$ subsets seems to exert mainly cytotoxic functions, while CD56 ${ }^{\text {high }}$ secretes anti-inflammatory cytokines and therefore might perform a regulatory function (Cooper et al., 2001). Granulocytes, on the other hand, act as phagocytes in response to inflammation. Neutrophil granulocytes are activated by $T_{H} 17$ cells expressing molecules like granulocyte-colony stimulating factor or the ELR ${ }^{+}$CXC chemokine CXCL1 and were found to infiltrate the CNS during EAE (Pelletier et al., 2010; Rumble et al., 2015). Inflammatory monocytes, which subsequently can differentiate into antigen presenting cells like dendritic cells or macrophages, represent a further factor of the innate immune system that may impact the disease course of MS or EAE by secretion of pro-inflammatory cytokines like IFNY or TNFa or by phagocytosis of myelin (Huang et al., 1999). Furthermore, the increase of inflammatory monocytes in the brain may indicate that at least a part of the antigen presenting cells mediating the immune response in the Cup-EAE model might be represented by macrophages. A similar role is played by activated microglia, which also serve as antigen presenting cells, clear myelin debris by phagocytosis and mediate the immune response by expression of pro-inflammatory cytokines and enzymes like IL-1 $\beta$ and inducible nitric oxide synthase (iNOS)(Gray et al., 2008; Mosley and Cuzner, 1996). As it was shown in the present study, the microglia population in the CNS of Cuprizone animals was not further increased after immunization with $M O_{35-55}$ peptide (3.1.2). This was expected, since in the Cuprizone model, microglia are responsible for the clearance of myelin debris and are therefore fully activated (Hiremath et al., 1998). Hence, it can be assumed that the induction of EAE and subsequent breach of the BBB would not increase the activation of microglia any further.

The increased infiltration of not only $T$ and $B$ cell populations in the Cup-EAE model but also of important players of the innate immune system demonstrates a successful inflammatory signaling cascade and the infiltration of immune competent cells, which correlates with reports in MS patients (Compston and Coles, 2008).

\subsubsection{Damaging and neuroprotective effects of autoimmune inflammation}

As demonstrated in the doctoral thesis of A. Escher, the infiltration of myelin-specific $T$ cells into the CNS of cuprizone animals by immunization with MOG $_{35-55}$ peptide led to increased expression of the pro-inflammatory cytokine IFNy as well as raised levels of the pro-inflammatory molecule iNOS (Escher, 2008). IFNy is expressed by inflammatory cells like autoreactive $\mathrm{CD}^{+} \mathrm{T}_{\mathrm{H}} 1 \mathrm{~T}$ cells, NK cells or macrophages and activates antigen presenting cells in the CNS, represented by microglia, macrophages and astrocytes (Cao et al., 2015; 
Martins et al., 2011). Activated macrophages and microglia, in turn, produce reactive oxygen species by expressing iNOS, which are highly toxic for most pathogenic microorganisms (Dale et al., 2008; Nathan et al., 1983). Increased levels of reactive oxygen species however, can lead to lipid peroxidation and axonal damage (Nathan and Ding, 2010; di Penta et al., 2013). Indeed, an increase in $\mathrm{APP}^{+}$axons in Cuprizone mice immunized with $\mathrm{MOG}_{35-55}$ peptide was detected in the lateral $\mathrm{CC}$, indicating impaired axonal transport and therefore increased axonal damage compared to Cuprizone only treated mice (3.1.5).

On the other hand, it was speculated that an inflammatory milieu might be beneficial in terms of neuroprotection and remyelination (Hohlfeld, 2007). It was discovered that, upon activation, immune cells can secrete neurotrophic factors like BDNF, neurotrophin-3, LIF or glial cell line-derived neurotrophic factors like neurturin (Kerschensteiner et al., 1999, 2003; Moalem et al., 2000). BDNF, for instance, is associated with enhanced neuron and immune cell survival (Kerschensteiner et al., 1999) and can be found in inflammatory infiltrates in the brains of MS patients (Stadelmann et al., 2002). Neurotrophin-3 and LIF were shown to support oligodendrocytes, neurotrophin-3 by promoting the differentiation from OPC into mature oligodendrocytes (Cohen et al., 1996) and LIF by increasing oligodendrocyte survival in EAE (Butzkueven et al., 2006). Furthermore, neurturin might represent a signaling pathway by which immune cells are able to control the secretion of TNFa (Vargas-Leal et al., 2005). Taken together, it appears that inflammation might not only exert detrimental but also neuroprotective effects. As mentioned at the beginning of this thesis, the early stages of MS demonstrate a widespread inflammation but also the ability to remyelinate demyelinated lesions, while during the progressive stage of MS, both the inflammatory milieu in the CNS as well as the remyelination capacity of the CNS decreases (Goldschmidt et al., 2009; Kutzelnigg et al., 2005; Serafini et al., 2004).

\subsubsection{Influence of adaptive inflammation on oligodendrocyte populations and remyelination}

In contrast to the discussed neuroprotective effects, the infiltration of peripheral immune cells in Cuprizone treated animals by EAE induction did not lead to increased numbers of OPC or myelin producing oligodendrocytes, or to increased remyelination, as indicated by Olig2/NogoA immunohistochemical stainings (3.1.3), PLP mRNA in situ hybridization as well as LFB-PAS histochemical stainings (3.1.4), respectively. Furthermore, electron microscopic analysis of the corpus callosum of Cuprizone and Cuprizone-EAE animals revealed no significant difference in the density of myelinated axons between the groups, neither after 4.5 weeks of Cuprizone administration nor after five days on a Cuprizone-free diet (3.1.6). Regarding the quantification of oligodendrocyte populations by Olig2/NogoA immunohistochemical stainings, it seemed that the remyelination process might 
even be slightly impaired after immunization with MOG-peptide. Although no significant effect on the OPC number (Olig ${ }^{+} \mathrm{NogoA}^{-}$cells) could be found by directly comparing the two experimental groups, the Cuprizone group depicted significantly less OPC in the lateral CC after five days on cuprizone-free diet. This could hint at an impairment or delay of OPC differentiation by adaptive inflammatory infiltrates. In line with this, Lin and colleagues demonstrated in 2006 that IFNy altered the time course of OPC recruitment into demyelinated lesions in Cuprizone treated mice as well as reduced the number of oligodendrocytes and impaired the remyelination in EAE animals (Lin et al., 2006). In addition, a recent study showed that EAE induced inflammation results in a downregulation of the OPC differentiating agent thyroid hormone and subsequent impairment of OPC differentiation (Fernández et al., 2016). However, Cup-EAE animals showed only a trend toward a decreased number of mature oligodendrocytes (NogoA ${ }^{+}$cells) in the medial corpus callosum compared to Cup animals but no significant effect. Therefore, according to Olig2/NogoA immunohistochemical stainings, a slight delay of OPC differentiation due to the inflammatory milieu in Cup-EAE mice remains possible.

On the other hand, the present study did not discover any differences in the amount of PLP mRNA ${ }^{+}$oligodendrocytes after five days on a Cuprizone-free diet between the two experimental groups. One reason for this discrepancy could be the staining technique. Kuhlmann and colleagues demonstrated in 2007 that in EAE lesions, fewer PLP mRNA ${ }^{+}$ oligodendrocytes were labeled compared to Nogo $^{+}$cells (Kuhlmann et al., 2007). It was suggested that PLP mRNA expression might be more sensitive to autoimmune-mediated demyelination than the expression of proteins like NogoA (Kuhlmann et al., 2007; Morell et al., 1998). In this case, the PLP mRNA in situ hybridization might be the more reliable technique. Furthermore, the data obtained from semi-quantitative demyelination analysis of LFB-PAS stainings as well as the analysis of the density of myelinated axons by electron microscopy supports the notion that a myelin-peptide-specific autoimmune response does not influence the degree of remyelination in our model of CNS demyelination. In fact, demyelination itself seems to be a sufficient trigger for remyelination, since both experimental groups displayed a similar increase in myelinated axons after five days on Cuprizone-free chow.

We demonstrated here that the experimental Cup-EAE model is able to combine major hallmarks of MS in one model: demyelinated lesions in the brain, the infiltration of peripheral immune cells and axonal damage. Furthermore, we were able to verify that the infiltration of adaptive immune cells does neither substantially impair nor ameliorate the remyelination process of the demyelinated lesion. Finally, the Cup-EAE model proved to be a legitimate tool to study remyelination in the presence of peripherally activated CNSautoreactive T cells. 


\section{Part II - Discussion}

Influence of transcranial alternating current stimulation on spontaneous remyelination in in vitro and in vivo models 


\subsection{Influence of transcranial alternating current stimulation on spontaneous remyelination in in vitro and in vivo models}

Electrical stimulation might serve as a promising tool to improve OPC differentiation, remyelination and nerve regeneration, as indicated by recent in vitro and in vivo studies (Gary et al., 2012; Huang et al., 2013; Ishibashi et al., 2006; Sherafat et al., 2012). However, most in vivo studies were conducted on exposed nerves of the PNS (Al-Majed et al., 2000; Huang et al., 2013; McLean et al., 2014; Wan et al., 2010) and only very few studies engaged in, mostly invasive, electrical stimulation of the CNS (Jahanshahi et al., 2013; Morimoto et al., 2011; Sherafat et al., 2012). The aim of the second part of this thesis was to apply electrical currents in a safe and non-invasive way to two different animal models of CNS demyelination and to provoke increased OPC proliferation and differentiation as well as improved remyelination. For this purpose, the safety and efficiency of the stimulation protocol obtained from published in vitro studies (Gary et al., 2012; Ishibashi et al., 2006) was initially tested in oligodendrocyte and mixed cortical cultures.

\subsubsection{Electrical stimulation in oligodendrocyte and mixed cortical cultures}

\subsubsection{Safe application of $10 \mathrm{~mA}$ alternating currents}

Gary and colleagues demonstrated in 2012 that the electrical stimulation protocol of $10 \mathrm{~mA}, 10 \mathrm{~Hz}$ biphasic stimulation applied to mixed cortical cultures (dorsal root ganglion [DRG] neurons, oligodendrocytes, astrocytes) for one hour daily over seven days led to increased numbers of $\mathrm{MBP}^{+}$oligodendrocytes (Gary et al., 2012). Furthermore, the group showed that this effect was reversed by addition of the voltage-dependent sodium channel inhibitor tetrodotoxin (TTX), therefore indicating a dependency on the presence of neurons. The initial experiments in the second part of this thesis were performed on oligodendrocyte cultures to verify the safe and harmless application of $10 \mathrm{~mA}, 10 \mathrm{~Hz}$ biphasic electrical currents. Indeed, the number of oligodendrocytes in ESTIM cultures did not differ from untreated controls, as indicated by $\mathrm{O} 4$ immunocytochemical stainings (3.2.1.1). Both treatment groups depicted an increase in O4 MBP double positive cells over the time course of four days, beginning at day two. The electrical stimulation of oligodendrocytes did not increase the expression of MBP in the absence of neurons, which was expected due to the findings of Gary et al. in 2012.

\subsubsection{Electrical stimulation failed to increase myelination in vitro}

Contrary to the data demonstrated by Gary and colleagues (Gary et al., 2012), electrical stimulation of mixed cortical cultures consisting of CNS neurons, astrocytes and oligodendrocytes did not lead to improved myelination, as indicated by the amount of 
myelinated axons analyzed in 3.2.1.2. Although the cytotoxicity was not increased after electrical stimulation (3.2.1.2), the cell viability over the time course of four days after merging neuronal and oligodendrocyte cultures was relatively low for both treatment groups (50-70\% cytotoxicity). This might have influenced the overall low outcome of maximal $20 \%$ myelinated axons of the total axonal fiber count in both conditions (3.2.1.2). It could be assumed that an improvement of the mixed cortical cell culture in terms of cell viability and overall degree of myelination might reveal ESTIM mediated effects, which were missed otherwise.

As mentioned before, the beneficial effect of electrical stimulation on myelination is dependent on the presence of neurons and several groups reported that neuronal activity leads to the release of cytokines and neurotransmitter such as ATP and cyclic adenosine monophosphate (cAMP), which ultimately increases myelination (Ishibashi et al., 2006; Malone et al., 2013; Stevens et al., 2002). Malone and colleagues demonstrated that in response to electrical stimulation, cultivated DRG neurons released CAMP and subsequently, an increase of myelination by co-cultivated oligodendrocytes was observed (Malone et al., 2013). The addition of extracellular cAMP to the cell culture media mimicked the beneficial effect of electrical stimulation on myelination and therefore Malone et al. concluded that the increased myelination might be regulated by neuronal activity via a cAMP pathway (Malone et al., 2013). Hence, a failure in evoking neuronal activity by electrical stimulation could result in negative results as observed in the present work. Although, we used the same stimulation protocol with a similar application method as demonstrated by Gary et al. in 2012. However, instead of rat DRG neurons, we performed the experiments with rat CNS neurons. We based this decision on the fact that the subsequent in vivo experiments of CNS demyelination are more closely resembled by CNS neurons. Yet, a recent study discovered that cultivated CNS neurons require a maturation period of approximately 25 days before achieving full firing functionality, while DRG neurons reach functional maturity already after five days in vitro (Bourke et al., 2014). Since the CNS neurons in our experiments spent a maximum of 18 days in culture (14 days pre-ESTIM + 4 days ESTIM/NOSTIM), the impaired firing ability of pre-mature CNS neurons might be another cause for the inability of the electrical stimulation protocol to improve myelination in vitro.

\subsubsection{Electrical stimulation in rodent models of demyelination}

\subsubsection{Induction of focal demyelinated lesions after intracortical injection of anti-MOG antibody and complement}

Since the intensity of the applied electrical fields would decrease in deeper brain structures, we targeted the cortex to induce demyelinated lesions, an area where the electrical stimulation would be most effective. The stereotactical injection of the anti-MOG 
antibody Z2 in combination with mouse complement in Lewis rats led to cortical as well as extensive subpial demyelinated lesions with only minimal damage to axonal fibers indicated by MBP immunohistochemistry and Bielschowsky silver impregnation (3.2.2.1.1). Such extensive subpial lesions are frequently found in MS patients and are, in contrast to white matter lesions, more specific to MS (Moll et al., 2008).

$\mathrm{Z2}$ is an IgG2 antibody specific for the myelin protein MOG. It can be assumed that it attaches to myelin sheaths and forms antigen-antibody complexes, which can be recognized directly by microglia or by the target recognition molecule C1q. C1q is part of the classical complement pathway and amplifies the immune reaction by $\mathrm{C} 3 \mathrm{~b}$ attachment to the target (Pangburn et al., 2008). C3b in turn initiates the cell destruction by assembling C5b-9 pores in the cell membrane of the target cell (membrane attack complex), which allow water influx and metabolite efflux as well as marks the target for phagocytosis by microglia. Furthermore, C3b tags antigens so they can be processed by antigen presenting cells, thus facilitating the immune response (Pangburn et al., 2008). Inflammatory mediators can be released after activating the complement cascade, which might result in an opening of the BBB and subsequent focal infiltration of macrophages as well as few $T$ and $B$ cells. Therefore, an involvement of further immune competent cells besides microglia cannot be excluded. However, it was shown that inflammation in cortical areas dissipates quickly (Merkler et al., 2006), thus, even in the case of infiltration by inflammatory cells, a persisting inflammation in this model of focal demyelination is unlikely. Besides the complement system, antibodydependent cellular cytotoxicity (ADCC) is as likely to play an important role in mediating demyelination during a pathogenic antibody response. Lagumersindez-Denis and colleagues used a similar model of cortical demyelinated lesions utilizing sub-threshold immunization with $\mathrm{MOG}_{35-55}$ peptide and stereotactical injections of the pro-inflammatory cytokines TNFa and IFNy (Lagumersindez-Denis et al., 2017). They demonstrated the crucial role of antibody-opsonization and NK cell mediated ADCC during demyelinated lesion formation in a myelin-specific antibody response (Lagumersindez-Denis et al., 2017).

The size of the demyelinated area in our model decreased gradually over the time course of 21 days in ESTIM, NOSTIM and sham animals, with first signs of remyelination observed after seven days post injection (3.2.2.1.1, 3.2.2.1.2). Similar results were observed in the focal EAE model of cortical demyelination by Merkler and colleagues, depicting a peak of demyelination around three days post injection with a reduction of the demyelinated area after seven days (Merkler et al., 2006). In the present work, the NOSTIM group depicted an almost complete remyelination after 14 and 21 days, whereas the size of the demyelinated area of both sham and ESTIM treated animals was still slightly larger at those time points, although not significantly. This indicates that the application of the electrodes on the cranium and therefore surgical opening of the scalp might induce an irritation of the area of 
stimulation, which possibly influenced the lesion regeneration. Unfortunately, a side-effect of the stereotactical injection is a suture on the scalp of the rats, which renders a completely non-invasive stimulation technique without re-opening of the scalp impossible.

The demyelinated area of the ESTIM treated animals at $d 5$ and $d 7$ was increased compared to NOSTIM animals, while sham treated animals showed no significant change compared to NOSTIM or ESTIM rats at those time points. Two possible explanations for this effect could be that either the electrical stimulation impaired remyelination processes at an early time point or the clearance of myelin debris by microglia was improved, which would also result in less $\mathrm{MBP}^{+}$signals. As OPC are responsible for remyelination (Keirstead and Blakemore, 1997; Prayoonwiwat and Rodriguez, 1993; Targett et al., 1996), any impact on them by electrical stimulation would also influence remyelination. In order to remyelinate, OPC have to differentiate into myelin forming, mature oligodendrocytes. As demonstrated by Kirson and colleagues in 2007, high frequency alternating electrical currents (>100 kHz) are able to interfere with the formation of spindle tubulin during mitosis (TTF, Kirson et al., 2007). Although the frequency of $10 \mathrm{~Hz}$ used in the present experiments was much lower than the ones used in TTF, it remains possible that our stimulation protocols impaired OPC proliferation and/or differentiation.

As mentioned above, another possible explanation for a larger area of MBP loss might be an improved clearance of myelin debris. Indeed, a recent study indicated an increase of $\mathrm{Iba1}^{+}$activated microglia in the rat cortex following transcranial electrical stimulation (Pikhovych et al., 2016). Moreover, a study performed on focal demyelination in a rat peripheral nerve demonstrated enhanced myelin clearance by ED- $1^{+}$macrophages after electrical stimulation with $20 \mathrm{~Hz}$ (McLean et al., 2014). Taken together, electrical stimulation might be able to improve microglia activation in the rat cortex as well as phagocytic myelin uptake. Further analysis of microglia/macrophage activation in the cortical lesions is required and already planned to verify the presence of macrophages and possibly increased microglia activation after electrical stimulation.

\subsubsection{Robust remyelination in sham and ESTIM animals}

Immunohistochemical stainings for Olig2 and p25 were performed to assess possible effects of electrical stimulation on oligodendrocyte populations inside the demyelinated area, in the perilesional area, the remyelinated area as well as the NAGM (3.2.2.1.3). Both treatment groups displayed a slight drop in OPC numbers (Olig2 $\left.{ }^{+} \mathrm{p} 25^{-}\right)$in the remyelinated area, the NAGM and the demyelinated area, while no change for any group was observed in the perilesional area. The decrease in the amount of OPC was expected and presumably demonstrated the steady differentiation of OPC into mature oligodendrocytes. Despite the fact that previous studies reported an increased recruitment of OPC from the SVZ after 
electrical stimulation in CNS models of demyelination and ischemic stroke (Jahanshahi et al., 2013; Sherafat et al., 2012), the OPC populations of ESTIM treated animals remained unchanged compared to sham treated animals. While the number of mature oligodendrocytes $\left(\mathrm{p} 25^{+}\right)$remained stable over the time course in the RM area as well as the NAGM and no difference was detected between the two treatment groups in any counting area, the $\mathrm{p}^{2} 5^{+}$cell count decreased over time in the DM lesion and increased in the perilesional area for sham as well as ESTIM rats. Mature oligodendrocytes are required for a successful remyelination and an increase of $\mathrm{p}^{2} 5^{+}$cells in the lesion periphery is therefore not surprising. The slow decline in the number of mature oligodendrocytes inside the demyelinated lesion, however, was unexpected, especially in context with the observed remyelination. It can be discussed that the expression of mature oligodendrocyte proteins like NogoA and p25 may be up-regulated in early demyelinated lesions, which might have caused an underestimation of the true mature oligodendrocyte count at the later time points. Furthermore, no increase in proliferating Olig2 ${ }^{+}$oligodendrocytes was observed by the analysis of immunohistochemical stainings for the proliferation marker BrdU (3.2.2.1.4) in the subpial and cortical area after electrical stimulation. In fact, the number of BrdU/Olig2 double positive cells remained relatively stable over the observed time course in every counting area. Since proliferating OPC are required for the observed successful remyelination, this could indicate that the proliferation happens at a very early time point between $\mathrm{d} 1$ and $\mathrm{d} 3$.

According to several studies, axonal activity can influence OPC proliferation/differentiation by release of cytokines and neurotransmitters such as glutamate or ATP (Bergles et al., 2000; Ishibashi et al., 2006; Stevens et al., 2002). Both glutamate and ATP were shown to affect $\mathrm{Ca}^{2+}$ responses in OPC (Bergles et al., 2000; Wake et al., 2011). $\mathrm{Ca}^{2+}$ is an important signaling molecule and involved in many processes like proliferation, migration or cell-cell communication (Berridge et al., 2000). However, it is still discussed, whether glutamatergic synapses between OPC and neurons inhibit or facilitate OPC proliferation (Maldonado and Angulo, 2015). This is due to controversial data, indicating for instance, an increase in proliferating OPC in the developing rat optic nerve following electrical stimulation (Barres and Raff, 1993) or on the other hand a decrease of $\mathrm{NG}^{+}$OPC proliferation in response to glutamatergic synaptic activity in acute cortical slices (Mangin et al., 2012).

By applying electrical currents during the initial phase of the lesion formation, we tried to influence OPC proliferation and to increase oligodendrocyte differentiation. Despite the controversial data mentioned before, we did not observe an increase or a decrease of proliferating oligodendrocytes or any changes in the number of mature oligodendrocytes following electrical stimulation. A reason for this could be the low stimulation intensity, which might be insufficient to induce increased axonal firing. Due to the fact that this setup of 
transcranial alternating current stimulation in a rat model of cortical demyelination is the first of its kind, no safety protocols exist yet. We chose a relatively low stimulation intensity to not induce heat-related lesions, as they can be found with higher stimulation intensities during transcranial direct current stimulation in rats (Liebetanz et al., 2009). We were aware that the stimulation protocol might not be able to induce action potentials in neurons on its own. However, we hypothesized that it might very well be able to influence neurons, which just received a subthreshold synaptic input that did not depolarize the neuron enough to induce an action potential. Depolarizing those neurons beyond the $-50 \mathrm{mV}$ threshold with electrical stimulation might therefore result in action potentials. Nevertheless, the lack of effects on cell proliferation and differentiation might indicate the need for stimulation protocols with higher intensities. Future experiments should therefore establish safety protocols, to evaluate the maximum stimulation intensity and duration possible that one can apply before provoking heat-induced lesions.

As indicated by the assessment of the demyelinated area of NOSTIM animals, the remyelination in this model of focal demyelination is the very robust and rapid. The window of opportunity to influence the remyelination in a model that remyelinates almost completely within 14-21 days is small. Therefore, we performed similar electrical stimulation experiments in a mouse model of chronic, Cuprizone-induced demyelination.

\subsubsection{Heterogeneous remyelination of the corpus callosum after 13 weeks of Cuprizone administration}

It was reported that the continuous administration of Cuprizone over a time course of 12 weeks led to delayed remyelination in C57BI/6 mice of at least 6 weeks (Harsan et al., 2008; Hussain et al., 2013; Matsushima and Morell, 2001). After 6 weeks of Cuprizone exposure, signs of remyelination can generally be seen, even in the presence of continuous Cuprizone exposure (Matsushima and Morell, 2001). However, if Cuprizone administration continues for another 6 weeks, the newly formed myelin and mature oligodendrocytes along with resident OPC are depleted (Mason et al., 2004). Therefore, the exhaustion of progenitor cells in the corpus callosum and the vicinity would delay further attempts of remyelination.

In line with this, we could demonstrate that the lateral part of the corpus callosum of C57BI/6 mice indeed showed a high demyelination score after five as well as after ten days of cuprizone free diet (3.2.2.2.1). The medial part of the corpus callosum on the other hand, depicted extended areas of thin $\mathrm{LFB}^{+}$signals, likely indicating newly formed myelin as well as strong $\mathrm{LFB}^{+}$signals, indicating remaining myelin, which presumably was not affected by the lengthy Cuprizone treatment. Regional differences in demyelination after Cuprizone administration were reported before and were not only observed between different white matter tracts of the CNS (Schmidt et al., 2013) but also between the lateral and medial 
corpus callosum (Tagge et al., 2016). Thus, the medial corpus callosum seemed to be less affected by the Cuprizone treatment than the lateral corpus callosum. This could result in more OPC available for remyelination or even less demyelination in the first place. Although the analysis of immunohistochemical stainings for Olig2 and NogoA revealed no differences in OPC numbers (Olig2 ${ }^{+} \mathrm{NogoA}^{-}$) between the medial and lateral corpus callosum (3.2.2.2.2.), the population of mature oligodendrocytes $\left(\operatorname{Nogo}^{+}\right)$was significantly higher in the medial area compared to the lateral area for each time point and treatment condition. Therefore, an increased differentiation of OPC into mature oligodendrocytes would result in a relatively low OPC population, in fact as low as the number of OPC of the more severely affected lateral corpus callosum. Taken together, the reduced demyelination score of the medial corpus callosum could be explained by a less severe effect of Cuprizone in this area, resulting in increased differentiation of OPC and increased remyelination compared to the lateral part.

\subsubsection{ESTIM failed to exert effects on remyelination or oligodendrocyte populations}

No effect was observed in electrically stimulated animals compared to sham treated animals if comparing the demyelination score in LFB-PAS stainings or the oligodendrocyte populations in the corpus callosum $(3.2 .2 .2 .1,3.2 .2 .2 .2)$. Albeit, the OPC (Olig $2^{+} \mathrm{NogoA}^{-}$ $\mathrm{BrdU}^{+}$) in the lateral corpus callosum of sham animals showed a significant decrease after ten days compared to five days of remyelination, while the OPC numbers of ESTIM animals showed no significant effect. This might indicate a slight delay of OPC differentiation in ESTIM animals, which, however, did not influence the remyelination of the corpus callosum. In fact, only ESTIM animals demonstrated a significant decrease in the DM score between five and ten days of remyelination. Furthermore, no effect on proliferating OPC or mature oligodendrocytes (NogoA ${ }^{+} \mathrm{BrdU}^{+}$) was uncovered by comparing the two experimental groups directly. Interestingly, the overall amount of proliferating OPC was very low for both time points and treatment conditions, with many mice not showing any $\mathrm{Olig}^{+} \mathrm{NogoA}^{-} \mathrm{BrdU}^{+}$cells. Since remyelination could be observed, this indicates that the proliferation of OPC necessary for remyelination was either performed outside the corpus callosum or started already during the phase of Cuprizone exposure and therefore before we injected the mice with BrdU.

Moreover, the duration of electrical stimulation for either five or ten days seemed to have no influence as well, since both time points displayed equal results. Similar to the previous experiment on focal cortical demyelination in rats, a change in the stimulation protocol has to be considered. Neuronal activity is a crucial factor for increased OPC proliferation/differentiation and myelin production as observed in the literature (Bergles et al., 2000; Gary et al., 2012; Gibson et al., 2014; Ishibashi et al., 2006). To improve the ability of our electrical stimulation protocols to influence neuronal activity, an increase of stimulation intensity, as discussed before, or an alteration of the firing pattern should be investigated. 
However, one has to be careful in choosing such protocols, since not only can heat-induced lesions occur by increasing the stimulation intensity (Liebetanz et al., 2009), but the artificial increase of axonal firing could also lead to the generation of epileptic seizures (Norwood et al., 2011). 


\section{Part III - Discussion}

Transcranial electrical stimulation in healthy human subjects 


\subsection{Transcranial electrical stimulation in healthy human subjects}

With the "theta burst" protocol (TBS), Huang and colleagues established an rTMS stimulation protocol, which was able to either facilitate or inhibit cortical excitability depending on the TBS pattern (Huang et al., 2005). Since the application of rTMS can be very uncomfortable for the recipient and bears elevated risks of inducing epileptic seizures, we tried to replicate the results observed by Huang et al. by using safer and more pleasant high frequency, high intensity tACS protocols.

\subsubsection{Differences in electric field strength and orientation may cause lack of effects on cortical excitability by eTBS protocols}

Huang et al. demonstrated in 2005 that the application of rTMS based iTBS and CTBS led to facilitation and inhibition of MEP responses for up to one hour, respectively (Huang et al., 2005). In our study, however, the stimulation of healthy participants with tACS based eTBS protocols did not evoke any changes in MEP responses. Furthermore, increasing the stimulation intensity from 5 to $10 \mathrm{~mA}$ and prolonging the sinusoidal burst duration from 1 to 5 ms did not induce the expected effects on MEP amplitudes either. Although we used the same three stimulation patterns (eiTBS, eimTBS and ecTBS), an obvious difference between rTMS and tACS theta burst stimulation is the pulse intensity. While TMS pulses feature field strengths of about $100 \mathrm{mV} / \mathrm{mm}$ for very brief periods, the peak-to-baseline amplitudes of a 5 $\mathrm{mA}$ eTBS sinusoidal waveform for instance only reaches electric fields of up to $1.35 \mathrm{mV} / \mathrm{mm}$ in the cortical area, as indicated by a computational model of electric field distribution performed by A. Opitz (fig. 5 in Kunz et al., 2017). However, electric fields in the range of $1.35 \mathrm{mV} / \mathrm{mm}$ might already be sufficient to modulate transmembrane potentials in neurons and therefore increase the chance of axonal firing, as indicated by Deans and colleagues in 2007 (Deans et al., 2007). Yet, Deans et al. only used frequencies of up to $100 \mathrm{~Hz}$ and no experiments were performed in the kilo-hertz range. While a single pulse of $1.35 \mathrm{mV} / \mathrm{mm}$ may not modulate the neuronal membrane potential drastically, we hypothesized that a continuous application of sinusoidal stimulation with $5 \mathrm{kHz}$ might lead to a summation of those minor effects, resulting in changes of neuronal excitability. In this line, Chaieb and colleagues induced a lasting increase of MEP amplitudes using $1 \mathrm{~mA}$ tACS at $5 \mathrm{kHz}$, demonstrating the effectiveness of $5 \mathrm{kHz}$ tACS in modulating membrane potentials (Chaieb et al., 2011). Moreover, Gildemeister described in 1943 that the voltage threshold required for nerve excitation can be decreased by continuous application of alternating currents in the $\mathrm{kHz}$ range (Gildemeister, 1943). Additionally, a recent study discovered that rat neuronal cultures are able to evoke action potentials in response to electrical fields once every $100 \mu \mathrm{s}$ (Stern et al., 2015). In conjunction with the data obtained by Testa-Silva et al. in 2014, demonstrating the ability of human pyramidal neurons to regulate action potential firing with 
sub-millisecond precision and verifying that human cortical neurons are able to track high frequency inputs more efficiently than rodent cells (Testa-Silva et al., 2014), it can be assumed that human cortical neurons should be able to respond to frequencies of at least 10 $\mathrm{kHz}$. However, despite the aforementioned observations, no modulation of MEP amplitudes was observed for any eTBS protocols compared to sham.

A further difference between rTMS and tACS pulses is the way the electric field passes through the cortical layers. The TMS coil, held tangentially to the scull, induces an electric field which either passes through the cortex in a posterior-anterior (PA) or in an anterior-posterior (AP) direction, depending on the exact coil orientation. A PA directed current would first activate cortical layer 1 before continuing to layer 6 , therefore depolarizing the soma of pyramidal tract cells, while an AP directed current would most likely first activate cortical layer 6 and passing through to layer 1, thus leading to a soma-hyperpolarizing and dendrite-depolarizing effect on pyramidal tract cells (Jefferys, 1981; Sommer et al., 2013). Alternating currents, on the other hand, would interact with PA- as well as AP-oriented structures and thus lack the direction specificity of direct currents. Therefore, to achieve higher direction specificity, a tDCS offset in combination with the eTBS protocols could be considered for future experiments.

\subsubsection{Safe application of $10 \mathrm{~mA}$ eTBS but high interindividual variability}

High pulse electric stimulation can lead to painful sensations on the skin (Merton and Morton, 1980). In addition to previous studies demonstrating the safe application of 1-2 mA tACS in the lower $\mathrm{kHz}$ range (Chaieb et al., 2011; Turi et al., 2013), Cuellar and colleagues observed in 2013 that alternating currents in the frequency range of 2-100 kHz applied to dorsal root ganglions of the exposed spinal cord of rats were able to inhibit sensory neurons and therefore indicating a potential way to alleviate pain perception (Cuellar et al., 2013). Based on this, we chose a $5 \mathrm{kHz}$ carrier frequency to reduce pain perception at the skin. Indeed, during the eTBS protocols with the highest applied intensity of $10 \mathrm{~mA}$ only one of ten participants reported a medium-intensity tingling sensation and another participant reported phosphenes. The remaining participants reported only mild tingling sensations, if any. Therefore, it appears that alternating currents of up to $10 \mathrm{~mA}$ can be safely applied with a 5 $\mathrm{kHz}$ frequency.

Although no changes in MEP amplitudes were observed compared to sham, all original eTBS protocols depicted a slight decrease in MEP size over the time course of 60 minutes, if compared to baseline. However, this is most likely caused by the increasingly relaxed state of the participants sitting for more than one hour in a comfortable chair with arm and head rests. The fact that sham stimulation demonstrated the same effect supports this theory. Furthermore, during the second part of the study, we took greater care in keeping the 
level of consciousness of the participants at a constant level by engaging in soft small talk in the breaks during the measurements. This could explain why none of the altered eTBS protocols showed the decrease of MEP size over time as observed during the first study part.

A further factor that may contribute to the lack of observed effects of cortical excitability might be the very high interindividual variability in MEP responses following any eTBS or even sham protocols. In order to minimize the interindividual variability, we standardized each session. This included an age range of healthy volunteers from 18-45 years, an equal number of female/male participants and a standardization of the stimulation range of each eTBS protocol. Furthermore, we conducted the experiments only between 09:00 am and 06:00 pm, using the same laboratory. Nevertheless, many more factors can contribute to a high variation of MEP results between individual subjects and between repeated sessions with the same subject (Hamada et al., 2013; Maeda et al., 2000). Those factors can be, as mentioned before, the awareness level of the subject or the time of day as well as genetic differences like the BDNF polymorphism Val66Met (Ridding and Ziemann, 2010).

Concluding, eTBS proofed to be a method to safely apply high frequency tACS with intensities of up to $10 \mathrm{~mA}$. However, since no effect on cortical plasticity could be observed, further alterations of the stimulation protocols like, e.g., a tDCS offset should be explored in future experiments. 


\section{Summary and conclusion}

\subsection{Influence of autoimmune inflammation on remyelination in Cuprizone- induced demyelination}

The inflammatory and demyelinating disease multiple sclerosis is characterized by infiltration of autoreactive immune cells into the CNS, demyelinated lesions and axonal damage. Remyelination, driven by OPC and mature oligodendrocytes, is believed to be crucial in preventing permanent axonal damage and loss and is primarily found during RRMS. In the chronic phases of the disease, however, remyelination frequently fails. It was discussed, whether the inflammatory milieu, which is usually more pronounced during the relapsing-remitting phase and less pronounced during chronic MS, can impact the remyelination process.

By immunizing mice with $\mathrm{MOG}_{35-55}$ peptide during simultaneous Cuprizone administration, we established a MS mouse model, which features demyelinated lesions in the CNS with peripherally activated, infiltrating, auto-reactive immune cells like $T$ cells, B cells, granulocytes, NK cells and inflammatory monocytes. Accompanying the increased immune cell infiltration and presence of inflammatory mediators, we observed an increased impairment of axonal transport in the lateral corpus callosum of Cup-EAE mice, indicating increased axonal damage. In addition, a decrease in the amount of mature oligodendrocytes was observed in medial area of the corpus callosum at the remyelination time point following EAE induction. However, further investigation of the remyelination of the corpus callosum by myelin specific stainings revealed no changes between animals with and without adaptive inflammation on top of demyelination. Moreover, a more detailed analysis of the density of myelinated fibers by electron microscopy after 4.5 weeks of Cuprizone administration as well as in the stage of remyelination on a Cuprizone-free diet demonstrated no significant difference between the two experimental groups. Hence, the autoimmune response induced by immunization with a myelin peptide seemed to have no effect on myelin production and remyelination in the Cuprizone-induced mouse model of demyelination. Nevertheless, we demonstrated that the Cuprizone-EAE model features important MS hallmarks like CNS infiltrating, auto-reactive immune cells in combination with demyelination and axonal damage. The Cuprizone-EAE model might therefore be a promising tool for future research on inflammatory demyelinating CNS diseases. 


\subsection{Influence of transcranial alternating current stimulation on spontaneous remyelination in in vitro and in vivo models}

The stimulation of remyelination is of foremost importance in demyelinating diseases such as MS, since it is believed to prevent axons from becoming permanently damaged. The differentiation of OPC into mature, myelinating oligodendrocytes is a crucial step in the process of remyelination. As indicated by recent studies, the non-invasive application of electrical currents could prove to be a promising therapeutic tool to stimulate OPC differentiation, remyelination and nerve fiber regeneration. In the frame of the present work, we developed stimulation protocols for in vitro and in vivo experiments. Initially, we applied $10 \mathrm{~mA}, 10 \mathrm{~Hz}$ alternating currents to oligodendrocyte cultures as well as mixed cortical cultures to evaluate the impact of electrical stimulation on cytotoxicity, myelin production and myelination of axonal fibers. No change in the number of $\mathrm{O}^{+}$cells was observed in ESTIM treated oligodendrocyte cultures, indicating a nontoxic application of electrical fields. Similarly, the amount of $\mathrm{O}^{+} \mathrm{MBP}^{+}$co-labeled cells remained unchanged. Thus, no increase of myelin production was observed in the absence of neurons, which is in line with the current literature. Although the amount of cytotoxicity in electrically stimulated mixed cortical cultures, evaluated by analysis of LDH levels in the cell cultures, did not differ significantly from untreated cultures, the density of myelinated fibers remained unchanged after electrical stimulation as well. Therefore, the effect observed in the literature of increased $\mathrm{MBP}^{+}$ oligodendrocytes after electrical stimulation with $10 \mathrm{~mA}, 10 \mathrm{~Hz}$ biphasic currents could not be replicated in the present experimental setup.

Subsequent experiments were performed on a rat model of focal, cortical demyelination and on a mouse model of Cuprizone-induced, chronic demyelination with impaired remyelination. Due to the lack of safety protocols for the novel method of transcranial alternating current stimulation in rodent models of CNS demyelination, we chose a less intense stimulation protocol of $100 \mu \mathrm{A}$ at $10 \mathrm{~Hz}$ for our in vivo experiments. Indeed, we were able to apply the electrical currents in a safe and mostly non-invasive way, without provoking adverse effects such as heat-induced brain lesions or epileptic seizures. However, analysis of the lesion formation and remyelination by MBP immunohistochemistry revealed a minimal increase of the demyelinated area at five and seven days post injection in ESTIM treated rats compared to sham animals. This effect might be caused by increased myelin clearance by microglia. Apart from that, no significant differences in the size of the demyelinated area, the oligodendrocyte populations or the oligodendrocyte proliferation were observed when comparing ESTIM and sham treated animals. Similar results were obtained in the chronic Cuprizone model, yielding no effect of electrical stimulation on corpus callosum remyelination, oligodendrocyte populations or cell proliferation. Since it is believed that most beneficial effects of electrical stimulation in terms of remyelination are mediated through 
neuronal activity, the most plausible reason for the observed results is the inability of the chosen stimulation protocol to improve neuronal activity. An increase of the stimulation intensity, for instance, could prove to be more efficient. However, increasing the stimulation intensity or prolonging the stimulation protocol could lead to severe adverse effects. Therefore, future experiments should explore safety protocols.

\subsection{Transcranial electrical stimulation in healthy human subjects}

A number of transcranial stimulation protocols were shown to modulate neuronal excitability. The TBS protocols performed by Huang et al. in 2005, for instance, demonstrated inhibitory and excitatory effects on cortical excitability (Huang et al., 2005). However, the application of TBS, as a form of rTMS, is uncomfortable for subjects and bears elevated risks in inducing epileptic seizures. Here, we evaluated the feasibility of an electrical form of TBS, which utilizes high intensity, high frequency alternating currents in a cross-over, double-blind clinical study on healthy human subjects.

The original iTBS and cTBS protocols facilitated and inhibited MEP responses for up to one hour post stimulation, respectively. While we based our eTBS protocols on the original TBS protocols by Huang et al., no lasting effects on MEP amplitudes were observed for any $5 \mathrm{~mA}$ eTBS protocol compared to sham stimulation. Similarly, the continuous application of $10 \mathrm{~mA}$ ecTBS with a sinusoidal burst duration of either 1 or $5 \mathrm{~ms}$ produced no lasting effects on motor responses. An obvious difference between the original TBS and eTBS protocols, which may explain the lack of effects on cortical excitability, is the stimulation intensity. Whereas magnetic TBS features very short impulses with intensities of about $100 \mathrm{mV} / \mathrm{mm}$, we observed current intensities of approximately $1.35 \mathrm{mV} / \mathrm{mm}$ during $5 \mathrm{~mA}$ eTBS (Kunz et al., 2017). Nevertheless, based on the summation principal of Gildemeister (Gildemeister, 1943), we expected that a continuous stimulation with alternating currents might result in an accumulation of charges, ultimately high enough to produce changes in neuronal excitability. Another important difference between magnetic and alternating electric stimulation is the directionality of the applied currents. While electric fields induced by magnetic coils passes through the skull in either AP or PA direction, alternating currents would, by nature, always alternate between both direction during the stimulation and therefore effect pyramidal tract cells in different ways. To achieve higher direction specificity, we propose the use of a tDCS offset for future experiments.

Finally, despite the lack of effect on MEP modulation, eTBS demonstrated to be a safe and non-invasive way to apply high frequency, high intensity alternating currents to the human brain. 


\section{Bibliography}

Ahlgren, C., Odén, A., and Lycke, J. (2011). High nationwide prevalence of multiple sclerosis in Sweden. 17, 901-908. doi:10.1177/1352458511403794.

Al-Majed, a a, Neumann, C. M., Brushart, T. M., and Gordon, T. (2000). Brief electrical stimulation promotes the speed and accuracy of motor axonal regeneration. J. Neurosci. 20, 2602-8. Available at: http://www.ncbi.nlm.nih.gov/pubmed/10729340.

Albert, M., Barrantes-freer, A., Lohrberg, M., Antel, J. P., Prineas, J. W., Palkovits, M., et al. (2016). Synaptic Pathology in the Cerbellar Dentate Nucleus in Chronic Multiple Sclerosis. Brain Pathol. 8472.

Alekseichuk, I., Turi, Z., Lara, G. A. De, Antal, A., and Paulus, W. (2016). Spatial Working Memory in Humans Depends on Theta and High Gamma Synchronization in the Prefrontal Cortex. Curr. Biol., 1-9. doi:10.1016/j.cub.2016.04.035.

Antal, A., and Paulus, W. (2013). Transcranial alternating current stimulation (tACS). Front. Hum. Neurosci. 7, 317. doi:10.3389/fnhum.2013.00317.

Barker, A. T., Jalinous, R., and Freeston, I. L. (1985). Non-Invasive Magnetic Stimulation of Human Motor Cortex. Lancet 325, 1106-1107. doi:10.1016/S0140-6736(85)92413-4.

Barnett, M. H., and Prineas, J. W. (2004). Relapsing and Remitting Multiple Sclerosis: Pathology of the Newly Forming Lesion. Ann. Neurol. 55, 458-468. doi:10.1002/ana.20016.

Barres, B. a, and Raff, M. C. (1993). Proliferation of oligodendrocyte precursor cells depends on electrical activity in axons. Group 361, 258-60. doi:10.1038/361258a0.

Beirowski, B., Babetto, E., Golden, J., Chen, Y.-J., Yang, K., Gross, R., et al. (2014). Metabolic regulator LKB1 plays a crucial role in Schwann cell- mediated axon maintenance Bogdan. Nat. Neurosci. 17, 1351-1361. doi:10.1038/nn.3809.

Ben-Nun, A., Wekerle, H., and Cohen, I. R. (1981). The rapid isolation of clonable antigenspecific T lymphocyte lines capable of mediating autoimmune encephalomyelitis. Eur. J. Immunol. 11, 195-199. doi:10.1002/eji.1830110307.

Bengtsson, S. L., Nagy, Z., Skare, S., Forsman, L., Forssberg, H., and Ullén, F. (2005). Extensive piano practicing has regionally specific effects on white matter development. Nat. Neurosci. 8, 1148-50. doi:10.1038/nn1516. 
Bergles, D. E., Diamond, J. S., and Jahr, C. E. (1999). Clearance of glutamate inside the synapse and beyond. Curr. Opin. Neurobiol. 9, 293-298. doi:10.1016/S09594388(99)80043-9.

Bergles, D. E., Roberts, J. D., Somogyi, P., and Jahr, C. E. (2000). Glutamatergic synapses on oligodendrocyte precursor cells in the hippocampus. Nature 405, 187-91. doi:10.1038/35012083.

Berridge, M., Lipp, P., and Bootman, M. (2000). Signal transduction. The calcium entry pas de deux. Science (80-. ). 287, 1604-5.

Bettelli, E., Pagany, M., Weiner, H. L., Linington, C., Sobel, R. A., and Kuchroo, V. K. (2003). Myelin Oligodendrocyte Glycoprotein-specific T Cell Receptor Transgenic Mice Develop Spontaneous Autoimmune Optic Neuritis. J. Exp. Med. J. Exp. Med 0, 1073-1081. doi:10.1084/jem.20021603.

Bielekova, B., Sung, M.-H., Kadom, N., Simon, R., McFarland, H., and Martin, R. (2004). Expansion and functional relevance of high-avidity myelin-specific CD4+ T cells in multiple sclerosis. J. Immunol. 172, 3893-904. doi:10.4049/JIMMUNOL.172.6.3893.

Billiau, A., and Matthys, P. (2001). Modes of action of Freund's adjuvants in experimental models of autoimmune diseases. J. Leukoc. Biol. 70, 849-860. Available at: http://www.jleukbio.org/content/70/6/849\%5Cnhttp://www.jleukbio.org/content/70/6/849.f ull\%5Cnhttp://www.jleukbio.org/content/70/6/849.full.pdf\%5Cnhttp://www.ncbi.nlm.nih.g ov/pubmed/11739546.

Bø, L., Vedeler, C. a, Nyland, H. I., Trapp, B. D., and Mørk, S. J. (2003). Subpial demyelination in the cerebral cortex of multiple sclerosis patients. J. Neuropathol. Exp. Neurol. 62, 723-732. doi:10.1093/jnen/62.7.723.

Boggio, P., Nunes, A., Rigonatti, S., Nitsche, M., Pascual-Leone, A., and Fregni, F. (2007). Repeated sessions of noninvasive brain DC stimulation is associated with motor function improvement in stroke patients. Restor. Neurol. Neurosci. 25, 123-9.

Booss, J., Esiri, M., Tourtellotte, W., and Mason, D. (1983). Immunohistological analysis of T lymphocyte subsets in the central nervous system in chronic progressive multiple sclerosis. J Neurol Sci 62, 219-32.

Boretius, S., Escher, A., Dallenga, T., Wrzos, C., Tammer, R., Brück, W., et al. (2012). Assessment of lesion pathology in a new animal model of MS by multiparametric MRI and DTI. Neuroimage 59, 2678-88. doi:10.1016/j.neuroimage.2011.08.051. 
Bourke, J. L., Coleman, H. a, Pham, V., Forsythe, J. S., and Parkington, H. C. (2014). Neuronal electrophysiological function and control of neurite outgrowth on electrospun polymer nanofibers are cell type dependent. Tissue Eng. Part A 20, 1089-95. doi:10.1089/ten.TEA.2013.0295.

Bunge, M. B., Bunge, R. P., and Ris, H. (1961). Ultrastructural study of remyelination in an experimental lesion in adult cat spinal cord. J. Biophys. Biochem. Cytol. 10, 67-94. doi:10.1083/jcb.10.1.67.

Butzkueven, H., Emery, B., Cipriani, T., Marriott, M., and Kilpatrick, T. (2006). Endogenous Leukemia Inhibitory Factor Production Limits Autoimmune Demyelination and Oligodendrocyte Loss. Glia 53, 696-703. doi:10.1002/glia.20321.

Campbell, G., Kraytsberg, Y., Krishnan, K., Ohno, N., Ziabreva, I., Reeve, A., et al. (2012). Clonal Expansion of Mitochondrial DNA Deletions in Multiple Sclerosis. Acta Neuropathol. 124, 209-220. doi:10.1007/s00401-012-1001-9.

Cao, Y., Goods, B., Raddassi, K., Nepom, G., Kwok, W., Love, C., et al. (2015). Distinct Inflammatory Profiles of Myelin-Reactive T cells from Patients with Multiple Sclerosis. Sci Transl Med 7. doi:10.1126/scitransImed.aaa8038.

Carlton, W. (1967). Studies on the induction of hydrocephalus and spongy degeneration by cuprizone feeding and attempts to antidote the toxicity. Life Sci 6, 11-9.

Chaieb, L., Antal, A., and Paulus, W. (2011). Transcranial alternating current stimulation in the low kHz range increases motor cortex excitability. Restor. Neurol. Neurosci. 29, 167-175. doi:10.3233/RNN-2011-0589.

Chan, J. R., Jolicoeur, C., Yamauchi, J., Elliot, J., Fawcett, J. W., Ng, B. K., et al. (2006). The polarity protein Par-3 regulates myelination. Science (80-. ). 314, 832-836. doi:00/00/0A/85/ [pii]\r10.1051/medsci/2007233259.

Charles, P., Hernandez, M. P., Stankoff, B., Aigrot, M. S., Colin, C., Rougon, G., et al. (2000). Negative regulation of central nervous system myelination by polysialylatedneural cell adhesion molecule. Proc. Natl. Acad. Sci. U. S. A. 97, 7585-90. doi:10.1073/pnas.100076197.

Cohen, R. I., Marmur, R., Norton, W. T., Mehler, M. F., and Kessler, J. A. (1996). Nerve growth factor and neurotrophin-3 differentially regulate the proliferation and survival of developing rat brain oligodendrocytes. J. Neurosci. 16, 6433-6442. Available at: http://www.ncbi.nlm.nih.gov/pubmed/8815922. 
Coles, A. J., Cox, A., Le Page, E., Jones, J., Trip, S. A., Deans, J., et al. (2006). The window of therapeutic opportunity in multiple sclerosis: Evidence from monoclonal antibody therapy. J. Neurol. 253, 98-108. doi:10.1007/s00415-005-0934-5.

Compston, A., and Coles, A. (2008). Multiple sclerosis. Lancet 372, 1502-1517. doi:10.1016/S0140-6736(08)61620-7.

Cooper, M., Fehniger, T., and Caligiuri, M. A. (2001). The biology of human natural killer-cell subsets. Trends Immunol. 22, 633-640. doi:10.1016/S1471-4906(01)02060-9.

Croxford, A. L., Kurschus, F. C., and Waisman, A. (2011). Mouse models for multiple sclerosis: historical facts and future implications. Biochim Biophys Acta. 1812, 177-83. doi:10.1016/j.bbadis.2010.06.010.

Cuellar, J. M., Alataris, K., Walker, A., Yeomans, D. C., and Antognini, J. F. (2013). Effect of high-frequency alternating current on spinal afferent nociceptive transmission. Neuromodulation 16, 318-327. doi:10.1111/ner.12015.

Dale, D., Boxer, L., and Liles, W. (2008). The phagocytes : neutrophils and monocytes. Blood 112, 935-946. doi:10.1182/blood-2007-12-077917.

Dawson, M. R. L., Polito, A., Levine, J. M., and Reynolds, R. (2003). NG2-expressing glial progenitor cells: An abundant and widespread population of cycling cells in the adult rat CNS. Mol. Cell. Neurosci. 24, 476-488. doi:10.1016/S1044-7431(03)00210-0.

Deans, J. K., Powell, A. D., and Jefferys, J. G. R. (2007). Sensitivity of coherent oscillations in rat hippocampus to AC electric fields. J. Physiol. 583, 555-565. doi:10.1113/jphysiol.2007.137711.

Demerens, C., Stankoff, B., Logak, M., Anglade, P., Allinquant, B., Couraud, F., et al. (1996). Induction of myelination in the central nervous system by electrical activity. Proc. Natl. Acad. Sci. U. S. A. 93, 9887-92. Available at: http://www.pubmedcentral.nih.gov/articlerender.fcgi?artid=38524\&tool=pmcentrez\&rend ertype=abstract.

Dendrou, C. A., Fugger, L., and Friese, M. A. (2015). Immunopathology of multiple sclerosis. Nat. Publ. Gr. 15, 545-558. doi:10.1038/nri3871.

Duchini, A., Govindarajan, S., Santucci, M., Zampi, G., and Hofman, F. (1996). Effects of tumor necrosis factor-alpha and interleukin- 6 on fluid-phase permeability and ammonia diffusion in CNS-derived endothelial cells. J Investig Med 44, 474-82. 
Dugas, J. C., Cuellar, T. L., Scholze, A., Ason, B., Emery, B., Zamanian, J. L., et al. (2011). Dicer1 and miR-219 Are Required for Normal Oligodendrocyte Differentiation and Myelination. Neuron 65, 597-611. doi:10.1016/j.neuron.2010.01.027.

Duncan, I. D., Brower, A., Kondo, Y., Curlee, J. F., and Schultz, R. D. (2009). Extensive remyelination of the CNS leads to functional recovery. 106, 6832-6836. doi:10.1073/pnas.0812500106.

Emery, B., Agalliu, D., Cahoy, J. D., Watkins, T. A., Dugas, J. C., Sara, B., et al. (2009). Identification of Myelin-gene Regulatory Factor as a Critical Transcriptional Regulator Required for CNS Myelination. Cell 138, 172-185. doi:10.1016/j.cell.2009.04.031.

Eriksson, M., Andersen, O., and Runmarker, B. (2003). Long-term follow up of patients with clinically isolated syndromes, relapsing-remitting and secondary progressive multiple sclerosis. 260-274.

Escher, A. (2008). Neuroaxonale Schädigung in experimentellen Modellen der multiplen Sklerose. Dr. Thesis.

Fernández, M., Baldassarro, V. A., Sivilia, S., Giardino, L., and Calzà, L. (2016). Inflammation severely alters thyroid hormone signaling in the central nervous system during experimental allergic encephalomyelitis in rat: Direct impact on OPCs differentiation failure. Glia 64, 1573-1589. doi:10.1002/glia.23025.

Feurra, M., Paulus, W., Walsh, V., and Kanai, R. (2011). Frequency specific modulation of human somatosensory: Cortex. Front. Psychol. 2, 1-6. doi:10.3389/fpsyg.2011.00013.

Fields, R. D. (2008). White matter in learning, cognition and psychiatric disorders. Trends Neurosci. 31, 361-370. doi:10.1016/j.tins.2008.04.001.

Fields, R. D., and Ni, Y. (2010). Nonsynaptic Communication Through ATP Release from Volume-Activated Anion Channels in Axons. Sci. Signal. 3. doi:10.1126/scisignal.2001128.

Fischer, M. T., Sharma, R., Lim, J. L., Haider, L., Frischer, J. M., Drexhage, J., et al. (2012). NADPH oxidase expression in active multiple sclerosis lesions in relation to oxidative tissue damage and mitochondrial injury. Brain 135, 886-899. doi:10.1093/brain/aws012.

Fischer, M. T., Wimmer, I., Höftberger, R., Gerlach, S., Haider, L., Zrzavy, T., et al. (2013). Disease-specific molecular events in cortical multiple sclerosis lesions. Brain 136, 1799-1815. doi:10.1093/brain/awt110. 
Flügel, A., Berkowicz, T., Ritter, T., Labeur, M., Jenne, D. E., Li, Z., et al. (2001). Migratory Activity and Functional Changes of Green Fluorescent Effector Cells before and during Experimental Autoimmune Encephalomyelitis. Immunity 14, 547-560. doi:10.1016/S1074-7613(01)00143-1.

Foote, a. K., and Blakemore, W. F. (2005). Inflammation stimulates remyelination in areas of chronic demyelination. Brain 128, 528-539. doi:10.1093/brain/awh417.

Franklin, R. J. M. (2002). Why does remyelination fail in multiple sclerosis? Nat. Rev. Neurosci. 3, 705-14. doi:10.1038/nrn917.

Frischer, J. M., Bramow, S., Dal-Bianco, A., Lucchinetti, C. F., Rauschka, H., Schmidbauer, M., et al. (2009). The relation between inflammation and neurodegeneration in multiple sclerosis brains. Brain 132, 1175-1189. doi:10.1093/brain/awp070.

Fünfschilling, U., Supplie, L. M., Mahad, D., Boretius, S., Saab, A. S., Edgar, J., et al. (2012). Glycolytic oligodendrocytes maintain myelin and long-term axonal integrity. Nature 485, 517-21. doi:10.1038/nature11007.

Gamboa, O. L., Antal, A., Moliadze, V., and Paulus, W. (2010). Simply longer is not better: reversal of theta burst after-effect with prolonged stimulation. Exp. brain Res. 204, 1817. doi:10.1007/s00221-010-2293-4.

Gandhi, R., Laroni, a, and Weiner, H. (2010). Role of the innate immune system in the pathogenesis of multiple sclerosis. J neuroimmunol 221, 7-14. doi:10.1016/j.jneuroim.2009.10.015.Role.

Gary, D. S., Malone, M., Capestany, P., Houdayer, T., and McDonald, J. W. (2012). Electrical stimulation promotes the survival of oligodendrocytes in mixed cortical cultures. J. Neurosci. Res. 90, 72-83. doi:10.1002/jnr.22717.

Gensert, J. M., and Goldman, J. E. (1997). Endogenous progenitors remyelinate demyelinated axons in the adult CNS. Neuron 19, 197-203. doi:10.1016/S08966273(00)80359-1.

Gibson, E. M., Purger, D., Mount, C. W., Goldstein, A. K., Lin, G. L., Wood, L. S., et al. (2014). Neuronal activity promotes oligodendrogenesis and adaptive myelination in the mammalian brain. Science 344, 1252304. doi:10.1126/science.1252304.

Gildemeister, M. (1943). Untersuchungen über die Wirkung der Mittelfrequenzströme. 247, 366-404. 
Goldschmidt, T., Antel, J., König, F., Brück, W., and Kuhlmann, T. (2009). Remyelination capacity of the MS brain decreases with disease chronicity. Neurology 72, 1914-21.

Goverman, J. (2009). Autoimmune T cell responses in the central nervous system. Immunology 9, 393-407. doi:10.1038/nri2550.Autoimmune.

Gray, E., Thomas, T. L., Betmouni, S., Scolding, N., and Love, S. (2008). Elevated myeloperoxidase activity in white matter in multiple sclerosis. Neurosci. Lett. 444, 195198. doi:10.1016/j.neulet.2008.08.035.

Haider, L., Fischer, M. T., Frischer, J. M., Bauer, J., Höftberger, R., Botond, G., et al. (2011). Oxidative damage in multiple sclerosis lesions. Brain 134, 1914-1924. doi:10.1093/brain/awr128.

Hamada, M., Murase, N., Hasan, A., Balaratnam, M., and Rothwell, J. C. (2013). The role of interneuron networks in driving human motor cortical plasticity. Cereb. Cortex 23, 1593605. doi:10.1093/cercor/bhs147.

Harsan, L.-A., Steibel, J., Zaremba, A., Agin, A., Sapin, R., Poulet, P., et al. (2008). Neurobiology of Disease Recovery from Chronic Demyelination by Thyroid Hormone Therapy: Myelinogenesis Induction and Assessment by Diffusion Tensor Magnetic Resonance Imaging. 28, 14189-14201. doi:10.1523/JNEUROSCI.4453-08.2008.

Hauser, S. L., Chan, J. R., and Oksenberg, J. R. (2013). Multiple sclerosis: Prospects and promise. Ann. Neurol. 74, 317-27. doi:10.1002/ana.24009.

Hawker, K., O'Connor, P., Freedman, M. S., Calabresi, P. A., Antel, J., Simon, J., et al. (2009). Rituximab in patients with primary progressive multiple sclerosis: Results of a randomized double-blind placebo-controlled multicenter trial. Ann. Neurol. 66, 460-471. doi:10.1002/ana.21867.

Hayashi, T., Morimoto, C., Burks, J. S., Kerr, C., and Hauser, S. L. (1988). Dual-label immunocytochemistry of the active multiple sclerosis lesion: Major histocompatibility complex and activation antigens. Ann. Neurol. 24, 523-531. doi:10.1002/ana.410240408.

Hiremath, M. M., Saito, Y., Knapp, G. W., Ting, J. P. Y., Suzuki, K., and Matsushima, G. K. (1998). Microglial/macrophage accumulation during cuprizone-induced demyelination in C57BL/6 mice. J. Neuroimmunol. 92, 38-49. doi:10.1016/S0165-5728(98)00168-4. 
Hofstetter, H. H., Shive, C. L., and Forsthuber, T. G. (2002). Pertussis Toxin Modulates the Immune Response to Neuroantigens Injected in Incomplete Freund's Adjuvant: Induction of Th1 Cells and Experimental Autoimmune Encephalomyelitis in the Presence of High Frequencies of Th2 Cells. J. Immunol. 169, 117-125. doi:10.4049/jimmunol.169.1.117.

Hohlfeld, R. (2007). Does inflammation stimulate remyelination? J. Neurol. 254, 47-54. doi:10.1007/s00415-007-1009-6.

Huang, J., Zhang, Y., Lu, L., Hu, X., and Luo, Z. (2013). Electrical stimulation accelerates nerve regeneration and functional recovery in delayed peripheral nerve injury in rats. Eur. J. Neurosci. 38, 3691-701. doi:10.1111/ejn.12370.

Huang, Y. M., Xiao, B. G., Ozenci, V., Kouwenhoven, M., Teleshova, N., Fredrikson, S., et al. (1999). Multiple sclerosis is associated with high levels of circulating dendritic cells secreting pro-inflammatory cytokines. J. Neuroimmunol. 99, 82-90. doi:10.1016/S01655728(99)00106-X.

Huang, Y. Z., Chen, R. S., Rothwell, J. C., and Wen, H. Y. (2007). The after-effect of human theta burst stimulation is NMDA receptor dependent. Clin. Neurophysiol. 118, 10281032. doi:DOI 10.1016/j.clinph.2007.01.021.

Huang, Y. Z., Edwards, M. J., Rounis, E., Bhatia, K. P., and Rothwell, J. C. (2005). Theta burst stimulation of the human motor cortex. Neuron 45, 201-206. doi:10.1016/j.neuron.2004.12.033.

Hussain, R., Ghoumari, A. M., Bielecki, B., Steibel, J., Boehm, N., Liere, P., et al. (2013). The neural androgen receptor: $A$ therapeutic target for myelin repair in chronic demyelination. Brain 136, 132-146. doi:10.1093/brain/aws284.

Ioannidou, K., Anderson, K. I., Strachan, D., Edgar, J. M., and Barnett, S. C. (2012). Timelapse imaging of the dynamics of CNS glial-axonal interactions in vitro and ex vivo. PLoS One 7. doi:10.1371/journal.pone.0030775.

Irvine, K. A., and Blakemore, W. F. (2008). Remyelination protects axons from demyelination-associated axon degeneration. Brain 131, 1464-1477. doi:10.1093/brain/awn080.

Ishibashi, T., Dakin, K. A., Stevens, B., Lee, P. R., Kozlov, S. V., Stewart, C. L., et al. (2006). Astrocytes promote myelination in response to electrical impulses. Neuron 49, 823-832. doi:10.1016/j.neuron.2006.02.006. 
Jahanshahi, A., Schonfeld, L., Janssen, M. L. F., Hescham, S., Kocabicak, E., Steinbusch, H. W. M., et al. (2013). Electrical stimulation of the motor cortex enhances progenitor cell migration in the adult rat brain. Exp. brain Res. 231, 165-77. doi:10.1007/s00221013-3680-4.

Jeffery, N., and Blakemore, W. (1995). Remyelination of mouse spinal cord axons demyelinated by local injection of lysolecithin. J Neurocytol 24, 775-81.

Jefferys, J. G. R. (1981). Influence of electric fields on the excitability of granule cells in guinea-pig hippocampal slices. J Physiol, 143-152.

Jürgens, T., Jafari, M., Kreutzfeldt, M., Bahn, E., Brück, W., Kerschensteiner, M., et al. (2016). Reconstruction of single cortical projection neurons reveals primary spine loss in multiple sclerosis. Brain 139, 39-46. doi:10.1093/brain/awv353.

Kabat, E. A., Wolf, A., Bezer, A. E., and Murray, J. P. (1951). Studies on Acute Disseminated Encephalomyelitis Produced Experimentally in Rhesus Monkeys. J. Exp. Med. 93, 615633. Available at:

http://www.ncbi.nlm.nih.gov/pmc/articles/PMC2136070/\%5Cnhttp://www.ncbi.nlm.nih.go v/pmc/articles/PMC2136070/pdf/615.pdf.

Keirstead, H. S., and Blakemore, W. F. (1997). Identification of post-mitotic oligodendrocytes incapable of remyelination within the demyelinated adult spinal cord. J. Neuropathol. Exp. Neurol. 56, 1191-1201. Available at: http://www.ncbi.nlm.nih.gov/pubmed/9370229.

Kerschensteiner, M., Gallmeier, E., Behrens, L., Leal, V., Misgeld, T., Klinkert, W. E., et al. (1999). Activated human T cells, B cells, and monocytes produce brain-derived neurotrophic factor in vitro and in inflammatory brain lesions: a neuroprotective role of inflammation? J. Exp. Med. 189, 865-870. doi:10.1084/jem.189.5.865.

Kerschensteiner, M., Stadelmann, C., Dechant, G., Wekerle, H., and Hohlfeld, R. (2003). Neurotrophic cross-talk between the nervous and immune systems: Implications for neurological diseases. Ann. Neurol. 53, 292-304. doi:10.1002/ana.10446.

Khedr, E. M., Ahmed, M. A., Fathy, N., and Rothwell, J. C. (2005). Therapeutic trial of repetitive transcranial magnetic stimulation after acute ischemic stroke. Neurology 65, 466-468. doi:10.1212/01.wnl.0000173067.84247.36.

Kim, J. H., Budde, M. D., Liang, H. F., Klein, R. S., Russell, J. H., Cross, A. H., et al. (2006). Detecting axon damage in spinal cord from a mouse model of multiple sclerosis. Neurobiol. Dis. 21, 626-632. doi:10.1016/j.nbd.2005.09.009. 
Kirson, E. D., Dbalý, V., Tovarys, F., Vymazal, J., Soustiel, J. F., Itzhaki, A., et al. (2007). Alternating electric fields arrest cell proliferation in animal tumor models and human brain tumors. Proc. Natl. Acad. Sci. U. S. A. 104, 10152-7. doi:10.1073/pnas.0702916104.

Kirson, E. D., Gurvich, Z., Schneiderman, R., Dekel, E., Itzhaki, A., Wasserman, Y., et al. (2004). Disruption of Cancer Cell Replication by Alternating Electric Fields. Cancer Res. 64, 3288-3295.

Kirson, E. D., Schneiderman, R. S., Dbalý, V., Tovarys, F., Vymazal, J., Itzhaki, A., et al. (2009). Chemotherapeutic treatment efficacy and sensitivity are increased by adjuvant alternating electric fields (TTFields). BMC Med. Phys. 9, 1. doi:10.1186/1756-6649-9-1.

Kuhlmann, T., Lingfeld, G., Bitsch, A., Schuchardt, J., and Brück, W. (2002). Acute axonal damage in multiple sclerosis is most extensive in early disease stages and decreases over time. Brain 125, 2202-2212. doi:10.1093/brain/awf235.

Kuhlmann, T., Ludwin, S., Prat, A., Antel, J., Brück, W., and Lassmann, H. (2017). An updated histological classification system for multiple sclerosis lesions. Acta Neuropathol. 133, 13-24. doi:10.1007/s00401-016-1653-y.

Kuhlmann, T., Remington, L., Maruschak, B., Owens, T., and Brück, W. (2007). Nogo-A is a reliable oligodendroglial marker in adult human and mouse CNS and in demyelinated lesions. J. Neuropathol. Exp. Neurol. 66, 238-246. doi:10.1097/01.jnen.0000248559.83573.71.

Kunz, P., Antal, A., Hewitt, M., Neef, A., Opitz, A., and Paulus, W. (2017). 5 kHz Transcranial Alternating Current Stimulation: Lack of Cortical Excitability Changes When Grouped in a Theta Burst Pattern. Front. Hum. Neurosci. 10, 1-8. doi:10.3389/fnhum.2016.00683.

Kutzelnigg, A., Lucchinetti, C. F., Stadelmann, C., Brück, W., Rauschka, H., Bergmann, M., et al. (2005). Cortical demyelination and diffuse white matter injury in multiple sclerosis. Brain 128, 2705-2712. doi:10.1093/brain/awh641.

Lagumersindez-Denis, N., Wrzos, C., Mack, M., Winkler, A., van der Meer, F., Reinert, M. C., et al. (2017). Differential contribution of immune effector mechanisms to cortical demyelination in multiple sclerosis. Acta Neuropathol. doi:10.1007/s00401-017-1706-x.

Lassmann, H., and Bradl, M. (2017). Multiple sclerosis : experimental models and reality. Acta Neuropathol. 133, 223-244. doi:10.1007/s00401-016-1631-4. 
Lassmann, H., Brück, W., and Lucchinetti, C. F. (2007). The immunopathology of multiple sclerosis: An overview. Brain Pathol. 17, 210-218. doi:10.1111/j.17503639.2007.00064.x.

Di Lazzaro, V., Pilato, F., Saturno, E., Oliviero, A., Dileone, M., Mazzone, P., et al. (2005). Theta-burst repetitive transcranial magnetic stimulation suppresses specific excitatory circuits in the human motor cortex. J. Physiol. 565, 945-50. doi:10.1113/jphysiol.2005.087288.

Ledeen, R. W., and Chakraborty, G. (1998). Cytokines, signal transduction, and inflammatory demyelination: Review and hypothesis. Neurochem. Res. 23, 277-289. doi:10.1023/A:1022493013904.

Lee, Y., Morrison, B. M., Li, Y., Lengacher, S., Farah, M. H., Hoffman, P. N., et al. (2012). Oligodendroglia metabolically support axons and contribute to neurodegeneration. Nature 487, 443-448. doi:10.1038/nature11314.

Lefaucheur, J.-P., Andre-Obadia, N., Antal, A., Ayache, S. S., Baeken, C., Benninger, D. H., et al. (2014). Evidence-based guidelines on the therapeutic use of repetitive transcranial magnetic stimulation (rTMS). Clin. Neurophysiol. 125, 2150-2206. doi:10.1016/j.clinph.2014.05.021.

Lenz, M., Platschek, S., Priesemann, V., Becker, D., Willems, L. M., Ziemann, U., et al. (2014). Repetitive magnetic stimulation induces plasticity of excitatory postsynapses on proximal dendrites of cultured mouse CA1 pyramidal neurons. Brain Struct. Funct. 220, 3323-3337. doi:10.1007/s00429-014-0859-9.

Li, L., El-Hayek, Y. H., Liu, B., Chen, Y., Gomez, E., Wu, X., et al. (2008). Direct-current electrical field guides neuronal stem/progenitor cell migration. Stem Cells 26, 2193-200. doi:10.1634/stemcells.2007-1022.

Liebetanz, D., Koch, R., Mayenfels, S., König, F., Paulus, W., and Nitsche, M. a (2009). Safety limits of cathodal transcranial direct current stimulation in rats. Clin. Neurophysiol. 120, 1161-7. doi:10.1016/j.clinph.2009.01.022.

Lin, W., Kemper, A., Dupree, J. L., Harding, H. P., Ron, D., and Popko, B. (2006). Interferon$Y$ inhibits central nervous system remyelination through a process modulated by endoplasmic reticulum stress. Brain 129, 1306-1318. doi:10.1093/brain/awl044.

Liu, A., Li, J., Marin-Husstege, M., Kageyama, R., Fan, Y., Gelinas, C., et al. (2006). A molecular insight of Hes5-dependent inhibition of myelin gene expression: old partners and new players. EMBO J. 25, 4833-42. doi:10.1038/sj.emboj.7601352. 
Liu, J., and Casaccia-Bonnefil, P. (2010). Epigenetic regulation of oligodendrocyte identity.

Trends Neurosci. 33, 193-201. doi:10.1016/j.tins.2010.01.007.

Lund, F. E. (2008). Cytokine-producing B lymphocytes-key regulators of immunity. Curr. Opin. Immunol. 20, 332-8. doi:10.1016/j.coi.2008.03.003.

Lundgaard, I., Luzhynskaya, A., Stockley, J. H., Wang, Z., Evans, K. a, Swire, M., et al. (2013). Neuregulin and BDNF Induce a Switch to NMDA Receptor-Dependent Myelination by Oligodendrocytes. PLoS Biol. 11, e1001743. doi:10.1371/journal.pbio.1001743.

Maeda, F., Keenan, J. P., Tormos, J. M., Topka, H., and Pascual-Leone, A. (2000). Interindividual variability of the modulatory effects of repetitive transcranial magnetic stimulation on cortical excitability. Exp. Brain Res. 133, 425-430. doi:10.1007/s002210000432.

Maldonado, P. P., and Angulo, M. C. (2015). Multiple Modes of Communication between Neurons and Oligodendrocyte Precursor Cells. Neurosci. 21, 266-276. doi:10.1177/1073858414530784.

Malone, M., Gary, D., Yang, I. H., Miglioretti, A., Houdayer, T., Thakor, N., et al. (2013). Neuronal activity promotes myelination via a cAMP pathway. Glia 61, 843-54. doi:10.1002/glia.22476.

Mangin, J.-M., Li, P., Scafidi, J., and Gallo, V. (2012). Experience-dependent regulation of NG2 progenitors in the developing barrel cortex. Nat Neu 15, 1192-1194. doi:10.1038/nn.3190.

Marik, C., Felts, P. A., Bauer, J., Lassmann, H., and Smith, K. J. (2007). Lesion genesis in a subset of patients with multiple sclerosis: A role for innate immunity? Brain 130, 2800 2815. doi:10.1093/brain/awm236.

Martins, T. B., Rose, J. W., Jaskowski, T. D., Wilson, A. R., Husebye, D., Seraj, H. S., et al. (2011). Analysis of proinflammatory and anti-inflammatory cytokine serum concentrations in patients with multiple sclerosis by using a multiplexed immunoassay. Am. J. Clin. Pathol. 136, 696-704. doi:10.1309/AJCP7UBK8IBVMVNR.

Mason, J. L., Toews, A., Hostettler, J. D., Morell, P., Suzuki, K., Goldman, J. E., et al. (2004). Oligodendrocytes and progenitors become progressively depleted within chronically demyelinated lesions. Am. J. Pathol. 164, 1673-82. doi:10.1016/S0002-9440(10)637261. 
Matsushima, G. K., and Morell, P. (2001). The neurotoxicant, cuprizone, as a model to study demyelination and remyelination in the central nervous system. Brain Pathol. 11, 107116. doi:10.1111/j.1750-3639.2001.tb00385.x.

McLean, N. a, Popescu, B. F., Gordon, T., Zochodne, D. W., and Verge, V. M. K. (2014). Delayed nerve stimulation promotes axon-protective neurofilament phosphorylation, accelerates immune cell clearance and enhances remyelination in vivo in focally demyelinated nerves. PLoS One 9, e110174. doi:10.1371/journal.pone.0110174.

Mendel, I., de Rosbo, N. K., and Ben-Nun, A. (1995). A myelin oligodendrocyte glycoprotein peptide induces typical chronic experimental autoimmune encephalomyelitis in $\mathrm{H}-2 \mathrm{~b}$ mice: Fine specificity and $T$ cell receptor $V$ beta expression of encephalitogenic $T$ cells. Eur. J. Immunol. 25, 1951-1959. doi:10.1002/eji.1830250723.

Menn, B., Garcia-Verdugo, J. M., Yaschine, C., Gonzalez-Perez, O., Rowitch, D., and Alvarez-Buylla, A. (2006). Origin of oligodendrocytes in the subventricular zone of the adult brain. J. Neurosci. 26, 7907-7918. doi:10.1523/JNEUROSCI.1299-06.2006.

Merkler, D., Ernsting, T., Kerschensteiner, M., Brück, W., and Stadelmann, C. (2006). A new focal EAE model of cortical demyelination: multiple sclerosis-like lesions with rapid resolution of inflammation and extensive remyelination. Brain 129, 1972-83. doi:10.1093/brain/awl135.

Merton, P., and Morton, H. (1980). Stimulation of the cerebral cortex in the intact human subject.

Mi, S., Miller, R. H., Lee, X., Scott, M. L., Shulag-Morskaya, S., Shao, Z., et al. (2005). LINGO-1 negatively regulates myelination by oligodendrocytes. Nat. Neurosci. 8, 745751. doi:10.1038/nn1460.

Minagar, A., and Alexander, J. S. (2003). Blood-brain barrier disruption in multiple sclerosis. Mulitple Scler. 9, 540-549. doi:10.1191/1352458503ms965oa.

Mizoguchi, A., and Bhan, A. K. (2006). A case for regulatory B cells. J. Immunol. 176, 70510. doi:10.4049/jimmunol.176.2.705.

Moalem, G., Gdalyahu, a, Shani, Y., Otten, U., Lazarovici, P., Cohen, I. R., et al. (2000). Production of neurotrophins by activated T cells: implications for neuroprotective autoimmunity. J. Autoimmun. 15, 331-345. doi:10.1006/jaut.2000.0441. 
Moliadze, V., Antal, A., and Paulus, W. (2010). Boosting brain excitability by transcranial high frequency stimulation in the ripple range. J Physiol 588, 4891-4904. doi:10.1113/jphysiol.2010.196998.

Moll, N. M., Rietsch, A. M., Ransohoff, A. J., Cossoy, M. B., Huang, D., Eichler, F. S., et al. (2008). Cortical demyelination in PML and MS. Neurology, 336-337.

Morace, R., Di Gennaro, G., Quarato, P., D'Aniello, A., Mascia, A., Grammaldo, L., et al. (2016). Deep brain stimulation for intractabile epilepsy. J. Neurosurg. Sci. 60, 189-198.

Morell, P., Barrett, C. V, Mason, J. L., Toews, A. D., Hostettler, J. D., Knapp, G. W., et al. (1998). Gene expression in brain during cuprizone-induced demyelination and remyelination. Mol. Cell. Neurosci. 12, 220-7. doi:10.1006/mcne.1998.0715.

Morell, P., and Norton, W. (1980). Myelin. Sci Am 242, 88-90.

Morimoto, T., Yasuhara, T., Kameda, M., Baba, T., Kuramoto, S., Kondo, A., et al. (2011). Striatal stimulation Nurtures endogenous neurogenesis and angiogenesis in chronicphase ischemic stroke rats. Cell Transplant. 20, 1049-1064. doi:10.3727/096368910X544915.

Morrison, B. M., Lee, Y., and Rothstein, J. D. (2013). Oligodendroglia metabolically support axons and maintain structural integrity. Trends Cell Biol 23, 1-17. doi:10.1016/j.tcb.2013.07.007.

Mosley, K., and Cuzner, M. L. (1996). Receptor-mediated phagocytosis of myelin by macrophages and microglia: Effect of opsonization and receptor blocking agents. Neurochem. Res. 21, 481-487. doi:10.1007/BF02527713.

Muthuraman, M., Deuschl, G., Koirala, N., Riedel, C., Volkmann, J., and Groppa, S. (2017). Effects of DBS in parkinsonian patients depend on the structural integrity of frontal cortex. Sci. Rep. 7. doi:10.1038/srep43571.

Nait-Oumesmar, B., Decker, L., Lachapelle, F., Avellana-Adalid, V., Bachelin, C., and BaronVan Evercooren, a. (1999). Progenitor cells of the adult mouse subventricular zone proliferate, migrate and differentiate into oligodendrocytes after demyelination. Eur. J. Neurosci. 11, 4357-4366. doi:10.1046/j.1460-9568.1999.00873.x.

Nathan, B. Y. C. F., Murray, H. W., Wiebe, M. E., and Rubin, B. Y. (1983). Identification of interferon-3 as the lymphokine that activates human macrophage oxidative metabolism and antimicrobial activity. 158, 670-689. 
Nathan, C., and Ding, A. (2010). Nonresolving Inflammation. Cell 140, 871-882. doi:10.1016/j.cell.2010.02.029.

Ng, B. K., Chen, L., Mandemakers, W., Cosgaya, J. M., and Chan, J. R. (2007). Anterograde Transport and Secretion of Brain-Derived Neurotrophic Factor along Sensory Axons Promote Schwann Cell Myelination. J. Neurosci. 27, 7597-7603. doi:10.1523/JNEUROSCI.0563-07.2007.

Nikić, I., Merkler, D., Sorbara, C., Brinkoetter, M., Kreutzfeldt, M., Bareyre, F. M., et al. (2011). A reversible form of axon damage in experimental autoimmune encephalomyelitis and multiple sclerosis. Nat. Med. 17, 495-9. doi:10.1038/nm.2324.

Nitsche, M., and Paulus, W. (2000). Excitability changes induced in the human motor cortex by weak transcranial direct current stimulation. J. Physiol. 527 Pt 3, 633-9. doi:PHY_1055 [pii].

Noh, N. A., Fuggetta, G., and Manganotti, P. (2015). Theta-burst transcranial magnetic stimulation alters the functional topography of the cortical motor network. Malaysian $\mathrm{J}$. Med. Sci. 22, 36-44.

Norwood, B. A., Bauer, S., Wegner, S., Hamer, H. M., Oertel, W. H., Sloviter, R. S., et al. (2011). Electrical stimulation-induced seizures in rats: A "dose- response" study on resultant neurodegeneration. Epilepsia 52, 109-112. doi:10.1111/j.15281167.2011.03159.x.

Noseworthy, J., Lucchinetti, C., Rodriguez, M., and Weinshenker, B. (2000). Multiple Sclerosis. Nwe Engl. J. Med. 343, 938-952.

O'Reardon, J. P., Solvason, H. B., Janicak, P. G., Sampson, S., Isenberg, K. E., Nahas, Z., et al. (2007). Efficacy and Safety of Transcranial Magnetic Stimulation in the Acute Treatment of Major Depression: A Multisite Randomized Controlled Trial. Biol. Psychiatry 62, 1208-1216. doi:10.1016/j.biopsych.2007.01.018.

Pangburn, M., Ferreira, V., and Cortes, C. (2008). Discrimination between Host and Pathogens by the Complement System. Vaccine 26, I15-I21. doi:10.1016/j.biotechadv.2011.08.021.Secreted.

Pascual-Leone, A., Davey, N. J., Rothwell, J. C., Wassermann, E. M., and Puri., B. K. (2002). Handbook of Transcranial Magnetic Stimulation. J. Psychiatry Neurosci. 28, 406. Available at: http://www.ncbi.nlm.nih.gov/pmc/articles/PMC193984/. 
Patrikios, P., Stadelmann, C., Kutzelnigg, A., Rauschka, H., Schmidbauer, M., Laursen, H., et al. (2006). Remyelination is extensive in a subset of multiple sclerosis patients. Brain 129, 3165-3172. doi:10.1093/brain/awl217.

Pedraza, L., Huang, J. K., and Colman, D. (2009). Disposition of axonal caspr with respect to glial cell membranes: Implications for the process of myelination. J. Neurosci. Res. 87, 3480-3491. doi:10.1002/jnr.22004.

Pelletier, M., Maggi, L., Micheletti, A., Lazzeri, E., Tamassia, N., Cosmi, L., et al. (2010). Evidence for a cross-talk between human neutrophils and Th17 cells Evidence for a cross-talk between human neutrophils and Th17 cells. 115, 335-343. doi:10.1182/blood-2009-04-216085.

di Penta, A., Moreno, B., Reix, S., Fernandez-Diez, B., Villanueva, M., Errea, O., et al. (2013). Oxidative Stress and Proinflammatory Cytokines Contribute to Demyelination and Axonal Damage in a Cerebellar Culture Model of Neuroinflammation. PLoS One 8. doi:10.1371/journal.pone.0054722.

Pikhovych, A., Stolberg, N. P., Jessica Flitsch, L., Walter, H. L., Graf, R., Fink, G. R., et al. (2016). Transcranial Direct Current Stimulation Modulates Neurogenesis and Microglia Activation in the Mouse Brain. Stem Cells Int. 2016, 2715196. doi:10.1155/2016/2715196.

Pogosyan, A., Gaynor, L. D., Eusebio, A., and Brown, P. (2009). Boosting Cortical Activity at Beta-Band Frequencies Slows Movement in Humans. Curr. Biol. 19, 1637-1641. doi:10.1016/j.cub.2009.07.074.

Pöllinger, B., Krishnamoorthy, G., Berer, K., Lassmann, H., Bosl, M. R., Dunn, R., et al. (2009). Spontaneous relapsing-remitting EAE in the SJL/J mouse: MOG-reactive transgenic T cells recruit endogenous MOG-specific B cells. J Exp Med 206, 13031316. doi:10.1084/jem.20090299.

Popescu, B. F., and Lucchinetti, C. F. (2012). Pathology of demyelinating diseases. Annu Rev Pathol 7, 185-217. doi:10.1146/annurev-pathol-011811-132443.

Prayoonwiwat, N., and Rodriguez, M. (1993). The potential for oligodendrocyte proliferation during demyelinating disease. 55-63.

Raff, M. C., Lillien, L., Richardson, W. D., Burne, J., and Noble, M. (1988). Platelet-derived growth factor from astrocytes drives the clock that times oligodendrocyte development in culture. Nature 336, 562-565. doi:10.1038/333562a0. 
Ridding, M. C., and Ziemann, U. (2010). Determinants of the induction of cortical plasticity by non-invasive brain stimulation in healthy subjects. J. Physiol. 588, 2291-304. doi:10.1113/jphysiol.2010.190314.

Rothkegel, H., Sommer, M., and Paulus, W. (2010). Breaks during 5 Hz rTMS are essential for facilitatory after effects. Clin. Neurophysiol. 121, 426-430. doi:10.1016/j.clinph.2009.11.016.

Rumble, J. M., Huber, A. K., Krishnamoorthy, G., Srinivasan, A., Giles, D. A., Zhang, X., et al. (2015). Neutrophil-related factors as biomarkers in EAE and MS. J. Exp. Med. 212, 23-35. doi:10.1084/jem.20141015.

Saadoun, S., Waters, P., Bell, B. A., Vincent, A., Verkman, A. S., and Papadopoulos, M. C. (2010). Intra-cerebral injection of neuromyelitis optica immunoglobulin $G$ and human complement produces neuromyelitis optica lesions in mice. Brain 133, 349-361. doi:10.1093/brain/awp309.

Saadoun, S., Waters, P., Owens, G. P., Bennett, J. L., Vincent, A., and Papadopoulos, M. C. (2014). Neuromyelitis optica MOG-IgG causes reversible lesions in mouse brain. Acta Neuropathol. Commun. 2, 35. doi:10.1186/2051-5960-2-35.

Scheld, M., Rüther, B. J., Große-Veldmann, R., Ohl, K., Tenbrock, K., Dreymüller, D., et al. (2016). Neurodegeneration Triggers Peripheral Immune Cell Recruitment into the Forebrain. J. Neurosci. 36, 1410-1415. doi:10.1523/JNEUROSCI.2456-15.2016.

Schlegel, A. A., Rudelson, J. J., and Tse, P. U. (2012). White matter structure changes as adults learn a second language. J Cogn Neurosci 24, 1664-1670. doi:10.1162/jocn_a_00240.

Schmidt, T., Awad, H., Slowik, A., Beyer, C., Kipp, M., and Clarner, T. (2013). Regional heterogeneity of cuprizone-induced demyelination: Topographical aspects of the midline of the corpus callosum. J. Mol. Neurosci. 49, 80-88. doi:10.1007/s12031-012-9896-0.

Scholz, J., Klein, M. C., Behrens, T. E. J., and Johansen-berg, H. (2010). Training induces changes in white matter architecture. Nat Neurosci 12, 1370-1371. doi:10.1038/nn.2412.Training.

Serafini, B., Rosicarelli, B., Magliozzi, R., Stigliano, E., and Aloisi, F. (2004). Detection of ectopic B-cell follicles with germinal centers in the meninges of patients with secondary progressive multiple sclerosis. Brain Pathol. 14, 164-174. doi:10.1111/j.1750-

3639.2004.tb00049.x. 
Sherafat, M. A., Heibatollahi, M., Mongabadi, S., Moradi, F., Javan, M., and Ahmadiani, A. (2012). Electromagnetic field stimulation potentiates endogenous myelin repair by recruiting subventricular neural stem cells in an experimental model of white matter demyelination. J. Mol. Neurosci. 48, 144-53. doi:10.1007/s12031-012-9791-8.

Simon, C., Götz, M., and Dimou, L. (2011). Progenitors in the adult cerebral cortex: Cell cycle properties and regulation by physiological stimuli and injury. Glia 59, 869-881. doi:10.1002/glia.21156.

Singh, S., Dallenga, T., Winkler, A., Roemer, S., Maruschak, B., Siebert, H., et al. (2017). Relationship of acute axonal damage, Wallerian degeneration, and clinical disability in multiple sclerosis. J. Neuroinflammation 14, 57. doi:10.1186/s12974-017-0831-8.

Snaidero, N., Möbius, W., Czopka, T., Hekking, L. H. P., Mathisen, C., Verkleij, D., et al. (2014). Myelin membrane wrapping of CNS axons by PI(3,4,5)P3-dependent polarized growth at the inner tongue. Cell 156, 277-290. doi:10.1016/j.cell.2013.11.044.

Sobottka, B., Ziegler, U., Kaech, A., Becher, B., and Goebels, N. (2011). CNS live imaging reveals a new mechanism of myelination: The liquid croissant model. Glia 59, 18411849. doi:10.1002/glia.21228.

Sommer, M., Norden, C., Schmack, L., Rothkegel, H., Lang, N., and Paulus, W. (2013). Opposite optimal current flow directions for induction of neuroplasticity and excitation threshold in the human motor cortex. Brain Stimul. 6, 363-70. doi:10.1016/j.brs.2012.07.003.

Soulika, A. M., Lee, E., McCauley, E., Miers, L., Bannerman, P., and Pleasure, D. (2009). Initiation and progression of axonopathy in experimental autoimmune encephalomyelitis. J. Neurosci. 29, 14965-79. doi:10.1523/JNEUROSCI.3794-09.2009.

Stadelmann, C., Kerschensteiner, M., Misgeld, T., Brück, W., Hohlfeld, R., and Lassmann, H. (2002). BDNF and gp145trkB in multiple sclerosis brain lesions: neuroprotective interactions between immune and neuronal cells? Brain 125, 75-85. doi:10.1093/brain/awf015.

Stadelmann, C., Wegner, C., and Brück, W. (2011). Inflammation, demyelination, and degeneration - Recent insights from MS pathology. Biochim. Biophys. Acta - Mol. Basis Dis. 1812, 275-282. doi:10.1016/j.bbadis.2010.07.007.

Stern, S., Agudelo-Toro, A., Rotem, A., Moses, E., and Neef, A. (2015). Chronaxie measurements in patterned neuronal cultures from rat hippocampus. PLoS One 10, 123. doi:10.1371/journal.pone.0132577. 
Stevens, B., Porta, S., Haak, L. L., Gallo, V., and Fields, R. D. (2002). Adenosine: A neuronglial transmitter promoting myelination in the CNS in response to action potentials. Neuron 36, 855-868. doi:10.1016/S0896-6273(02)01067-X.

Stolt, C. C., Schlierf, A., Lommes, P., Hillgärtner, S., Werner, T., Kosian, T., et al. (2006). SoxD Proteins Influence Multiple Stages of Oligodendrocyte Development and Modulate SoxE Protein Function. Dev. Cell 11, 697-709. doi:10.1016/j.devcel.2006.08.011.

Suzuki, K. (1969). Giant hepatic mitochondria: production in mice fed with cuprizone. Science 163, 81-2. doi:10.1126/science.163.3862.81.

Suzuki, K., and Kikkawa, Y. (1969). Status spongiosus of CNS and hepatic changes induced by cuprizone (biscyclohexanone oxalyldihydrazone). Am. J. Pathol. 54, 307-25. Available at: http://www.pubmedcentral.nih.gov/articlerender.fcgi?artid=2013472\&tool=pmcentrez\&re ndertype $=$ abstract.

Tagge, I., O'Connor, A., Chaudhary, P., Pollaro, J., Berlow, Y., Chalupsky, M., et al. (2016). Spatio-temporal patterns of demyelination and remyelination in the cuprizone mouse model. PLoS One 11, 1-24. doi:10.1371/journal.pone.0152480.

Takahashi, J. L., Giuliani, F., Power, C., Imai, Y., and Yong, V. W. (2003). Interleukin-1 $\beta$ promotes oligodendrocyte death through glutamate excitotoxicity. Ann. Neurol. 53, 588595. doi:10.1002/ana.10519.

Takebayashi, H., Nabeshima, Y., Yoshida, S., Chisaka, O., Ikenaka, K., and Nabeshima, Y. I. (2002). The basic helix-loop-helix factor Olig2 is essential for the development of motoneuron and oligodendrocyte lineages. Curr. Biol. 12, 1157-1163. doi:10.1016/S0960-9822(02)00926-0.

Takeuchi, N., Chuma, T., Matsuo, Y., Watanabe, I., and Ikoma, K. (2005). Repetitive transcranial magnetic stimulation of contralesional primary motor cortex improves hand function after stroke. Stroke 36, 2681-2686. doi:10.1161/01.STR.0000189658.51972.34.

Tallantyre, E. C., Bø, L., Al-Rawashdeh, O., Owens, T., Polman, C. H., Lowe, J. S., et al. (2010). Clinico-pathological evidence that axonal loss underlies disability in progressive multiple sclerosis. Mult. Scler. 16, 406-411. doi:10.1177/1352458510364992. 
Targett, M. P., Sussmant, J., Scoldingt, N., Leary, M. T. O., Compstont, D. A. S., and Blakemore, W. F. (1996). Failure to achieve remyelination of demyelinated rat axons following transplantation of glial cells obtained from the adult human brain. Neuropathol. Appl. Neurobiol. 22, 199-206.

Terney, D., Chaieb, L., Moliadze, V., Antal, A., and Paulus, W. (2008). Increasing Human Brain Excitability by Transcranial High-Frequency Random Noise Stimulation. J. Neurosci. 28, 14147-14155. doi:10.1523/JNEUROSCI.4248-08.2008.

Testa-Silva, G., Verhoog, M. B., Linaro, D., de Kock, C. P. J., Baayen, J. C., Meredith, R. M., et al. (2014). High Bandwidth Synaptic Communication and Frequency Tracking in Human Neocortex. PLoS Biol. 12. doi:10.1371/journal.pbio.1002007.

Trebbastoni, A., Pichiorri, F., D’Antonio, F., Campanelli, A., Onesti, E., Ceccanti, M., et al. (2016). Altered Cortical Synaptic Plasticity in Response to 5-Hz Repetitive Transcranial Magnetic Stimulation as a New Electrophysiological Finding in Amnestic Mild Cognitive Impairment Converting to Alzheimer's Disease: Results from a 4-year Prospective Cohort S. Front. Aging Neurosci. 7, 1-10. doi:10.3389/fnagi.2015.00253.

Turi, Z., Ambrus, G. G., Janacsek, K., Emmert, K., Hahn, L., Paulus, W., et al. (2013). Both the cutaneous sensation and phosphene perception are modulated in a frequencyspecific manner during transcranial alternating current stimulation. Restor. Neurol. Neurosci. 31, 275-85. doi:10.3233/RNN-120297.

Vargas-Leal, V., Bruno, R., Derfuss, T., Krumbholz, M., Hohlfeld, R., and Meinl, E. (2005). Expression and Function of Glial Cell Line-Derived Neurotrophic Factor Family Ligands and Their Receptors on Human Immune Cells. J. Immunol. 175, 2301-2308. doi:10.4049/jimmunol.175.4.2301.

Vlachos, A., Müller-Dahlhaus, F., Rosskopp, J., Lenz, M., Ziemann, U., and Deller, T. (2012). Repetitive Magnetic Stimulation Induces Functional and Structural Plasticity of Excitatory Postsynapses in Mouse Organotypic Hippocampal Slice Cultures. J. Neurosci. 32, 17514-17523. doi:10.1523/JNEUROSCI.0409-12.2012.

Volkmann, J., Albanese, A., Antonini, A., Chaudhuri, K. R., Clarke, C. E., De Bie, R. M. A., et al. (2013). Selecting deep brain stimulation or infusion therapies in advanced Parkinson's disease: An evidence-based review. J. Neurol. 260, 2701-2714. doi:10.1007/s00415-012-6798-6. 
Wake, H., Lee, P. R., and Fields, R. D. (2011). Control of local protein synthesis and initial events in myelination by action potentials. Science 333, 1647-51. doi:10.1126/science.1206998.

Wan, L., Xia, R., and Ding, W. (2010). Short-term low-frequency electrical stimulation enhanced remyelination of injured peripheral nerves by inducing the promyelination effect of brain-derived neurotrophic factor on Schwann cell polarization. J. Neurosci. Res. 88, 2578-87. doi:10.1002/jnr.22426.

Wang, S., Sdrulla, A. D., DiSibio, G., Bush, G., Nofziger, D., Hicks, C., et al. (1998). Notch receptor activation inhibits oligodendrocyte differentiation. Neuron 21, 63-75. doi:10.1016/S0896-6273(00)80515-2.

Wassermann, E. M. (1998). Risk and safety of repetitive transcranial magnetic stimulation: report and suggested guidelines from the International Workshop on the Safety of Repetitive Transcranial Magnetic Stimulation , June 5-7, 1996. Electroencephalogr. Clin. Neurophysiol. 108, 1-16. doi:10.1016/S0168-5597(97)00096-8.

Waxman, S. G. (1997). Axon-glia interactions: building a smart nerve fiber. Curr. Biol. 7 , R406-R410. doi:10.1016/S0960-9822(06)00203-X.

Weiner, H. (2004). Multiple sclerosis is an inflammatory t-cell-mediated autoimmune disease. Arch. Neurol. 61, 1613-1615. Available at: http://dx.doi.org/10.1001/archneur.61.10.1613.

Weiner, H. L. (2008). A shift from adaptive to innate immunity: A potential mechanism of disease progression in multiple sclerosis. J. Neurol. 255, 3-11. doi:10.1007/s00415008-1002-8.

Whittaker, E. T. (1910). A history of the theories of aether and electricity: from the age of Descartes to the close of the nineteenth century.

Wolf, S. D., Dittel, B. N., Hardardottir, F., and Janeway, C. A. (1996). Experimental autoimmune encephalomyelitis induction in genetically B cell-deficient mice. J. Exp. Med. 184, 2271-8. doi:10.1084/jem.184.6.2271.

Wolswijk, G. (1998). Chronic stage multiple sclerosis lesions contain a relatively quiescent population of oligodendrocyte precursor cells. J. Neurosci. 18, 601-9. Available at: http://www.ncbi.nlm.nih.gov/pubmed/9425002. 
Wrzos, C., Winkler, A., Metz, I., Kayser, D. M., Thal, D. R., Wegner, C., et al. (2014). Early loss of oligodendrocytes in human and experimental neuromyelitis optica lesions. Acta Neuropathol. doi:10.1007/s00401-013-1220-8.

Wu, L. M. N., Williams, A., Delaney, A., Sherman, D. L., and Brophy, P. J. (2012). Increasing internodal distance in myelinated nerves accelerates nerve conduction to a flat maximum. Curr. Biol. 22, 1957-1961. doi:10.1016/j.cub.2012.08.025.

Young, K. M., Psachoulia, K., Tripathi, R. B., Dunn, S. J., Cossell, L., Attwell, D., et al. (2013). Oligodendrocyte dynamics in the healthy adult CNS: Evidence for myelin remodeling. Neuron 77, 873-885. doi:10.1016/j.neuron.2013.01.006.

Zonta, B., Tait, S., Melrose, S., Anderson, H., Harroch, S., Higginson, J., et al. (2008). Glial and neuronal isoforms of Neurofascin have distinct roles in the assembly of nodes of Ranvier in the central nervous system. J. Cell Biol. 181, 1169-1177. doi:10.1083/jcb.200712154. 